University of Louisville

ThinkIR: The University of Louisville's Institutional Repository

8-2017

\title{
Transient absorption studies of CdSe nanocluster passivated with phenyldithiocarbamate ligands.
}

\author{
Yizhou Xie \\ University of Louisville
}

Follow this and additional works at: https://ir.library.louisville.edu/etd

Part of the Materials Chemistry Commons, and the Physical Chemistry Commons

\section{Recommended Citation}

Xie, Yizhou, "Transient absorption studies of CdSe nanocluster passivated with phenyldithiocarbamate ligands." (2017). Electronic Theses and Dissertations. Paper 2780.

https://doi.org/10.18297/etd/2780

This Doctoral Dissertation is brought to you for free and open access by ThinkIR: The University of Louisville's Institutional Repository. It has been accepted for inclusion in Electronic Theses and Dissertations by an authorized administrator of ThinkIR: The University of Louisville's Institutional Repository. This title appears here courtesy of the author, who has retained all other copyrights. For more information, please contact thinkir@louisville.edu. 
TRANSIENT ABSORPTION STUDIES OF CdSe NANOCLUSTER

\title{
PASSIVATED WITH PHENYLDITHIOCARBAMATE LIGANDS
}

\author{
By \\ Yizhou Xie \\ Master of Science in Chemistry, University of Louisville, 2012
}

\author{
A Dissertation \\ Submitted to the Faculty of the \\ College of Arts and Sciences of the University of Louisville \\ in Partial Fulfillment of the Requirements \\ for the Degree of \\ Doctor of Philosophy \\ in Chemistry \\ Department of Chemistry \\ University of Louisville \\ Louisville, Kentucky
}

August 2017 


\section{Copyright 2017 by Yizhou Xie}

All rights reserved 

TRANSIENT ABSORPTION STUDIES OF CdSe NANOCLUSTER PASSIVATED WITH PHENYLDITHIOCARBAMATE LIGANDS

\author{
By \\ Yizhou Xie \\ A Dissertation Approved on \\ June $29^{\text {th }}, 2017$ \\ by the following Dissertation Committee:
}

Dr. Jinjun Liu

Dissertation Director

Dr. Sachin Handa

Dr. Byron Freelon

Dr. Ying Li 


\section{ACKNOWLEDGEMENTS}

This Ph.D. dissertation was developed in the Department of Chemistry, University of Louisville. I would never have been able to finish it without the guidance and the help from the professors and the staff of the Department of Chemistry, and other researchers

outside the department. I am also grateful for the unconditional support from my family and friends.

Foremost, I would like to express my sincere acknowledgement to my advisor, Professor Jinjun Liu, for giving me the opportunity to work on this fascinating project, and creating an excellent atmosphere in which to conduct my research. Professor Liu is a brilliant researcher and an amazing advisor. He continuously provided guidance and insightful advice on my research and my writings. He also has been patient and supportive to me inside and outside the lab. His knowledge, scientific attitude, engineering skills and critical thinking have guided me throughout my graduate years and will set an example in my future career. I could not have asked for a better mentor for my Ph.D. studies.

I am also very grateful to Professor and former Department Chair Richard J. Wittbort, for allowing me to be his teaching assistant of the physical chemistry courses. His brilliant mind enlightened me, and all the students in his physical chemistry lectures.

I would like to thank all my Ph.D. committee members: Professor Ying Li, Professor Sachin Handa, and Professor Byron Freelon. They kindly provided advice and guidance, so that I could better organize the work present in this dissertation. 
My thanks also go to Professor Sergio B. Mendes in the Department of Physics, for his insightful questions and suggestions toward my project.

I would like to thank our collaborators: Professor Rajesh Sardar and Dr Meghan Teunis, in the Department of Chemistry and Chemical Biology of Indiana University Purdue University Indianapolis. Without their invaluable ideas and expert knowledge, our collaborating project would not be as strong as it is.

I would also like to express my genuine gratitude to Professor Francis P. Zamborini, for the help and encouragement on my graduate work. His fascinating graduate level classes helped me build my fundamental understandings on nanoparticles and analytical instruments.

I would like to thank my advisor of my master studies, Professor Eugene Mueller. His brilliant mind and rigorous scientific attitude pushed me to be a better researcher. I want to thank Professor Richard M. Higashi in the College of Medicine at the University of Kentucky, for his lectures on analytical chemistry, and his guidance on scientific research.

I like to thank our former postdoc Dr. Bill Pandit for teaching me to obtain a transient absorption spectrum and calibrate the laser system. I also want to thank my friend and labmate Abdelqader Jamhawi, who provide lots of useful discussions and arguments. I am also very grateful for the discussion and support from my friend Dr. Sanjay Singh.

I would like to express my sincerest thanks and appreciation to the staff of Chemistry Department: Sabrina Haug, Sherry Nalley, Aaron Howell, and Steve Riley. Steve's engineering skills helped us in many situations in the lab. My thanks also go to the office of the Conn Center for Renewable Energy Research: Vice Director Andrew Marsh, Administrative Associate Eunice De Lourdes Salazar, and Director Mahendra Sunkara. 
Many projects could not be possible without Director Sunkara's continuous support to the Ultrafast Laser Facility.

I also want to extend my sincere thanks to all the members of the Department of Chemistry, the Conn Center, and the Shumaker Research Building of University of Louisville.

Last but not least, I would like to give a special thanks to my parents, who unconditionally supported me through all the years of my life. 


\section{DEDICATION}

This Dissertation is dedicated to my parents,

Geng Xie, and Dongchuan Zhou 


\begin{abstract}
TRANSIENT ABSORPTION STUDIES OF CdSe NANOCLUSTER PASSIVATED WITH PHENYLDITHIOCARBAMATE LIGANDS
\end{abstract}

\author{
Yizhou Xie
}

June 29, 2017

Within the next century, the global population will keep increasing, while our main energy sources such as oil, coal and natural gas will become depleted. Therefore, finding alternative clean and sustainable energy sources requires immediate attention and extracts intense research interests.

Quantum Dots (QDs) and Semiconductor nanocluster (SCNC) research is one of the rapidly growing fields in renewable energy studies, driven by the promising fact that the properties of such materials can be modulated by changing their size, shape, and structures. Combination of semiconductor nanostructures and organic ligands provides even wider possibilities for design and development of effective and task-specific nanostructures. Understanding of, and eventually control of, energetics, interfacial interaction, and photoinduced processes in such highly heterogeneous structures is critical to inventions of novel materials for photovoltaic devices. 
In the newly established Ultrafast Laser Facility in the Conn Center of Renewable Energy Research, transient absorption pump-probe spectroscopy (TAPPS) has been employed to investigate the charge transfer dynamics in nanostructures with solar cell applications.

After a brief introduction of quantum dots and quantum-dot solar cells, and ultrafast laser systems including TAPPS detection schemes, our work on some novel nanoclusterligand systems will be presented. In this work, ultrasmall (1.6 nm in diameter) cadmium selenide nanoclusters with precise size and mass $\left(\mathrm{Cd}_{34} \mathrm{Se}_{34}\right)$ were passivated by phenyldithiocarbamate (PDTC) ligand monolayers. Because of the quantum confinement effect, the ultrasmall and well-controlled size of the CdSe nanocluster results a series of discrete and well-resolved electron and hole states in its valence and conduction bands, respectively. With the help of transient absorption spectroscopy, a quantitative spectroscopic transient absorption study of energetics and dynamics of these conjugates are presented.

Sub-picosecond electron transfer (ET) and hole transfer (HT) processes from the SCNC core to their organic passivating monolayer were observed in TAPPS when excited at a higher photon energy state than its optical bandgap. Based on results from various control experiments and computational fitting works, photoinduced processes in the SCNCligand conjugates have been well understood: Strong coupling between hole states and the ground electronic state of the passivating ligands delocalizes the hole orbitals and facilitates the HT process. In addition, ET from the conduction band of the nanoclusters to the excited states of the ligands creates interfacial charge transfer states with sub-picosecond lifetime. Charge transfer dynamics of CdSe SCNCs with varies of para-substituted derivatives of the PDTC ligand were also studied. 
The strong quantum confinement and "magical size distribution" of the ultrasmall SCNC core and the versatility of the passivating ligands give these heterogeneous nanostructures very different material functions from the bulk, which leads to unique applications: from quantum dots solar cells, photocatalysis, to biofluorescence sensors and imaging. The understanding of the charge transfer dynamics will provide useful information and guide a better design for the device in different scientific and industry fields. 


\section{TABLE OF CONTENTS}

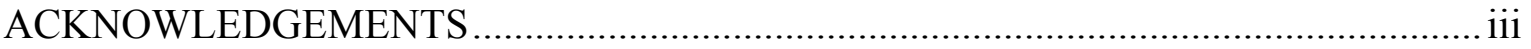

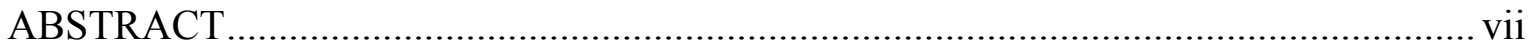

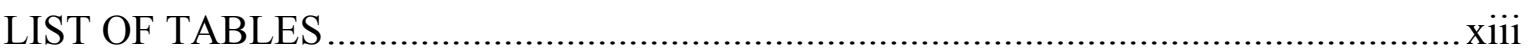

LIST OF FIGURES …………………………………...................................... xiv

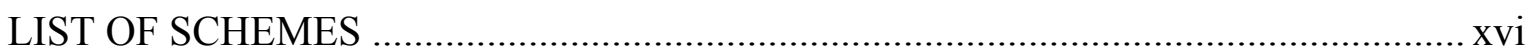

CHAPTER 1

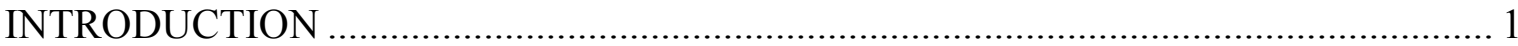

1.1 Global Energy Demands and Available Energy Resources ......................................... 1

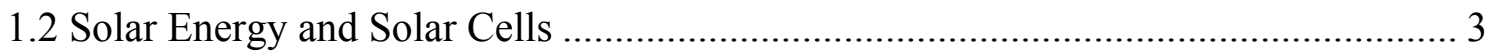

1.2.1 Electronic Band Structures of Semiconductor Materials ………………............... 4

1.2.2 Power Generation and Efficiency of a Solar Cell............................................. 7

1.2.3 Shocley-Queisser Limit .............................................................................. 9

1.3 Quantum Dots (QDs) and Quantum-Dot Solar Cells.............................................. 11

1.3.1 Quantum Confinement Effect............................................................................. 11

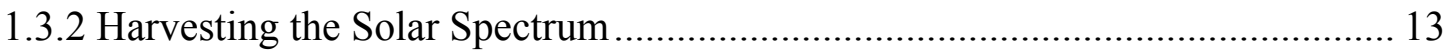

1.3.3 Atom-Like Energy Level Structures.................................................................. 15

1.3.4 Exciton Dynamics: Generation, Dissociation, and Recombination ................... 18

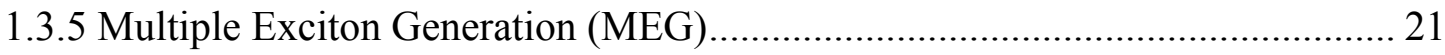

1.3.6 Photoinduced Charge Carrier Dynamics …………………………………...... 22

1.3.7 Advantages of Implementing Quantum Dots in Photovoltaic Devices.............. 25

1.4 Quantum Dots Synthesis and Surface Chemistry .................................................... 26

1.4.1 Ligand Exchange ……………………………………............................ 28

1.4.2 Core-Shell Quantum Dots .................................................................... 29

1.4.3 Deposition Methods...................................................................................... 31

1.5 Other Applications of Quantum Dots ................................................................ 32 


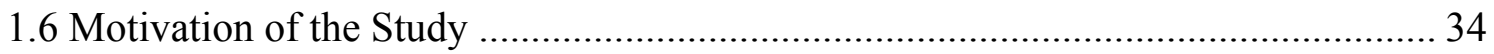

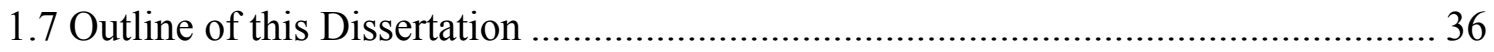

CHAPTER 2

ULTRAFAST TRANSIENT ABSORPTION LASER SPECTROSCOPY ......................... 38

2.1 Femtosecond Lasers and Amplifiers ……………………………………………..... 39

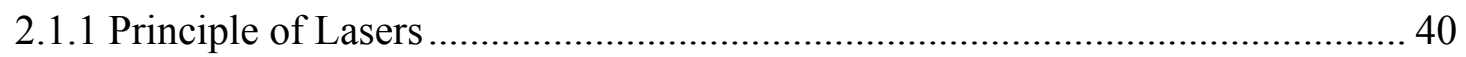

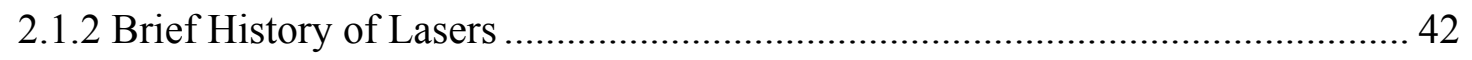

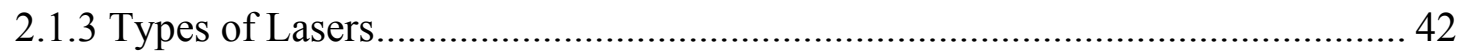

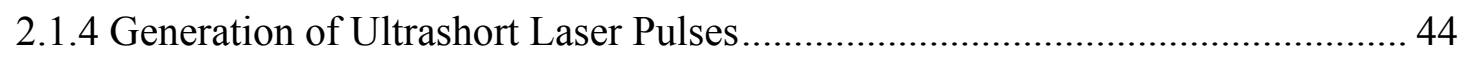

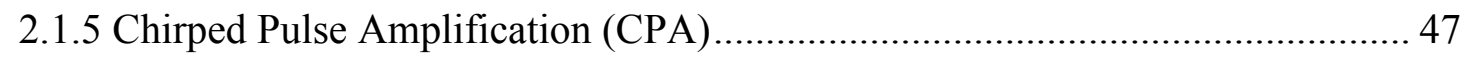

2.1.6 Femtosecond Lasers in the Present Work ....................................................... 48

2.2 Frequency Conversion Methods......................................................................... 52

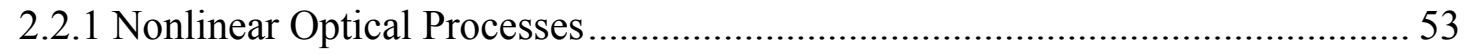

2.2.2 Phase Matching Conditions ........................................................................ 57

2.2.3 Supercontinuum Generation ............................................................................. 60

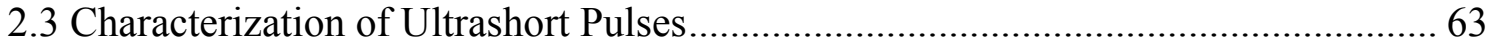

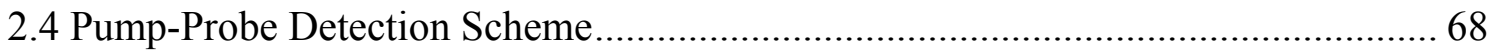

2.5 Experimental Setups of Femtosecond Laser System …………………………..... 72

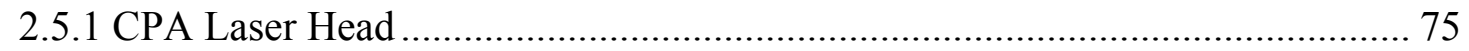

2.5.2 Pump Pulse Generation: NOPA and SHG...................................................... 77

2.5.3 Probe Pulse Generation: Supercontinuum White Light ..................................... 79

2.5.4 Transient Absorption Pump-Probe Spectroscopy (TAPPS) ................................ 83

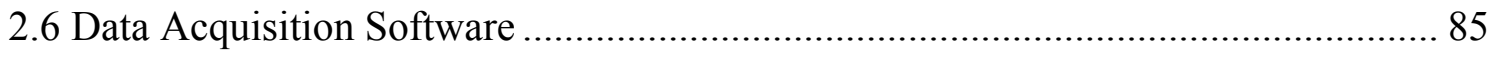

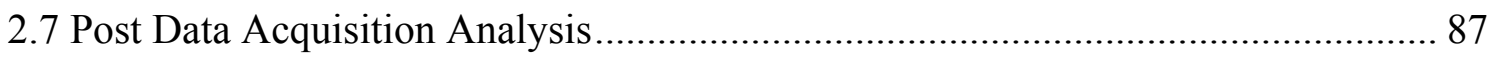

2.7.1 Group Velocity Dispersion (GVD) Correction …….......................................... 87

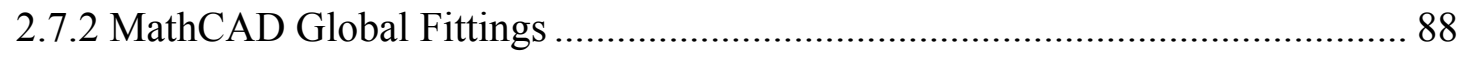

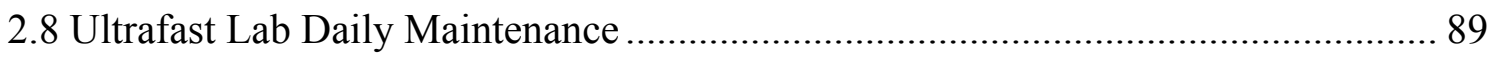

CHAPTER 3

ENERGY LEVEL ALIGNMENT AND PHOTOINDUCED ULTRAFAST CHARGE TRANSFER PROCESSES OF CdSe NANOCLUSTER PASSIVATED WITH LIGAND

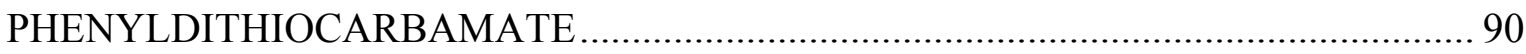




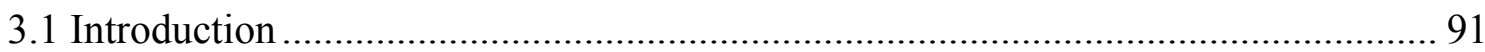

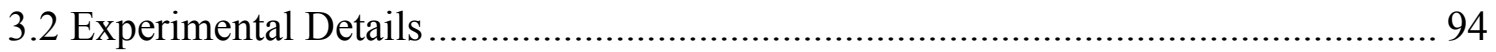

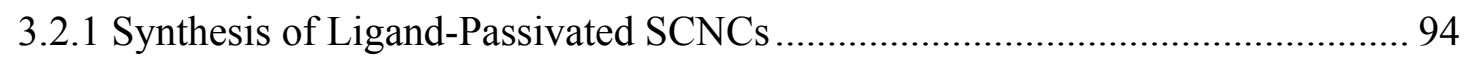

3.2.2 Transient Absorption Spectroscopy. ……………………………………….... 96

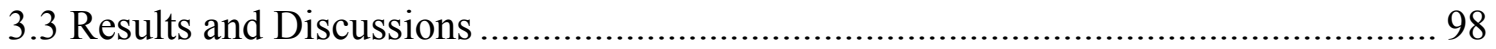

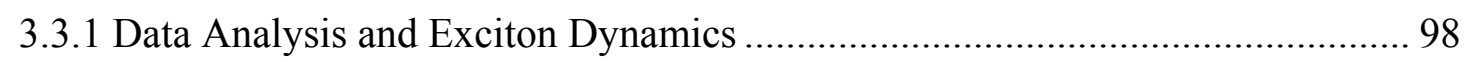

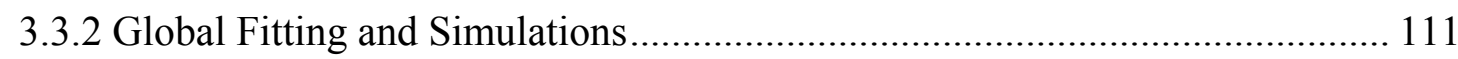

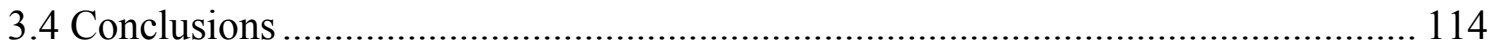

CHAPTER 4

CdSe NANOCLUSTERS PASSIVATED WITH PARA-SUBSTITUTED DERIVATIVES OF PHENYLDITHIOCARBAMATE LIGANDS ……………………....................... 118

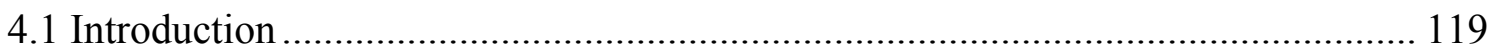

4.1.1 Electron Withdrawing/Donating Effect.......................................................... 124

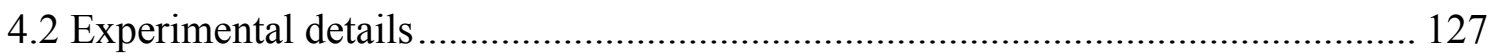

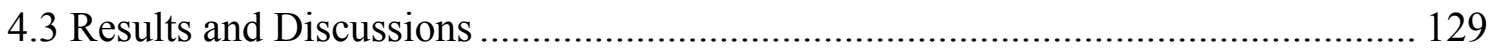

4.3.1 Wavelength Domain of the TA Spectra ………............................................... 129

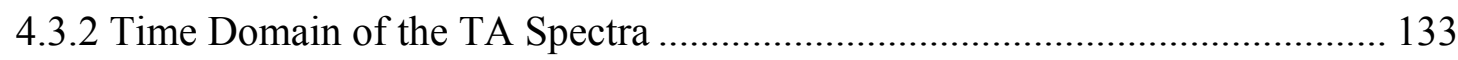

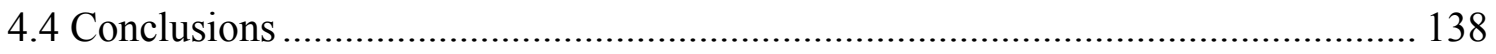

CHAPTER 5

GLOBAL CONCLUSIONS AND FUTURE DIRECTIONS ....................................... 139

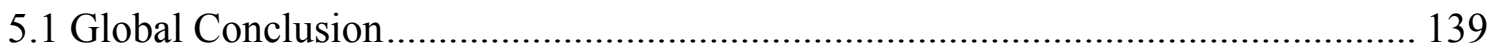

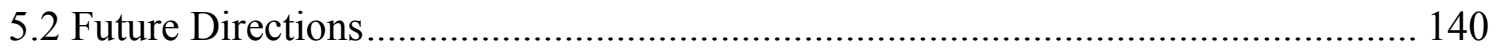

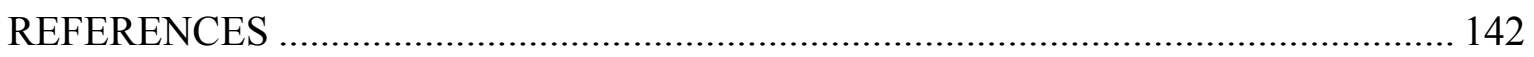

APPENDIX I COPYRIGHT PERMISSION ......................................................... 160

APPENDIX II MATHCAD GLOBAL FITTING ………………………………........ 163

APPENDIX III LIST OF ABBREVIATIONS …………………………………..... 169

APPENDIX IV GROUP VELOCITY DISPERSION CORRECTION ……………..... 172

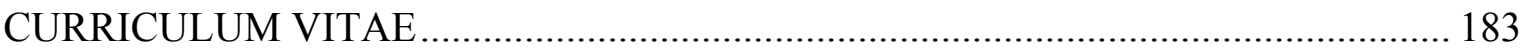




\section{LIST OF TABLES}

Table 2.1. Micrometer Readings of Delay Time and Crystal Angle in NOPA 1 and 2 ... 78

Table 3.1. Parameters Determined in the Global Fitting of the TA Spectra..................... 113

Table 4.1. Hammett Coefficients $(\sigma)$ and HOMO Energies of Function Groups ${ }^{\mathrm{a}}$.......... 123 


\section{LIST OF FIGURES}

Figure 1.1. Energy Band Structures in Solid Materials .................................................. 6

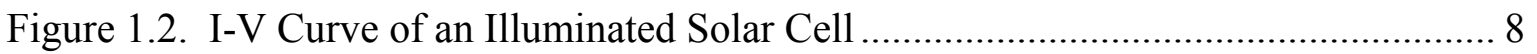

Figure 1.3. Contributions to Photon Energy Losses in a Solar Cell ................................ 10

Figure 1.4. Solar Radiation Spectra within the Atmosphere ......................................... 14

Figure 1.5. Schematic Illustrations of the Architecture of a Tandem QDs Solar Cell ..... 15

Figure 1.6. Electron Energy Levels and Size-dependent Band Gaps ............................... 16

Figure 1.7. Quantum Number-Denoted Energy Levels and Optical Interband Transitions

Figure 1.8. Exciton Recombination and Auger Effect................................................ 20

Figure 1.9. Schematic Diagram of Multiple Exciton Generation (MEG).......................... 22

Figure 1.10. Wave function (a) and Energy Level Alignments between the Holedelocalizing Phenyldithiocarbamate (PDTC) Ligands and the CdSe QDs......................... 24

Figure 1.11. Hot Injection Method to Synthesize Quantum Dots...................................... 27

Figure 1.12. Band Gap Offset of Type I (a) and Type II (b and c) Core/Shell Structures. 30

Figure 2.1. Absorption and Fluorescence Spectrum of Ti:Sapphire Crystal...................... 46

Figure 2.2. Schematic Diagram of the Components in CPA-Series Laser Head................ 49

Figure 2.3. Pulse Train of the Ti:Sapphire Regenerative Amplifier.................................. 52

Figure 2.4. Energy Conservation of Nonlinear Optical Processes...................................... 55

Figure 2.5. Type II (eo-e) Phase Matching (a) and Refractive Indices (b) in Uniaxial

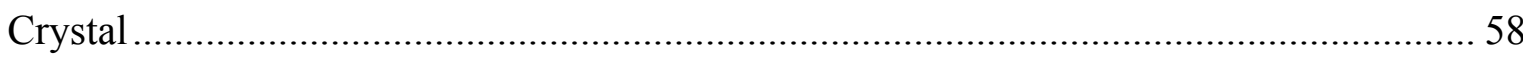

Figure 2.6. Schematic Diagram of Nonlinear Optical Parametric Amplification (NOPA) 60

Figure 2.7. Spectra of CPA Fundamental $(775 \mathrm{~nm})$ and NOPA Output $(532 \mathrm{~nm})$...............63 63

Figure 2.8. Schematic Diagram for Non-collinear Autocorrelation of $530 \mathrm{~nm}$................... 64

Figure 2.9. Intensity of Autocorrelation Signal Plotted Against Temporal Delay ............. 66

Figure 2.10. Second Harmonic Generation FROG Trace for a Negatively Chirped Pulse. 68

Figure 2.11. Pump-probe Detection Schemes with Two Different Probe Source .............. 70

Figure 2.12. Schematic Sketch of the Contributions of TA Signal .................................... 72

Figure 2.13. The Layout of the Femtosecond Laser Systems on the Optical Table .......... 74

Figure 2.14. Stanford Research System Lock-in Amplifier ............................................ 80

Figure 2.15. Supercontinuum White Light Generation and Detection .............................. 82

Figure 2.16. Monochromatic Porbe Source Detection Schemes ........................................ 84 
Figure 2.17. Group Velocity Dispersion (GVD) Correction of 3D Transient Absorption Spectra

Figure 3.1. Three-dimension Transient Absorption Spectrum of $1.6 \mathrm{~nm}$ CdSe

Semiconductor Nanoclusters Passivated with Phenyldithiocarbamate. 93

Figure 3.2. Steady-state absorption spectrum (top) and Transient absorption spectra at different pump-probe delay times (bottom) of PDTC-passivated CdSe SCNCs ( $\mathrm{d}=1.6$ $\mathrm{nm})$.

Figure 3.3 Time domain transient spectra of PDTC passivated $1.6 \mathrm{~nm} \mathrm{CdSe.}$ 102 Figure 3.4. Ultrafast kinetics of PDTC-passivated CdSe SCNCs at four wavelengths with electron quencher (Red trace) or hole quencher (Blue trace). 104

Figure 3.5. Steady-states absorption spectrum of PDTC in water. 105 Figure 3.6. Three dimensional TA spectrum of DDA-coated CdSe SCNCs with DCM as solvent and pumped at $388 \mathrm{~nm}$. 106 Figure 3.7. TA spectra of PDTC-passivated $1.6 \mathrm{~nm}$ CdSe SCNCs with $490 \mathrm{~nm}$ pump wavelength. (Inset shows decay kinetics at $545 \mathrm{~nm}$ )..................................................... 108 Figure 3.8. Steady States Absorption Spectra of $2.9 \mathrm{~nm}$ diameter CdSe QDs ................. 109 Figure 3.9. Transient Absorption (TA) Spectra of CdSe QDs-PDTC ligand conjugate.. 110 Figure 3.10. Simulation of PDTC-passivated $1.6 \mathrm{~nm}$ CdSe SCNCs with $388 \mathrm{~nm}$ pump wavelength using parameters determined in the global fit. 115

Figure 4.1. (a) HOMO Energy of X-PDTC Ligands against the Hammett Coefficient of the Functional Groups; (b) Change in Excitonic Radius against HOMO Energy................... 122 Figure 4.2. Schemetic Electron Densities of Methyl- and Trifluoromethyl- PDTCs ....... 125 Figure 4.3. Combining Effect of Induction and Conjugation Resonance Structures........ 126 Figure 4.4. Wavelength Domain of TA Spectra of X-PDTC CdSe NCs at $\Delta \mathrm{t}=0.5 \mathrm{ps} . . .130$ Figure 4.5. Excitonic Transition Energies Are Plotted Against (a) Band-edge Energy of the

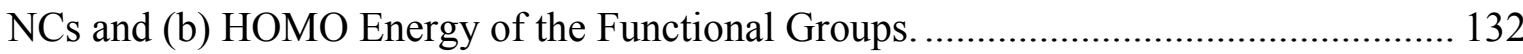
Figure 4.6. Transient Kinetics of X-PDTC-ligand-passivated CdSe NCs........................ 134 Figure 4.7. Transient Kinetics of X-PDTC-ligand-passivated CdSe NCs........................ 135 Figure 4.8. (a-e) Transient Kinetics of Hole Transfer form CdSe NCs to X-PDTC Ligands. (f) Time Constants of Hole Transfer plotted against the HOMO Energy. ....................... 136

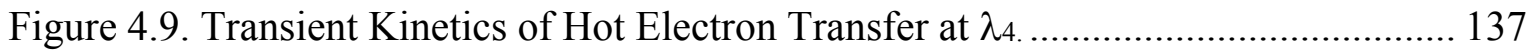




\section{LIST OF SCHEMES}

Scheme 3.1. Energy diagram and photoinduced processes in the CdSe SCNC-PDTC conjugate. 


\section{CHAPTER 1 \\ INTRODUCTION}

Research reported in this dissertation, namely ultrafast spectroscopic investigations of photo-induced charge carrier dynamics in ligand-passivated quantum dots (QDs) is motivated by the global demand for renewable energy sources and the urgent need to improve power conversion efficiency (PCE) of solar cells. Quantum dots are promising candidates for harvesting solar energy and may be utilized to surpass the PCE limit of photovoltaic devices. In this chapter, a brief description of the current status of solar cell research is presented first, followed by basic knowledge of QDs and their applications, especially in solar cells. We conclude this chapter with the outline of this dissertation.

\subsection{Global Energy Demands and Available Energy Resources}

According to the Population Projections Report released by the United Nations recently, the world population is highly unlikely to stop increasing within this century. In fact, there is an $80 \%$ probability that the world population will reach 9.6 billion in 2050 , and 12.3 billion in 2100 [1]. In 2005, the energy consumption by human activity had an estimated annual growth of $1.5 \%$. The global demand will reach 30 terawatts (TW) in the mid-century, and this number will be doubled at the end of the century [2]. 
With the industrial development and population growth worldwide in the past decade, the increasing energy demands require immediate attention. Economically developing countries with large populations like China, India, and Brazil, have already started making additional power plants every week to meet their fast-growing energy demand in the near future [3].

The majority of world's energy supply is currently generated from fossil fuels such as coal, crude oil, and natural gas. However, all these traditional sources of energy face limited supply, and burning fossil fuels can increase the risk of global warming and climate change. Current estimation of the global reserves of fossil fuels suggests that, at today's level of extraction, humans will run out of coal in 2127, crude oil in 2067, and natural gas in 2069 [4].

The fact that fossil fuel is not sustainable is not the only concern. Using fossil fuels as energy supply contains other severe hidden social and environmental costs. Air and water pollution, land degradation, and destruction of ecosystems, are all associated with coal mining, gas drilling, and oil drilling. Burning fossil fuels at power stations, industrial factories, or in everyday automobiles that run on gas, induces the danger of asthma and cancer, and has strong impacts on global warming and sea level rising [5]. With the growing environmental concerns associated with fossil fuels, there has been an increasing support among scientists for finding alternative renewable energy sources, such as wind and solar power. 


\subsection{Solar Energy and Solar Cells}

There is $9 \times 10^{22} \mathrm{~J}$ of energy that reach earth every day from the Sun, and the daily consumption by human society is only about $9 \times 10^{18} \mathrm{~J}[6]$, which means that if mankind could harvest solar energy efficiently, the global energy demands would be met without jeopardizing the ecosystems on earth.

Solar energy provides an environmentally-friendly method to generate power. Unlike the unsustainable fossil fuels, solar energy generation harvests power from the Sun, a source that is essentially infinite, and converts sunlight to electricity. In addition, solar panels are very modular, making them easy to install both for residential households and commercial uses.

The first photovoltaic solar cell was made by French scientist Edmond Becquerel at the age of 19 in 1839. In 1883, Charles Fritz built the first solid-state solar cell that was made of the semiconductor selenium and coated with a thin layer of gold. However, his solar cell only had about $1 \%$ sunlight to electricity conversion efficiency, while most modern-day solar cells have a conversion rate of $15-20 \%$. In 1887 , the photoelectric effect was observed by German physicist Heinrich Hertz, and later explained by Albert Einstein who received the Nobel Prize in 1921 for his contribution on the topic. Their contributions laid the groundwork for all modern-day solar cells [7].

On April $25^{\text {th }}, 1954$, Bell Labs announced the invention of the first practical silicon solar cell, demonstrating $6 \%$ efficiency [8]. Over the next few decades, not only has our understanding of photovoltaic cells gradually increased, but also semiconductors have become more readily available for cheaper prices, providing further incentive to advance 
solar cell technology. As of 2015, the most efficient and cost-effective commercial rooftop solar panel has an efficiency of $24 \%$ [9].

\subsubsection{Electronic Band Structures of Semiconductor Materials}

A solar cell is a photovoltaic device that converts the energy of sunlight directly to electricity. Conventional solar cells are made of semiconductor materials. A semiconductor is a solid substance that has electrical conductivity between that of an insulator and that of a conductor. The conductivity of a solid is due to the electron energy band structures of the material. Figure 1.1 shows the band structures of a conductor, an insulator, and different types of semiconductors.

In band structure theory, a hypothetical electron energy level, which is called the Femi level, is used in solid state physics in order to analyze the energy levels in a solid. At thermodynamic equilibrium, this fermi level would have a $50 \%$ probability of being occupied, which means any energy bands that are lower than the Fermi level would be completely filled with electrons, and any energy bands that are higher than the Fermi level would be unfilled with electrons. The valence band is the highest filled band, while the conduction band is the lowest unfilled band. The valence band and conduction band are the closest energy bands to the Fermi level.

In insulators and semiconductors, the energy difference between the top of the valence band and the bottom of the conduction is generally referred as the band gap, where no electron states can exist, therefore, also called the forbidden band. Within the valence band, electrons are used to form bonds to neighbor atoms, while within the conduction band, electrons can move freely and conduct electricity. Therefore, the position of the Fermi level 
related to the conduction band and the valence band is the crucial factor in determining electron conductivity of a solid material.

In a conductor, the conduction band and the valence band overlaps, and the Fermi level lies within the overlaps (Figure 1.1.a). In this case, the conduction band is filled with electrons which conduct electricity. In an insulator or a semiconductor, the Fermi level lies in the band gap between the conduction band and the valence band (Figure 1.1.b and c). Therefore, the conduction band is unfilled, i.e., no electron conductivity. However, in semiconductors, the band gaps are small enough for the Fermi level to be thermally populated with electrons or places for electrons (which called "holes"). When provided with energy in the form of a photons or thermal vibration, electrons in the valence band can leap across the band gap of the semiconductor. This leads semiconductor materials having electrical conductivities between those of conductor and those of insulators. Furthermore, the energy bands can be shifted by doping semiconductor materials with extra electrons or holes. N-type semiconductors have extra electrons, and therefore are negatively charged. Ptype semiconductors have extra holes, and are positively charged. The Fermi level of the ptype semiconductor lies near the valence band, and that of n-type semiconductor lies near the conduction band. At thermal equilibrium, as shown in Figure 1.1.d, electrons can move from the $\mathrm{p}$-type to the n-type, and holes can move from the n-type to the p-type. 

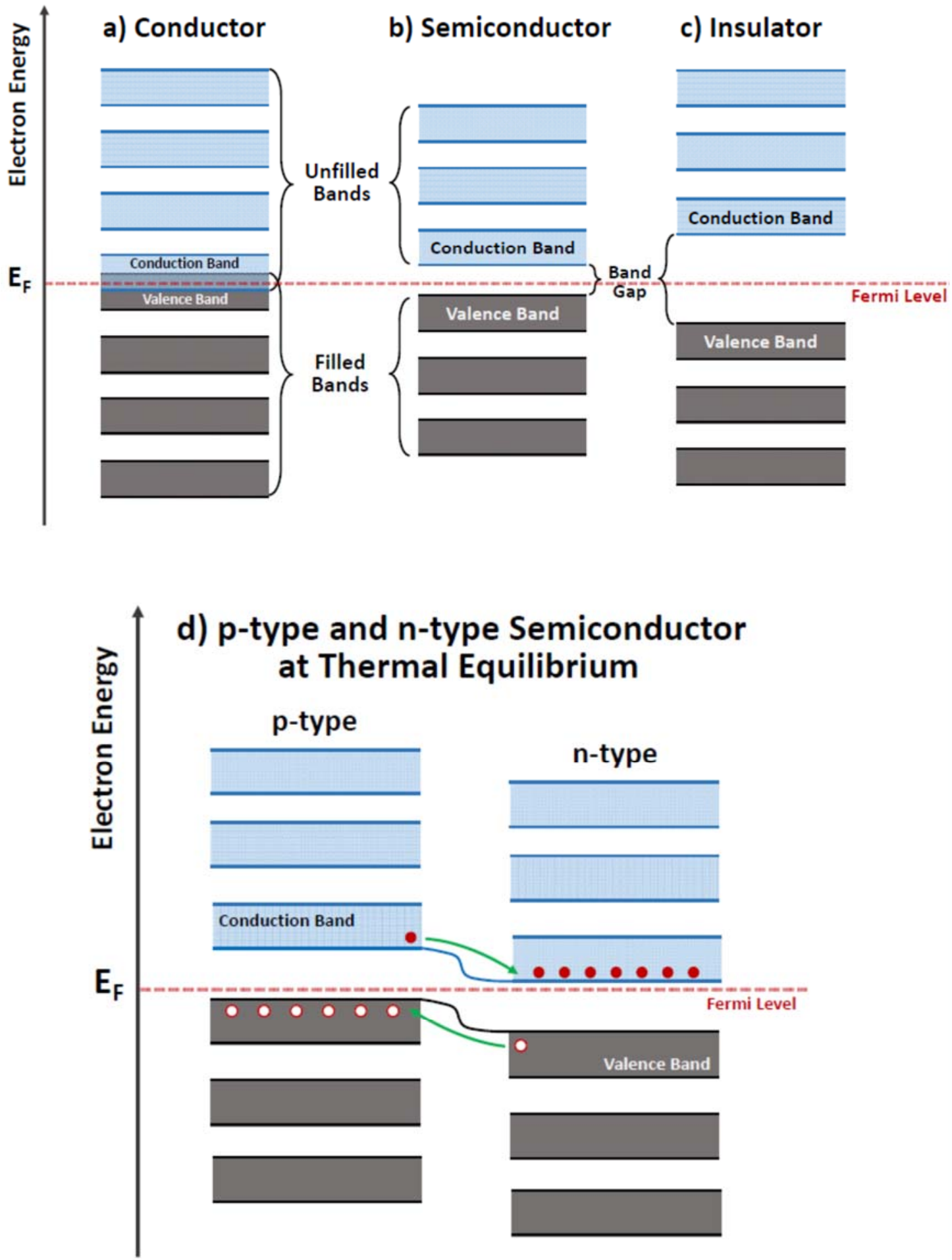

Figure 1.1. Energy Band Structures in Solid Materials 


\subsubsection{Power Generation and Efficiency of a Solar Cell}

A solar cell is made of a layer of n-type semiconductor and a layer of p-type semiconductor. Where the two layers of semiconductor meet, electrons can jump over the p-n junction, leaving positive charges on one side and negative charges on other side (Figure 1.3.a). The minimum energy required for an electron to leap from the valence band to the conduction band is called the band gap energy. If an incoming photon has energy greater or equal to the band gap energy of the semiconductor material, the photon will be absorbed by the $\mathrm{p}-\mathrm{n}$ junction, and the energy is transferred to an electron, generating an electron-hole pair. The electron and the hole can then be separated and drift towards opposite directions in the built-in electric field across the p-n junction and contribute to a net current $(I)$ within the solar cell device. With an external load, the current generates power $(P)$ which is equal to the current times the voltage $(V)$, or, $P=I \times V$.

The maximum amount of power that can be generated by a solar cell is limited by many factors, such as short-circuit current, open-circuit voltage, fill factor, and efficiency. The IV curve of a solar cell is shown in Figure 1.2. The short-circuit current $\left(I_{\mathbf{s c}}\right)$ is the current in the solar cell when the voltage across the cell is zero (i.e., the solar cell is short circuited). For an ideal solar cell with no resistive loss, the short-circuit current is equal to the maximum current that can be produced in the solar cell by photon excitation [10]. The open-circuit voltage $\left(V_{\mathbf{o c}}\right)$ is the maximum voltage available from a solar cell when the circuit is open and the current is zero [10]. $I_{\mathbf{s c}}$ and $V_{\mathbf{o c}}$ are the maximum current and voltage, respectively, from a solar cell. However, at both of these points, $\left(0, I_{\mathbf{s c}}\right)$ and $\left(V_{\mathbf{o c}}, 0\right)$, the power form the solar cell is zero. The maximum power $\left(P_{\mathbf{m a x}}\right)$ is generated at $\left(V_{\mathbf{m p}}, I_{\mathbf{m p}}\right)$, where the product of $V_{\mathbf{m p}}$ and $I_{\mathbf{m p}}$ equals to the largest rectangular area that will fit under the 
IV curve (Area A in Figure 1.2). The fill factor, commonly known as FF, is an important parameter that is used to measure the quality of the solar cell. It is defined as the ratio of the maximum power from the solar cell to the theoretical maximum power which is the product of $I_{\mathbf{s c}}$ and $V_{\mathbf{o c}}[11]$ :

$$
F F=\frac{P_{\max }}{P_{\text {theoretical }}}=\frac{I_{m p} V_{m p}}{I_{s c} V_{o c}}=\frac{\text { Area } A}{\text { Area } B}
$$

Graphically, FF is the ratio of the two rectangular areas in Figure 1.2. In real life application, a larger FF is desirable, and its corresponding IV curve is more square-like. A typical solar cell has a fill factor ranges from 0.5 to 0.82 .

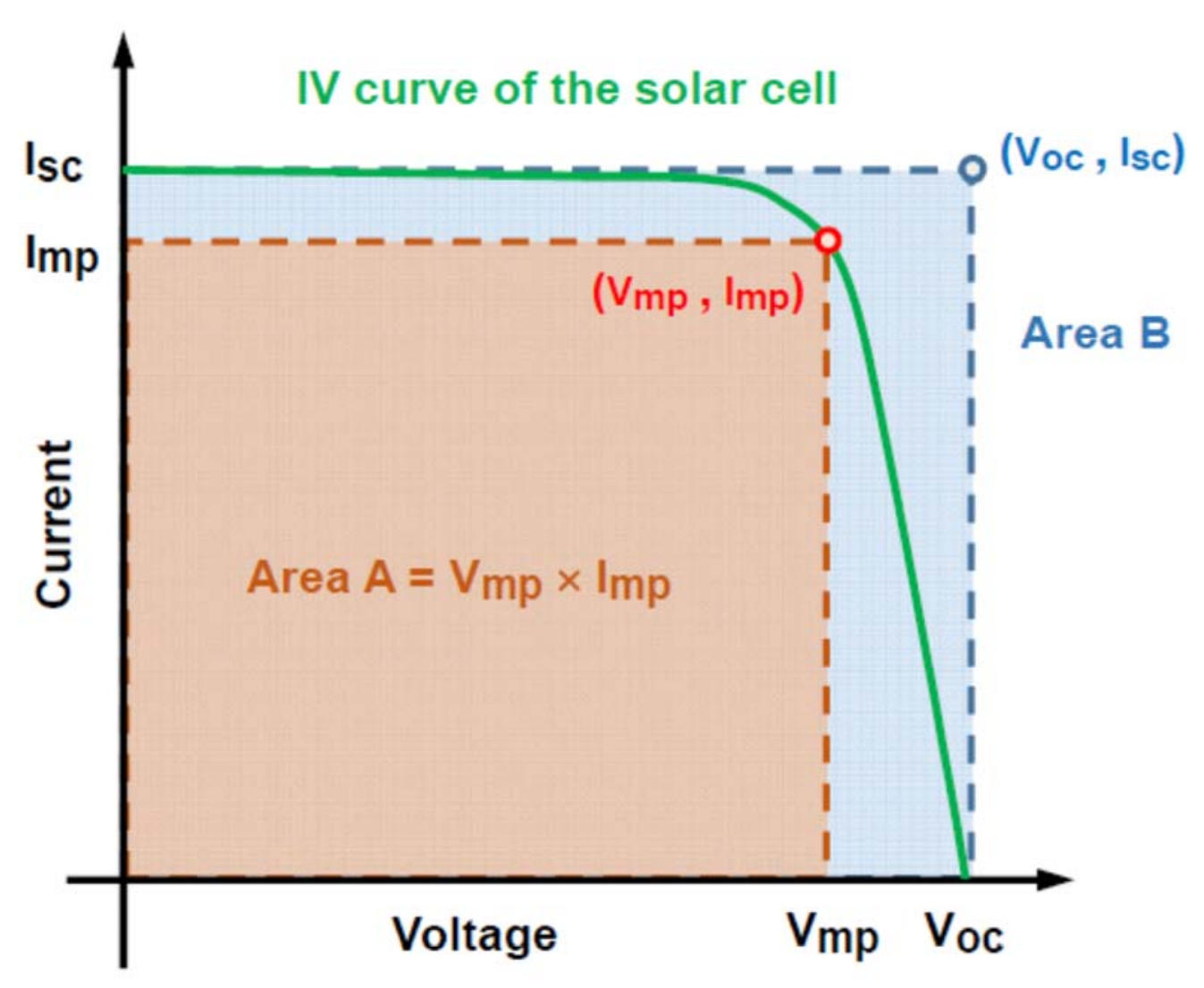

Figure 1.2. I-V Curve of an Illuminated Solar Cell 
Efficiency is another important parameter, which is the ratio of the electrical power output $\left(P_{\text {out }}\right)$ and the solar power input $\left(P_{\text {in }}\right)$. When the output power is equal to $P_{\max }$, the solar cell is operating at its maximum efficiency $\left(\eta_{\max }\right) . \eta_{\max }$ can be related to $I_{\mathbf{s c}}$ and $V_{\mathbf{o c}}$ using FF.

$$
\eta=\frac{P_{o u t}}{P_{\text {in }}} \Rightarrow \eta_{\max }=\frac{P_{\max }}{P_{\text {in }}}=\frac{I_{s c} V_{o c} F F}{P_{\text {in }}}
$$

These four quantities: $I_{\mathbf{s c}}, V_{\mathbf{o c}}, \mathrm{FF}$, and $\eta$ are the key parameters when characterizing the performance of a solar cell [10].

\subsubsection{Shocley-Queisser Limit}

In 1961, William Shockley and Hans Queisser [12] calculated the maximum theoretical efficiency of a single p-n junction silicon solar cell with a band gap energy of 1.1 $\mathrm{eV}$ (i.e. the band gap energy of the crystalline silicon). They concluded that, with all the sunlight hitting the solar panel, only $31 \%$ of the solar energy can be harvested and converted to electricity. This maximum conversion ratio is called the Shocley-Queisser Limit.

There are three main energy-loss mechanisms contributing to the Shockley-Queisser Limit. (1) Blackbody radiation. Any material that is not at absolute zero emits electromagnetic radiation, which is energy lost from the material (Figure 1.3.a). (2) Radiative recombination. An electron and a hole can recombine to emit a photon (Figure 1.3.b), which takes away energy. And (3) Spectrum losses. Incoming photons with energy less than the band gap (Ephoton $<\mathrm{Eg}$ ) cannot be absorbed by the junction and therefore "wasted". Photons with energy larger than the band gap ( $\left.E_{\text {photon }}>E_{g}\right)$ get absorbed, and the absorbed energy excites electrons to energy levels above the conduction band edge, from 
which the electrons quickly relax to the conduction band edge. The excess energy ( $E_{\text {photon }}-$ $\mathrm{E}_{\mathrm{g}}$ ) is converted to the vibration of the lattice and "wasted" (Figure 1.3.c).[12]

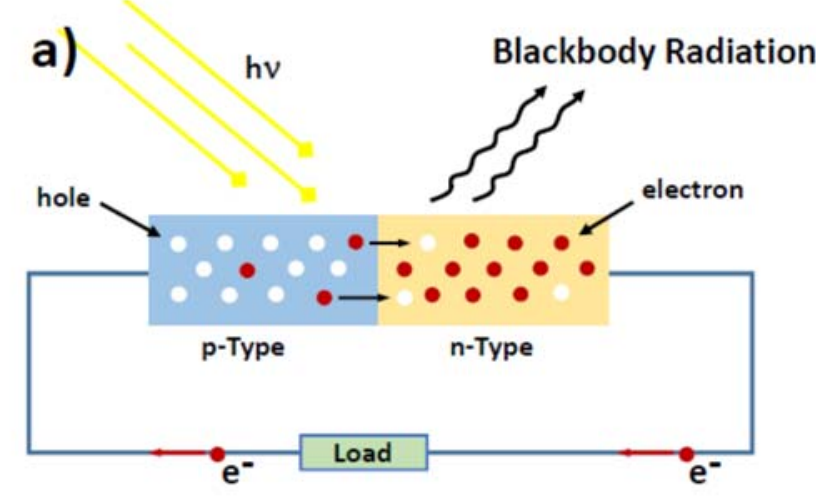

b)
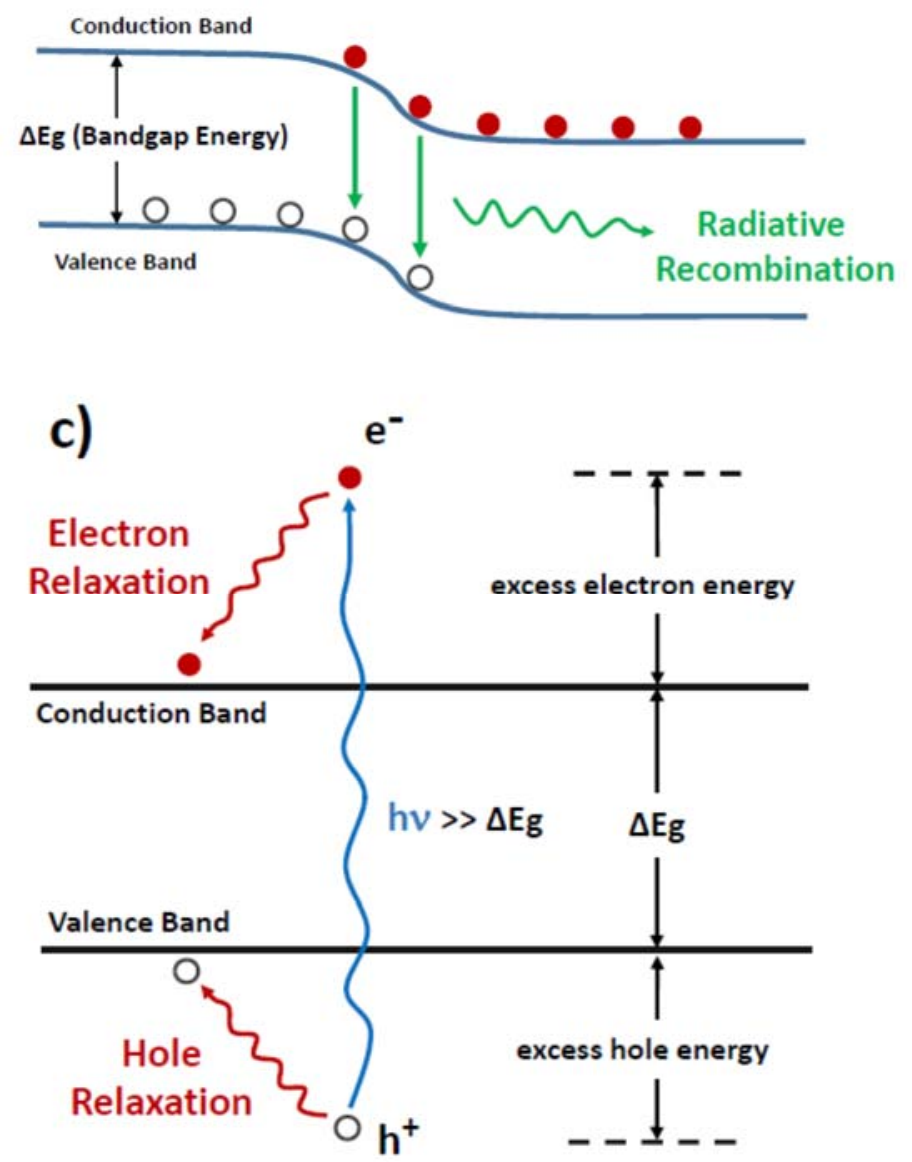

Figure 1.3. Contributions to Photon Energy Losses in a Solar Cell

There are three processes contributing to the photon energy losses. (a) Blackbody radiation, (b) Electron-hole radiative recombination, (c) Charge carrier relaxation. 


\subsection{Quantum Dots (QDs) and Quantum-Dot Solar Cells}

One key assumption that Shockley-Queisser Limit makes, is that only one electronhole pair can be created per incoming photon. Researchers have found that due to a multiple exciton generation (MEG) mechanism $[13,14]$ that exists in QDs material, QD solar cells theoretically can exceed the Shockley-Queisser Limit and reach much higher conversion efficiencies than bulk semiconductor solar cells $[15,16]$.

Quantum dots are small semiconductor nanocrystals that range in size from $2-10$ nm. Due to the extremely small size, the physics of these nanoparticles is governed by quantum mechanics. The size, band gap, and energy level structure of quantum dots can be precisely controlled, which makes them extremely useful in a variety of applications including implementations in solar cells.

\subsubsection{Quantum Confinement Effect}

One of the most interesting properties of quantum dots is that due to their diminutive size, the free charge carriers of QDs experience a phenomenon known as quantum confinement. Quantum confinement effect can be defined as the widening of the band gap as the size of the QDs decrease

Band gap energy of a semiconductor material is the energy required to create an electron and a hole when the electron rests at the minimum of the conduction band and the hole rests at the maximum of the valence band, i.e., the minimum energy separation between the conduction and the valence bands (usually in the unit of eV). As the dimensions of the material get smaller, the electron and the hole approach each other to a distance that the Coulombic interaction between them is no longer negligible, leading to a bound states of the 
two charged particles. The loosely bound electron-hole pair through Coulombic interaction is called an exciton.

Similar to the radius of the hydrogen atom in Bohr's model, the distance between the electron and the hole of an exciton, dubbed "the exciton Bohr radius", can be used to characterize the exciton. Let $m_{\mathbf{e}}$ and $m_{\mathbf{h}}$ be the effective masses of the electron and hole, respectively. (The mass of the hole $\left(m_{\mathbf{h}}\right)$ is much smaller than the mass of the proton $\left(m_{\mathbf{p}}\right)$ in Bohr's model). $\varepsilon$, $\hbar$, e are the optical dielectric constant, reduced Planck's constant, and the charge of an electron, respectively. The exciton Bohr radius can be expressed as Equation

\section{3.}

$$
r_{B}=\frac{\hbar^{2} \varepsilon}{e^{2}}\left(\frac{1}{m_{e}}+\frac{1}{m_{h}}\right)
$$

When the size of the quantum dots (diameter $=\mathrm{R}$ ) is equal to or smaller than the exciton Bohr radius $r_{\mathbf{B}}$, the electron and hole pairs are confined spatially to a dimension in which quantum mechanics starts to take over, (i.e., the quantum confinement effect occurs). Under quantum confinement, the behavior of an exciton in the quantum dot can be approximated using the classic "particle in a box" model. Therefore, rather than the standard energy continuum seen in bulk semiconductors, QDs have discrete or "quantized" energy levels.

The "particle in a box" model for quantum confinement in QDs was first proposed by Efros and Efros [17] in 1982 and later modified by Louis Brus [18] in 1983. An exciton in a quantum dot (with a diameter $=\mathrm{R}$ ) can be treated as a particle in a potential well with infinite potential barriers at its boundaries. The shift of band gap energy $\left(\Delta E_{\mathbf{g}}\right)$ due to quantum confinement effect is dictated by the Brus Equation: 


$$
\Delta E_{g}=E_{g}(\mathrm{QD})-E_{g}(\text { bulk })=\frac{\hbar^{2} \pi^{2}}{2 \mu R^{2}}\left(\frac{1}{m_{e}}+\frac{1}{m_{h}}\right)-\frac{1.78 e^{2}}{\varepsilon R}-0.248 E_{R y}^{*}
$$

The first term of this equation is the confinement energy of the exciton, similar to the zero-point-energy of a particle in an infinite square well potential. The second term represents the Columbic interaction between the electron and hole, called exciton binding energy. The last term is size independent, and called the Rydberg energy. The Rydberg energy, $E_{\mathbf{R y}}$, corresponds to the energy of the photon whose wavenumber is the Rydberg constant, i.e., the ionization energy of the hydrogen atom. In most cases, the last item is negligible. Overall, the bandgap of QDs is a function of the size. As the diameter (R) of a QD decreases, both the band gap energy and the separation between the intra-band energy levels increase. The band gap of QDs can be precisely tuned by changing their size [19-21].

\subsubsection{Harvesting the Solar Spectrum}

Figure 1.4 shows the solar radiation spectrum [22] of sunlight at the top of Earth's atmosphere. The wavelength distribution of the spectrum is similar to what would be expected from a $5250{ }^{\circ} \mathrm{C}(5523 \mathrm{~K})$ blackbody radiation. As sunlight passes through the atmosphere, some radiation energy is absorbed or scattered by gases in the atmosphere. The red trace in Figure 1.4 is the solar energy that reaches Earth's surface, and therefore can be harvested by photovoltaic devices.

A bulk semiconductor solar cell can have band-edge absorption of sunlight only at a certain wavelength, and the energy from the rest of the solar spectrum is wasted. Multijunction solar cells can have ban-edge absorption at different wavelength but are in general very expensive and highly unpractical for commercial productions. 


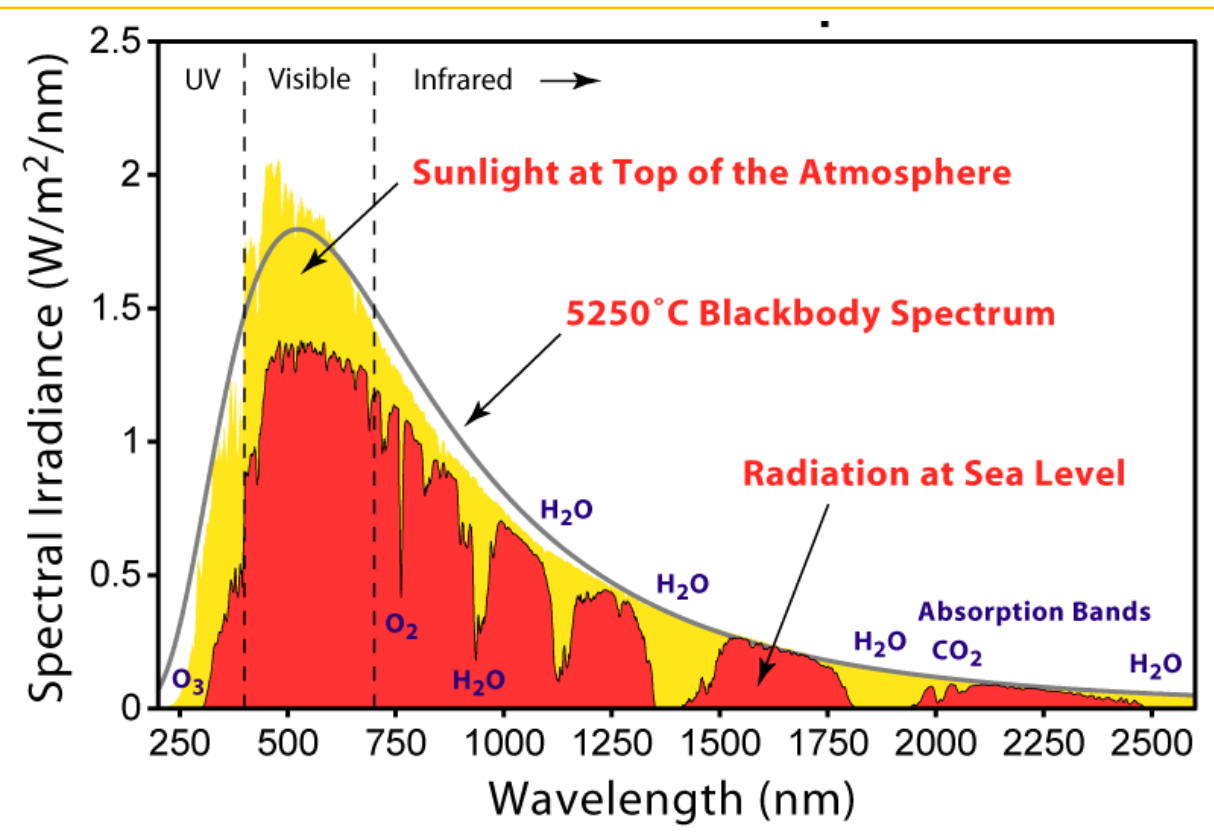

Figure 1.4. Solar Radiation Spectra within the Atmosphere

Figure was prepared by Robert A. Rohde for the Global Warming Art project. Permission for adaptation is granted under the terms of GNU Free Documentation.

On the other hand, QD solar cells can absorb sunlight at band-edge over a broad range of frequency due to the fact that the band gaps of the QDs can be precisely tuned over a broad range of the solar spectrum due to the quantum confinement effect. QDs with different dimensions and hence different band gaps can be mixed together and used as light absorbers. Through electrophoretic deposition with sequentially-layered architecture, tandem QD solar cells have been built to capture the incident visible and infrared photons [23]. The sequential layering of the large-band-gap CdSeS QDs followed by the small-bandgap QDs (Figure 1.5) maximized the light-harvesting capability across the solar spectrum. Therefore, QD solar cells are much more efficient than bulk semiconductor solar cells in harvesting solar energy, and could be a much cheaper alternative for the traditional multijunction methods $[20,21,24,25]$. 


\section{a) Schematic Structure of a Tandem QD solar cell with Sequentially deposited QDs}

Colloidal QDs

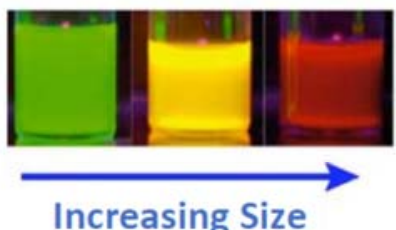

Increasing Size

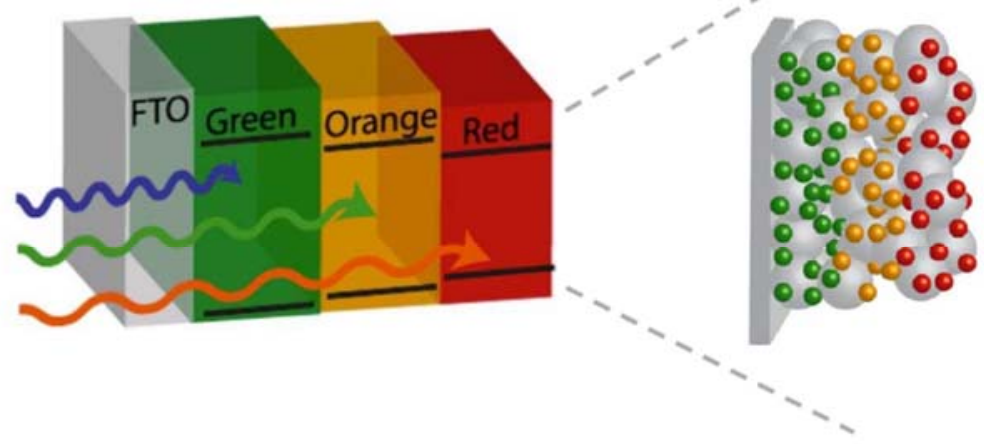

b) Illustration of Electron Transfer and Energy Transfer in the Tandem Architecture
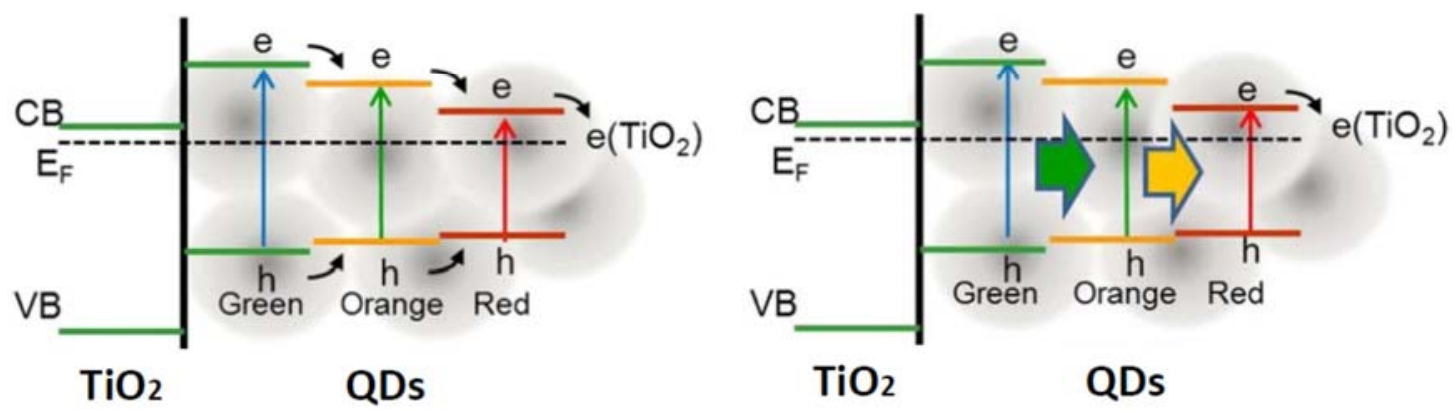

Figure 1.5. Schematic Illustrations of the Architecture of a Tandem QDs Solar Cell CB: Conduction band, VB: Valence band, $\mathrm{E}_{\mathrm{F}}$ : Fermi level. Figure adapted with permission from reference 23, Copyright 2013, American Chemistry Society.

\subsubsection{Atom-Like Energy Level Structures}

QDs $(d=2-10 \mathrm{~nm})$ and semiconductor nanoclusters $(d<2 \mathrm{~nm})$ bridge the gap between bulk materials and single molecules. In this size regime, quantum confinement effects lead to an observable increase of the bandgap. The discrete, quantized energy levels in the QDs result electronic structures that are intermediate between bulk semiconductors, which have continuous energy levels within the energy bands, and individual molecule, which have a single HOMO-LUMO gap (Figure 1.6).[26] 


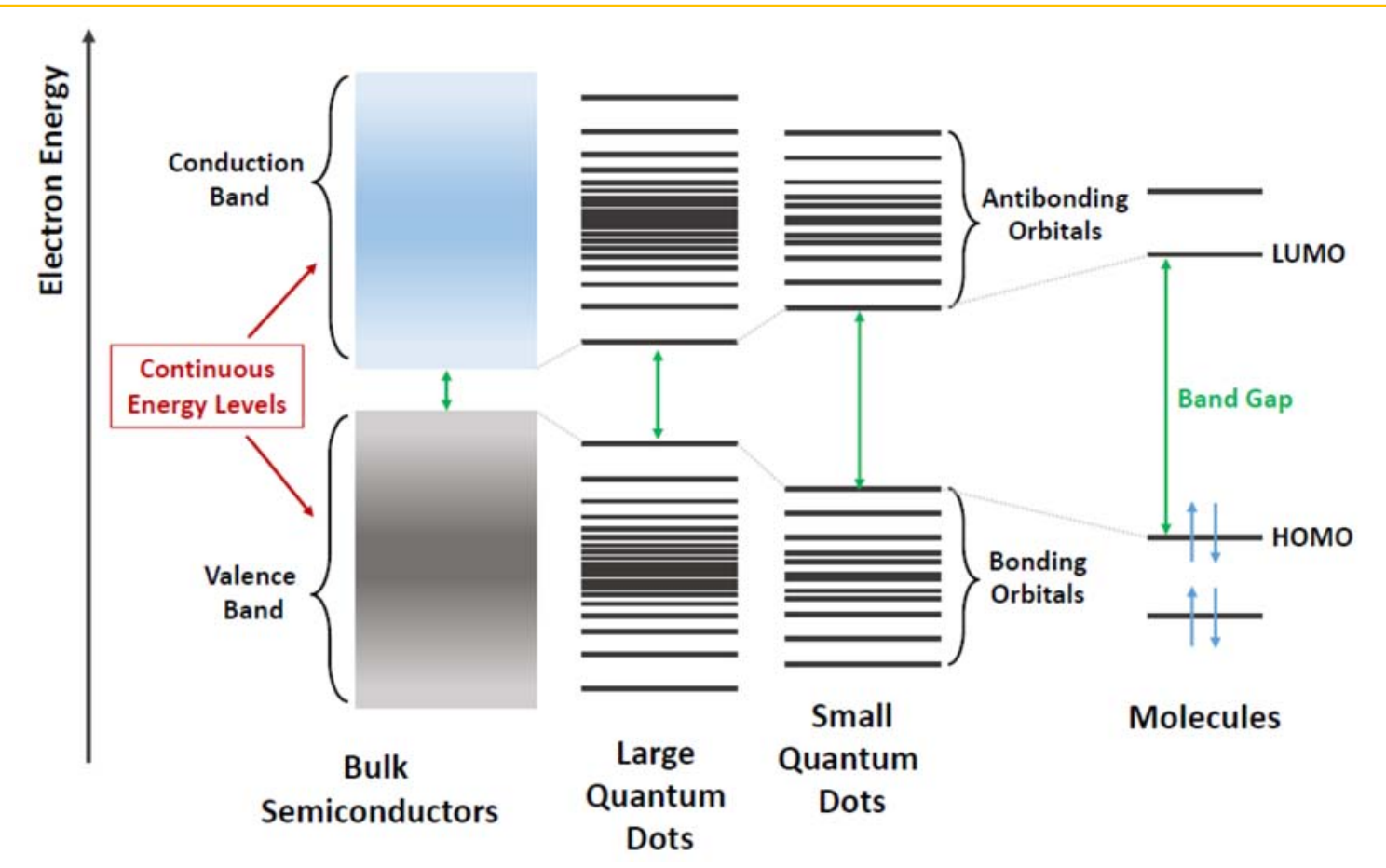

Figure 1.6. Electron Energy Levels and Size-dependent Band Gaps

As the size of the QDs gets smaller, the number of atoms decreases, and so does the number of the quantized energy levels. In the absence of the band-mixing effect, the discrete energy levels replace the continuous energy bands that are in bulk semiconductors, and each bulk bands gives rise to an independent series of quantized states, which can be classified using two quantum numbers: $L$ and $\boldsymbol{n}$ [27]. The angular momentum, $L$, determines the symmetry of the electron wave function, i.e., the shape of the orbital [27]. And the principal quantum number, $\boldsymbol{n}$, denotes the size of the orbital. In the typical notation of QDs quantized states, the momentum is indicated by a letter, ( $\mathrm{S}$ for $L=0, \mathrm{P}$ for $L=1, \mathrm{D}$ for $L=2$, and so on), and preceded by the value of $\boldsymbol{n}$. The three lowest energy states in the order of increasing energy are 1S, 1P and 1D (Figure 1.7.b). 


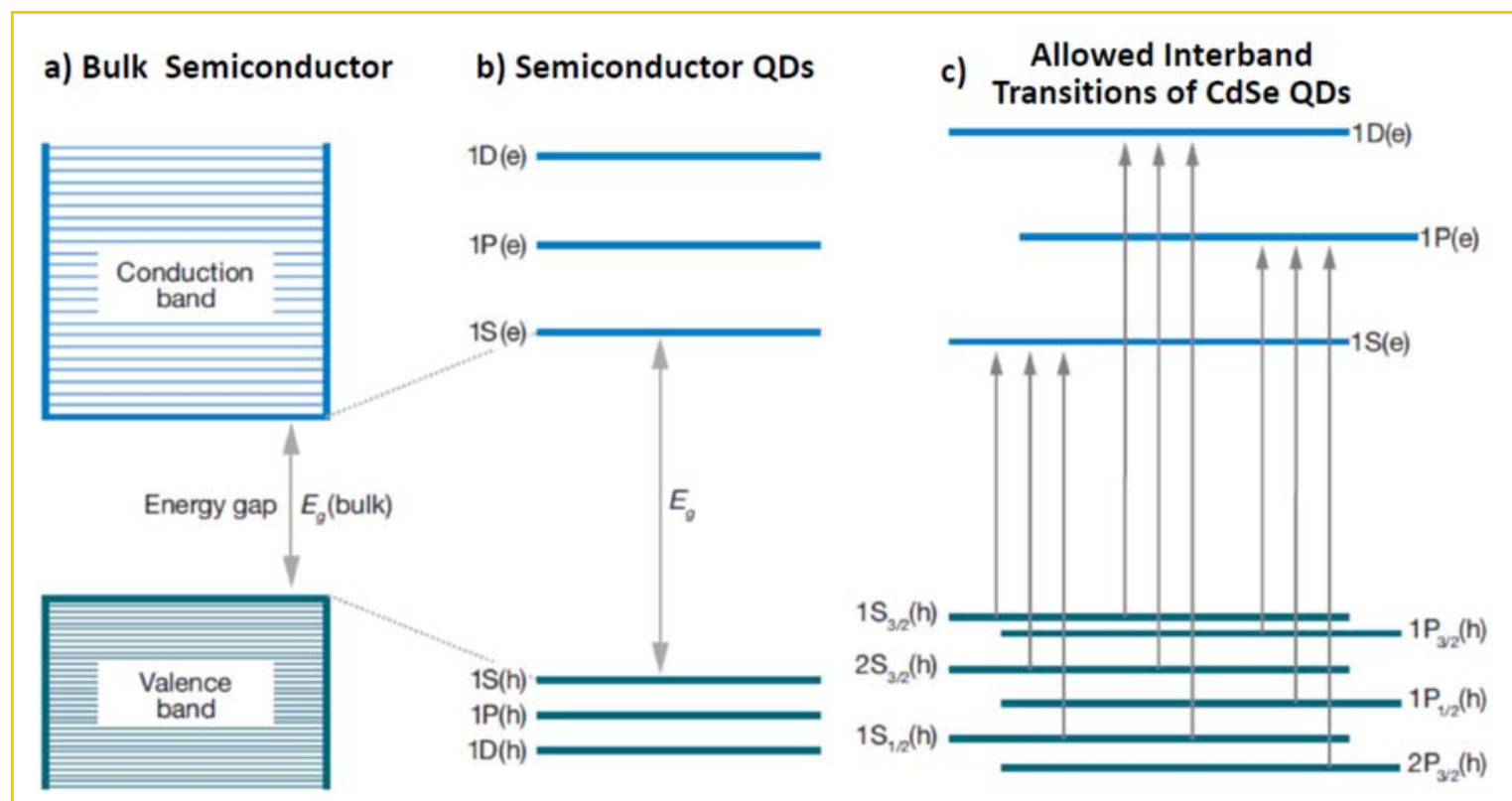

Figure 1.7. Quantum Number-Denoted Energy Levels and Optical Interband Transitions

Figure adapted with permission from reference 27. Copyright 2007, Annual Reviews.

However, while this notation provides a reasonable description of the electron states of a $\mathrm{QD}$, as $1 \mathrm{~S}(\mathrm{e}), 1 \mathrm{P}(\mathrm{e})$, and $1 \mathrm{D}(\mathrm{e})$, it oversimplifies in the case of the complex multisubband character of the hole states in typical semiconductor QDs [27]. Due to the quantum confinement-induced mixing between different valence subbands [28, 29], which produces a more complex structure of hole quantized states, the true quantum number of QDs hole states is the total angular momentum, $F$, and the hole states are usually denoted as $n L_{F}(\mathrm{~h})$. According to the hole energies calculations in CdSe QDs [28], the three lowest hole states are $1 \mathrm{~S}_{3 / 2}(\mathrm{~h}), 1 \mathrm{P}_{3 / 2}(\mathrm{~h})$, and $2 \mathrm{~S}_{3 / 2}(\mathrm{~h})$ (Figure 1.7.c). Optical transitions that involve these states are fully resolved in linear absorption spectra of colloidal CdSe QDs, and only several interband transitions are allowed, which are indicated by up arrows in Figure 1.7.c [27]. 
The theoretically predicted energies of those interband optical transitions in CdSe QDs were reported [30], and later confirmed by experiments [31].

\subsubsection{Exciton Dynamics: Generation, Dissociation, and Recombination}

A number of processes effect the PCE of QD solar cells: exciton generation (i.e., light absorption), exciton dissociation, exciton recombination, charge carrier transportation and recombination [32]. The first PCE limiting process is exciton generation. When sunlight hits the active film of solar devices, if the photon energy of the incident light is larger than the band gap of the semiconductor material, the solar irradiance can be absorbed to excite an electron from the valence band into the conduction band. The excited electron quickly falls back to the bottom of the conduction band through intraband relaxation. When an electron in the conduction band and a hole in the valence band are bounded through Coulombic interaction, a photoinduced exciton is generated. The bound state of the electron and hole pair need to be split into free charges in order to contribute to the photoconductivity, which is the second PCE limiting process: exciton dissociation. In order for a successful exciton dissociation, the photoinduced free charge carriers must be transported to inorganic ligands, or the shell structure of the nanocrystal, or an organic acceptor [33-35]. The distance that the charge carrier travels between exciton generation and exciton recombination is the diffusion length. Increasing the diffusion length, and reducing the rate of the recombination is essential for improving the PCE of solar devices [36, 37]. The three most common recombination are discussed here: Shockley-Read-Hall recombination, radiative recombination, and auger recombination. 
A. Shockley-Read-Hall Recombination (aka, Trap-assisted Recombination)

Shockley-Read-Hall (SRH) recombination process is named after three scientists: William Shockley, William Thornton Read [38] and Robert N. Hall [39]. First, an electron in the conduction band falls into a new localized energy state which is created within the band gap due to an impurity in the lattice. Then the electron in the trap state recombines with a hole in the valence band (Figure 1.8.a). Such energy states within the band gap are called deep-level traps, hence the name trap-assisted recombination.

\section{B. Radiative Recombination}

Radiative recombination is a form of spontaneous emission. During this process, a photon is emitted with the wavelength responding to the energy released from the electronhole recombination (Figure 1.8.b). Spontaneous emission often induces stimulated emission. When photons are present in the semiconductor material, they can either be absorbed and generate an exciton, or stimulate a radiative recombination, resulting in a new generated photon with similar properties to the original photon. Photon absorption is the key process in solar cells, while the latter, stimulated emission, is the basis of solid-state lasers and LEDs.[29]

\section{Auger Recombination.}

In Auger recombination, the energy is used to excite a third charge carrier (usually an electron) to a higher energy level without moving to another energy band (Figure 1.8.c). After this intraband excitation, the third carrier usually losses its excess energy to thermal vibrations. Auger recombination is a three-particle interaction, much like the Auger electron emission, in which the filling of an inner-shell vacancy of an atom is achieved by the 
emission of an electron from the same atom (Figure 1.8.d). These Auger effect processes are hard to produce, due to the fact that the third particle would have to be in an unstable high-energy state at the beginning of the process. $[13,40]$
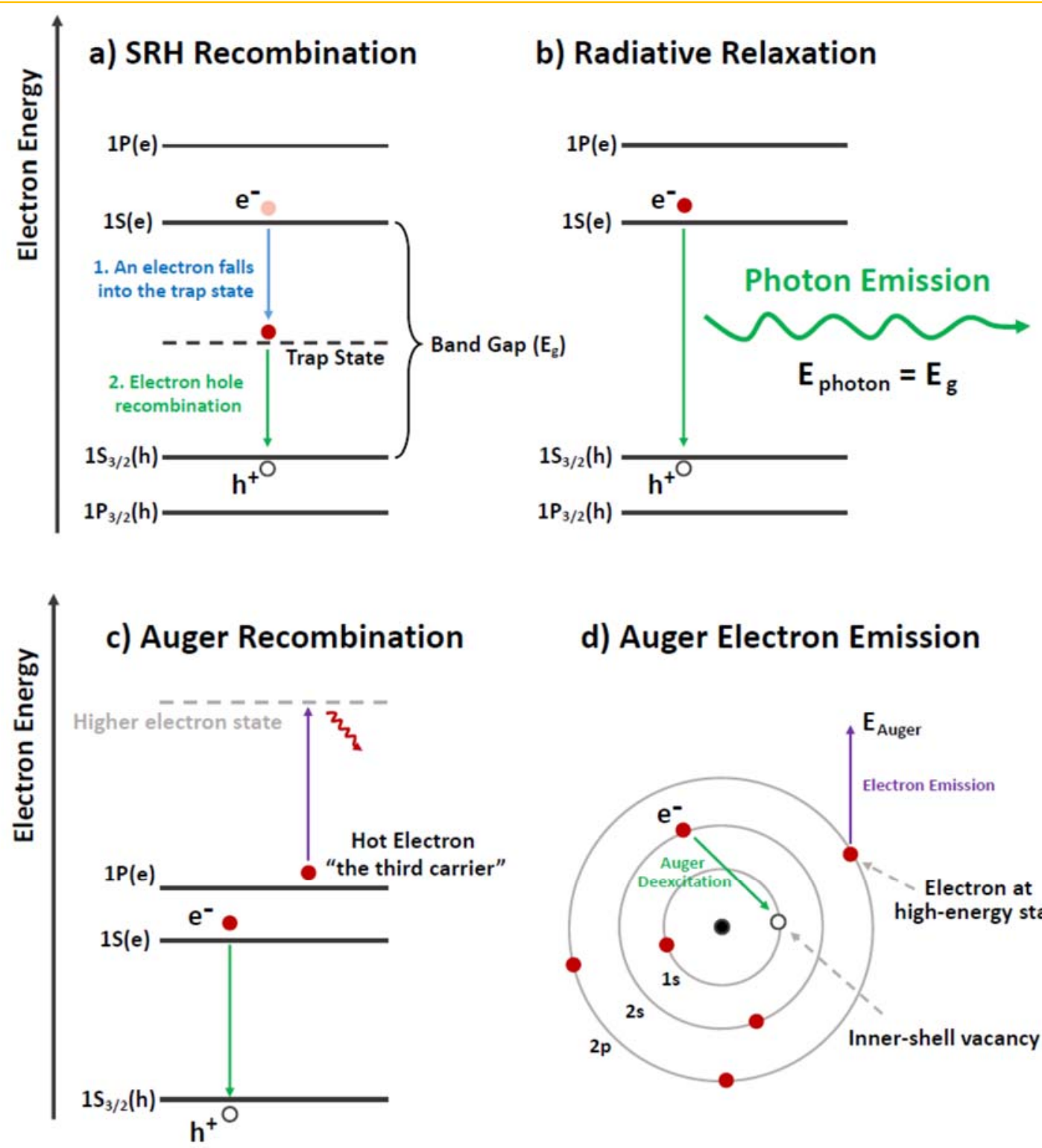

\section{d) Auger Electron Emission}

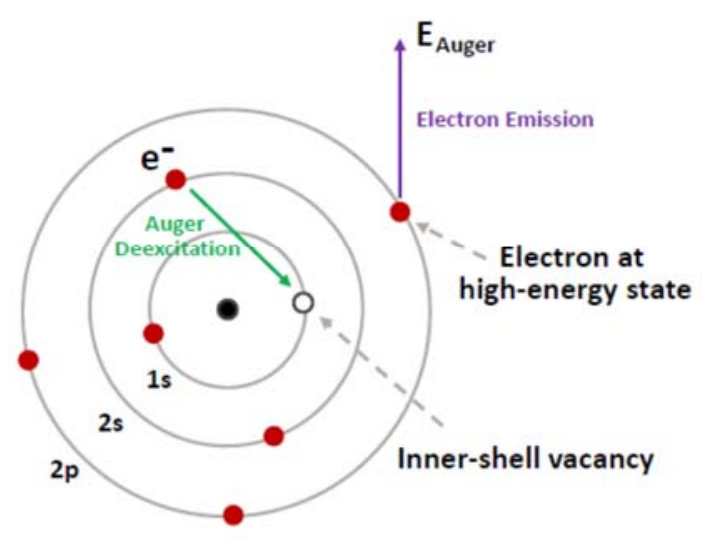

Figure 1.8. Exciton Recombination and Auger Effect 


\subsubsection{Multiple Exciton Generation (MEG)}

It has been experimentally observed [13, 14, 29, 41, 42] that within QDs, one incoming photon can generate multiple electron-hole pairs, which increases the quantum yield, which is defined as the number of electron-hole pairs created per photon. Since the bound state of an electron-hole pair is called an exciton, this phenomenon is called multiple exciton generation (MEG). MEG theoretically makes the efficiency of QD solar cells possible to exceed the Shockley-Queisser Limit.

In terms of energy conversion, QD solar cells function in the same way as traditional solar cells. Photons with energy higher than the band gap are absorbed, which creates electron-hole pairs. However, QD solar cells have an enhanced ability of harvesting solar energy. When the energy of a photon (hv) is greater than the band gap energy by at least two times, the photon energy is absorbed by the QD and an electron is excited to an energy level much higher than the conduction band edge (blue arrow in Figure 1.9). This electron quickly relaxes to the bottom of the conduction band by releasing energy. Unlike in bulk materials, the released energy does not transfer to lattice vibration (i.e. generating a phonon), but transfers to another electron through impact ionization, which excites the second electron to the conduction band (green up arrow in Figure 1.9). The whole process produces two electron-hole pairs from one photon, and multiple electron-hole pairs may be produced with sufficient photon energy. Conventionally, researchers have referred MEG as a non-radiative reverse effect of the Auger recombination.

MEG is not exclusive to QDs, but could occurs in traditional solar cells as well. However, in bulk semiconductors, the rate of impact ionization is much slower than that of phonon emission. So most of the excessive photon energy is lost via phonon-electron 
scattering. Only when the photon energy is many times of the band gap energy, the rate of the impact ionization becomes competitive to the phonon emission, and MEG becomes relevant. As a result, the threshold photon energy for bulk semiconductors to have a quantum yield greater than $100 \%$ is in the deep ultraviolet, which is out of the solar spectrum on earth surface. Thus, MEG is much more rare in bulk material solar cells than in QD solar cells.

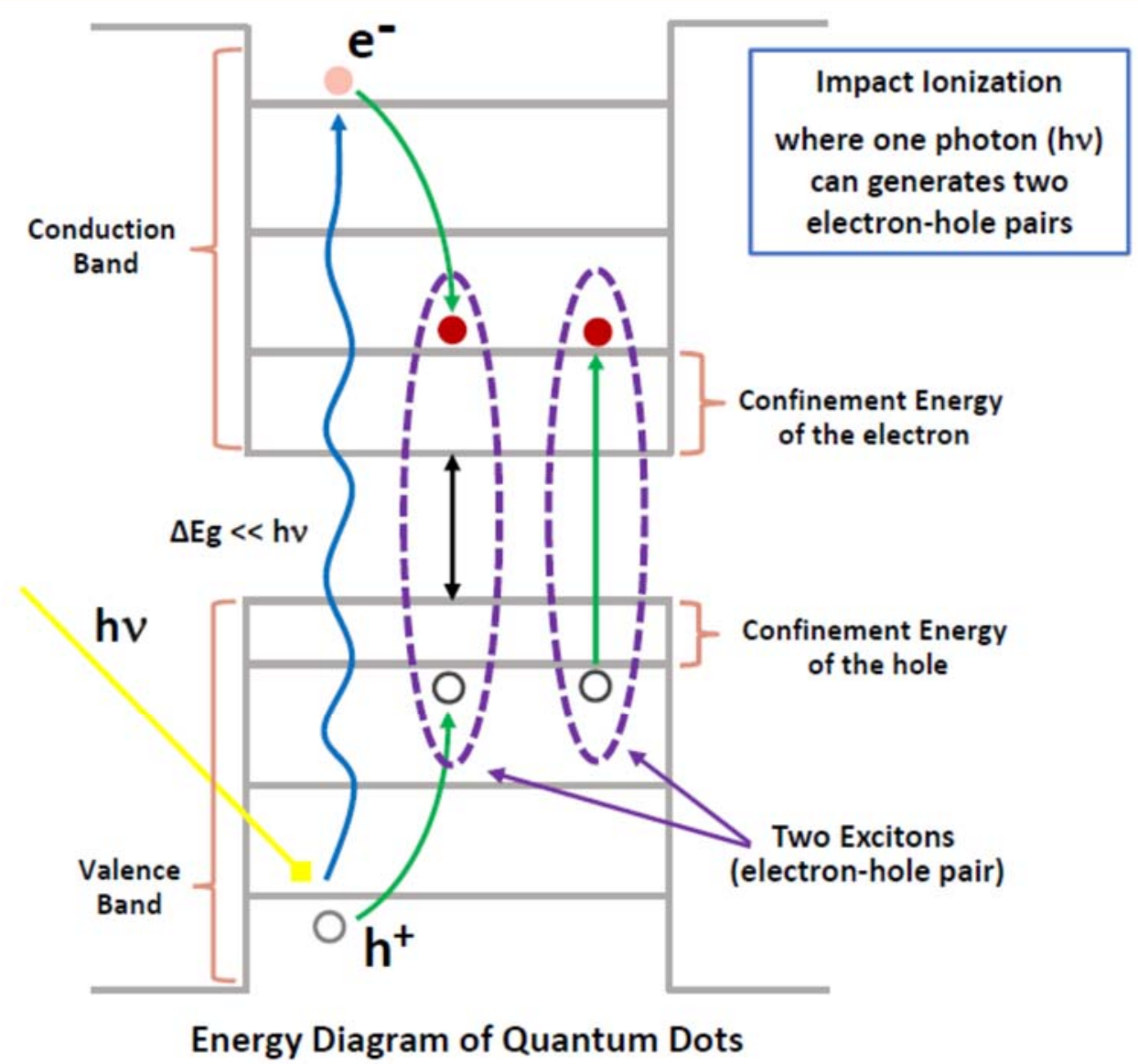

Figure 1.9. Schematic Diagram of Multiple Exciton Generation (MEG)

\subsubsection{Photoinduced Charge Carrier Dynamics}

In QD solar cells, various semiconductor nanostructures (zero-dimension QDs, onedimension nanorods, two-dimension nanosheets, core/shell QDs, and ligand-passivated QDs) have been widely investigated as the light absorber and charge transfer components, 
due to their novel properties of tunable band gap, large cross-section, and fast charge separation. It is important to understand the photon-induced charge carrier behaviors in those nanostructures, which determines the overall efficiency of the solar devices [43]. For a successful exciton dissociation, fast charge transportation, or slow charge recombination is required. In a variety of studies, inorganic shells and organic ligands are used to modulate the rate of the charge transfer. Lian et al. reported that the photon-generated excitons in CdSe/CdS quasi-type II core/shell QDs enables ultrafast charge separation and ultraslow charge recombination [44]. Furthermore, enhanced multiexciton dissociation efficiency can be achieved through interfacial electron transfer $[35,45,46]$. The photoinduced electron transfers from QDs (CdS, CdSe, and $\mathrm{CdTe}$ ) to molecular acceptors (anthraquinone, methylviologen, and methylene blue) have also been studied. The rate of the transfer increases as the size of the QDs decrease.[47]

Over the past decade, organic ligands that either withdraw electrons from or donate electrons (i.e., withdraw holes) to the QD cores, have drawn great attentions to researchers [31, 48-52]. Ligands with aromatic rings are especially interesting due to their piconjugation structure which offers the strong ability to extract photon-generated charge carriers from the QDs surface [49, 53-55].

Weiss et al. reported a method for controlling the size of an exciton within the QDs through a hole-delocalizing ligand phenyldithiocarbamate (PDTC) [56-58]. Para-substituted derivatives of the ligand with various electron-donating/withdrawing abilities were studied, which reveals that strong electron withdrawing group on the ligand expands the wave function of the excitonic hole in the CdSe QDs (Figure 1.10.a), and facilitates the hole transfer rate from the QDs [56]. Weiss and coworkers suggest that the energy level 
alignment between the QDs valence band and the ligand HOMO level plays an essential role for the hole transfer rate (Figure 1.10.b).[56]

a) Hole-delocalizing PDTC ligands with para-substituted $-\mathrm{OCH}_{3}$ and $-\mathrm{CF}_{3}$ group

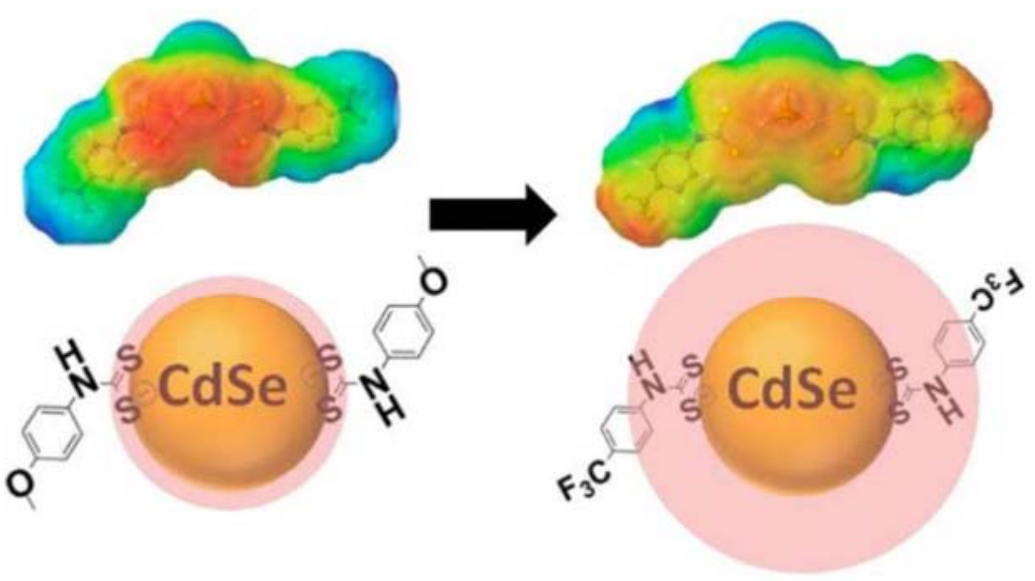

b) Energy level alignments between QD valence band and ligands HOMO levels

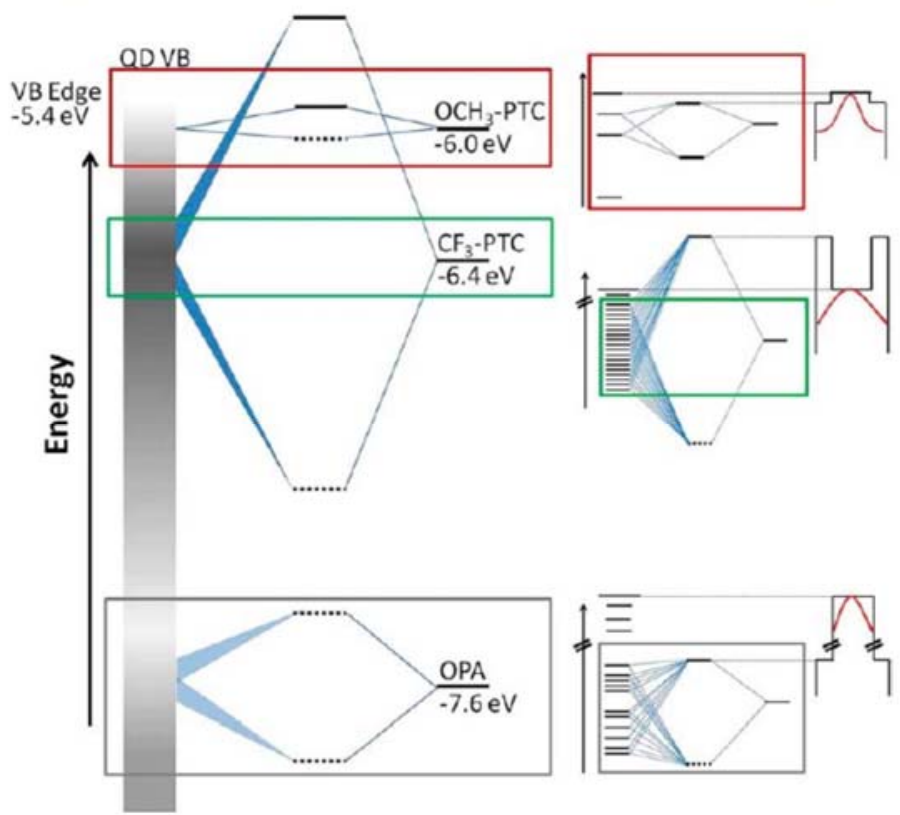

Figure 1.10. Wave function (a) and Energy Level Alignments between the Holedelocalizing Phenyldithiocarbamate (PDTC) Ligands and the CdSe QDs

Figure adapted with permission from reference 56. Copyright 2012, American Chemistry Society. 
Understanding of the charge transfer behaviors across the core/shell interface, or from QD core to its passivating ligands, as well as investigation on the charge recombination rate and its pathways, are important for the design of photovoltaic devices, and the improving of the efficiency of QD solar cells.

\subsubsection{Advantages of Implementing Quantum Dots in Photovoltaic Devices}

In general, QDs can be implemented in solar cells in various ways to improve the stability and PCE performance: (A) QDs can be used to form an ordered three dimensional array that acts as a photo-electrode to convert sunlight to electricity; (B) QDs can substitute for dye molecules in a dye sensitized solar cell as the light absorber, and generate a quantum yield greater than 100\% through MEG; (C) In QD dispersed solar cells, QDs are dispersed and blended with organic semiconductor polymers, to improve light harvesting efficiency [59].

Due to quantum confinement and other related properties, QDs possess some unique advantage when implemented in solar cells.[60]

i. Larger solar spectrum coverage. Band gaps of QDs can be tuned by changing their sizes. It is therefore possible to harvest photon energy across the entire solar spectral on the earth surface from UV (350 nm, i.e., $3.5 \mathrm{eV})$ to IR (2500 $\mathrm{nm}$, i.e., $0.5 \mathrm{eV}$ ) by mixing QDs with different sizes, and using them as the light absorber in photovoltaic devices;

ii. Higher efficiently due to MEG. Multiple exciton generation in QDs can be utilized to harvest photon energies much higher than the band gap energy, and 
hence bypass the Shockley-Queisser Limit. An external quantum efficiency exceeding $100 \%$ may be achieved through MEG;

iii. Tunable charge carrier dynamics. Inorganic shells and organic ligands can be designed into the synthesis of QDs, and the surface chemistry of QDs can be modulated according to various applications. Furthermore, hot carrier relaxation dynamics could be significantly reduced due to the exciton confinement effect;

iv. Commercially available. Solution-based fabrication of QDs solar cell is relatively cheap, while High-quality heterojunction/tandem solar cell with hole conductors is possible;

v. Thermally Stability. Compared to organic polymers, QDs are more stable and oxidation/moisture/UV resistant.

\subsection{Quantum Dots Synthesis and Surface Chemistry}

In 1981, Russian physicist Alexey Ekimov [61] discovered a semiconductor nanocrystals (known as quantum dots) in a glass matrix. In 1985, Louis Brus, professor of chemistry at Columbia University, observed QDs in a colloidal solution [62]. By 1993, colloidal CdX $(X=S, S e, T e)$ based QDs were successfully synthesized [63-66]. Within the past two decades, a variety of synthesis methods for QDs were developed and the optical properties of those QDs were studied [29, 60, 67-70]. Meanwhile, the commercial implementations of QDs have also been explored [71, 72].

For most QD-based applications, a crucial requirement for the synthesized QDs is that the size distribution must be narrow. Two steps are involved in the formation of monodisperse QDs: a rapid nucleation, followed by a slow growth period [63, 73, 74]. The rate of the nucleation is controlled by the critical temperature and the degree of 
supersaturation of the solution. Because of the high surface-to-volume ratio of the QDs, surface-passivating reagents known as surfactants need to be added during the synthesis of QDs in order to prevent aggregation [75]. Common surfactants include oleic acid (Omega9 fatty acid), dodecylamine (DDA), trioctylphosphine oxide (TOPO), and dodecanethiol (DDT). These surfactants can be used to tune the activity of the precursors [76], and the optical properties of the synthesized QDs [77].

Hot injection is the most common method for QDs synthesis. This method involves a rapid injection of the precursor(s) into a boiling surfactant, at the critical temperature. Upon injection, the precursor(s) decompose into monomers which initiates the nucleation step. Meanwhile, the overall reaction temperature starts to decrease rapidly. The decrease of temperature terminates the nucleation stage and the solution enters the slow growth period (Figure 1.11) [78]. The size of the synthesized QDs is controlled by the rate of the nucleation, the concentration of the precursor(s), the injection temperature, and the growth temperature, and the length of the growth period $[79,80]$.

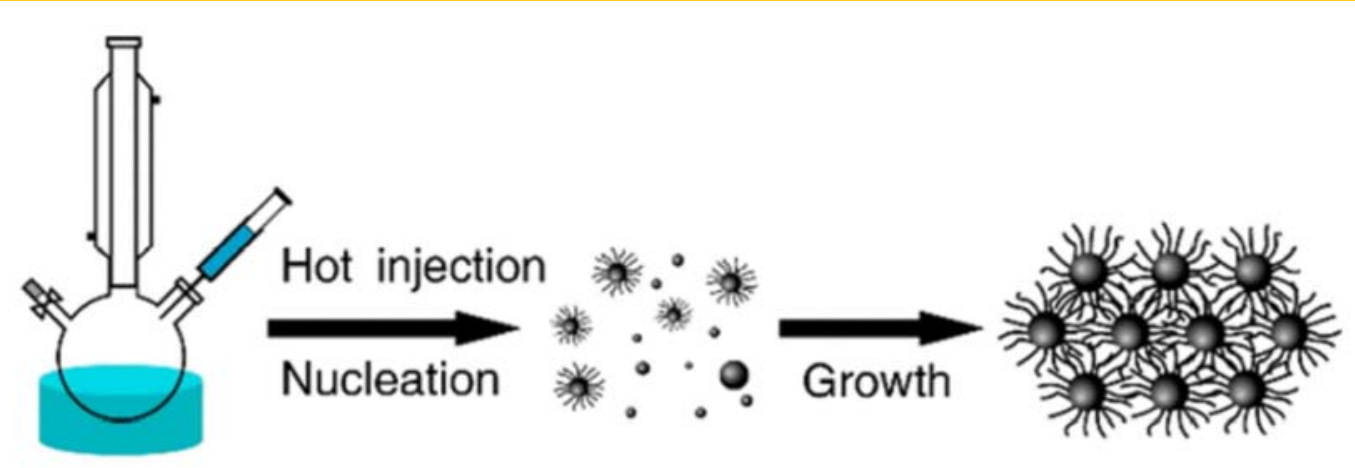

Figure 1.11. Hot Injection Method to Synthesize Quantum Dots.

Reprinted with permission from reference 70. Copyright 2015 American Chemistry Society. 
Other synthesis methods, such as the heat-up method [81], do not require the injection of the precursors, but involve a steady heating process of a mixture of the precursors and the ligands. In the recent years, a low-temperature synthesis method was reported. Using a cold injection method, very small "magic-sized" $\mathrm{Cd}_{34} \mathrm{Se}_{34}$ can be produced [82-84]. The diameter of the CdSe nanocluster is less than $2 \mathrm{~nm}$, leading to a larger band gap than conventional QDs $(d=2-10 \mathrm{~nm})$.

Purification procedures are required after the synthesis of the QDs, in order to remove the unreacted precursor(s) and excess surfactants. Otherwise, those impurities in the synthesized QDs will hinder the charge transfer processes and reduce the efficiency of the QD solar devices. As-synthesized QDs undergo a repeated processes of precipitation and dispersion in a non-solvent and a solvent, respectively, to remove unwanted impurities [85].

To implement QDs in photovoltaic devices, post-purification processes, both physical process and chemical process, are usually employed. The most common physical process is converting the QDs solution into a solid film by deposition methods, such as dropcasting, spin-coating or dip-coating. Chemical processes generally include ligand passivation, ligand exchange, alloying and doping, and the formation of a second material shell.[70]

\subsubsection{Ligand Exchange}

By varying the surface chemistry, the optical and physical properties of QDs can be modulated. As-purified QDs are typically capped with long chain surfactants. These surfactants act as an insulating medium, and stabilize colloidal QDs from aggregation in low-boiling-point organic solvents. However, long organic chains increase the spacing 
between QD molecules and lead to a low packing density, which hinder the charge transfer processes in the solar devices [86]. The initial capping can be exchanged from long organic ligands to short conductive ligands before device fabrications. Numerous studies showed that as the length of the ligands decreases, the observed inter-particle spacing is reduced, the electron transportation is facilitated, and the carrier mobility is increased.[87-90]

Adjusting surface ligands will significantly change the optical and electronic properties of QDs [91]. The choice of ligands will impact the charge transfer processes [92], and also determine whether a single QD species acts as a p-type or a n-type semiconductor in the solar devices [93-95].

\subsubsection{Core-Shell Quantum Dots}

Surface ligands can slowly desorb from the surface of the QDs. Thus ligandpassivated QDs need to be stored under low-temperature condition and handled with care. A more efficient surface passivating method is creating a long-lasting semiconductor shell by surrounding the core structure of the QDs with a secondary material, and forming a core/shell structure. This semiconductor shell will isolate the core material completely from the surrounding environment.

Two parameters effect the choice of the shell materials: the lattice mismatch and the band gap offset. Depending on the band gap offset, different types of core/shell structure was defined [96]. Type-I core/shell structures are formed when the shell material has a larger band gap than the core material, and the valence band edge of the shell is lower than of the core, while the conduction band edge of the shell is higher than of the core (Figure 1.12.a). In this case, the charge carriers are confined in the core structure, and therefore the optical 
properties of the QDs are depended on the core material. $\mathrm{PbS} / \mathrm{CdS}$ is the most studied QDs with type-I core/shell structures [97-99]. The $\mathrm{PbS} / \mathrm{CdS}$ QDs are synthesized through a cation-exchange procedure in which purified $\mathrm{PbS}$ QDs are mixed with a cadmium precursor [97]. The thickness of the CdS shell is optimized by controlling the conditions of the cationexchange to provide near-completed surface passivation [100].

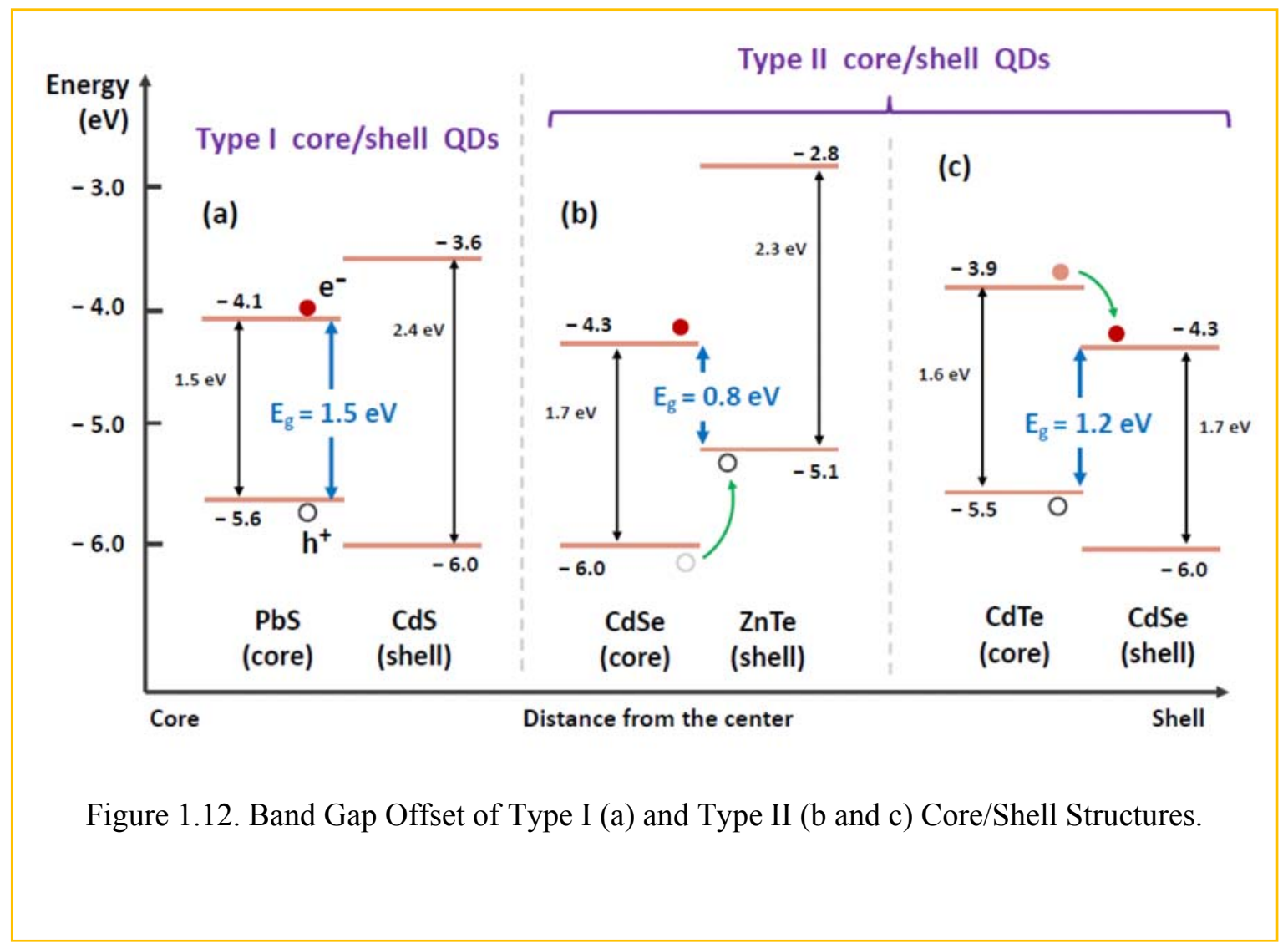

In type-II core/shell QDs, the band gap of the core material accommodates either the valence band edge or the conduction band edge of the shell material. When the valence band edge of the shell is within the band gap of the core material, the electrons stay in the conduction band of the core, while the holes transfer to the valence band of the shell (Figure 1.12.b). When the conduction band edge of the shell is within the band gap of the core, the electrons transfer to the conduction band of the shell material, while the holes stay in the core (Figure 1.12.c). In both cases, the band gap energy of the QDs red-shifts to longer 
wavelength (i.e., the band gap gets smaller). Electrons and holes are separated spatially as one charge carrier is localized in the core structure and the other carrier is localized in the shell structure, leading to a reduced electron-hole overlap and a longer exciton radiative lifetimes [101-103].

\subsubsection{Deposition Methods}

In order to transform the synthesized colloidal QDs into a solid thin film for the use of photovoltaic devices and other applications, different deposition methods were developed over the past decade. All methods rely on depositing a small volume of QDs solution onto a substrate, followed by solvent evaporation. The most common substrate for photovoltaic devices is titanium dioxide $\left(\mathrm{TiO}_{2}\right)$ coated fluorine-doped tin oxide (FTO) glass. Low boilingpoint solvent is typically used for a fast drying step, which facilitates a rapid and reproducible production.

The three most commonly employed deposition methods are drop casting [104], spin coating [105] and dip coating [106]. Drop casting is the simplest method. A drop of the QDs solution is deposited on the substrate and the solvent is left to dry without any further process. If the solvent has a high boiling point, drop casting can also be performed on a hot surface at elevated temperature. The disadvantage of the this method is the long drying time which can lead to a low packing density of the film [107].

Spin coating is the most common method. The substrate is fixed on a rotating stage that is under vacuum. Once the QDs solution is deposited onto the substrate, the stage starts spinning at a speed of $1000-1500 \mathrm{rpm}$ (rotation per minute). High quality and uniformed thin film can be produce through this method by using a constant spinning speed. The 
thickness of the film also can be adjusted by repeating the deposition-spinning process several times (named layer by layer spin-coating deposition method) $[105,108,109]$.

Dip casting method is performed by "dipping" the substrate into a stock solution of QDs, and withdrawing at a constant speed by an automated hand [106]. Due to the viscosity and the surface tension of the solution, a thin layer of the QDs is deposited on the substrate. While drop casting method is usually employed with high boiling-point solvent, the dip casting method only requires low boiling-point solvent such as hexane for a proper deposition.

\subsection{Other Applications of Quantum Dots}

As the synthesis technique of QDs become increasingly advanced during the past two decades, high quality colloidal QDs with various structures and compositions are largely available. The unique physical, electronical and optical properties of QDs are exploited as a novel material in numerous fields of applications.

QDs-based light emitting diode (QLED) has drawn intense research interests and inspired commercialization efforts [71]. Compared to organic LEDs (OLED) [110], QLED has a narrower emission peak, which is advantageous for high quality image in electroluminescence applications. Furthermore, semiconductor inorganic materials have a better thermal stability than organic materials. Due to the quantum confinement effect, QLED can be made to emit more than three premium colors and enable IR emission.

Early QDs-based electroluminescence devices were made of self-assembled QDs, and blue-green emission peaks were observed [111]. Later, QLED with multicolor emission was developed for image-displaying applications and light sources [112-116]. Near-Infrared 
emission from QLED was also achieved with $5 \mathrm{~nm}$ monodispersed PbSe QDs [88, 117] or 4.6 nm HgTe QDs [118]. By using layer-by-layer deposition method, multilayer QLED was fabricated where QDs are sandwiched between polymer composite hole transfer layer (HTL) and electron transfer layer (ETL) $[119,120]$. This type of organic/inorganic hybrid LED structure exhibits high luminescence for the whole visible spectrum [115].

QDs have also been implemented in the photodetector [121-123] and photoluminescence (downconversion) applications [124, 125]. QDs-based photodetectors with high sensitivity [126] or detection range that go into the mid-infrared [127] were fabricated in the last decade. Very recently, the first colloidal QDs photodetector with a nanoscale sensitivity was developed by using color-tunable plasmonic nanofocusing lenses [128]. Meanwhile, LED with nearly full color emission (i.e. white light) has been achieved using QD/polymer composites. Furthermore, CdSe/ZnSe QDs can be used for the downconversion of high energy (blue or ultraviolet light) to lower energy (red or green light) [129].

Due to the unique optical properties, QDs have the potential of being vastly employed in the field of bioimaging. Compared to traditional dye-based imaging, QDs have higher extinction coefficients and high quantum yields. Both absorbance and emission of the material can be tuned by size. The emission peak is narrow and multiple QDs can be used in the same assay without interference. Therefore, a significant amount of research is focused on using QDs in biological imaging.[130-132]

Researchers developed QDs-based methods for in vivo and in vitro imaging and diagnostic of live cells by functionalizing different size CdSe QDs with different molecules [133-135]. The size-tuned luminescent color offers great complement or replacement of conventional organic dyes. Fluorescence bioimaging, labeling and sensing techniques can 
also benefit from QDs implementations [136-138]. QDs with high quantum yield are prefect for intensity-based imaging, while the narrow emitting spectrum makes QDs ideal candidates for spectral-based applications. The longer fluorescence lifetime of QDs is advantageous for the use of lifetime or time-gated modes of biosensing and biolabeling.

More complex bioimaging techniques have also been developed, such as Fluorescence Resonance Energy Transfer (FRET) assay [139-141] where luminescent CdSe-ZnS core-shell QDs are used as the energy donors, and Surface Enhanced Raman Spectroscopy (SERS) [142-144] where biocompatible and nontoxic QDs are used for in vivo tumor targeting and detection.

One of the most important and advanced tools in medical imaging is Magnetic Resonance Imaging (MRI) technique. Based on the principle of proton nuclear magnetic resonance (NMR), protons get excited with a short radio frequency pulse, followed by a free induction decay relaxation which is measured/deconvoluted with Fourier Transform. Water and lipid molecules with high proton density appear brighter on MRI, while bone and tendon with low proton density appear darker. However, where there are soft tissues and regions containing air pockets and fecal matter, the traditional MRI technique falls short. It has been proven that QDs-based contrast agents can change the signal intensity of MRI at the desired location, which provides unprecedented sensitivity and selectivity for molecular imaging.[145-148]

\subsection{Motivation of the Study}

The functioning of modern human society is highly depended on the energy we consume. The majority of the world's energy sources rely on unsustainable fossil fuel, which 
is not only facing limited supplies, but also causes severe health and environmental issues leading to global warming concerns. Because the world population is unlikely to stop growing within the next fifty years, the energy crisis that we will encounter in the near future requires immediate action. Renewable energy source such as solar energy is the best alternative eco-friendly choice to meet the world's energy demands. For the past two decades, photovoltaic devices that transfer sunlight to electricity attracts enormous research interests. Among all the semiconductor devices, QD solar cells are the most advantageous due to its high photon to electron ratio (i.e. the quantum yield) and size-controlled tunable band gap.

Further improvement of PCEs of QD solar cells requires in-depth and quantitative understanding of photon induced charge carrier dynamics in QDs. This includes the generation and dissociation of excitons, charge transfer processes, and recombination of electrons and holes. In general, slow electron-hole recombination and a lowered charge transfer barrier are desired. Single-junction QD photovoltaic devices have been studied intensively over the past decades. The new focus in the field is multiple-junction integration. The development goal is to create high-efficiency solar cells with minimum loss in charge carrier thermalization and improved spectral utilization. It is also appealing to combine the distinctive properties of QDs with traditional solar cell materials such as silicon, or novel large-gap solar materials like perovskites, in order to develop new generation of solar devices with improved sunlight harvesting ability and enhanced efficiency. Studying the energy level structure and charge carrier dynamics of QDs is essential for all QD-based applications including solar cells. 
The works reported in this dissertation focus on the charge transfer kinetics in 2.9 $\mathrm{nm}$ CdSe QDs and $1.6 \mathrm{~nm} \mathrm{CdSe} \mathrm{nanoclusters.} \mathrm{These} \mathrm{nanoparticles} \mathrm{were} \mathrm{passivated} \mathrm{with}$ highly conjugated phenyldithiocarbamate (PDTC) ligands, which delocalize the charger carriers in the QDs. By using femtosecond laser spectroscopy, we studied the charge transfer processes and obtained important information of not only the interaction between PDTC ligands and $\mathrm{CdSe}$ nanoparticles but also the charge transfer across the interface. We uncovered sub-picosecond hole transfer and hot electron transfer processes when the CdSe nanocluster were excited with a photon energy much higher than the band gap. We also studied the role of passivating ligands semi-quantitatively by exchanging PDTC with its derivatives and hence tunning the energy level alignment between the HOMO level of the ligand and the hole level of the QDs. Differences in the energy level structure and charge transfer time constants were observed when various ligands were used. Understanding the fundamental principles behind how charge carriers relax and transport between the semiconductor core and organic/inorganic ligands can provide useful insights into these interesting and promising systems and guide the development of QD solar cells and other devices.

\subsection{Outline of this Dissertation}

The focus of Chapter 2 of this dissertation is the principle of ultrafast laser spectroscopy. Contents such as how the laser works, how the femtosecond laser pulse generates, and how the frequency of the laser converts to the desired frequency in order to meet experimental needs, are all described in separate sections in chapter 2. Chapter 2 also deals with the detailed experimental information of the ultrafast laser system, including 
schematic diagrams of the optical setups, water cooling and temperature control units, optical and electronic setups for the data acquisition, and the routine maintenance of the system. Chapter 3 focuses on the energy level alignment and charge transfer process between the molecule-like $1.6 \mathrm{~nm}$ CdSe nanocluster and its highly conjugated phenyldithiocarbamate (PDTC) ligands. Using pump-probe transient absorption spectroscopy, energy levels between the PDTC ligand and the CdSe nanocluster are compared. With the help of MathCAD global fitting, the charge transfer time components were determined. In Chapter 4, various para-substituted PDTC derivatives ligand-passivated CdSe nanoclusters are compared and studied. Copyright permissions, $\mathbf{C}$ programing source codes for the group velocity correction, and MathCAD codes for the global fitting, are all presented in the appendixes. 


\section{CHAPTER 2}

\section{ULTRAFAST TRANSIENT ABSORPTION LASER SPECTROSCOPY}

Ultrafast laser spectroscopy is an essential and powerful tool for the study of photovoltaic materials including quantum dots, semiconductor nanostructures, and organic polymers, as well as photovoltaic devices. It uses ultrashort laser pulses in the scale of picosecond $\left(10^{-12} \mathrm{~s}\right)$ to femtosecond $\left(10^{-15} \mathrm{~s}\right)$, to examine photo-induced dynamics in molecules and materials. In this chapter, we focus on the principle and experimental details of ultrafast laser spectroscopy, with emphasis on a femtosecond laser system and a transient absorption (TA) spectrometer used in our lab. The laser system consists of a Ti:Sapphire chirped pulse amplifier (CPA), and frequency conversion units, including two non-collinear optical parametric amplifiers (NOPAs), second harmonic generators (SHGs), and supercontinuum white-light (WL) generation. The TA spectrometer uses an optical pumpprobe configuration which can be set up in single-wavelength probing mode or white-light probing modes through two sets of flipping mirrors.

The first section of this chapter, Section 2.1, will describe the principle behind ultrashort pulse generation. The aforementioned frequency conversion techniques will be described in Section 2.2. Characterization of laser beams and laser pulses are important to ultrafast spectroscopy and will be discussed in Section 2.3. The TA pump-probe spectroscopic method will be described in Section 2.4. Details of the experimental apparatus 
including schematic diagrams are given in Section 2.5, while details of the detection and data acquisition systems will be given in Section 2.6. Section 2.7 is dedicated to spectral analysis procedures including group velocity dispersion (GVD) correction, convolution of transient kinetics by the instrument response function (IRF), as well as spectral simulation and global fitting. The last section (Section 2.8) of this chapter will briefly describe the routine maintenance of the laser system.

\subsection{Femtosecond Lasers and Amplifiers}

In ultrafast laser spectroscopy, ultrashort laser pulses are used to measure chemical dynamics and kinetic processes. There is no standard definition of the "ultrashort pulse". However, in most literature, the "ultrashort pulse" refers to an electromagnetic pulse in the IR, visible, or UV regions with a pulse duration ranging from tens of femtoseconds (fs) to hundreds of picoseconds ( $\mathrm{ps}$ ). Picosecond laser pulses are usually generated by active mode locking, while femtosecond pulses are generated by passive mode locking [149]. Laser pulses with a duration of less than a femtosecond, which is in the attosecond $\left(10^{-18}\right)$ regime, are also possible to achieve by high harmonic generation [150]. Attosecond laser pulses generally are too fast for measuring chemical processes and therefore less useful for photovoltaic research. On the other hand, pulses with a duration between $1 \mathrm{fs}$ and $1 \mathrm{~ns}$ are most suitable for studying chemical reactions as well as photo-induced processes in photovoltaic and photo-emissive materials. The 1999 Nobel Prize for Chemistry was awarded to Ahmed H. Zewail [151], for his contributions on introducing ultrashort pulse to chemical reaction observations and the opening a new field of femtochemistry [152]. 


\subsubsection{Principle of Lasers}

In 1887, Heinrich Hertz [153] discovered the photoelectric effect, a phenomenon in which electrons or other free carriers are emitted when light is shone onto a material. In 1905, Albert Einstein [154] proposed the quantum theory of light, an idea that a beam of light is not a wave but a collection of discrete wave packets, which he called "photons", each with an energy $h v$. Einstein's theory links Hertz's previous discovery of the photoelectric effect with Max Planck's discovery of the Planck relation $(E=h v)$, which explains that the photons of a light have a quantized energy, and the energy is proportional to the frequency of the light. In the photoemission process, an electron absorbs the energy of one photon, and gain enough energy to overcome the electron binding force, and is emitted from the atom as a free particle. The kinetic energy of the emitted electron does not depend on the intensity of the incoming light but only the photon energy, (i.e., frequency of the light). For the photoelectric effect, observation of the photoelectron emission is only possible when the photon energy of the light reaches a certain threshold. The hole left behind by the photoelectron can give rise to Auger effect, which leads to spontaneous emission or stimulated emission. Spontaneous emission is the process in which the electron from an excited energy state and the hole at a lower energy state (e.g., ground state) are recombined and emit a quantum of energy in the form of a photon. Stimulated emission is the process that an incoming photon with a specific frequency induces the electron-hole recombination, and creates a new photon with identical phase, frequency, polarization, and direction of the initial photon $[155,156]$.

A laser is a device that uses spontaneous emission and stimulated emission to emit light coherently, hence the name: light amplification by stimulated emission of radiation. A 
laser device must consist of three things for proper light amplification and laser emission: a gain medium, population inversion of electrons, and an optical feedback system, which most commonly is in the form of a laser cavity.

The gain medium is a material (in the form of gas, liquid, solid or plasma) with properties that allow it to amplify a specific wavelength of light by stimulated emission. For light amplifications and the stimulated emission to happen, the gain medium need to be put into an excited state by an external source of energy. The external energy is typically supplied as an electric current (in the case of the diode laser), or as light at a different wavelength (when pumped by a flash lamp, or another laser).

As the gain medium absorbs the energy, and uses it to excite some electrons to the higher-energy quantum states, spontaneous emission will occur. The emitted photon then induces stimulated emission in the gain medium. Most lasers start via spontaneous emission, then work by stimulated mission during continuous operation. When the number of electrons in the excited state exceeds the number of electrons in the lower-energy state, population inversion is achieved. In this case, the amount of stimulated emission due to incident light is larger than the amount of absorption, i.e., gain $>$ loss. Hence, the light is amplified.

The third essential component of a laser is an optical feedback, in the form of an optical cavity, or in other case, an electronic oscillator. The laser cavity consists of two or more high-reflective mirrors on either end of the gain medium. Emitted photons from the gain medium bounce back and forth in the laser cavity between mirrors, being amplified each time they are passing through the gain medium. One of the cavity mirrors is partially transparent, called output coupler, which allows a certain portion of the intracavity light to exit the cavity and to be used as the output beam of the laser devices.[157, 158] 


\subsubsection{Brief History of Lasers}

In 1917, Albert Einstein first explained the theory of stimulated emission, which later became the fundamental principle of lasers. Einstein stated that, when the population inversion exists between the upper and lower electron energy levers in the atomic system, it is possible to achieve amplified stimulated emission which gives the same frequency and phase as the incident radiation. In the late 1940s and 1950s, scientists and engineers such as Charles Townes, Joseph Weber, Alexander Prokhorov and Nikolai G Basov, pioneered the works towards the first realization of a device that uses Einstein's theory of stimulated emission to amplify microwave, i.e., a MASER (Microwave Amplification by Stimulated Emission of Radiation). Later, scientists like Townes, Arthur Schawlow, and Gordon Gould proposed the realization of an optical maser, i.e., a laser, which can create powerful beams of visible light and infrared. In May 1960, Theodore Maiman at Hughes Research Labs demonstrated the first working laser by using ruby as its gain medium and optically pumping with commercial flash lamps. In the next two decades, almost all major types of lasers were invented which introduced not just laser spectroscopy, but a completely new type of systems with potential applications in a wide variety of fields.[159-161]

\subsubsection{Types of Lasers}

There are many types of lasers that are employed for research, medical, industrial, and commercial uses. Commonly, lasers are classified based on their gain medium: solid state, gas, excimer, dye, and semiconductor.[157, 158]

A solid state laser uses a solid gain medium which consists of glass or crystalline as the host material, to an added "dopant" such as neodymium, chromium, ytterbium, or 
titanium. The most common solid state lasers include neodymium-doped yttrium aluminium garnet (Nd: YAG) lasers, ruby $\left(\mathrm{Cr}_{\mathrm{A}} \mathrm{Al}_{2} \mathrm{O}_{3}\right)$ lasers, and titanium-doped sapphire $\left(\mathrm{Ti}_{\mathrm{i}} \mathrm{Al}_{2} \mathrm{O}_{3}\right)$ lasers, The laser emits light at different wavelength based on its solid state materials.[162]

For gas lasers, Helium and Helium-Neon $(\mathrm{HeNe})$ are the most common gain mediums, which have a primary output of a visible red light. Excimer (combined word of Excited and Dimer) lasers use reactive gases such as chlorine and fluorine mixed with inert gases as argon or xenon. The gas phase active medium is electrically stimulated, which produces an excited dimer for light emission.[162]

Dye laser is a liquid phase laser using complex organic dyes as the lasing media. Based on the absorption properties of the dye, dye lasers are highly tunable over a broad range of frequency. Last, semiconductor lasers, or sometimes called diode lasers, use a p-n junction of a semiconductor diode as the active laser medium. The most common semiconductor material is Gallium Arsenide (GaAs). Semiconductor lasers can be very small in size and appearance.[157, 162]

Lasers can also be characterized by the duration of the laser emission, e.g., continuous wave (CW) lasers, or pulsed lasers. A CW laser emits a continuous laser beam with a stable average output power, while a pulsed laser generates output beam with a pulse duration of few femtoseconds to few milliseconds. CW lasers are usually pumped by a flash lamp or a diode laser, while pulsed lasers are achieved through techniques such as Q-switch, and mode locking. A Q-switched laser is a pulsed laser that contains a shutter device which does not allow emission until the shutter is opened. Energy is built up and then released to produce a single intense laser pulse whenever the shutter is opened. Mode locking is another method to produce pulsed laser beams. Mode locked lasers operates as a result of the 
resonant modes inside the optical cavity. As the phases of different frequency modes inside the cavity are synchronized, i.e., locked, the interference between all modes produce a train of regularly spaced pulses, each having a duration of few femtosecond to few picosecond. The principle of the mode locking will be further discussed in the next section.[157, 162]

\subsubsection{Generation of Ultrashort Laser Pulses}

While Q-switch can be used to produce millisecond and microsecond laser pulses, and the generation of attosecond pulses is relied on high harmonic generation process, the generation of ultrashort laser pulses (in the range of femtosecond and picosecond) is achieved by mode locking techniques.

In short, the basis of mode locking techniques is to induce a fixed-phase relationship between all longitudinal modes in the laser resonant cavity. In a simple Fabry-Perot laser cavity, light travels as a wave and bounces between mirrors of the cavity. As the light constructively and destructively interferes with itself, a series of standing waves, or "modes", is formed between the mirrors. These modes, known as the longitudinal modes, have a discrete set of frequencies that are dependent on the length of the cavity. Multiple longitudinal modes occur as long as their wavelength (frequency) satisfy the resonance condition based on the length of the cavity $(\boldsymbol{L})$ : the wavelength of mode $\lambda=\mathbf{2} \boldsymbol{L} / \mathbf{n}$, where $\mathbf{n}$ is an integer known as the mode order. The total number $(\mathbf{N})$ of longitudinal modes that can occur in the cavity is dependent on the gain profile of the laser active medium [163].

When a laser is "mode-locked", those longitudinal modes inside the laser cavity no longer oscillate independently, but interfere with each other constructively and maintain a fixed-phase relationship between all modes. The result is a train of equally spaced intense 
pulses with repetition rate related to the cavity round trip time. The time between two pulses $\Delta \mathbf{t}=\mathbf{2} L / \mathbf{c}$, where $\boldsymbol{L}$ is the length of the cavity, and $\boldsymbol{c}$ is the speed of time. The time duration of each pulse $(\tau)$ is determined by the value of "N":

$$
\tau=\frac{0.441}{N} \times \frac{2 L}{c}
$$

The broader the gain profile of the laser active medium is, the larger the value of "N" will be, and therefore the shorter the duration time of the laser pulses. For this reason, Titanium $\left(\mathrm{Ti}^{3+}\right)$ doped Sapphire $\left(\mathrm{Al}_{2} \mathrm{O}_{3}\right)$, i.e., Ti:Sapphire crystal, is the most common solidphase material used for ultrashort pulse generation, due to its broad gain bandwidth [164].

As shown in Figure 2.1, the calculated gain (dashed red line) of Ti:Sapphire crystal has a very broad peak ranging from $700 \mathrm{~nm}$ to $1100 \mathrm{~nm}$, with a central wavelength at $\lambda \approx$ $800 \mathrm{~nm}$. This broad gain profile is essential for the generation of femtosecond pulse due to the inverse relationship of the time duration of a pulse and its frequency bandwidth. The spectral (frequency bandwidth) and temporal (duration of a pulse) properties of the laser are mathematically related through Fourier Transform and therefore obey the Heisenberg uncertainty principle [165], making them conjugate duals. And due to this conjugative relationship, materials with a broad gain profile are chosen to generate ultrashort laser pulses. 


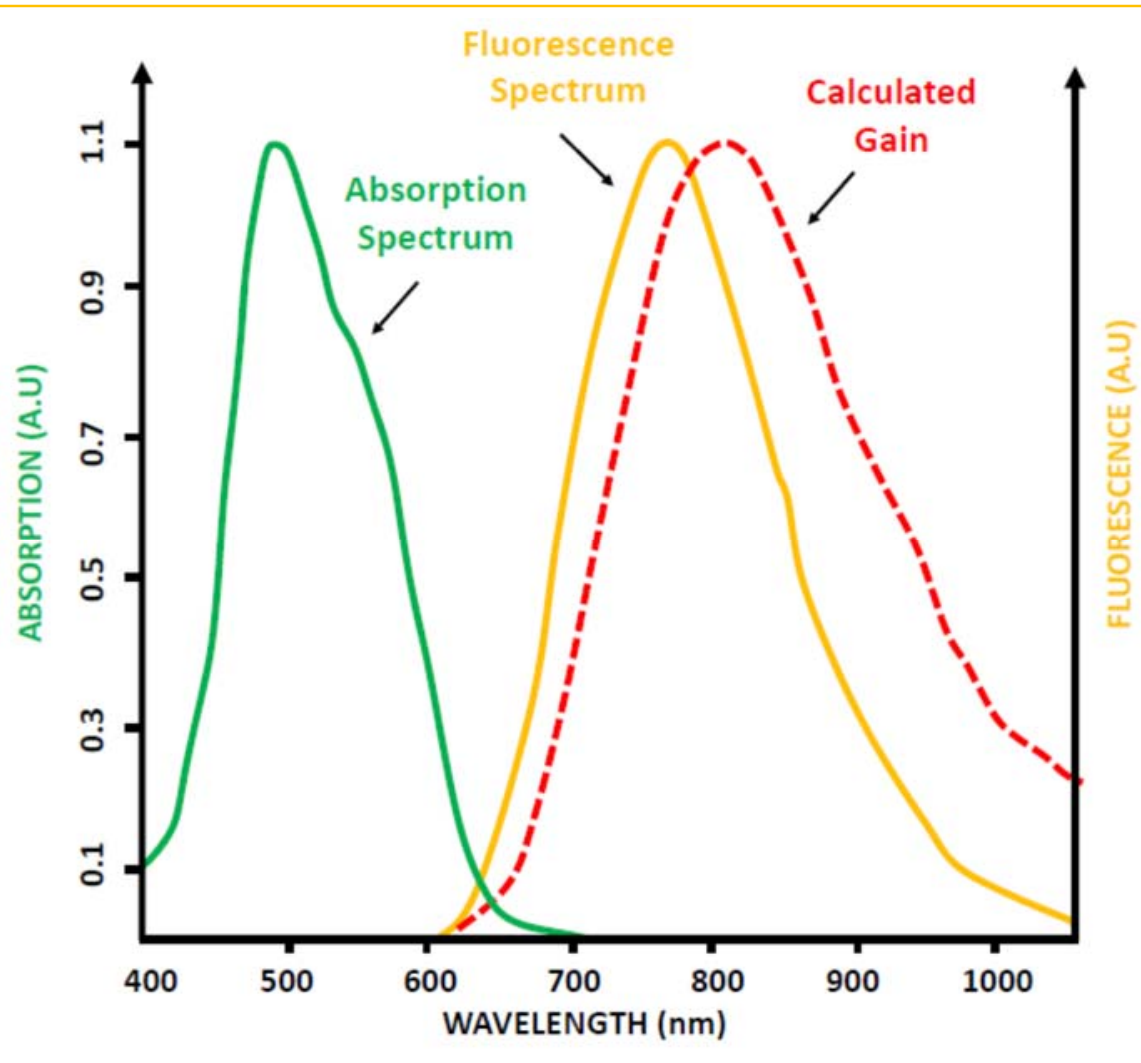

Figure 2.1. Absorption and Fluorescence Spectrum of Ti:Sapphire Crystal.

Dashed line is the calculated gain profile (Gaussian line shape) of Ti:Sapphire.

For example, the gain bandwidth of the HeNe Laser is quite narrow [166], only about $1.5 \mathrm{GHz}$ with a center wavelength at $633 \mathrm{~nm}$. With a typical cavity length of $50 \mathrm{~cm}$, there are only 8 longitudinal modes that can oscillate simultaneously in the cavity, which generates a laser pulse with a pulse duration of 293 picosecond [167]. On the other hand, a Ti:Sapphire laser with $94 \mathrm{THz}$ (Figure 2.1, FWHM = $200 \mathrm{~nm}$ ) bandwidth with a center wavelength at $800 \mathrm{~nm}$, the pulse duration can be as short as 4.68 femtosecond [167]. Evidently, this is just the shortest pulse duration time that can be achieved based on the property of the gain medium. In a real mode-locking Ti:Sapphire laser, the pulse duration is effected by many other factors such as the overall dispersion in the cavity. RP Photonics 
Encyclopedia [167] provide great calculation tools for the duration time of Gaussian shape laser pulses, based on its conjugative relationship with the gain medium bandwidth.

Due to its narrow shape on the time domain, ultrashort pulse has extremely high peak intensity. For example, the peak power of a laser pulse with $1 \mathrm{~mJ}$ energy and $10 \mathrm{~ns}$ duration time is only 0.1 megawatt (MW), while with the same energy $(1 \mathrm{~mJ})$ but $100 \mathrm{fs}$ pulse duration time, the peak power of the pulse would be 10 gigawatts (GW).

When high intensity ultrashort pulses propagating in the gain medium, nonlinear optical processes such as Kerr-effect will occur. Kerr-effect $[163,168,169]$ is the phenomenon that the refractive index (n) of a material increases as the electric field, i.e., the intensity $(\boldsymbol{I})$, of the light that travels through the medium.

$$
n=n_{2} I+C
$$

This variation of the refractive index only becomes significant with high intensity beam, therefore is very common in ultrashort pulse generation. Kerr effect induces the socalled Kerr-lens inside the laser medium, which leads to other nonlinear optical effects such as self-focusing (SF), self-phase-modulation (SPM), and Kerr-lens mode-locking (KLM) [170]. SPM leads to a spectral broadening of the laser pulse, which is essential to the ultrashort pulse generation, as we discussed earlier [149, 171, 172]. More details about Kerr effect and other optical nonlinearities will be discussed in Section 2.2.

\subsubsection{Chirped Pulse Amplification (CPA)}

When amplifying the ultrashort pulses, the intense peak power may leads to destruction of the gain medium. Therefore, chirped pulse amplification (CPA) method [173] is almost always employed to prevent damaging the optics. In the CPA method, the 
ultrashort pulses are "chirped" and temporally stretched to a longer duration through dispersion. This pulse stretching reduces the peak power of the pulses. The stretched pulses are fed into the regenerative amplifier that consists of an optical cavity, an active medium with broad gain profile, and a pump laser that is used to pump the active medium. After the amplification, a dispersive compressor is used to remove the chirp and temporally compresses the amplified pulses to its original duration time (as similar to the input pulse). This whole process (as shown in Figure 2.2.b) is hence named chirped pulse amplification.

When Ti:Sapphire crystal is used in the regenerative amplifier as the active medium, frequency-doubled Nd:YAG laser (lasing at 1064/2 =532 nm) is often chosen to be the pump laser, based on the absorption profile of the Ti:Sapphire crystal $(500-550 \mathrm{~nm}$, as shown in Figure 2.1).

\subsubsection{Femtosecond Lasers in the Present Work}

The femtosecond laser pulses used in the present work are generated by the ClarkMXR CPA-Series laser head. This CPA laser head is built on two levels (Figure 2.2.a) and consists of a diode laser, a SErF (single-mode Erbium doped fiber) fiber oscillator, a pulse stretcher, a frequency doubled Nd:YAG pump laser, a Ti:Sapphire regenerative amplifier, and a pulse compressor.

The diode laser is located at the bottom level, and is a fiber-coupled solid-state laser with an output wavelength of $980 \mathrm{~nm}$ and a power of $217 \mathrm{~mW}$. The diode current (in $\mathrm{mA}$ ) and the diode power (in $\mathrm{mW}$ ) are shown on the LCD screen of the fiber laser electronics control board which is located on the side of the CPA laser head. 
a) Inside of the CPA Laser Head

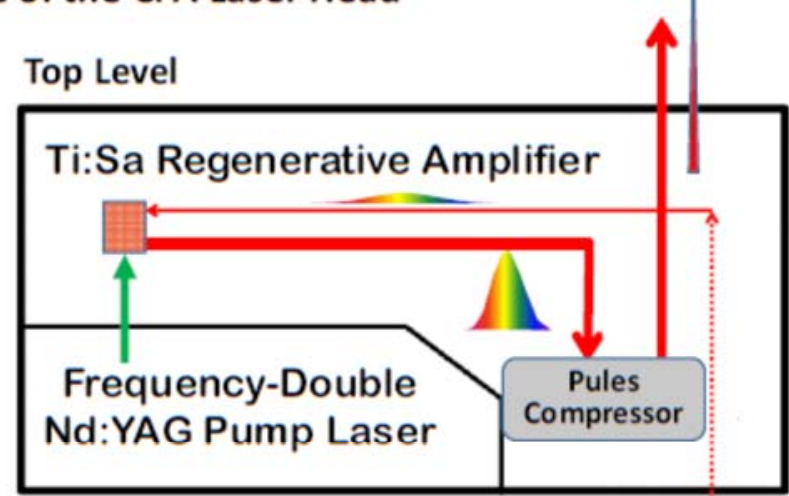

Bottom Level

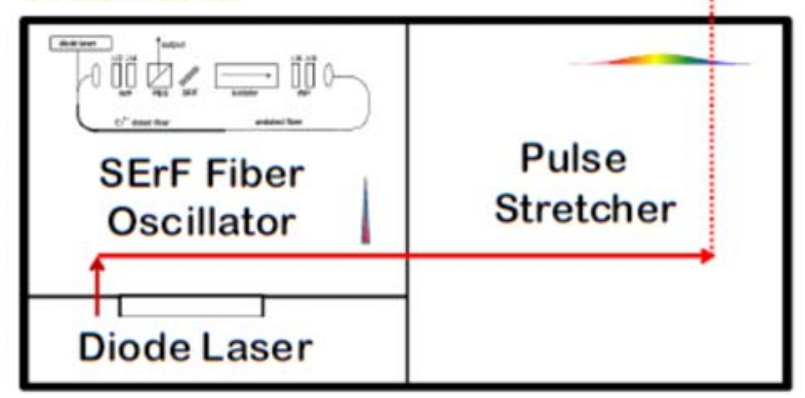

b) Scheme of Chirp Pulse Amplification (CPA)

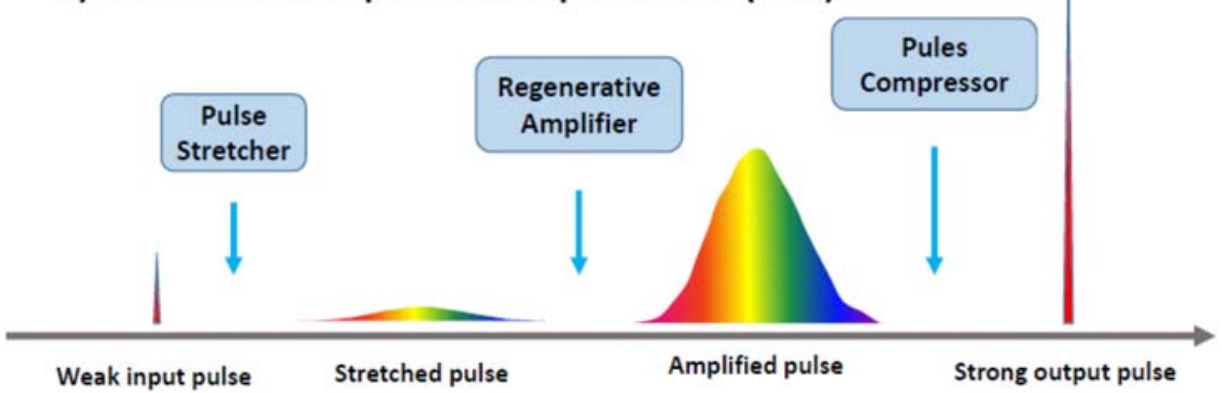

c) SErF fiber laser

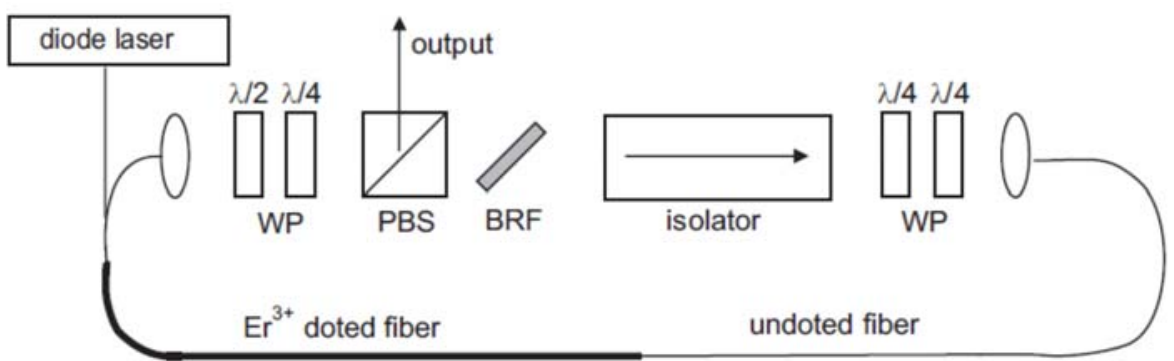

Figure 2.2. Schematic Diagram of the Components in CPA-Series Laser Head

There are two levels (a) in the CPA laser head, the bottom level is consist of a diode laser, a SErF fiber laser (c), and a pulse stretcher. The top level is consist of a frequency doubled Nd:YAG laser, a Ti:Sapphire regenerative amplifier, and a pulse compressor. The output of the SErF is amplified through the chirp pulse amplification (CPA) scheme (b). 
The output of the diode laser is used to pump the SErF fiber laser. The SErF fiber oscillator is a fiber ring laser (Figure 2.2.c) that uses Erbium $\left(\mathrm{Er}^{3+}\right)$ doped fiber as gain medium. The cavity of the fiber ring laser consists of the $\mathrm{Er}^{3+}$ doped fiber (acts as the gain medium), the undoped fiber (acts as additional Kerr medium), a wave plate (WP), a polarization beam splitter (PBS), a birefringent filter (BRF), an optical isolator, and the output coupler. The output of the SErF fiber oscillator has a wavelength of $775 \mathrm{~nm}$, and a pulse energy of less than $1 \mathrm{~nJ}$.

Since the laser diode is telecommunications grade and has a lifetime of 20 years, the SErF fiber laser is recommended to be left on at all times to achieve optimal performance [174]. The internal water-cooling system is utilized to stabilize the temperature of the SErF fiber laser at $20 \pm 1{ }^{\circ} \mathrm{Celsius}$.

The output power of the SErF fiber laser is amplified by the CPA method (Figure 2.2.b). First, the output pulse (duration time $=100 \mathrm{fs}$ ) is stretched to $20 \mathrm{ps}$ by a grating pair. Then, the stretched pulses are fed as the seed into the regenerative amplifier (RGA) cavity on the top level. The Ti:Sapphire regenerative amplifier is pumped by a Q-switched frequency-doubled Nd:YAG laser which in turn is pump by a flash lamp. The flash lamp is cooled by the external water-cooling system whenever the system is on.

The Q-switched is triggered at $1 \mathrm{kHz}$ by the master signal sent from a Pockels cell driver. The Pockels cell is an electro-optic device that is serving as the essential component in the Q-switch [175]. The Pockels effect, named after German physicist Friedrich Pockels, explains the principle of the Pockels cell: an applied constant or variable voltage (i.e., electric field) to the crystal will induce linear changes in the birefringence of the crystal 
[176]. Therefore, applying a constant voltage allows the Pockels cell to operate as a voltagecontrolled wave plate.

DT-506 Pockels cell driver controls the Q-switch of the YAG laser, and the injection time of seed pulse (into the Ti:Sapphire RGA) by monitoring the output of the SErF fiber laser. All to ensure the correct timing between the seed and the pump for maximum amplification of the RGA.

DT-506 contains three independent digital delay generators. Delay 1 controls the injection time of the seed, and Delay 2 controls the ejection time when the energy of the amplified pulses reaches saturation. Delay 3 is created as a convenience to the user for additional synchronization needs. The values of these three delays are set at installation, hence should not be changed regularly, and need to be recorded whenever they are changed. As 02/20/2017, the time delays of the DT-506 driver are: 05924, 06075, 0622e.

Figure 2.3 shows a "train" of the amplified pulses (also called "the regenerative trace") after the injection of the seed in to the Ti:Sapphire crystal. The intensity of the pulse increase every round trip inside the cavity. After four or five round trips in the cavity, the pulse energy reaches saturation, which is the optimum cavity dumping time. Therefore, the pulse is coupled out the cavity at saturation (pulse energy $=900 \mathrm{~mW}$ at $1 \mathrm{kHz}$ repetition rate), and then compressed to $150 \mathrm{fs}$ pulse duration time by a pair of diffraction gratings in the pulse compressor. The amplified and temporally compressed pulses exit the CPA laser head and is used as the fundamental of all further experiments. 


\section{a) Schematic Drawing of Amplified Pulse Train}
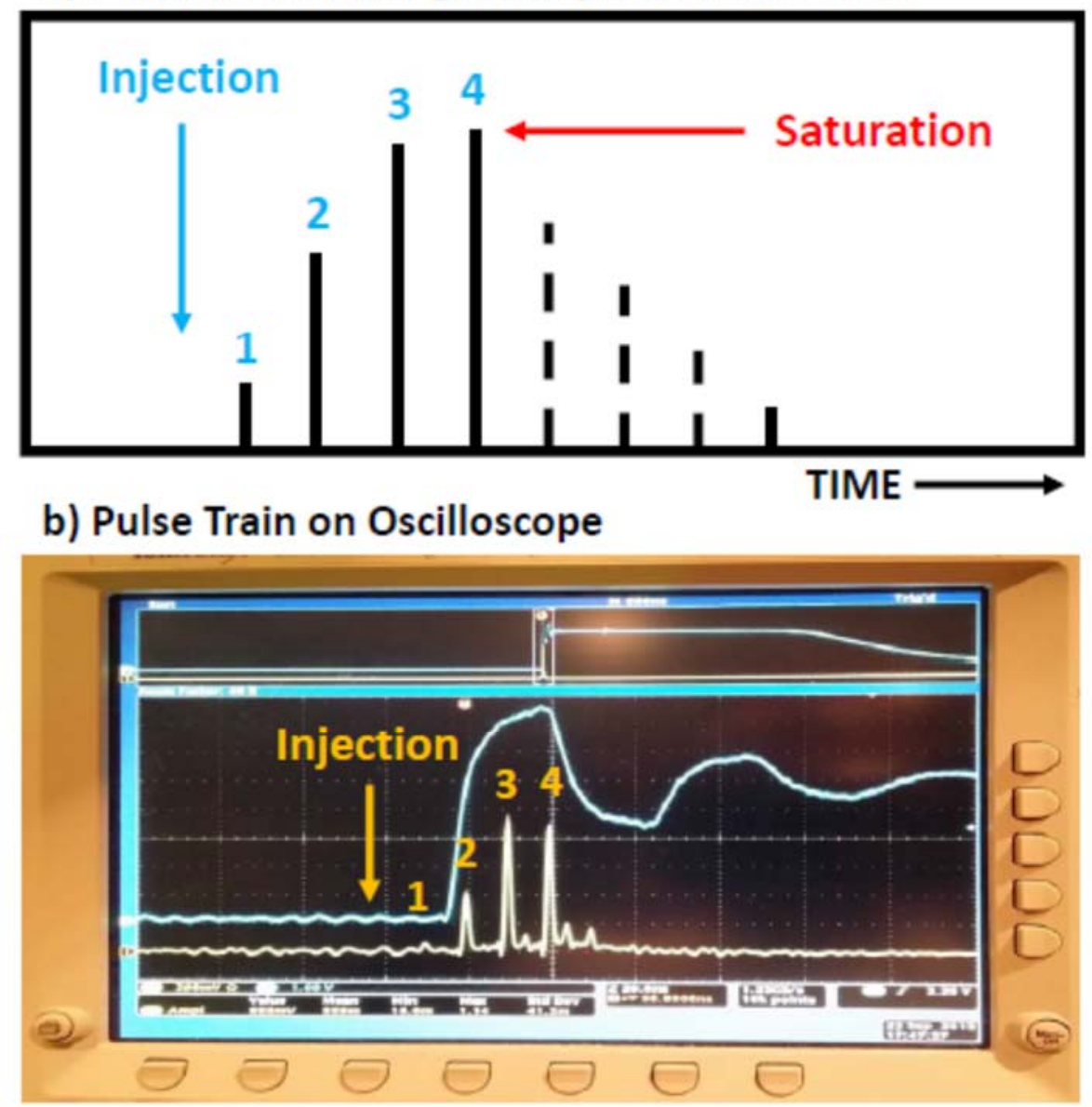

Figure 2.3. Pulse Train of the Ti:Sapphire Regenerative Amplifier.

(a) Regenerative trace of the CPA laser head (b) The amplified pulse is coupled out after the fourth round trip. Intensity of the first pulse is too low to be seen on the oscilloscope.

\subsection{Frequency Conversion Methods}

In the studies of femtochemistry, frequency conversions of the fundamental output are required in order to investigate the dynamics of the charge carries. Since the fundamental output of the CPA laser head is very hard to tune to the desired frequency, external frequency conversion methods such as nonlinear optical parametric amplification (NOPA), second harmonic generation (SHG), and supercontinuum generation (SG) are employed. The aforementioned frequency conversion methods, as well other frequency mixing processes, 
such as third harmonic generation (THG), optical parametric generation (OPG), optical parametric oscillator (OPO), supercontinuum generation $(\mathrm{SG})$, are all based on the nonlinear optical effect [170] that will be discussed in this section.

\subsubsection{Nonlinear Optical Processes}

Linear optics is a field of optics consisting of linear system which is used to explain phenomena when light enters lenses, mirrors, wave plates and diffraction gratings. Under linear system, the frequency of the output beam will be the same as the input beam. However, linear system only applies when the light intensity is low. In the case of femtosecond laser, the values of the electric field $(\boldsymbol{E})$ comparable to the interatomic electric fields is very high, commonly in the order of $10^{8} \mathrm{~V} / \mathrm{m}$. Therefore, nonlinear optical processes occur.

In general, nonlinear optical processes can be described as a Taylor Series expansion. The dielectric polarization density (dipole moment per unit volume) $\boldsymbol{P}(\mathrm{t})$ is expressed as [177] :

$$
P(t)=\varepsilon_{0}\left(\chi^{(1)} E(t)+\chi^{(2)} E^{2}(t)+\chi^{(3)} E^{3}(t)+\cdots\right)
$$

Where $\chi^{(\mathrm{n})}$ is the $\mathrm{n}^{\text {th }}$ order susceptibility of the optical medium, and $\boldsymbol{E}(\mathrm{t})$ is the electric field at time t. An $\mathrm{n}^{\text {th }}$ order of nonlinearity leads to ( $\left.\mathrm{n}+1\right)$-wave mixing. For example, a secondorder nonlinearity leads to a three-wave mixing [171]. The nonlinear polarization $\boldsymbol{P}$ for a three-wave mixing can be expressed as :

$$
P(t)=\varepsilon_{0} \chi^{(2)} E^{2}(t)
$$


So that for two incoming waves $\boldsymbol{E}_{\mathbf{1}}\left(\boldsymbol{\omega}_{1}\right)$ and $\boldsymbol{E}_{2}\left(\boldsymbol{\omega}_{2}\right)$ at frequencies $\boldsymbol{\omega}_{1}$ and $\boldsymbol{\omega}_{2}$, the second order of polarization $\boldsymbol{P}^{(\mathbf{2})}$ can be written as :

$$
\begin{aligned}
P^{(2)} & =\varepsilon_{0} \chi^{(2)}\left(E_{1}+E_{1}\right)^{2} \\
& =\varepsilon_{0} \chi^{(2)}\left[E_{1} \cos \left(\omega_{1} t\right)+E_{2} \cos \left(\omega_{2} t\right)\right]^{2} \\
& =\varepsilon_{0} \chi^{(2)}\left[E_{1}^{2} \cos ^{2}\left(\omega_{1} t\right)+E_{2}^{2} \cos ^{2}\left(\omega_{2} t\right)+2 E_{1} E_{2} \cos \left(\omega_{1} t\right) \cos \left(\omega_{2} t\right)\right] \\
& =\frac{1}{2} \varepsilon_{0} \chi^{(2)}\left[\begin{array}{l}
\left.E_{1}^{2} \cos \left(2 \omega_{1} t\right)+E_{2}^{2} \cos \left(2 \omega_{2} t\right)+2 E_{1} E_{2} \cos \left[\left(\omega_{1}+\omega_{2}\right) t\right]\right] \\
+2 E_{1} E_{2} \cos \left[\left(\omega_{1}-\omega_{2}\right) t\right]+\left(E_{1}^{2}+E_{2}^{2}\right)
\end{array}\right]
\end{aligned}
$$

There are total of five terms in this equational expression of $\boldsymbol{P}^{(\mathbf{2})}$. The last term represents that the induced polarization contains the two original components $\boldsymbol{E}_{1}$ and $\boldsymbol{E}_{2}$. The first four terms, however, represent three different nonlinear effect applications that the three-waving mixing corresponds to. When the light is emitted at a new frequency $\boldsymbol{\omega}$ : (a) If $\boldsymbol{\omega}=\mathbf{2} \boldsymbol{\omega}_{1}$ or $\boldsymbol{\omega}=\mathbf{2} \boldsymbol{\omega}_{2}$ (first and second term in Equation 2.5), two photons are absorbed, and a new photon at two times the frequency is emitted in a process known as frequency doubling, also called the second harmonic generation (SHG) (Figure 2.4.a); In a similar way, if the emitted photon has three times the frequency as the incident photon, frequency tripling, or third-harmonic generation (THG) occurs (Figure 2.4.b); (b) If $\boldsymbol{\omega}=\boldsymbol{\omega}_{1}+\boldsymbol{\omega}_{2}$ (third term in Equation 2.5), the new frequency of the emitted photon is the sum of the original frequencies, i.e. sum frequency generation (SFG) occurs (Figure 2.4.c); and (c) If $\boldsymbol{\omega}=\boldsymbol{\omega}_{1}-$ $\boldsymbol{\omega}_{2}$ (fourth term in Equation 2.5), difference frequency generation (DFG) occurs (Figure 2.4.d). 


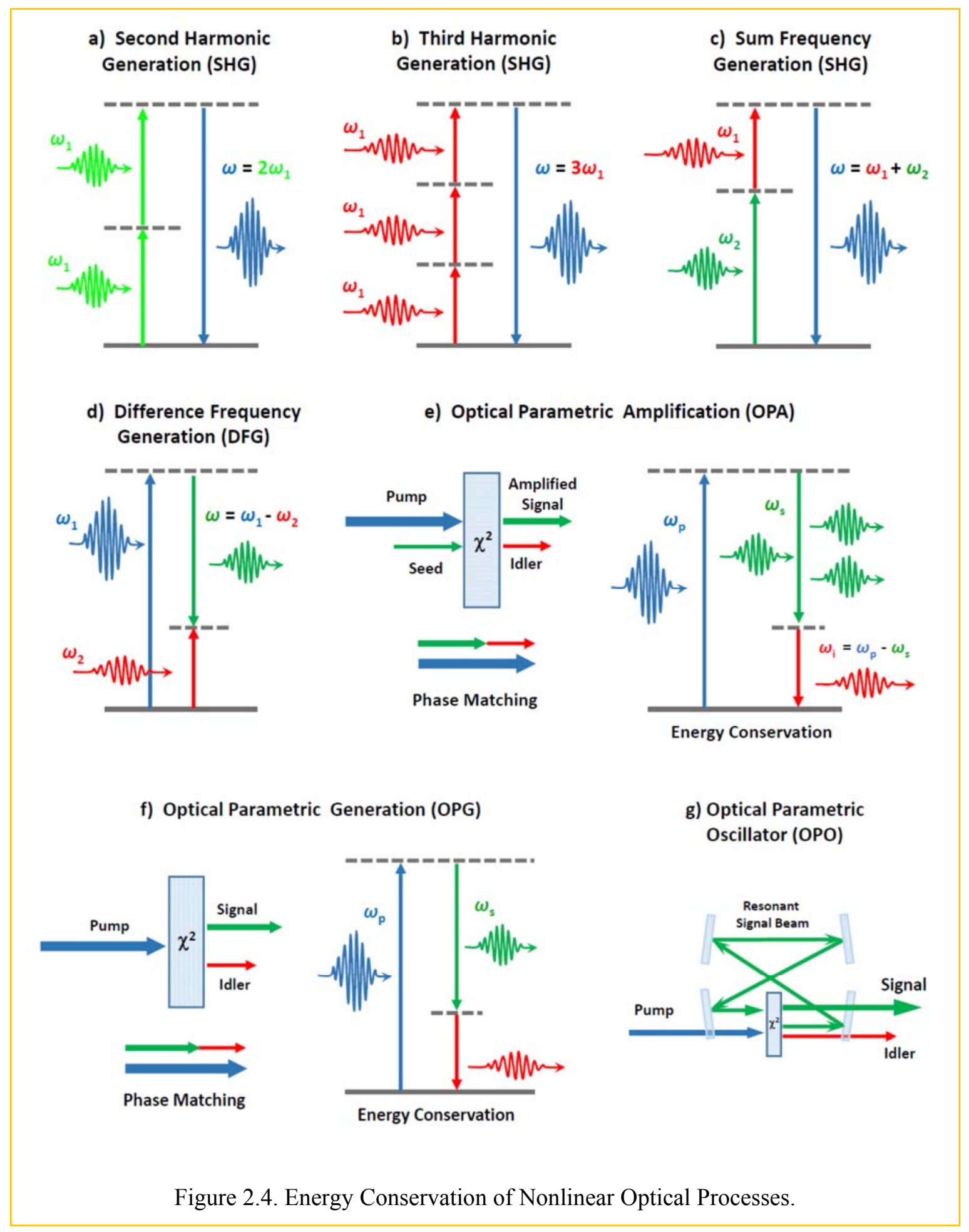


Optical parametric amplification (OPA) and optical parametric generation (OPG), are actually variations of the DFG, where the electronic field of $\boldsymbol{\omega}_{2}$ is much weaker, in the case of OPA, or completely absent, in the case of OPG.

In OPA process, two light beams at frequency $\boldsymbol{\omega}_{1}$ and $\boldsymbol{\omega}_{2}$ enter a nonlinear optical crystal, the higher frequency $\left(\boldsymbol{\omega}_{1}\right)$ beam is called the pump, while the lower frequency $\left(\boldsymbol{\omega}_{2}\right)$ beam is called the signal. In this process, one signal photon (at frequency $\boldsymbol{\omega}_{2}$ ) creates two photons with the same frequency through stimulated emission, with the help of the pump photon (at frequency $\boldsymbol{\omega}_{1}$ ), which excited one electron to a higher energy level. Therefore, the OPA process makes the pump beam weaker, while amplifying the signal beam. And OPA also generates a new beam (called the idler) at the frequency $\boldsymbol{\omega}=\boldsymbol{\omega}_{1}-\boldsymbol{\omega}_{2}$ through DFG. The pump and the signal photons usually travel collinearly through a nonlinear optical crystal, therefore the conservation of photon momentum (also known as the phase matching condition) is required for the process to work efficiently (Figure 2.4.e).

In OPG process, only one light beam, the pump, (at $\left.\boldsymbol{\omega}_{\mathrm{p}}\right)$ serves as the input source, and the output is two light beams, the signal and the idler, of lower frequency with the relationship of $\boldsymbol{\omega}_{\text {pump }}=\boldsymbol{\omega}_{\text {signal }}+\boldsymbol{\omega}_{\text {idder. }}$ Traditionally, $\boldsymbol{\omega}_{\text {signal }}$ is larger than $\boldsymbol{\omega}_{\text {idler, }}$ and both the photon energy conservation and the phase matching condition are required (Figure 2.4.f).

Optical parametric oscillator (OPO) is a light source that is based on the optical gain from OPA process in a nonlinear crystal. First a pump beam with high optical intensity and high spatial coherence enters the crystal, inducing OPG process which generating signal beam and idler beam. The signal beam then resonates in a series of mirrors and re-enters the crystal, inducing OPA process which amplifying the signal every times it pass through the crystal (Figure 2.4.g). 
The sum of the frequencies of the signal and the idler beam always equals to the frequency of the pump. However the exact frequencies of the signal and the idler are determined by phase matching conditions.

\subsubsection{Phase Matching Conditions}

In most transparent materials, like BK7 glass, frequency conversion via nonlinear optical processes will not work successfully, due to the destructive interference between the second harmonic wave and the original wave. For all the aforementioned frequency conversion methods, and other phase-sensitive nonlinear processes, constructive interference only occurs efficiently when the phase-matching condition [178] is satisfied:

$$
\vec{k}_{3}=\vec{k}_{1}+\vec{k}_{2}
$$

Where $\overrightarrow{\boldsymbol{k}}_{\mathbf{3}}$ is the wave vector of the output beam, and $\overrightarrow{\boldsymbol{k}}_{\mathbf{1}}$ and $\overrightarrow{\boldsymbol{k}}_{\mathbf{2}}$ are the wave vectors of the input beams (Figure 2.6.a).

Besides the phase matching condition and the conservation of energy, the output of nonlinear optical processes is also effected by the phase matching angle and the polarizations of the input beams.

Nonlinear optical processes such as the three-wave mixing only occur in birefringent crystalline material. Unlike common material, birefringent crystalline material has at least two indices of refraction, and Uniaxial is the simplest type of birefringence. A uniaxial crystal, has a single preferred axis called the extraordinary (e) axis, or the optical axis. While the other two principal axes which are perpendicular to the e axis are called the ordinary (o) axes. 
Light whose polarization that is parallel to the optical axis, (in another word, within the principal plane, which consists of the optical axis and the light propagation direction), sees the refractive index $\mathbf{n}_{\mathbf{e}}$ and is named the extraordinary (e) ray. For light whose polarization is perpendicular to the principal plane, it sees the refractive index $\mathbf{n}_{\mathbf{0}}$ and is named as the ordinary (o) ray (Figure 2.5.a).

In a three-wave mixing, if both input beams are "o" rays, and the output beam is "e" ray, type I (oo-e) phase matching occurs. If input beams have unequal polarization as “o” and " $\mathrm{e}$ " rays, respectively, and the output beam is "e" ray, type II (eo-e and oe-e) phase matching occurs (Figure 2.5.a).

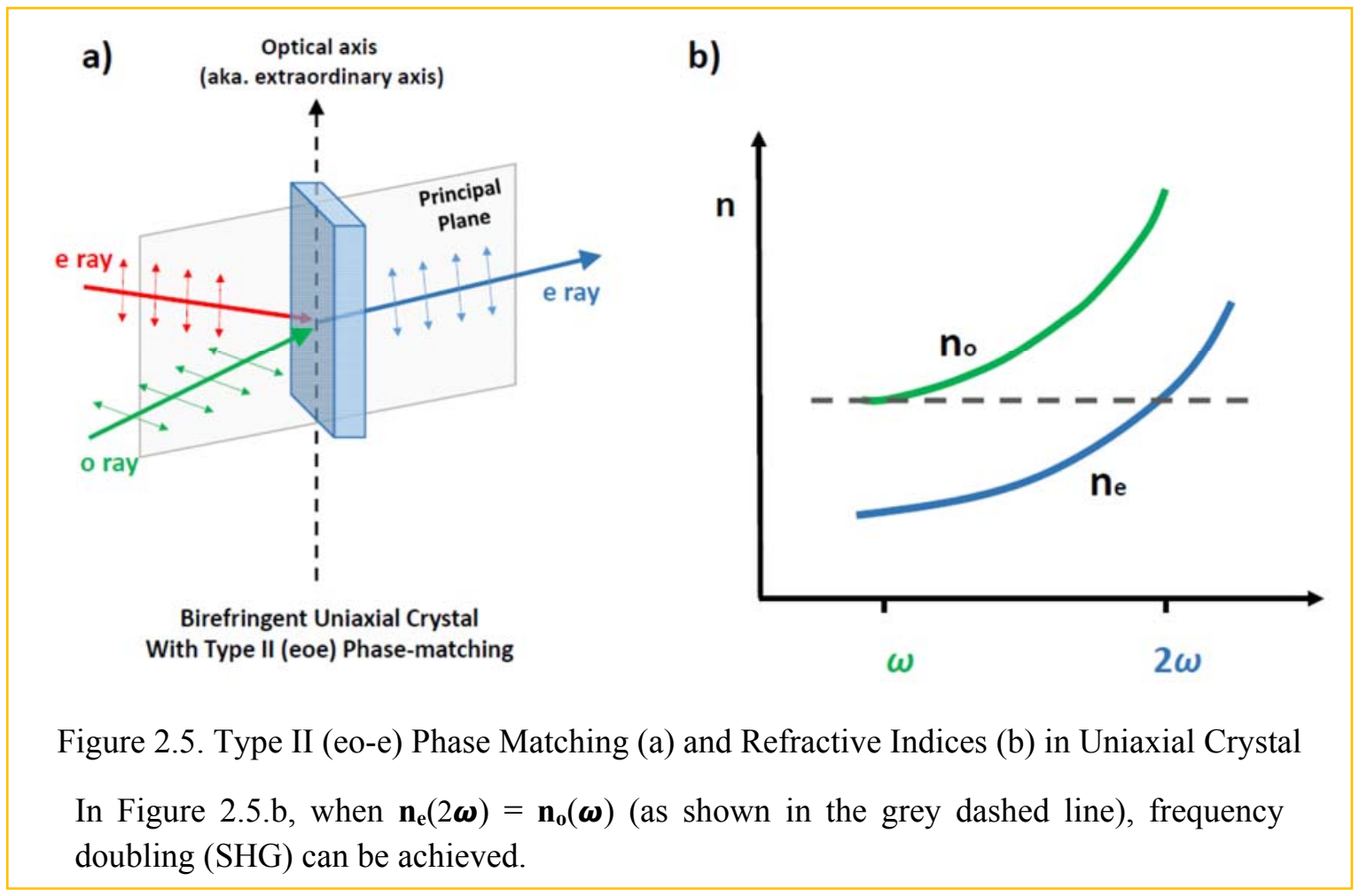

Another consideration for nonlinear optical processes is the phase matching angle. In regular materials, there is only one index of refraction which is dependent on the frequency. This makes $\mathbf{n}\left(\boldsymbol{\omega}_{1}\right)=\mathbf{n}\left(\boldsymbol{\omega}_{2}\right)$ unlikely. However, in birefringent materials, there are 
two indices of refraction $\mathbf{n}_{\mathbf{0}}$ and $\mathbf{n}_{\mathbf{e}}$. The value of $\mathbf{n}_{\mathbf{e}}$ is a function of the phase matching angle $(\theta)$, which is between the optical axis and the pump light propagation direction (Figure 2.6.c).

$$
n_{e}(\theta)=n_{o}\left(\frac{1+\tan ^{2} \theta}{1+\left(n_{o} / n_{e}\right) \tan ^{2} \theta}\right)^{1 / 2}
$$

When tuning this angle $\theta$ properly, $\mathbf{n}\left(\boldsymbol{\omega}_{1}\right)=\mathbf{n}\left(\boldsymbol{\omega}_{2}\right)$ can be achieved. In the case of the SHG process in our laser system, the incident beam is $\mathbf{o}$ ray and the output beam is $\mathbf{e}$ ray, when the phase matching angle is tuned to make $\mathbf{n}_{\mathbf{e}}(2 \boldsymbol{\omega})=\mathbf{n}_{\mathbf{0}}(\boldsymbol{\omega})$ (as indicated in grey dashed line in Figure 2.5.b), frequency doubling (i.e. the SHG) occurs.

SHG and NOPA are the two most common frequency conversion methods employed in our system. The schematic diagram of NOPA is shown in Figure 2.6. Unlike OPA, the pump and the seed of the NOPA do not travel through the crystal collinearly, hence, "nonlinear" optical parametric amplification. Both the pump and the seed (signal) beams are horizontally polarized, hence they are $\mathbf{e}$ rays, the idler beam is $\mathbf{o}$ ray, and therefore type-II $\beta$-barium borate (BBO) crystal is used. The angle between the pump and the optical axis of the crystal is the phase matching angle $\theta$. When the SHG of the fundamental $(388 \mathrm{~nm})$ is used as the pump source, supercontinuum "white light" is used as the seed source, and $\theta$ is tuned properly, the amplified signal can be obtained at the desired wavelength of the NOPA output $(532 \mathrm{~nm})$. 
a) Phase matching condition

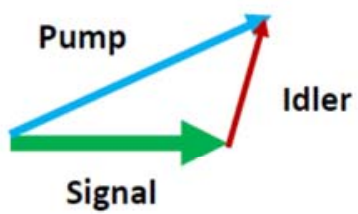

$\overrightarrow{k_{p}}=\overrightarrow{k_{i}}+\overrightarrow{k_{s}}$ b) Conservation of energy

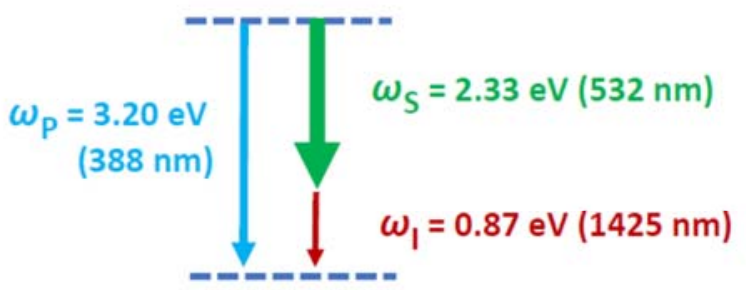

$\omega_{\mathrm{p}}=\omega_{\mathrm{s}}+\omega_{\mathrm{i}}$

\section{c) Nonlinear Optical Parametric Amplification with Type-II BBO Crystal}

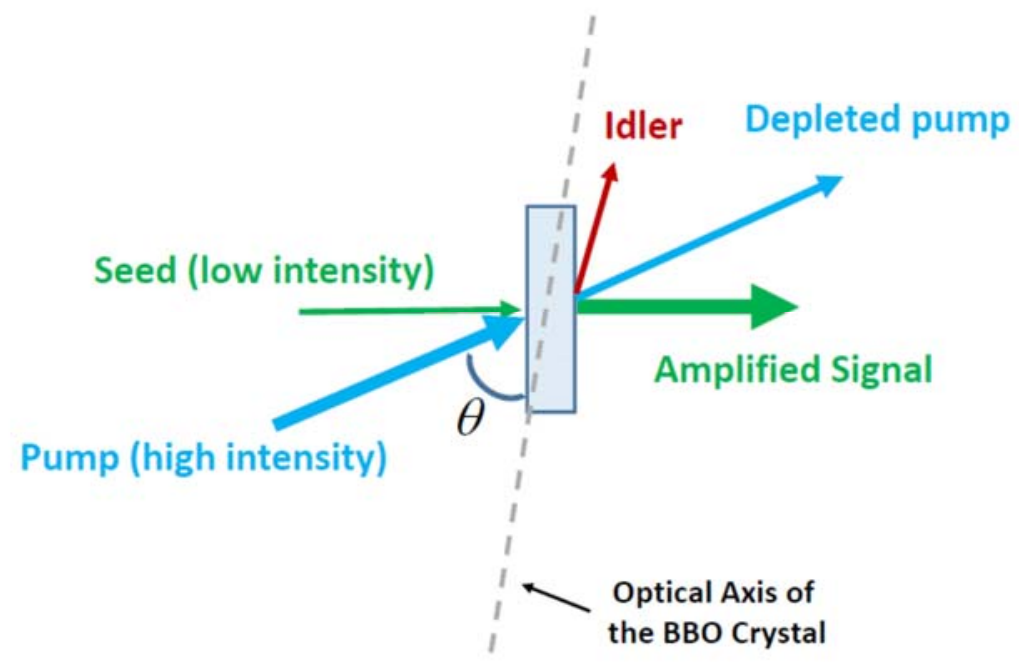

Figure 2.6. Schematic Diagram of Nonlinear Optical Parametric Amplification (NOPA)

\subsubsection{Supercontinuum Generation}

When an intensive monochromatic ultrashort laser pulse propagates through a transparent medium, a collection of nonlinear processes occurs. All those nonlinear optical effects act together to cause a series of spectral broadening of the original pulse, which results an ultrashort broadband pulse, ranging from UV to near IR. The resulting low- 
temporal-coherence but high-spatial-coherence pulse is called the supercontinuum "white light" [179].

The first picosecond supercontinuum generation (SG) was observed in liquid and solid by Alfano and Shapiro in 1970 [180], and later, femtosecond SG was obtained by Fork et al. in 1983 [181]. In our femtosecond laser system, the supercontinuum "white light" is used as the seed source of the NOPA, and as the probe beam in the pump-probe transient absorption scheme (TAPPS).

Even though SG has many applications in broadband absorptions [182], optical parametric amplifications and pulse compressions [179], optical communications [183], fluorescence lifetime imaging [184], gas sensing [185], and many more. The principal behind the generation of supercontinuum white light is still not perfectly understood. Researchers believe that strong self-phase modulation (SPM), self-focusing (SF), and stimulated Raman scattering are the most essential contributors. Other theories claim that cross-phase modulation, multiphoton excitation, excited Raman emission, and four-wave mixing also play important roles.[179, 180, 186]

As mentioned in Section 2.2.1, when a high intensity ultrashort laser pulse enters a transparent medium, the electric field (E) of the light is comparable in strength to the atomic field of the material, and the polarization of the nonlinear effect can be expressed by a Taylor Series expansion (Equation 2.3). The second-order term in the expansion leads to nonlinear effect such as three-wave mixing, second harmonic generation (which we discussed earlier). The third-order term contributes to processes such as four-wave mixing, SF, and SPM.

SF and SPM are the fundamental processes contributing to the supercontinuum generation, and they both are based on the intensity dependency of the refractive index, $\mathbf{n}(I)$, 
i.e. the Kerr-effect $[171,172]$. This optical Kerr-effect is derived from the above Tayler Series expansion $\mathbf{P}(\mathrm{t})$ equation and is given as:

$$
n(I)=n_{0}+n_{2} I
$$

Where $\mathbf{n}_{\mathbf{0}}$ and $\mathbf{n}_{\mathbf{2}}$ are constant, and $\boldsymbol{I}$ is the intensity of the incoming laser beam. Since the intensity profile of the incoming beam should be a Gaussian shape, i.e. the center of the beam tends to be more intense (larger $I$ ) than the edges, the center of the beam will see a higher refractive index $\mathbf{n}$ than the edges, and therefore travel slower, which causes the beam to self-focus to the center.

For SF to occur efficiently, two additional conditions need to be met for the incoming beam: (1) the beam need to be focused by a lens before entering the medium, a "jump start" for the SF process; and (2) the power of the beam need to exceed the critical power threshold:

$$
P_{c r i t}=\frac{3.77 \lambda^{2}}{8 \pi n_{0} n_{2}}
$$

SPM is a temporal equivalent to the SF process. As an increase in intensity will cause a red shift (Stokes-shift) in the laser frequency, and a decrease of the intensity will cause a blue shift (anti-Stoke-shift). The time variation in intensity is what contributes to the frequency-broadening effect of the original laser beam, which leads to supercontinuum generation. This creation of a variety of colors from a monochromatic light source, is further enhanced by the self-steepening of the pulse. Furthermore, stimulated Raman scattering, cross-phase modulation, and other nonlinear effects also occur alongside SPM which all contribute to the generation of the supercontinuum white light. Experimental setup details for white light generation that is used in this work are described in Section 2.5.3 Probe Pulse Generation. 


\subsection{Characterization of Ultrashort Pulses}

Ultrashort pulses need to be characterized to determine their spectral (center wavelength, band width, pulse chirping), spatial (beam diameter, convergence and divergence, beam quality), temporal (repetition rate, pulse duration), and energy (pulse energy, peak power, average power) properties.

The spectral characterization is relatively simple since the pulses are relatively broad in the wavelength domain. A simple charge coupled device (CCD) spectrometer can be used to monitor the instrumental outputs. The wavelength domain spectra for the output of the CPA laser head (red trace in Figure 2.7,), and the NOPA (green) are obtained by an Ocean Optics USB4000-VIS-NIR spectrometer.

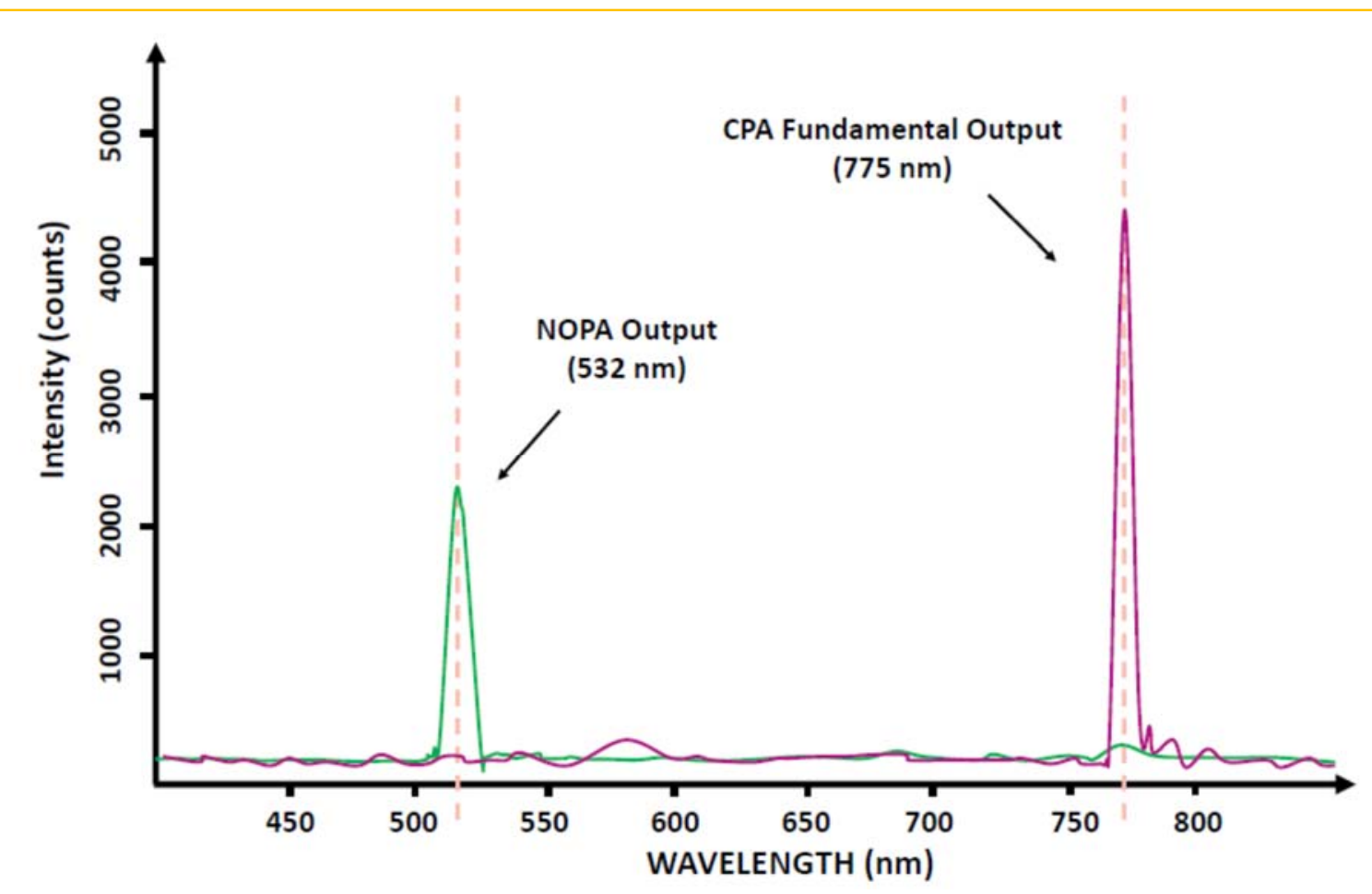

Figure 2.7. Spectra of CPA Fundamental (775nm) and NOPA Output (532 nm) 
However, the temporal characterization presents a problem, since the pulse duration of a femtosecond laser is much shorter than the response time of the fastest electronic devices. The limitations of photodiodes and oscilloscopes are commonly in the order of 200 fs, while the duration time of ultrashort pulses is sometimes only few fs. Therefore, several techniques are introduced to counter this problem. The most simple and commonly used technique to measure the pulse duration time is the optical autocorrelation [172].

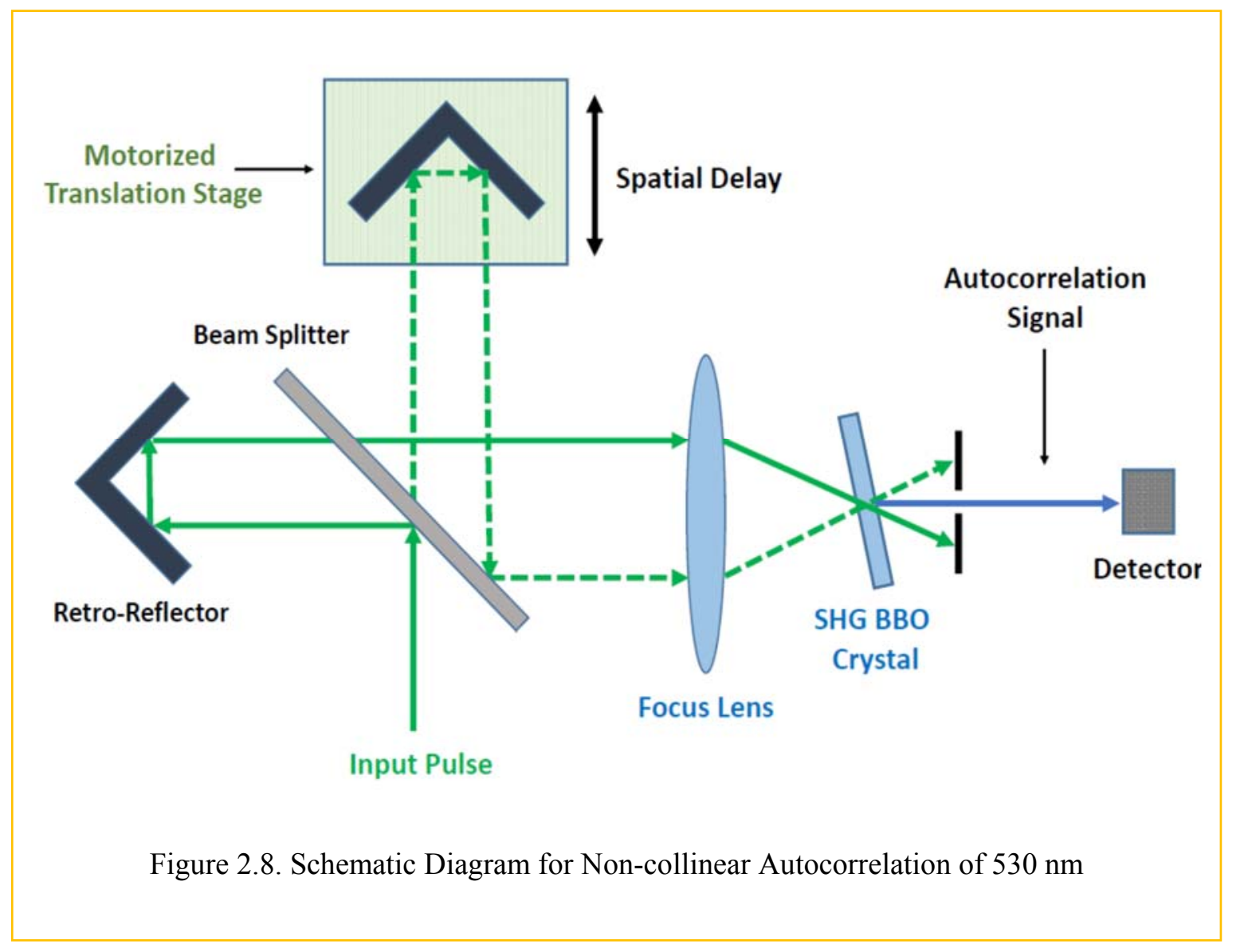

In optical autocorrelation, the original pulse (green arrow) is split into two identical beams by a beam splitter. Each beam is sent to a retro-reflector and the returning beams are overlapped in the SHG BBO crystal in a non-collinear geometry. One of the retro-reflectors is fixed on a computer-controlled motorized translation stage, which introduces a temporal 
delay between the two beams. The SHG signal, resulting from the interaction of the two beams in the BBO crystal, is measured by a slow detector (Thorlabs Si Biased Detector, Model \#: DET10A, Detection Range: $200-1100 \mathrm{~nm}$ ). Meanwhile, both transmitted beams, as well as the SHG signal from each of the individual beams, are blocked by an iris. The SHG signal measured by the detector is called the intensity autocorrelation signal (blue arrow in Figure 2.8).

Since the motorized translation stage is controlled by a computer, the temporal delay $(\tau)$ between the two beams can be determined based on the speed of light. (In the function of $\tau=2 \mathbf{d c}$, where the spatial difference in the beam paths is twice as much as the distance that the stage moved.)

The intensity autocorrelation signal $\boldsymbol{A}(\tau)$, produced by the nonlinear frequencydoubling process inside the BBO crystal, is a function of the time delay $\tau$ :

$$
A(\tau)=\int_{-\infty}^{+\infty} I(t) I(t-\tau) d \tau
$$

Therefore, plotting the signal recorded by the detector against temporal delay times, a Gaussian shape pulse can be obtained. After fitting the non-collinear autocorrelation signal with Gaussian line shape function, the full width at half maximum (FWHM) of the fitting is obtained and used to characterize the pulse duration of the original beam (Figure 2.9). 


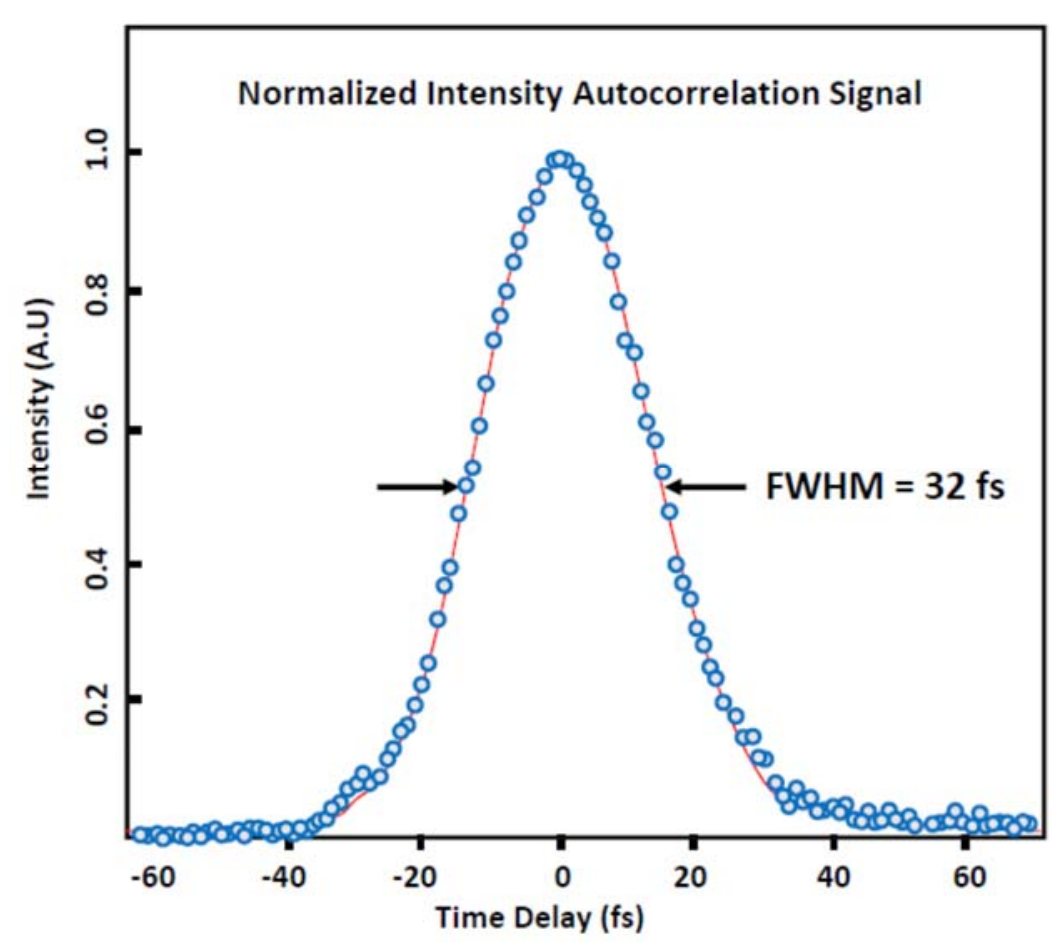

Figure 2.9. Intensity of Autocorrelation Signal Plotted Against Temporal Delay

The non-collinear autocorrelation can be used to obtain the information of the pulse duration. But in order to obtain the phase information, collinear autocorrelation technique needs to be employed. In collinear autocorrelation, two identical laser beams travel through the same path and interact in the BBO crystal. The SHG signals from each of the individual beams as well as the SHG of the interaction are recorded by the detector. In this case, the intensity of the autocorrelation signal is called interferometric autocorrelation $\boldsymbol{I}_{\boldsymbol{M}}(\tau)$ and is given in a function of :

$$
I_{M}(\tau)=\int_{-\infty}^{+\infty}\left|(E(t)+E(t-\tau))^{2}\right|^{2} d t
$$

Where $\boldsymbol{E}(\mathbf{t})$ is the complex electric field and corresponding to the intensity $\boldsymbol{I}(\mathbf{t})$ as : 


$$
I(t)=|E(t)|^{2}
$$

Although in theory, the phase information of the ultrashort pulse is available through collinear interferometric autocorrelation. In practice, the trace obtained is too complex and difficult to analyze with sufficient accuracy. Therefore in the present work, in addition to spectral characterization techniques, only non-collinear intensity autocorrelation is employed to obtain the temporal information of the femtosecond pulse.

Invented by Rick Trebino and Daniel J. Kane in 1991, Frequency-resolved optical gating (FROG) [187] is a spectrally resolved autocorrelation. Instead of only measuring the pulse duration like the old autocorrelation method does, FROG uses a phase retrieval algorithm to simultaneously gain information about the phase and amplitude. Using an optical gating to disperse the SHG signal, the detector not only record a function of intensity vs the temporal delay, but also a function of frequency. As shown in Figure 2.10, the horizontal axis is the temporal delay, frequency is on the vertical axis, and the third dimension is the intensity of the signal with a pseudo-color coding: purple indicates high intensity, and red indicates low. Both the phase and intensity of the pulse, as well as the pulse duration time, can be determined from this method. Due to the advantage of FROG over the simple autocorrelation, it is now more commonly employed in the research and industrial labs around the world. 


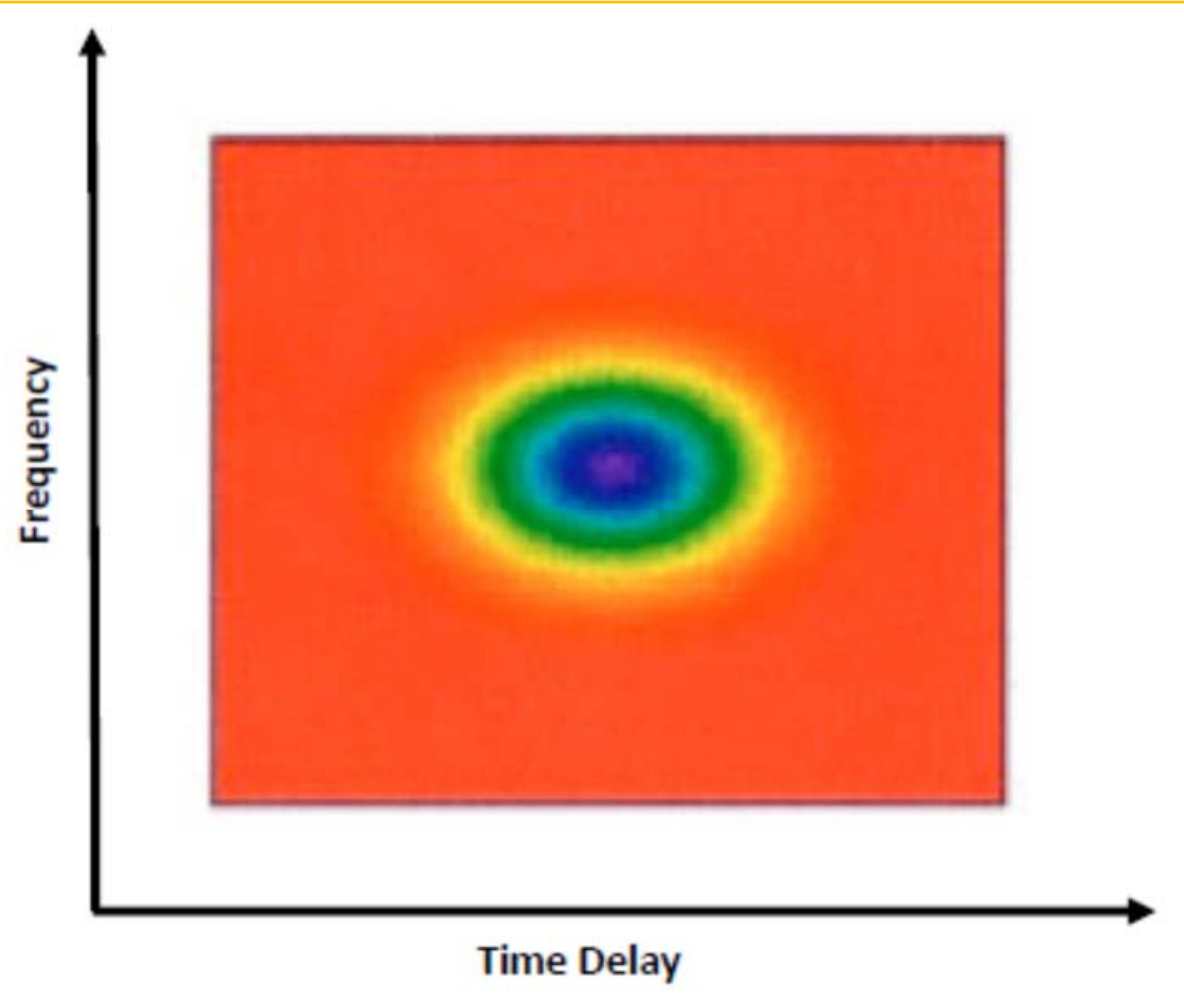

Figure 2.10. Second Harmonic Generation FROG Trace for a Negatively Chirped Pulse. Reprinted with permission from reference 187. Copyright 1997 AIP Publishing.

Other pulse characterization methods such as Grating-eliminated no-nonsense observation of ultrafast incident laser light e-fields (GRENOUILLE) [188], which is a simplified version of SHG FROG, and Spectral Phase Interferometry for Direct Electricfield Reconstruction (SPIDER) [189], are not described here because they are beyond the scope of the present work.

\subsection{Pump-Probe Detection Scheme}

The pulse duration time of a femtosecond laser is much shorter than the instrumental response time of any electronic devices, which means the transient signal cannot be measured directly on the femtosecond scale. Thus, several pump-probe detection schemes 
were developed in order to bypass this problem. Such techniques include Time-resolved Mass Spectroscopy [190], Time Gated Fluorescence Detection by Up-conversion [191], and Pump-Probe Transient Absorption Spectroscopy (TAPPS) [163]. TAPPS is the technique used in the present work.

Similar to intensity autocorrelation, in the experimental setup of pump-probe detection scheme, the pump beam and the probe beam go through different beam paths and each path involves a retro-reflector. The retro-reflector for the probe beam is on a delay stage mounted to a rail track and can be adjusted manually. The retro-reflector of the pump beam is fixed on a motorized translation stage which can be moved alongside the beam path by a computer. Therefore the temporal delay time (which is in the femtosecond scale) between the pump and probe can be calculated from the difference in the length of the two beam paths (in the $\mu \mathrm{m}$ scale) which in turn is determined by a computer software.

Two pump-probe detection schemes are shown in Figure 2.11. The green arrow indicates the pump beam (photon energy $=532 \mathrm{~nm}$, pulse duration $=30 \mathrm{fs}$ ). When the pump is focused through the sample cell, the system being studied gets excited by the pump photon, and this event sets the experimental time zero. The delayed probe light is then used to monitor the state of the system after excitation. If the probe source is a monochromatic light (red arrow in Figure 2.11.a), the transient absorption (TA) signal is measured by a photodiode. If a supercontinuum white light probe source (rainbow arrow Figure 2.11.b) is used, the TA signal is measured by an array detector. 
a)

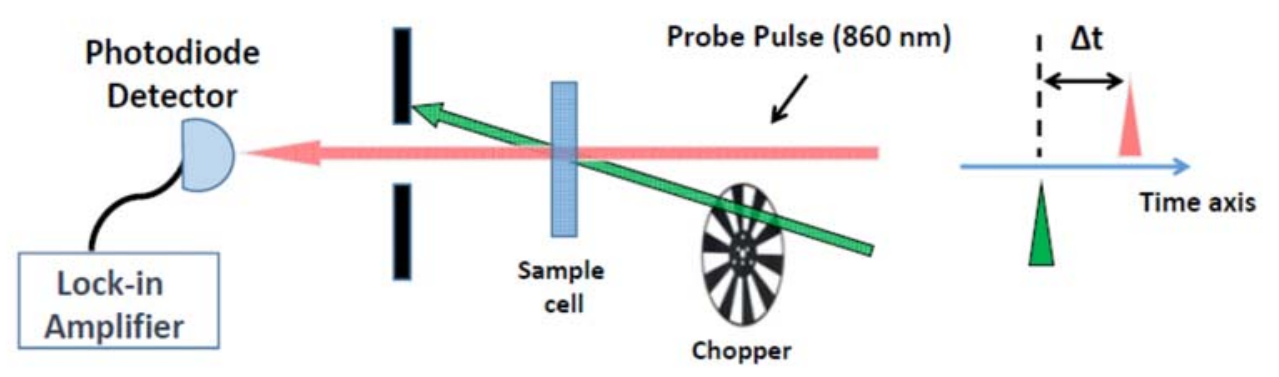

b)

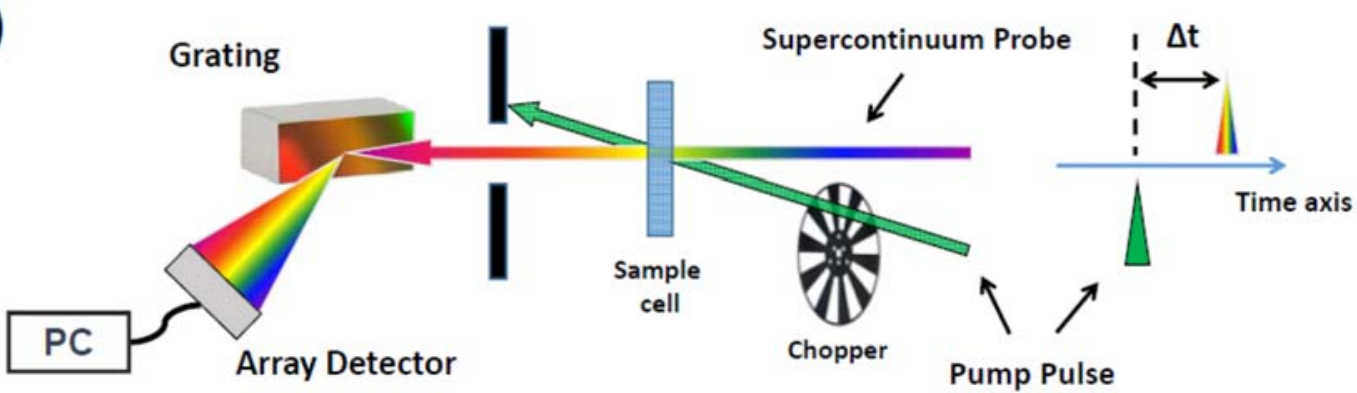

Figure 2.11. Pump-probe Detection Schemes with Two Different Probe Source

Pump pulse $(530 \mathrm{~nm})$ is illustrated by a green arrow. Probe pules is introduced with a temporal delay $\Delta \mathrm{t}$ and is shown as (a) a red arrow (860 $\mathrm{nm}$ monochromatic light) or (b) a rainbow arrow (supercontinuum white light).

Since the delay time between pump and probe is introduced by the delay stage, the temporal resolution of this pump-probe technique is only limited by the duration time of the original light source, and does not rely on the electronic response time of the detector. The delay time between the pump pulse and the probe pulse is calculated and recorded as $\Delta \mathbf{t}$.

An optical chopper revolving at $500 \mathrm{~Hz}$, which is half of the repetition rate of the laser source $(1 \mathrm{kHz})$, is used to modulate the pump beam so that half of the pump pulses are blocked. When the pump beam is blocked, only the probe beam passes through the sample, and the transmitted signal is used as the reference signal $\left(\mathbf{I}_{\mathbf{0}}\right)$. With the pump pulse blocked 
and unblocked, the changes in the optical density $(\Delta \mathbf{O D})$ are measured and recorded as a function of $\Delta \mathbf{t}$.

$$
\Delta O D(\Delta t)=-\log \left(\frac{I^{*}(\Delta t)}{I_{0}}\right)
$$

Where $I^{*}$ is the intensity of the transmitted probe light with a preceding pump pulse, and $\boldsymbol{I}_{\mathbf{0}}$ is the intensity of the transmitted probe light without the pump pulse (i.e., the system did not get excited). Based on the Beer's law, the transient absorption of the probe light is related to the population in the ground state and the excited states of the system. Therefore different TA signals can be obtained and used to study the system under excitation. Groundstate bleaching, excited states absorption, and stimulated emission, are the most common TA signals.

After the system got excited from the ground state $\left(\mathbf{S}_{\mathbf{0}}\right)$ to a higher electronic state $\left(\mathbf{S}_{\mathbf{1}}\right)$ by a pump beam (e.g., photon energy $=530 \mathrm{~nm}$, green arrow in Figure 2.12), the ground state is depopulated and the ground-state absorption decreases. Hence, $\boldsymbol{I}^{*}$ is larger than $\boldsymbol{I}_{\mathbf{0}}$, resulting in a negative $\Delta \mathbf{O D}$ signal, which is named the ground-state bleaching signal (light green arrow in Figure 2.12).

Meanwhile, electrons that are populating the excited state lead to an excited-state absorption. In this case, $\boldsymbol{I}^{*}$ is smaller than $\boldsymbol{I}_{\mathbf{0}}$, resulting a positive $\Delta \mathbf{O D}$ signal (blue arrows). While electrons relaxing back to the ground state via fluorescence or stimulated emission, another negative $\triangle$ OD signal will be observed (red arrow), and is called the stimulated emission signal. Due to the law of the energy conservation, the photon energy released by the stimulated emission is always smaller than the energy provide by the pump. 


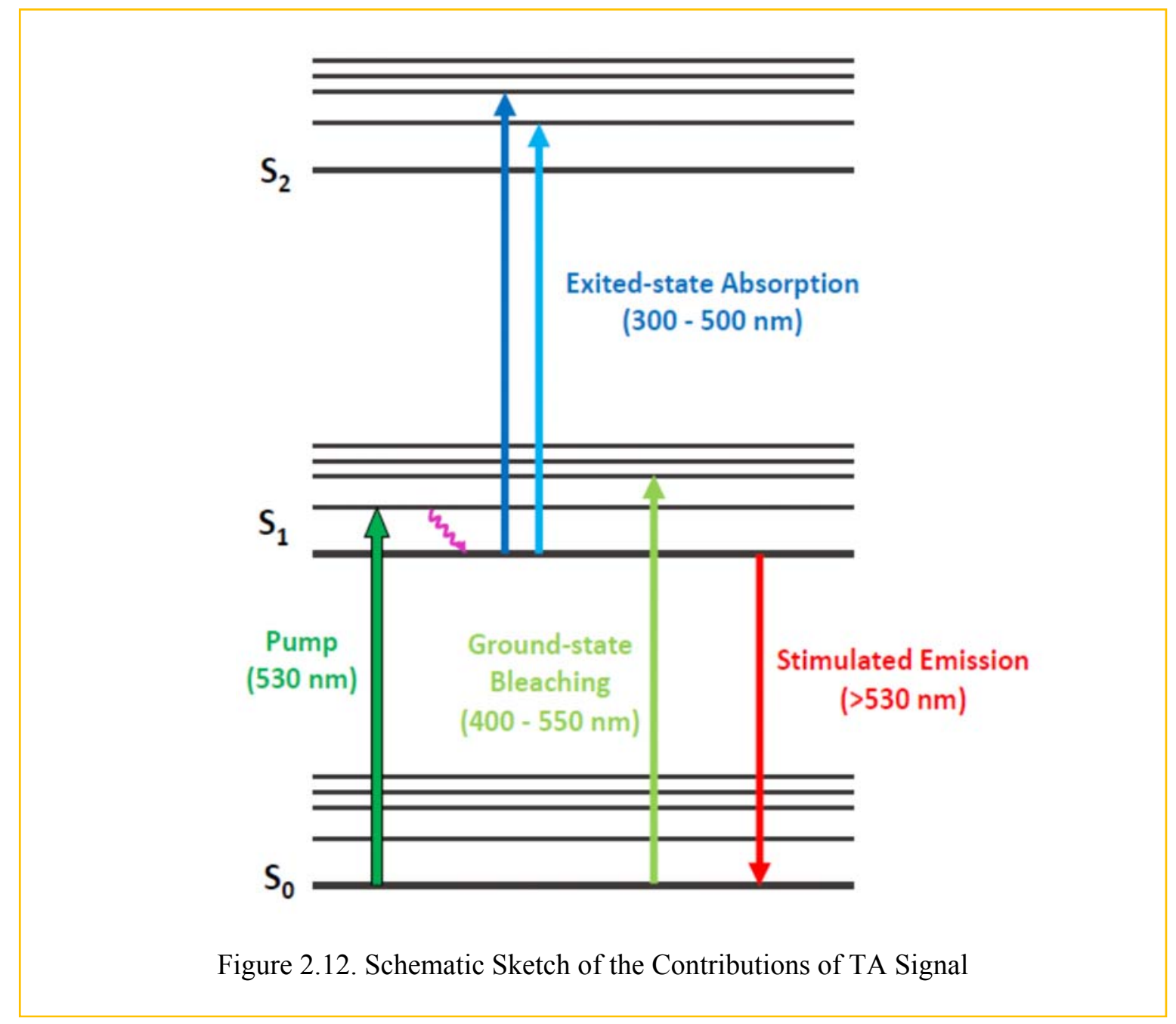

Sometimes, the TA signal is very comprehensive and consists of all the contributors mentioned above, and several other processes. Therefore, multi-peak fitting using Origin, and globe fitting using Mathcad, are required to analyze the comprehensive TA spectra (Section 2.7).

\subsection{Experimental Setups of Femtosecond Laser System}

The ultrafast spectroscopy in the present work was carried out in the Ultrafast Laser Facility inside the Conn Center for Renewable Energy Research in University of Louisville. 
The research focus of this facility is to characterize solar cell materials and investigate the fundamental dynamic processes, both in the materials and across the interfaces. The studies are important to the development of the photovoltaic devices, and have real life impacts in the field of renewable energy studies.

To properly maintain the stability and life time of the laser system, the facility is located in a humidity/temperature-controlled room with restricted access. Liebert precision cooling system is used to maintain the room temperature within the range of $23 \pm 1{ }^{\circ} \mathrm{C}$, and keep the humidity as $40 \%$ or lower. The Clark-MXR ultrashort pulse laser system was put on a stiffness-enhanced broadband-damped optical table supported by six pneumatic legs in order to isolate the vibrations. The table has all-steel isothermal construction and a precisionmachined matte-finish surface.

There are five main component boxes in the femtosecond laser system: one CPA laser head, two NOPAs, one SHG for NOPA 1, and one transient absorption pump-probe spectrometer (TAPPS).

As shown in Figure 2.13, CPA laser head generates a fundamental output (Red arrow: $775 \mathrm{~nm}, 1 \mathrm{kHz}$ repetition rate). The output is split and fed into the NOPAs, separately. In the NOPAs, the fundamental goes through two stages of parametric amplification and a pulse compressor.

Output of the NOPAs can either be used as a pump source (Green arrow: 450-700 $\mathrm{nm}$ ), or a probe source (Rosewood arrow: $800-1600 \mathrm{~nm}$ ), or an input for the SHG for ultraviolet pulse generations (Purple arrow: 266-350 nm). The supercontinuum white light (Rainbow arrow: broadband) is obtained by pumping a white light generation crystal with 
the CPA fundamental. Part of the fundamental is also fed into a SHG BBO crystal for frequency-doubling process (Blue arrow $388 \mathrm{~nm}$ ).

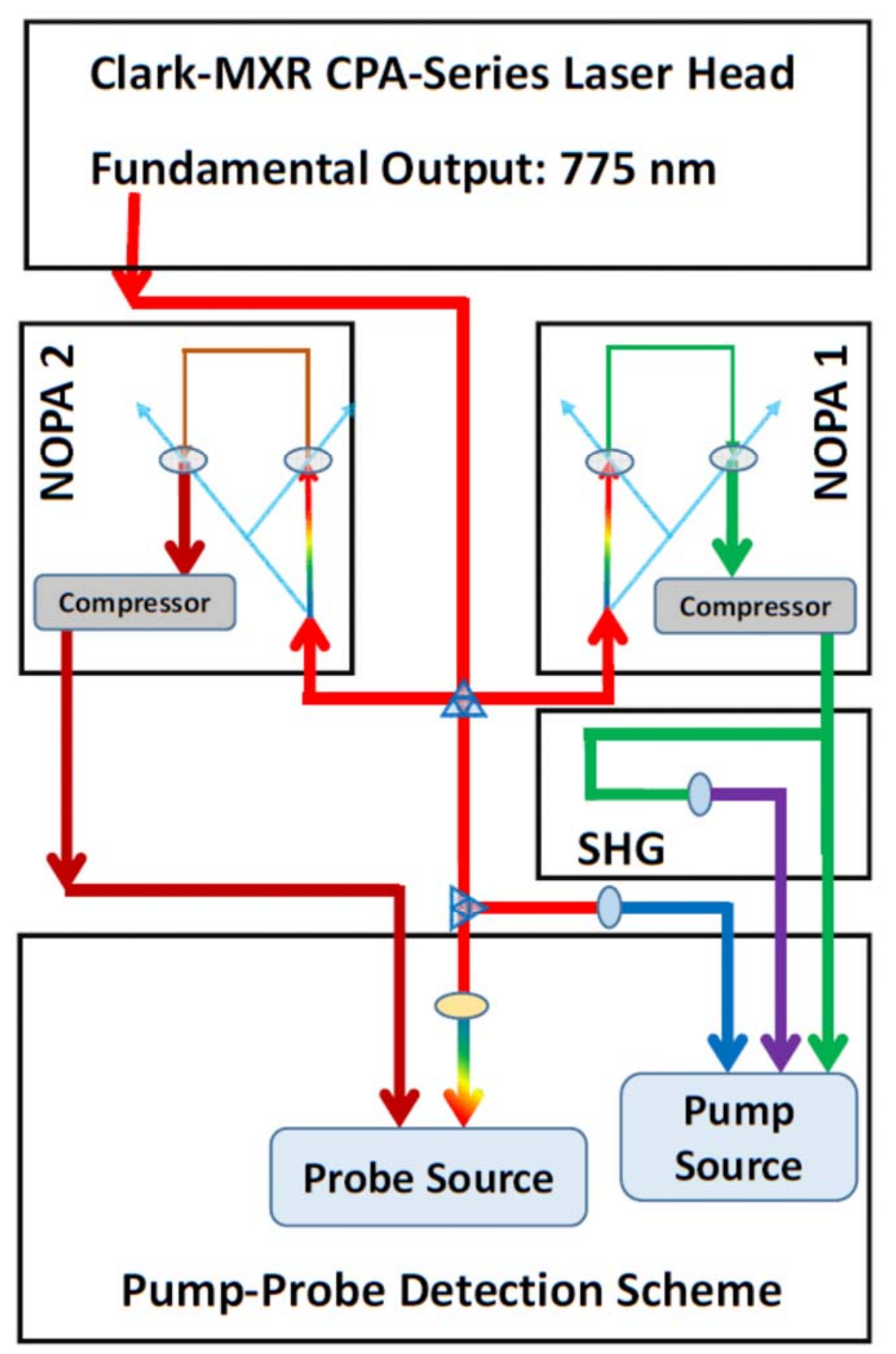

Figure 2.13. The Layout of the Femtosecond Laser Systems on the Optical Table 


\subsubsection{CPA Laser Head}

The CPA laser head is placed on the far end of the optical table. Utilities and accessories for the laser head, which include one power supply, two water-cooling systems, one nitrogen tank, one emergency power supply (EMS), one analog oscilloscope, and one operator interface, are all placed next to the table. The operator interface is a touch-screen computer with multi-tab functions that is used to control and monitor the CPA laser head (detail about the operator interface can be found in the manufacture manual [174]).

To protect the laser system, dry nitrogen is fed into the laser head constantly. The nitrogen is dried with gas drying unit filled with indicating DRIERITE. Indicating DRIERITE is a blue desiccant when they are dry, and changes to pink upon absorption of moisture. After the DRIERITE desiccants became completely exhausted (all pink), they should be baked by spreading them on a pan in one-granule-deep layers and placed in an oven at $210{ }^{\circ} \mathrm{C}$ for 1 hour, to achieve full dehydration [192].

There are two separate water-cooling systems, the internal and the external. The internal water-cooling system, controlled by a constantly running ThermoTek chiller (Model \#: T255P), is used for the thermal stabilization of the diode laser and the SErF fiber laser that inside the CPA laser head. The temperature set-point of the chiller is $19.30{ }^{\circ} \mathrm{C}$, and the chiller is filled with double de-ionized water. Since the diode laser and the SErF fiber laser are always on, the internal water cooling system is constantly running, and is powered by a EMS in case of a power outage.

The external water-cooling system is used to cool the flash lamp of the Nd:YAG laser inside the CPA laser head. Double de-ionized water drawn from a five-gallon water tank is used for the system cooling. The water tank is made by Lee Laser, Inc. (Model \#: 
010751-CLK3-001), and contains a stainless steel heat exchange coil. Lytron water chiller (Model \#: RC045J03BG2M007) is used to circulate de-ionized water inside the heat exchanger coil. At the beginning of a work day, the Lytron chiller needs to be turned on first, and the temperature of the chiller is set at $19{ }^{\circ} \mathrm{C}$ with a circulating pressure of $35 \mathrm{psi}$. Then the laser head is turn on by pressing the "Start System" button on the control panel. Once the system is ready for operating, system status on the control panel will change from "Warm up" to "On". Usually it takes at least 45 minutes to warm up the system before any experiments or laser tuning protocols can be performed [174].

When the flash lamp is on, and the temperature of the Lytron chiller is set at $19^{\circ} \mathrm{C}$, the water temperature inside the five-gallon water cooler is stabilized at $27 \pm 0.5^{\circ} \mathrm{C}$. This temperature is monitored by a VWR Traceable Digital Thermometer, and is kept under 32 ${ }^{\circ} \mathrm{C}$, otherwise, the flash lamp inside the laser head will overheat and the life time of the orings inside the flash lamp will shorten.

At the end of the work day, the system needs to be "shut down" on the control panel before turning off the external water-cooling system. The operator interface, the CPA laser head main power switch, and the internal water-cooling system should be left on. 


\subsubsection{Pump Pulse Generation: NOPA and SHG}

To setup the pump-probe detection scheme for obtaining TA spectra, a pump source and a probe source must be provided. Typically, the output of NOPA1 (green arrow in Figure 2.13) is used as the pump source for TAPPS. SHG of the fundamental (blue arrow), or SHG of the NOPA1 (purple arrow) can also be used as the pump source.

The principal behind NOPA and SHG is described in Section 2.2.2. Experimentally, the fundamental output of CPA is fed into NOPA1, and split by a beam splitter. One part of the fundamental outputs is fed into the SHG crystal to generate UV pulse (388 $\mathrm{nm}$ ), which is used as the pump source for the first stage and the second stage of NOPA1. The other part of the fundamental is power-modulated by a neutral density filter and size-modulated by an iris, and then focused through a white light generation crystal in order to provide supercontinuum seed source for the first stage of the NOPA1. In NOPA process, a high intensity pump beam at frequency $\boldsymbol{\omega}_{\mathrm{P}}$ is used to amplify a low intensity seed beam at frequency $\boldsymbol{\omega}_{\text {s. }}$ Phase matching condition and energy conservation law applies (Figure 2.6).

For maximum amplification, both pump and seed beam need to be overlapped inside the BBO crystal. The BBO crystal is cut at a $40.5^{\circ}$ angle and the thickness of the crystal is $0.1 \mathrm{~mm}$. Both crystal angle and pump delay time can be precisely tuned by micrometers, which allows precise values to be recorded after each optimization so that a good starting point is known for future tunings.

To monitor the NOPA amplification, Ocean Optics USB 4000 Fiber Optic Spectrometer is used to measure the photon energy of the amplified signal, and Ophir Nova II power meter equipped with 3A-FS thermal sensor is used to measure the pulse energy of the amplified signal. 
After the desired wavelength and the maximum power are acquired, the output of the first stage of NOPA1 is used as the seed source for the second stage amplification. The same optimizing procedure is repeated, and the amplified signal is compressed by a pair of grating mirrors. All crystal angle and pump delay time are recorded for future reference (Table 2.1).

Table 2.1. Micrometer Readings of Delay Time and Crystal Angle in NOPA 1 and 2

\begin{tabular}{|c|c|c|c|c|}
\hline $\begin{array}{c}\text { Micrometer } \\
\text { Readings on } \\
08 / 15 / 2016\end{array}$ & \multicolumn{2}{|c|}{ First stage amplification } & \multicolumn{2}{c|}{ First stage amplification } \\
\cline { 2 - 5 } & Delay Time & Crystal Angle & Delay Time & Crystal Angle \\
\hline NOPA 1 & $27.145 \mathrm{~mm}$ & $24.125 \mathrm{rad}$ & $15.65 \mathrm{~mm}$ & $16.175 \mathrm{rad}$ \\
\hline NOPA 2 & $31.5 \mathrm{~mm}$ & $0.125 \mathrm{rad}$ & $11.3 \mathrm{~mm}$ & $5.05 \mathrm{rad}$ \\
\hline
\end{tabular}

Generally, the output of the second stage amplification has an optimized power of 6 $\mathrm{mW}$ at $1 \mathrm{kHz}$ repetition rate, and a frequency tuning range of $450 \mathrm{~nm}-700 \mathrm{~nm}$. The pulse duration time is $30 \mathrm{fs}$ after the pulse compression. (Detailed schematic diagram and tuning procedures of NOPA can be found in the manufacture manuals [193])

NOPA1-SHG is a small accessory optical box for the use of expanding the tuning range of NOPA1 into UV frequency. The output of NOPA1 can simply pass through the SHG box, or undergo the SHG process by changing the beam path with the help of two flipping mirrors. The BBO crystal for NOPA1-SHG process is mounted on a true gimbal mount with full angular control. 
When the photon energy of the incoming beam is $2.33 \mathrm{eV}(532 \mathrm{~nm})$, and the SHG crystal is properly positioned, the photon energy of the output beam should equal to $4.66 \mathrm{eV}$ (266 nm), i.e., frequency doubling is achieved.

The BBO crystal for SHG is cut with a $30^{\circ}$ angle and the thickness of the crystal is $2 \mathrm{~mm}$. The same BBO crystal is employed for the fundamental-SHG stage which is located outside the NOPA1-SHG box (Figure 2.13). In the fundamental-SHG process, a $388 \mathrm{~nm}$ $(3.20 \mathrm{eV})$ pulse is generated from the $775 \mathrm{~nm}(1.60 \mathrm{eV})$ fundamentals.

\subsubsection{Probe Pulse Generation: Supercontinuum White Light}

While NOPA1 provides the pump source in the range of $450-700 \mathrm{~nm}$ for the TAPPS, NOPA2 is usually used to provide a monochromatic probe source in the range of $800-1600 \mathrm{~nm}$ (Figure 2.13). A probe source with a near IR detection range is very useful in the optical characterizations of the semiconductor films and nanoparticles, as well as in the studies of pharmaceutical, agricultural, and atmospheric chemistry.

NOPA1 and NOPA2 share the same basic concepts, optics layouts, and tuning procedures. However, they have very different value in pump delay time and the crystal angle. Table 2.1 listed the readings of the micrometers from the first and second stage of NOPA1 and NOPA2, respectively.

With the same frequency $\boldsymbol{\omega}_{\mathrm{P}}=3.20 \mathrm{eV}(388 \mathrm{~nm})$ is used to pump the BBO crystal, the frequency of the desired NOPA2 output is $1.44 \mathrm{eV}(860 \mathrm{~nm})$, and the frequency of the idler is $1.76 \mathrm{eV}(705 \mathrm{~nm})$. While in NOPA1, the frequency of the signal is $2.33 \mathrm{eV}$ (523 $\mathrm{nm})$, and of the idler is $0.87 \mathrm{eV}$. 
The output of NOPA2 is guided into the TAPPS box and used as the monochromatic probe source. The monochromatic probe goes through a retro-reflector which is fixed on the probe delay rail that is used for adjusting the probe delay time. Then the probe beam is focused through the sample cell and the transmitted signal is monitored by a photodiode detector (Thorlabs InGaAs Biased Detector, Model \#: DET10C, Detection Range: 700 $1800 \mathrm{~nm})$.

When the monochromatic probe source is used, the data acquisition is accomplished with the help of Stanford Research Systems digital signal processing (DSP) Lock-in Amplifier (Model \# SR810). A Lock-in Amplifier is a signal processor that extracts signal with a known carrier wave from a noisy background. First, the lock-in amplifier takes the input signal, multiples it by the reference signal, and integrates it over a specific time. The resulting signal is a DC signal, where the contribution from any signal that is not at the same frequency (i.e., noise) is attenuated to almost zero. Any out-of-phase components of the signal are also attenuated, so that the signal to noise ratio is significantly increased by this process. The setting of the lock-in amplifier for data acquisition can be found in our group wiki page [194] (Figure 2.14). The time constant is set as $100 \mathrm{~ms}$, the sensitivity is $20 \mathrm{mV}$, and the trigger reference signal is a sine wave function.

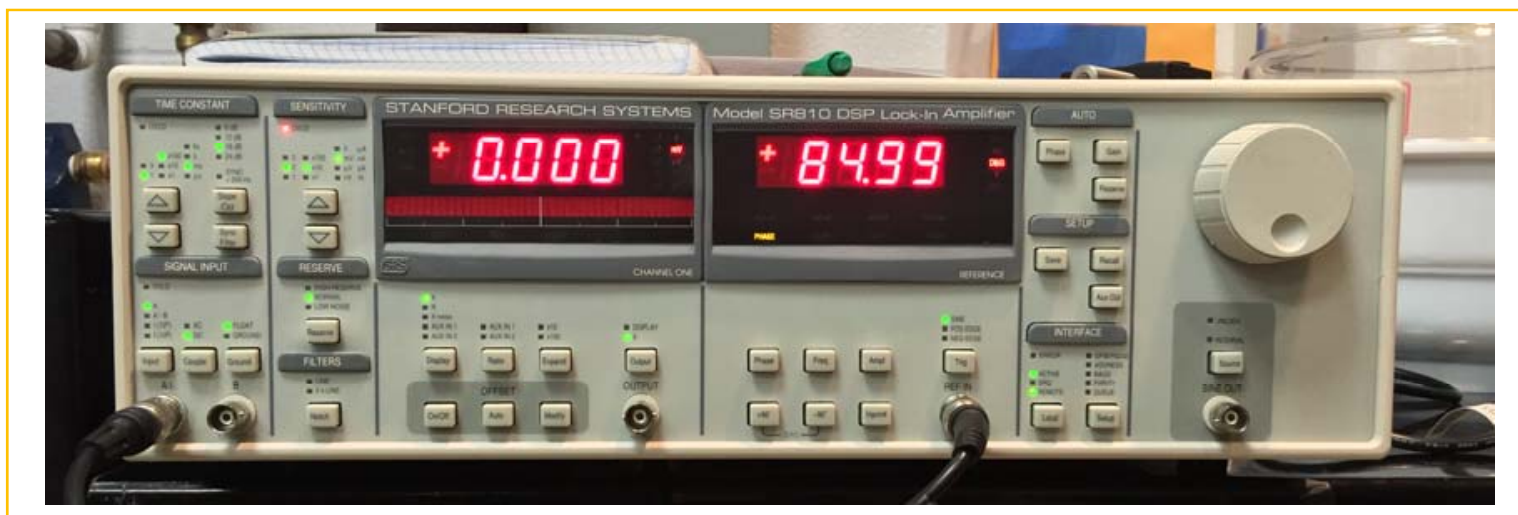

Figure 2.14. Stanford Research System Lock-in Amplifier 
Supercontinuum white light is more commonly used as the probe source for TA experiments. It provides a broadband detection of the TA spectra that contain comprehensive information.

Figure 2.15.a illustrates the generation and detection of the Supercontinuum white light. First, the fundamental $(776 \mathrm{~nm})$ enters the TAPPS box, and goes through a retroreflector that can manually slide along the white light delay rail, so that the delay time of the white light can be adjusted. Then the fundamental is focused into the white light generation crystal. After the supercontinuum generation, another focus lens is used to collimate the diverging beam which is caused by the self-focusing process happening inside the crystal. The fundamental is blocked by a short pass filter, and the pulse energy of the white light is modulated by a neutral density filter.

The white light probe then passes through the sample cell, and is guided into the detection box. A curve grating mirror in the detection box disperses the transmitted white light onto an array detector.

Before running the TA experiments, the white light probe needs to be optimized by adjusting the focus lens and the neutral density filter, in order to reach an optimized intensity, a good spectral coverage, and a low noise level. The intensity and the quality of the white light signal can be monitored in the TAPPS software. The initial intensity of the signal (without sample cell) should be around 55000 counts but does not exceed the detection limit which is 60000 counts [195]. The transmitted signal (with sample inside sample cell) should be around 20000 counts across the spectral range of $430-730 \mathrm{~nm}$ (Figure 2.15.b), so that the absorption of the sample is around 0.44 a.u. The noise level of the white light should be less than $2 \%$ ( 0.02 percentage RMS) relative to the signal level (Figure 2.15.c). 
a) White Light Generation and Detection Scheme

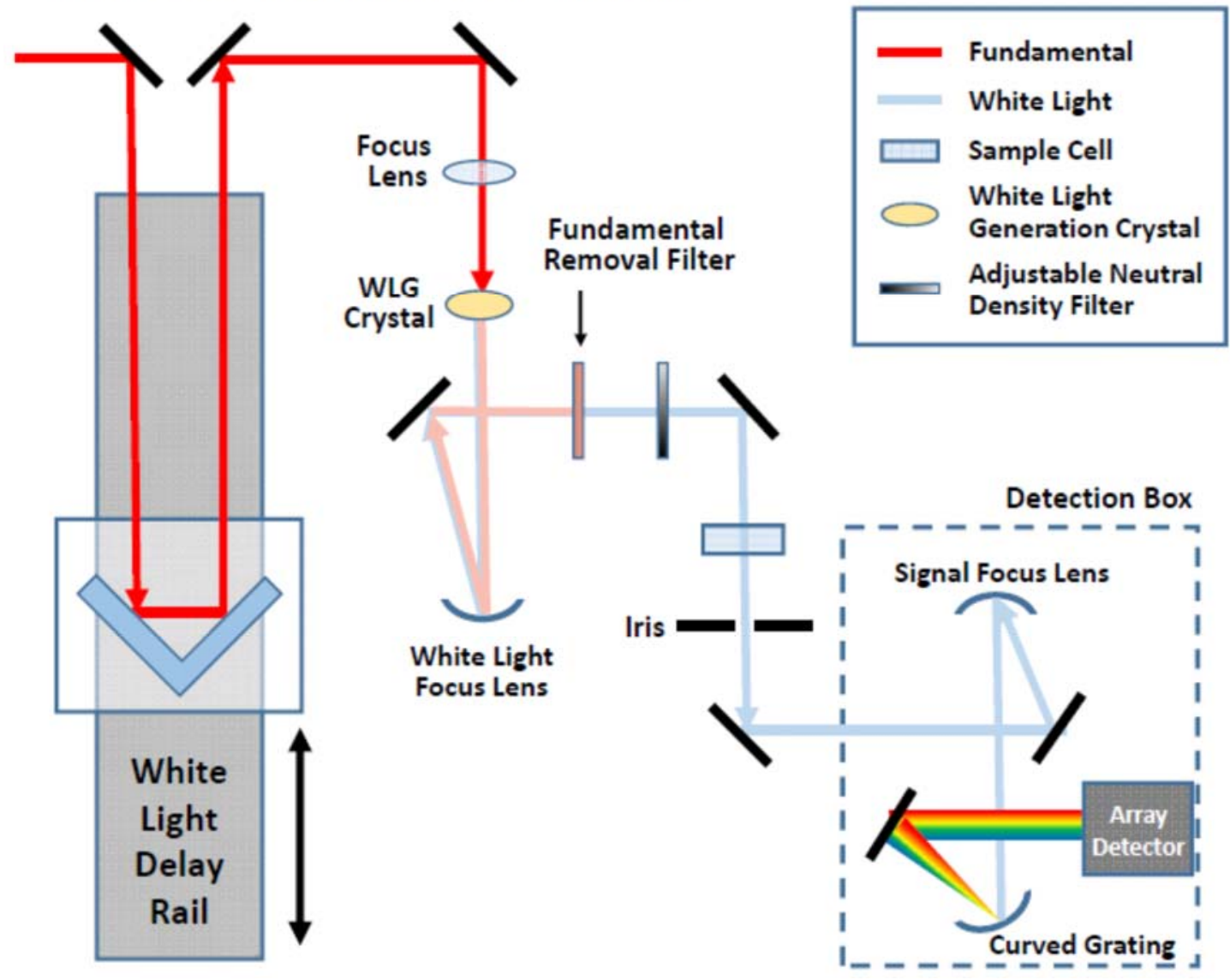

b) Intensity of White Light Signal (Unit: Counts)

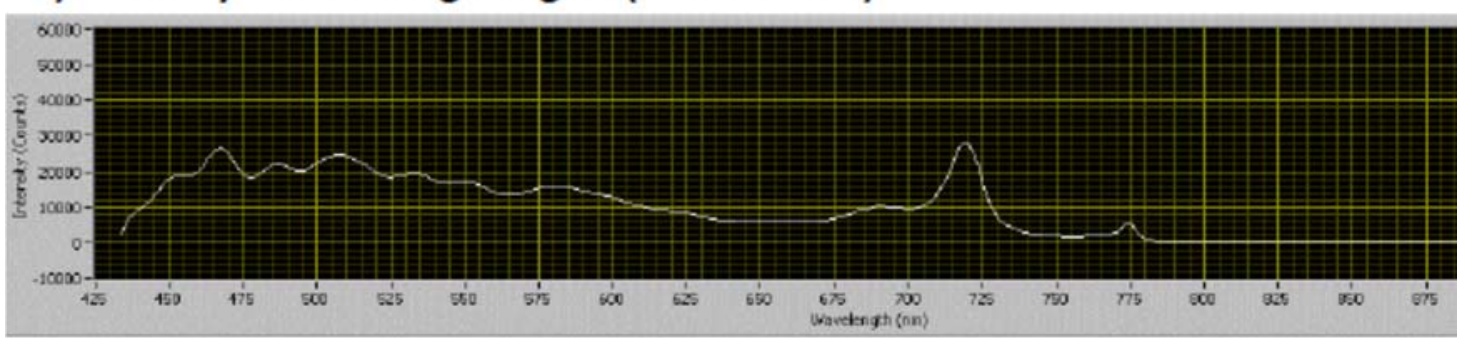

c) White Light Noise (Unit: percentage RMS "root mean square")

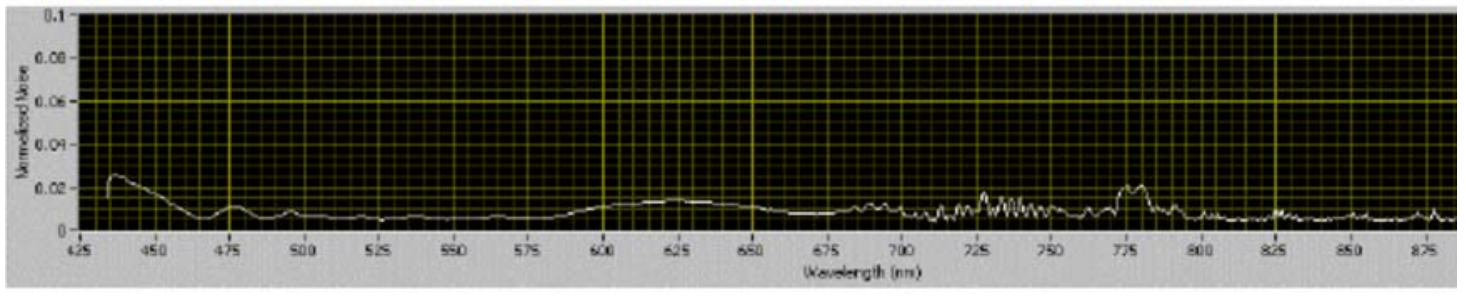

Figure 2.15. Supercontinuum White Light Generation and Detection 


\subsubsection{Transient Absorption Pump-Probe Spectroscopy (TAPPS)}

There are a total of three delay rails inside the TAPPS box: the pump delay rail, the white light delay rail, and the monochromatic probe light delay rail. The pump delay rail is the only one with a motorized translation stage controlled by computer. The motor has a total scan range of $24.2 \mathrm{~cm}$ (equal to $1.6 \mathrm{~ns}$ of delay time) with a resolution of $0.1 \mu \mathrm{m}$ (equal to 0.66 fs time resolution). After the delay stage, the pump beam is modulated by the ThorLabs optical chopper (Model \#: MC2000) which is revolving at $500 \mathrm{~Hz}$ (Figure 2.16). The chopper is connected via a BNC cable to the data acquisition card of the computer, and can be manually or electronically turned on.

Three types of pump sources and two types of probe sources (Figure 2.13) are introduced in earlier sections, which give a total of six pump/probe combinations: NOPA1/Whitelight， NOPA1/NOPA2， NOPA-SHG/Whitelight， NOPA-SHG/NOPA2, Fundamental-SHG/Whitelight, and Fundamental-SHG/NOPA2. For each pump/probe combinations, the proper time zero positions of the delay stages are calculated and corrected based on real test runs. Those positions are marked on the delay rail and logged into an excel table which can be found in our group wiki page [196].

Before the experiment, the probe delay stage is manually adjusted to its time zero position based on which pump/probe combination is currently employed, and the pump delay stage is moved electronically to the corresponding startup position by putting a time zero encoder into the TAPPS software.

The pump beam and the probe beam need to be optimized and overlapped inside the sample cell before the TA experiments. To overlap the pump and probe beam, a pin hole $(200 \mu \mathrm{m})$ is placed on an OptoSigma three-dimensional translation stage, replacing the 
sample cell. The translation stage is adjusted with three micrometers until probe beam passes through the center of the pin hole. Then the pump beam is adjusted to pass through the pin hole. After both pump and probe beams are focused through the pin hole, the sample cell is put back onto the translation stage replacing the pin hole. After the above procedure, the overlapping and focusing points of the pump and probe beam will reside precisely inside the sample cell.

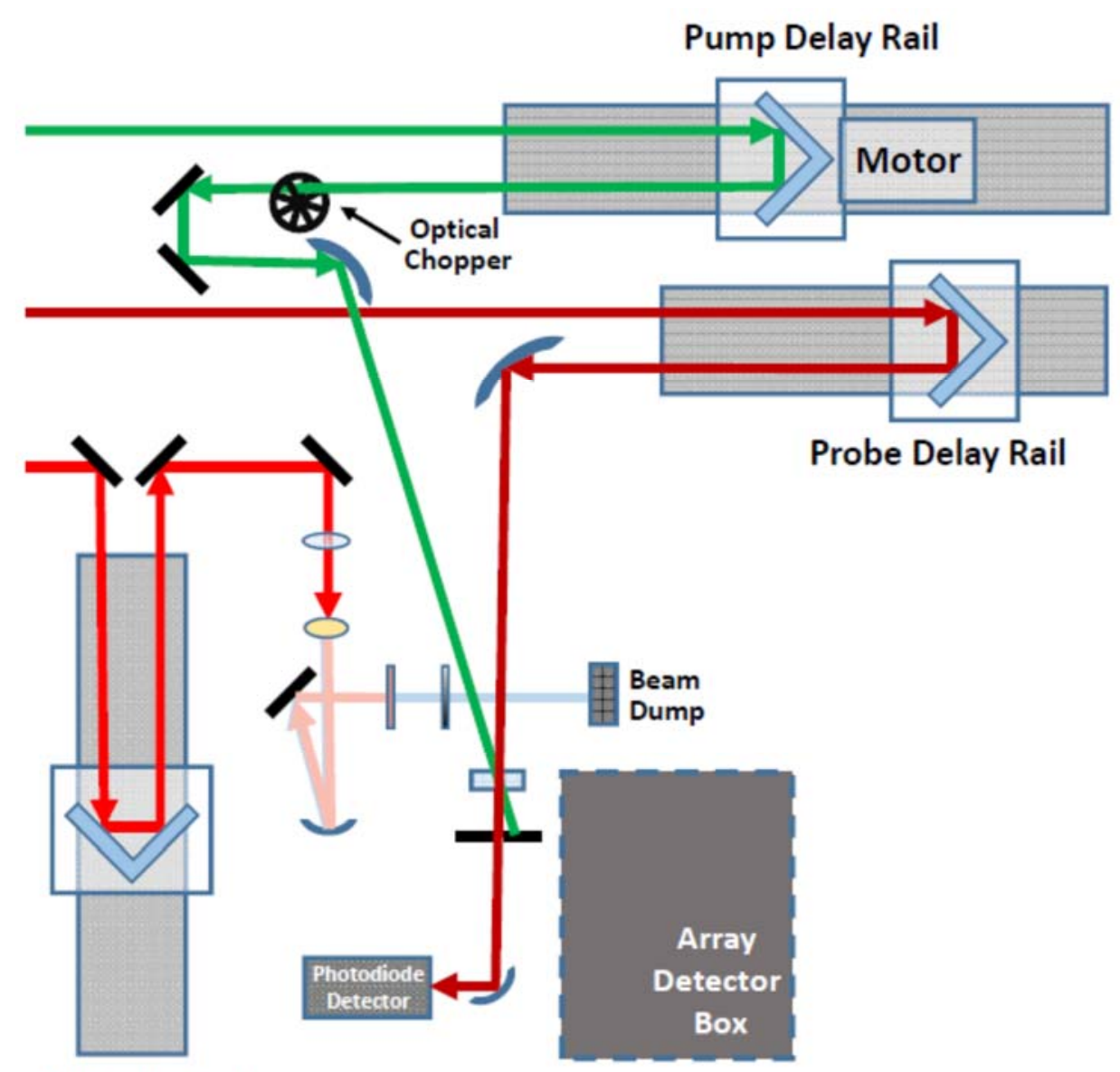

WL Delay Rail

Figure 2.16. Monochromatic Porbe Source Detection Schemes 


\subsection{Data Acquisition Software}

The software for the TAPPS is written in Labview 10 and compiled for use on the system. Custom programing can be done on the software if a copy of Labview 10 is available. TAPPS software provides a single interface that controls the delay stage, the probe detector, the chopper, as well as acquire, generate, save, and display the data.

The first main tab of the TAPPS software is the Scan Data Tab, which controls the starting, stopping and pausing scans. The name and the location of the data file can be set in the tab. The pump delay stage can be moved by changing the delay time. Increasing the delay time moves the stage platform away from the motor and vice versa. The Scan Data Tab has a sub tab which is the Scan Range Settings Tab. This sub tab allows the user to set the designed scan type: the single region linear scan, the multi region linear scan, and the quasi exponential scan.[195]

The most of exciting dynamics in the semiconductor nanoparticle materials are usually happening during the first few ps after the system is excited. Therefore a stepwise multi-region scanning method is often used, so that there are more data points acquired during the first $10 \mathrm{ps}$, but meanwhile the total amount of data points is not overwhelming which will crush the software. For example, when $\Delta \mathrm{t}=-2 \mathrm{ps} \sim 10 \mathrm{ps}$, the step size is set as $10 \mathrm{fs}$; when $\Delta \mathrm{t}=10 \mathrm{ps} \sim 100 \mathrm{ps}$, the step size is set as $2000 \mathrm{fs}$; when $\Delta \mathrm{t}=100 \mathrm{ps} \sim 1 \mathrm{~ns}$, the step size is set as $20000 \mathrm{fs}$.

The second main tab is the Detector Controls Tab, which contains the White Light Noise sub tab, the Free Running Delta OD sub tab, and the Spectra Calibration sub tab. The White Light Noise sub tab can display the intensity and the percentage RMS noise of the white light at each wavelength. During the white light probe optimization, the detector can 
be activated using the Start button in this sub tab. The white light intensity is used to align the spectrometer to make sure maximum optimized signal is incident on the detector. An intensity over 60000 counts indicates the detector is saturated. With the pump beam blocked, the RMS percentage noise should be less than $2 \%$ for good data acquisition. The Free Running Delta OD sub tab is used to measure the signal time TA spectra outside a scan for testing the pump-probe overlap. And the Spectra Calibration sub tab is used for the wavelength calibration.[195]

The third main tab is the Stage Control tab. All the stage parameters are displayed in this tab and are used to control the time zero position of the pump delay stage. The Chopper Control tab is the last main tab. It allows the software to control the chopper to be toggled on or off. After turning on the optical chopper, and making sure both detector and motorized stage are functioning properly, data acquisition process can be performed.

The above descriptions are for the multi-channel white light probe detection scheme. The TAPPS software for the single channel detection is a separate program from the multichannel software, but it is essentially structured the same, with only the Detector Control tab significantly changed since the detection is now done by the lock-in amplifier. First, the system collects the data at the first time point and stores them in the lock-in amplifier based on the Sampler Per Time Point. Then the average data is transferred from the lock-in to the computer before the stage moves to the next time point and starts the data collection process all over. The data vs time delay trace is displayed in the Scan Data main tab.[195]

The single channel detector software can also be used for autocorrelation methods. More details about TAPPS software can be found in the manufacture manuals [195]. 


\subsection{Post Data Acquisition Analysis}

\subsubsection{Group Velocity Dispersion (GVD) Correction}

When using supercontinuum white light as probe source, the array detector records the optical intensity at different wavelengths at each $\Delta \mathrm{t}$. Therefore, the retrieved data is exported as a three-dimension matrix containing both temporal and frequency domain information, and $\triangle \mathrm{OD}$ is on the third dimension axis (Figure 2.17). Due to the dispersion of the white light by the grating mirror, the raw 3D data needs to undergo GVD correction before further analysis.
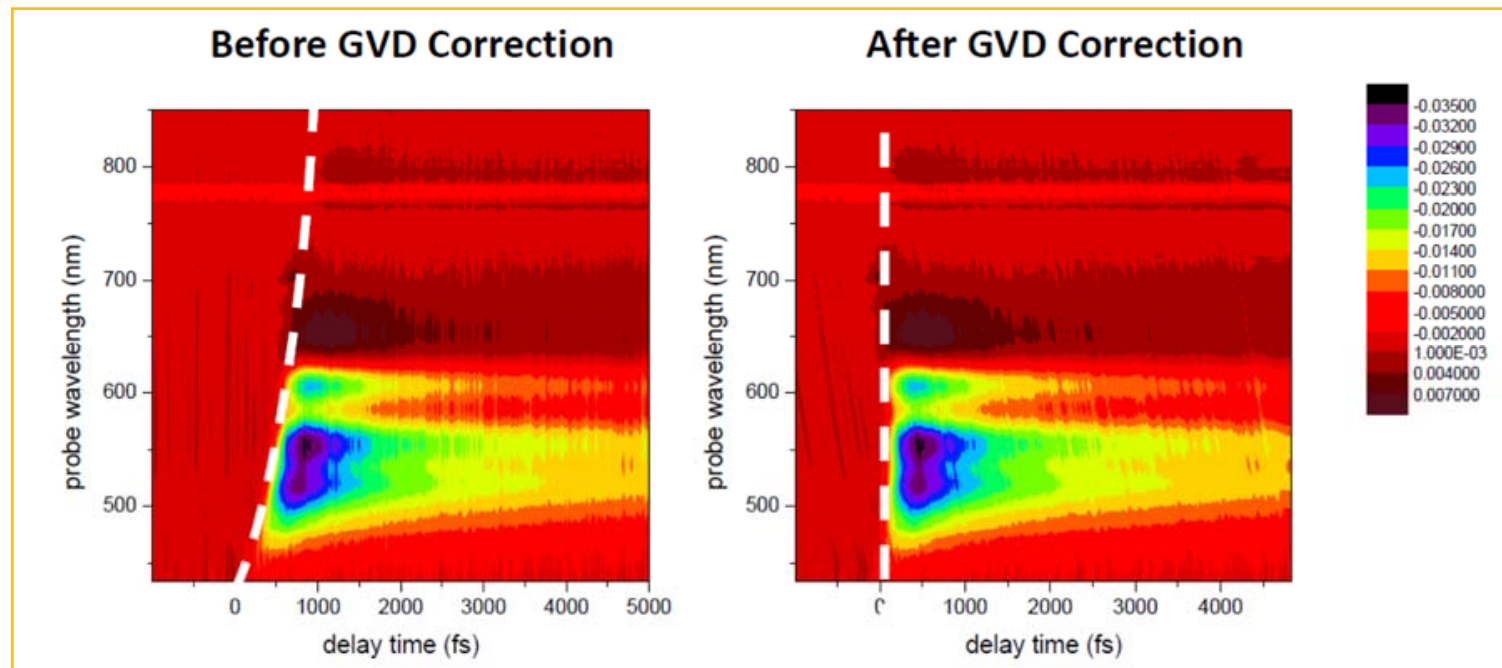

Figure 2.17. Group Velocity Dispersion (GVD) Correction of 3D Transient Absorption Spectra

When the transient signal goes into the detection box (Figure 2.15.a), the curved grating mirror disperses the white light onto the array detector. As the white light got dispersed, photons with different frequencies (photon energies) travel slightly different in distance (between the grating and the detector), which introduces false time zeroes across the whole wavelength domain. As shown in Figure 2.17.a, the white curved line indicates 
the shifting effect of time zeros. This false-time-zero line is retrieved (by choose twenty data points from the raw 3D data) and fitted with a power function in OriginPro 8.1 :

$$
\Delta t(\lambda)=\Delta t_{0}+A\left|\lambda-\lambda_{0}\right|^{p}
$$

Where $\lambda_{0}$ is a reference wavelength, and $\Delta t_{0}$ is the shifting of the time zero at $\lambda_{0}$. Using the parameters (such as $\mathbf{A}$, and $\mathbf{p}$ ) that determined from the power function fitting, the shifting of time zeros at all wavelength, $\Delta \mathbf{t}(\lambda)$, can be calculated. Then the equation and parameters are fed into a C-code executable program which preforms the GVD correction and generates an output file containing a GVD corrected 3D matrix (Figure 2.17.b). Both software codes of GVD program and detail fitting procedures can be found in Appendix IV.

\subsubsection{MathCAD Global Fittings}

The information retrieved from the wavelength domain is always related to the electron population on different energy levels based on Beer's Law, therefore can be simulated as a combining effect of several Gaussian line shape functions [31]. The rising curve and decay curve on the time domain can be fit as an exponential decay with one or more time components. Based on this concept, globe fitting and simulation can be carried out with the help of MathCAD or other data analyzing software (more details about global fitting can be found in Section 3.3.2, and the MathCAD coding can be found in Appendix II). 


\subsection{Ultrafast Lab Daily Maintenance}

In order to keep the laser running properly and to prolong the life time of the system, routine maintenance needs to be performed regularly. A lab log book needs to be filled-in everyday to track the performance of the CPA laser head, as well as the output power of the two NOPAs. Any performance errors of the temperature control system and the water cooling systems also need to be logged into the log book. For the external water-cooling loop, the de-ionized water in the Lytron Chiller needs to be changed every two months, the particle filter for the chiller should be changed every six month. The status of the internal water-cooling system needs to be monitored, and the double de-ionized water should be refilled when the water level is low (indicator shows "water level: wrong"). The Nd:YAG flash lamp needs to be replaced when the ORC current level reaches 29 amps on the "power supply" screen of the control panel. The Nd:YAG rod seals should be changed every 1500 operating hours. The status of the Liebert temperature controlling system also needs be checked regularly. The desiccants in the nitrogen drying unit need to be exchanged and baked when the color of the desiccants completely transfers from blue to pink. 


\section{CHAPTER 3}

\section{ENERGY LEVEL ALIGNMENT AND PHOTOINDUCED ULTRAFAST CHARGE TRANSFER PROCESSES OF CdSe NANOCLUSTER PASSIVATED WITH LIGAND PHENYLDITHIOCARBAMATE}

This is the first research project during my Ph.D. study. ${ }^{1}$ The motivation of this project is to study a promising electronic material for solid-state device fabrication: $1.6 \mathrm{~nm}$ diameter $\mathrm{CdSe}$ semiconductor nanoclusters ( $\mathrm{SCNCs})$. The charge generation and charge transfers from the inorganic CdSe nanocluster cores to the surrounding ligands have a strong influence on the device efficiency. In this context, we report the excited-state dynamics of the molecule-like $1.6 \mathrm{~nm}$ diameter $\mathrm{CdSe} \mathrm{SCNCs}$ that are passivated with a highly conjugated ligand: phenyldithiocarbamate (PDTC). Femtosecond transient absorption spectroscopy studies revealed a sub-picosecond hole transfer ( $\tau \approx 0.9 \mathrm{ps}$ ) from CdSe core to its PDTC shell. Strong electronic interaction, wave-function induced hole delocalization, and subpicosecond hot electron transfer $(\tau \approx 0.2 \mathrm{ps})$ from excited states of CdSe to the interfacial states, are observed. A series of control experiments were performed by varying the size of CdSe nanocluster cores (1.6 nm vs $2.9 \mathrm{~nm}$ ), the photon energy of the pump laser source (388 $\mathrm{nm}$ vs $490 \mathrm{~nm}$ ), and by addition of an electron quencher, benzoquinone (BQ), and a hole quencher, pyridine (Py). These control experiments ruled out alternative mechanisms and confirmed the critical role of energy level alignment between the CdSe nanocluster and

\footnotetext{
${ }^{1}$ This Chapter has been published in The Journal of Physical Chemistry C [31].
} 
PDTC ligands. Understanding the charge carrier dynamics across the CdSe NCs-organic ligands interface is essential to various physical phenomena, such as hot carrier relaxation, and multiple exciton generation, which together could aid in the design of high efficiency solar cells and photocatalysts.

\subsection{Introduction}

Efficient charge transfer (CT) across the semiconductor quantum dot (QD)-surface ligand interface is crucial in enhancing the performance of ligand-passivated QDs in many potential applications such as solar cells [197-202], light-emitting diodes [112, 116, 123, 203], and other optoelectronic devices [204-207]. Commonly used long alkyl chaincontaining surface-passivating ligands hinder $\mathrm{CT}$ processes. To improve such processes, various dye molecules were used to modify the QD's surface, and ultrafast spectroscopy techniques were implemented to study the electron transfer (ET) or hole transfer (HT) dynamics [208-210]. It was shown that both the ET and HT processes depend on the structural parameters of the QDs, such as size, composition, chemical nature of the surfacepassivating ligands, and electronic interaction between QDs and ligands [27, 50, 207, 211219]. Compared to the amount of information available on excited state dynamics of metal chalcogenide QDs, the scientific knowledge concerning ultrasmall semiconductor nanoclusters (SCNCs, $<2.0 \mathrm{~nm}$ in diameter) is very limited $[219,220]$ and mostly focused on theoretical calculation [51, 221-223]. To the best of our knowledge, no report is available studying the dynamics of both ET and HT across the ultrasmall SCNC-surface ligand interface. 
Ultrasmall SCNCs bridge the gap between small molecules and QDs with discrete highest occupied molecular orbital (HOMO) and lowest unoccupied molecular orbital (LUMO) energy levels [18, 62, 224]. In QDs a low percentage of atoms occupy the surface and the variety of surface facets leads to inhomogeneous composition, which results in precise information on the electronic structure and dynamics remaining elusive. In contrast, $1.6 \mathrm{~nm}$ diameter $\mathrm{Cd}_{34} \mathrm{Se}_{34} \mathrm{SCNCs}$, also known as "magic-size" clusters, contain nearly $80 \%$ surface atoms $[83,84,225]$, and thus their surface structure is expected to have profound effects on their photophysical properties. Atomically precise, ultrasmall SCNCs therefore have much better ability to provide quantitative information about ET and HT dynamics than traditionally studied QDs through appropriate selection of surface-passivating ligands, which induce a strong SCNC-ligand interaction. Recently, Weiss and co-workers [56, 57] and our collaborators, Teunis and Sardar [226], have shown that attaching phenyldithiocarbamate (PDTC) ligands to CdSe QDs, and SCNCs, respectively, reduced the optical bandgap due to expansion of the hole wave function outside of the inorganic core to the ligand monolayer. This expansion is from interfacial orbital mixing due to strong interaction between the SCNC and its ligands [227, 228]. Nearly a 2-fold higher reduction of the optical bandgap of CdSe SCNCs is observed in comparison to QDs, presumably due to their very small size. 


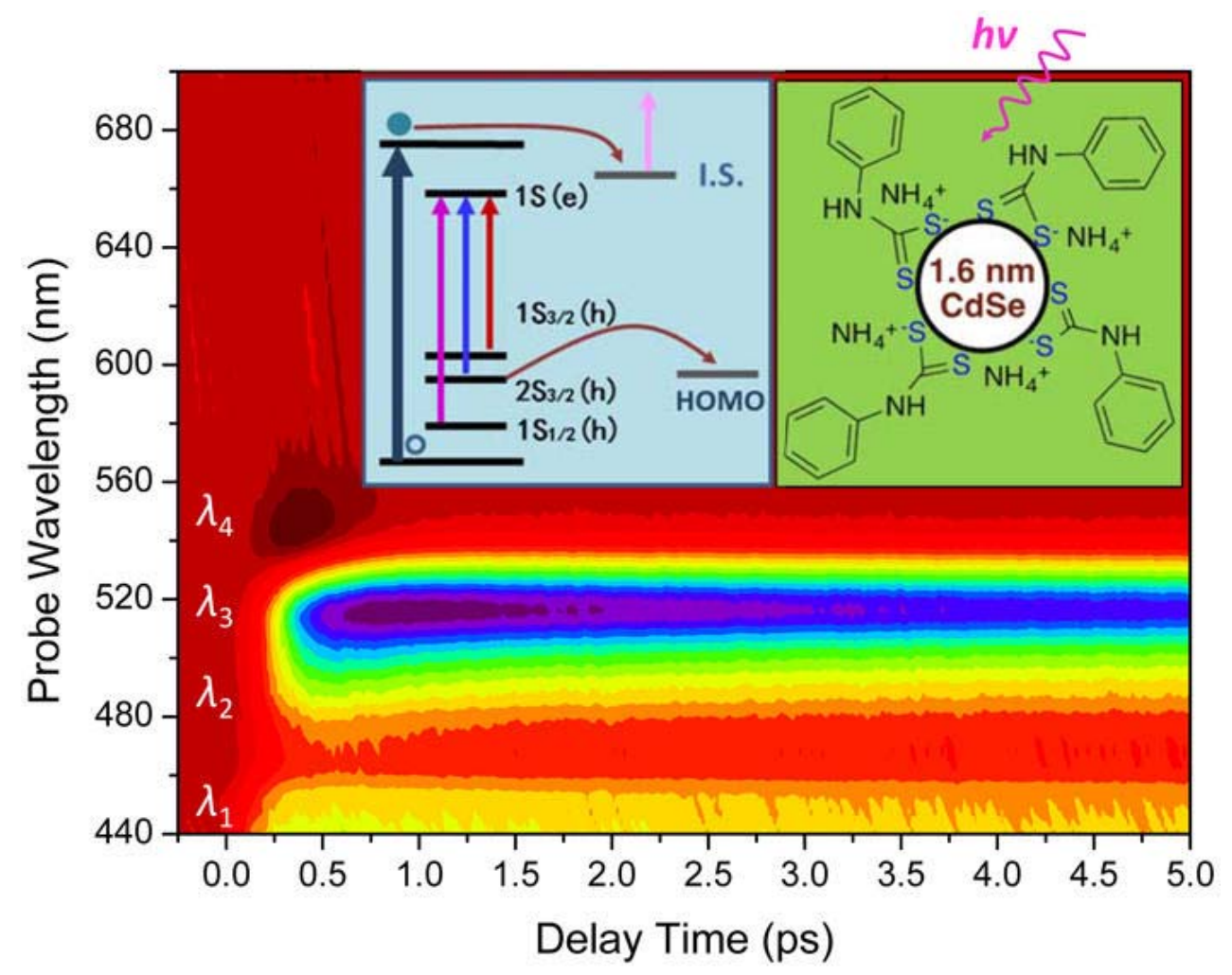

Figure 3.1. Three-dimension Transient Absorption Spectrum of $1.6 \mathrm{~nm} \mathrm{CdSe}$ Semiconductor Nanoclusters Passivated with Phenyldithiocarbamate

Delay time (picoseconds) at $\mathrm{x}$-axis, probe wavelength (nanometer) at $\mathrm{y}$ axis, and the change in optical density $(\Delta \mathrm{OD})$ at the third dimension. CdSe nanocluster passivated with ligand is shown in the right (green) inset, and the proposed energy transfer diagram is shown in the left (blue) inset.

In this context, we report for the first time the study of excited state dynamics of PDTC-passivated $1.6 \mathrm{~nm}$ diameter CdSe SCNCs conjugates. We observed a sub-picosecond time scale for both ET and HT processes when the SCNCs were excited at a photon energy much larger than the optical bandgap. We explored the dynamics by selectively changing the excitation energy and nanocluster size to prove that interfacial orbital mixing and formation of interfacial states promoted the hole transfer and hot electron transfer from the SCNC core to the PDTC ligand monolayer. Furthermore, appropriate energetic alignment 
between SCNC orbitals and PDTC molecular orbitals (MOs) is critical to the observed ultrafast ET and HT processes. Understanding such excited-state dynamics could open up new avenues for the design of new ligands as well as new SCNC-ligand conjugates that will exhibit fast charge separation and slow recombination, which have great potential to increase the efficiency of solar cells prepared from them.

\subsection{Experimental Details}

\subsubsection{Synthesis of Ligand-Passivated SCNCs}

Materials. Cadmium chloride $\left(\mathrm{CdCl}_{2}, 99.9 \%\right)$, selenium (pellets, 99.9\%), dodecylamine (DDA, 98\%), 1-hexanethiol (95\%), carbon disulfide $\left(\mathrm{CS}_{2}, 99.9 \%\right)$, aniline (99\%), trioctylphosphine oxide (TOPO, 95\%), trioctylphosphine (TOP, 90\%), hexadecylamine (HDA, 98\%), cadmium stearate, toluene (HPLC grade), acetonitrile (HPLC grade), chloroform (HPLC grade), hexane (HPLC grade), methanol (HPLC grade), sure seal dichloromethane (DCM) (>99\%), and concentrated ammonium hydroxide $\left(\mathrm{NH}_{4} \mathrm{OH}\right)(\mathrm{ACS}$ grade) were purchased from Sigma-Aldrich and used without further purification. Organic solvents were purged with $\mathrm{N}_{2}$ for 30 min prior to use.

Synthesis of Phenyldithiocarbamate Ligands. Phenyldithiocarbamate (PDTC) ligand was prepared according to a literature procedure [229]. Briefly, $82.0 \mathrm{mmol}$ of $\mathrm{CS}_{2}$ was added dropwise over $30 \mathrm{~min}$ to $41.0 \mathrm{mmol}$ of aniline dispersed in $30 \mathrm{~mL}$ of concentrated $\mathrm{NH}_{4} \mathrm{OH}$ at $0{ }^{\circ} \mathrm{C}$. The solution was stirred under $\mathrm{N}_{2}$ overnight. The resulting products for each aniline derivative appeared as suspended solids that ranged in color from yellow to white to gray. These products were washed with cold chloroform and dried under vacuum 
overnight. The PDTC ligand was stored in the dark before use. The products were characterized by ${ }^{1} \mathrm{H}$ NMR spectroscopy and electrospray ionization-mass spectrometry.

Synthesis of CdSe Nanoclusters. 1.6 diameter CdSe SCNCs [(CdSe $\left.)_{34}\right]$ were synthesized according to literature with slight modification [230]. Briefly, in a $100 \mathrm{~mL}$ twoneck round bottom flask, $0.140 \mathrm{~g}$ of $\mathrm{CdCl}_{2}$ mixed with $5 \mathrm{~mL}$ of DDA at $30^{\circ} \mathrm{C}$ until all solid dissolved. The optically clear solution was cooled to room temperature, and $10 \mathrm{~mL}$ of nitrogen-purged toluene was added before the addition of the selenium precursor. Selenium precursor was prepared separately by dissolving $0.06 \mathrm{~g}$ of freshly ground selenium powder in a mixture of $785 \mu \mathrm{L}$ of DDA and $215 \mu \mathrm{L}$ of 1-hexanethiol at $30{ }^{\circ} \mathrm{C}$ under $\mathrm{N}_{2}$. After addition of the selenium precursor, the reaction was stirred under $\mathrm{N}_{2}$ at $40{ }^{\circ} \mathrm{C}$ for $2 \mathrm{~h}$. After $2 \mathrm{~h}$, a stable absorption peak was observed, and the $\mathrm{SCNCs}$ were precipitated by the dropwise addition of nitrogen-purged acetonitrile. The yellow solid was collected by centrifugation. The precipitation step was repeated for two more times. Finally, the solid was dried with $\mathrm{N}_{2}$ and stored in the glovebox for further optical characterization and surface modification.

Synthesis of CdSe Quantum Dots. The CdSe QDs were synthesized according to literature procedure [57]. Briefly, 1.0 M trioctylphosphine selenide (TOPSe) solution was prepared inside the glovebox. $0.165 \mathrm{mmol}$ of cadmium stearate, $5.02 \mathrm{mmol} \mathrm{TOPO}$, and 8.03 mmol of HDA were loaded in a $100 \mathrm{~mL}$ three-neck round-bottom flask, and the mixture was heated at $320^{\circ} \mathrm{C}$ under nitrogen until an optically clear solution was appeared. At this point $1.0 \mathrm{~mL}$ of TOPSe was injected, and the reaction temperature was maintained at $290{ }^{\circ} \mathrm{C}$. When the QDs displayed an absorption maximum at $541 \mathrm{~nm}$, the reaction mixture was quenched by adding $10 \mathrm{~mL}$ of chloroform. CdSe QDs were purified by adding methanol. 
The purified product was stored inside the glovebox. According to an empirical formula [25], CdSe QDs displaying a $541 \mathrm{~nm}$ band-edge absorption peak have a diameter of $2.9 \mathrm{~nm}$. The size distribution of CdSe QDs used in the present work, as determined by a TEM image, is $2.9 \pm 0.2 \mathrm{~nm}$.

Ligand Exchange Reaction. The ligand exchange reaction was performed according to our published procedure [226]. Briefly, all samples were prepared inside a nitrogen-filled glovebox and reactions were carried out in a Schlenk line. $0.19 \mathrm{mmol}$ of DDA-coated CdSe SCNCs was dissolved in $10 \mathrm{~mL}$ of DCM in a $25 \mathrm{~mL}$ two-neck roundbottom flask followed by addition of $0.39 \mathrm{mmol}$ of PDTC ligand. The biphasic reaction mixture was vigorously stirred at room temperature for $48 \mathrm{~h}$. After the reaction mixture displayed its stable lowest energy absorption maximum, it was centrifuged, dried under reduced pressure, and stored inside a glovebox. The PDTC-passivated samples were stable at least a month inside the glovebox. The PDTC passivated CdSe SCNCs were completely soluble in DCM.

\subsubsection{Transient Absorption Spectroscopy.}

The transient absorption (TA) spectroscopy system [231] uses a regeneratively amplified mode-locked Ti:sapphire laser system (Clark-MXR, wavelength $=775 \mathrm{~nm}$, pulse duration $\lesssim 150 \mathrm{fs}$, pulse energy $\sim 1 \mathrm{~mJ}$ at $1 \mathrm{kHz}$ repetition rate). For the present TA experiment, pump sources with two different wavelengths were used: (i) second harmonics of the amplifier output $(388 \mathrm{~nm}$ ) by frequency doubling in a BBO crystal and (ii) output at $490 \mathrm{~nm}$ from a noncollinear optical parametric amplifier (NOPA), which was pulsecompressed to $\sim 30$ fs pulse duration. Output from the femtosecond laser amplifier $(775 \mathrm{~nm})$ 
was split and used to pump the doubling crystal, the NOPA, and a $3 \mathrm{~mm}$ thick sapphire plate to generate supercontinuum white light $(420-1600 \mathrm{~nm})$ which used as the probe source. Pulse energy of the pump light was attenuated to below $0.15 \mu \mathrm{J}$. The PDTC-passivated CdSe SCNCs in DCM were contained in a $1 \mathrm{~mm}$ thick quartz cuvette. The white-light probe beam was focused into the cuvette, while the pump beam was defocused to ensure the coverage of the probe beam in a noncollinear geometry. After the sample, the pump beam was blocked by a diaphragm, while transmission of the white-light probe beam was refocused onto the entrance slit of a spectrometer. It was then dispersed by a curved grating and detected by a linear array CMOS detector in the wavelength range of 430-730 nm. When $490 \mathrm{~nm}$ pump beam was used, an edge-pass filter was used to clean the residual pump beam radiation. The delay time between pump and probe pulses ( $\Delta \mathbf{t})$ was variable by moving a retroreflector on a computer-controlled translation stage which reflects the pump beam. An optical chopper revolving at $500 \mathrm{~Hz}$, half of the repetition rate of the femtosecond laser, was used to modulate the pump beam, and the TA signal was recorded as the change in optical density ( $\Delta$ OD) with pump beam blocked and unblocked:

$$
\Delta O D(\Delta t, \lambda)=-\log \left(\frac{I^{*}(\Delta t, \lambda)}{I_{0}}\right)
$$

Where $\boldsymbol{I}^{*}$ and $\boldsymbol{I}_{\mathbf{0}}$ are the probe beam transmission at each delay time with the preceding pump beam unblocked and blocked, respectively. Group velocity dispersion in the TA spectrum $\Delta \mathrm{OD}(\Delta \mathrm{t}, \lambda)$ was corrected using a power law function:

$$
\Delta t(\lambda)=\Delta t_{0}+A\left|\lambda-\lambda_{0}\right|^{p}
$$

In which $\lambda_{0}$ is the reference wavelength, and $\Delta t_{0}$ is the delay time at $\lambda_{0}$. Dozens of time zeros were pre-determined and plotted against their corresponding wavelengths in Origin. Then 
the parameters $\Delta \mathrm{t}_{0}, \mathrm{~A}, \lambda_{0}$, and $\mathrm{p}$ were determined by fitting the plot with a power law function. (See Appendix IV for details)

\subsection{Results and Discussions}

\subsubsection{Data Analysis and Exciton Dynamics}

Previously, our collaborators have shown that functionalization of ultrasmall CdSe SCNC surface with conjugated ligand PDTCs allowed excitonic hole delocalization [226]. It was observed that maximum delocalization took place for smaller ( $1.6 \mathrm{~nm}$ diameter) $\mathrm{CdSe}$ SCNCs because of matching between the energy levels of the SCNC and PDTC ligand orbitals. The delocalization of strongly confined holes to the ligand monolayer resulted in exitonic band gap reduction. The wavelength of the first excitonic transition of the PDTC passivated CdSe SCNCs was determined in UV-vis absorption measurement to be around $504 \mathrm{~nm}$ [226], corresponding to a bandgap of $\Delta \mathrm{Eg} \approx 2.46 \mathrm{eV}$. The steady states absorption spectrum is obtained by using Ocean Optics USB4000 Fiber Optic Spectrometer. The trace is shown at the top half of Figure 3.2, using Absorption (a.u.) as the y-axis.

Based on the previous experimental and theoretical results of Norris et al. [232], absorption at different wavelengths can be assigned to different excitonic transitions, which are better resolved in the transient absorption (TA) spectra (Figure 3.2). $388 \mathrm{~nm}$ laser beam was used as the pump source, and supercontinuum white light was used as probe source. The TA spectra were overlaid with steady states absorption spectrum, and shown at the bottom half of Figure 3.2. The change in optical density $(\Delta \mathrm{OD})$ on the $y$-axis indicates negative photobleaching $(\mathrm{PB})$ signal and positive photoabsorption (PA) signal. Different color schemes indicates TA spectrum at different delay time. At $\Delta t=0 \mathrm{ps}$, the PB signal 
starts rising and reach the maximum when $\Delta \mathrm{t}=1 \mathrm{ps}$, then slowly decays. The PB signal rising and decay times can be fit and calculated by plotting $\Delta \mathrm{OD}$ against $\Delta \mathrm{t}$ at varies wavelengths.

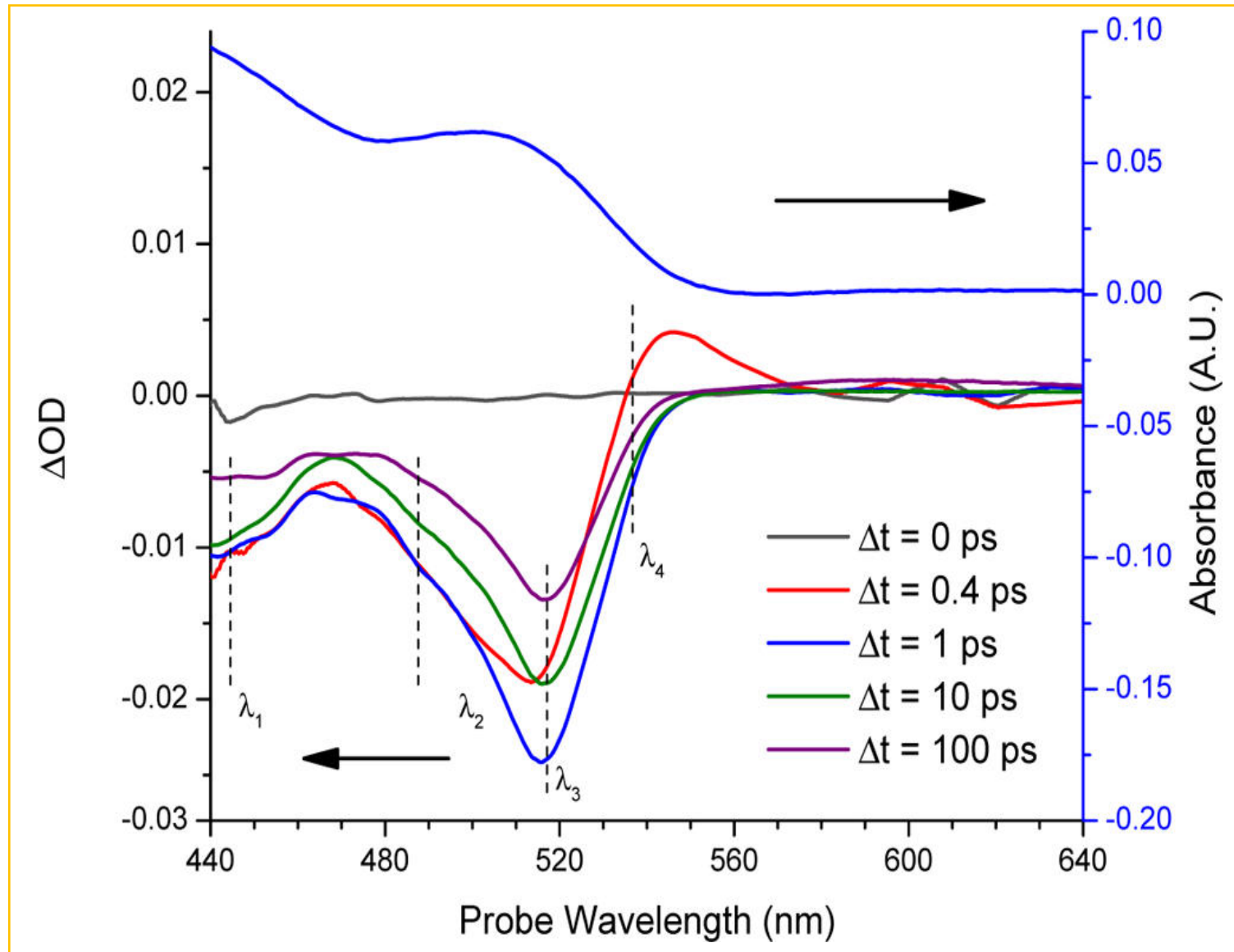

Figure 3.2. Steady-state absorption spectrum (top) and Transient absorption spectra at different pump-probe delay times (bottom) of PDTC-passivated CdSe SCNCs $(\mathrm{d}=1.6 \mathrm{~nm})$.

Pump beam wavelength: $388 \mathrm{~nm}$. Vertical dashed lines indicate the center wavelengths of TA spectra. Note that maximum of the positive photoabsorption (PA) signal is red-shifted from $\lambda_{4}$ to $\sim 545 \mathrm{~nm}$ due to strong influence by the negative photobleaching (PB) signal at $\lambda_{3}$.

The $388 \mathrm{~nm}$ pump source (photon energy $=3.20 \mathrm{eV}$, i.e., $0.74 \mathrm{eV}$ above bandgap) excites the $1 \mathrm{P}(\mathrm{e})-1 \mathrm{P}_{1 / 2}(\mathrm{~h})$ and $1 \mathrm{P}(\mathrm{e})-1 \mathrm{P}_{5 / 2}(\mathrm{~h})$ excitonic transitions, probably with contamination from the $1 \mathrm{~S}(\mathrm{e})-2 \mathrm{~S}_{1 / 2}(\mathrm{~h})$ transition. In the short wavelength region, the TA 
spectrum shows negative photobleaching $(\mathrm{PB})$ signal with three partially resolved peaks at $\lambda_{1}=445 \mathrm{~nm}(\mathbf{h} \boldsymbol{v}=2.79 \mathrm{eV}), \lambda_{2}=488 \mathrm{~nm}(\mathbf{h v}=2.54 \mathrm{eV})$, and $\lambda_{3}=517 \mathrm{~nm}(\mathbf{h v}=2.40 \mathrm{eV})$ that can be assigned to $1 \mathrm{~S}(\mathrm{e})-1 \mathrm{~S}_{1 / 2}(\mathrm{~h}), 1 \mathrm{~S}(\mathrm{e})-2 \mathrm{~S}_{3 / 2}(\mathrm{~h})$, and $1 \mathrm{~S}(\mathrm{e})-1 \mathrm{~S}_{3 / 2}(\mathrm{~h})$ transitions, respectively (Scheme 3.1).[201, 208, 233-235]

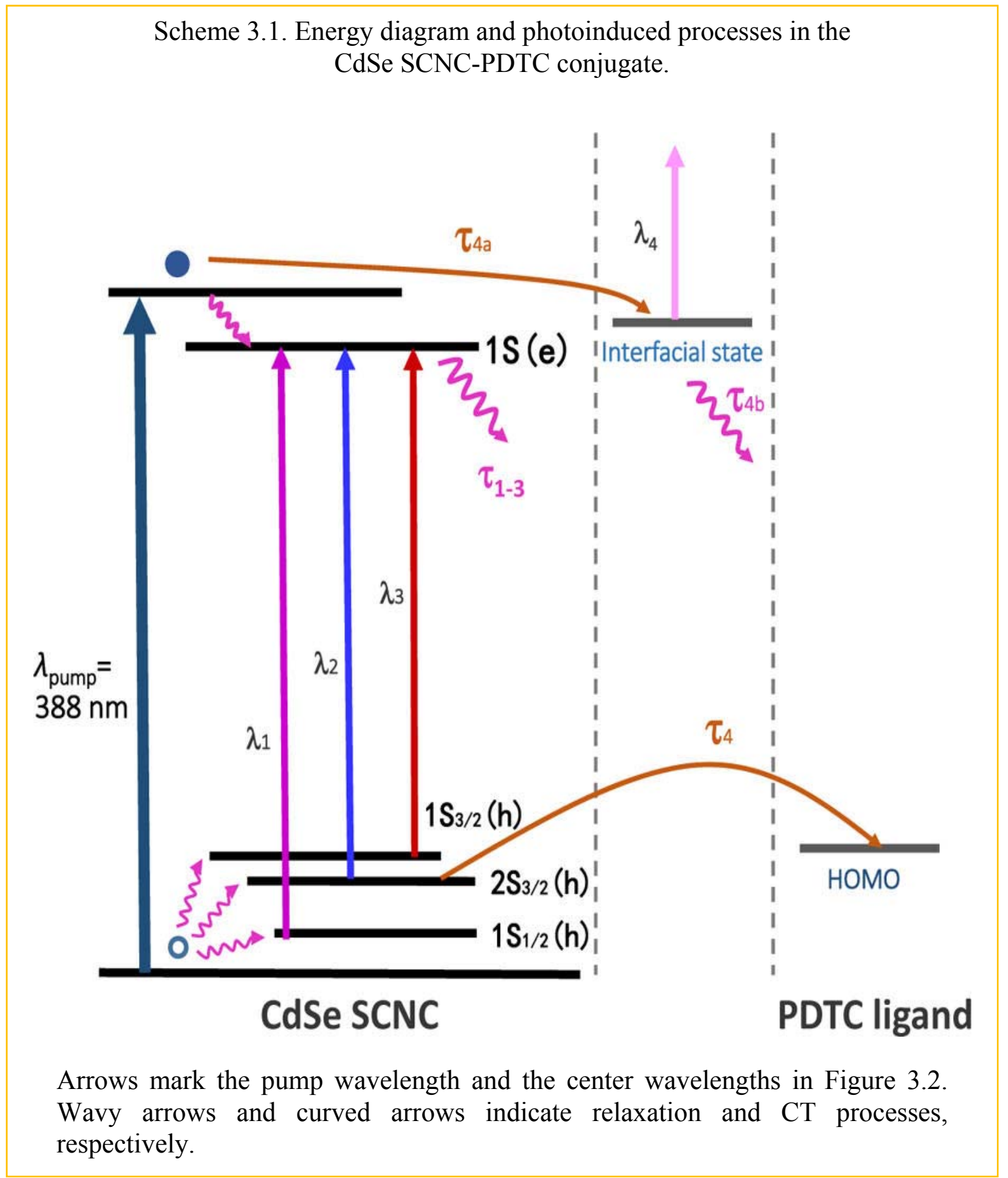


The PB signal at all three wavelengths is attributed to filling of the $1 \mathrm{~S}(\mathrm{e})$ electron state following photoexcitation of the $1 \mathrm{P}(\mathrm{e})-1 \mathrm{P}(\mathrm{h})$ transition that generates excitons and subsequent electron and hole relaxation. The exciton bleach recovery kinetics at these wavelengths are therefore similar to one another (Figure 3.3.a) and can be fit using a multiexponential decay function with time constants of $\tau_{1}=632 \mathrm{ps}, \tau_{2}=23 \mathrm{ps}$, and $\tau_{3}=2.7$ ps. (See Section 3.3.2 global fittings for details)

To better understand the underlying dynamics and interpret the observed ultrafast kinetics, control TA experiments with electron and hole quenchers $[44,236]$ were also performed. Benzoquinone (BQ) was added into the solution to quench electrons in photoexcited CdSe SCNCs. The aforementioned fast decay components $\left(\tau_{3}=2.7 \mathrm{ps}, \tau_{2}=23\right.$ ps) were significantly accelerated while the slow component ( $\left.\tau_{1}=632 \mathrm{ps}\right)$ was much less affected, as shown in Figure 3.4. In comparison, when pyridine (Py), a hole quencher, was added, the change of fast kinetics was insignificant at $455 \mathrm{~nm}$ and undetectable at 488 and $517 \mathrm{~nm}($ Figure 3.4.a-c). This confirms the previous observation that the exciton bleach signal is dominated by electrons rather than holes [27]. The slow and fast components are attributed to exciton recombination at the band edge $[237,238]$ and electron transfer to the passivating ligands, respectively.[208, 210]

More detailed comparison between the exciton bleach recovery kinetics at these three peak wavelengths reveals a unique fast decay component at $\lambda_{2}$ with a decay constant of $\tau_{4} \sim 900 \mathrm{fs}$ (Figure 3.3.b). The possibility of TA signal from the DCM solvent or PDTC ligands at $\lambda_{2}$ has been ruled out by negative results in control experiments using these samples. Sub-picosecond PB recovery process was previously observed in TA spectra of CdSe QDs $[239,240]$ and was attributed to electron relaxation following exciton generation. 


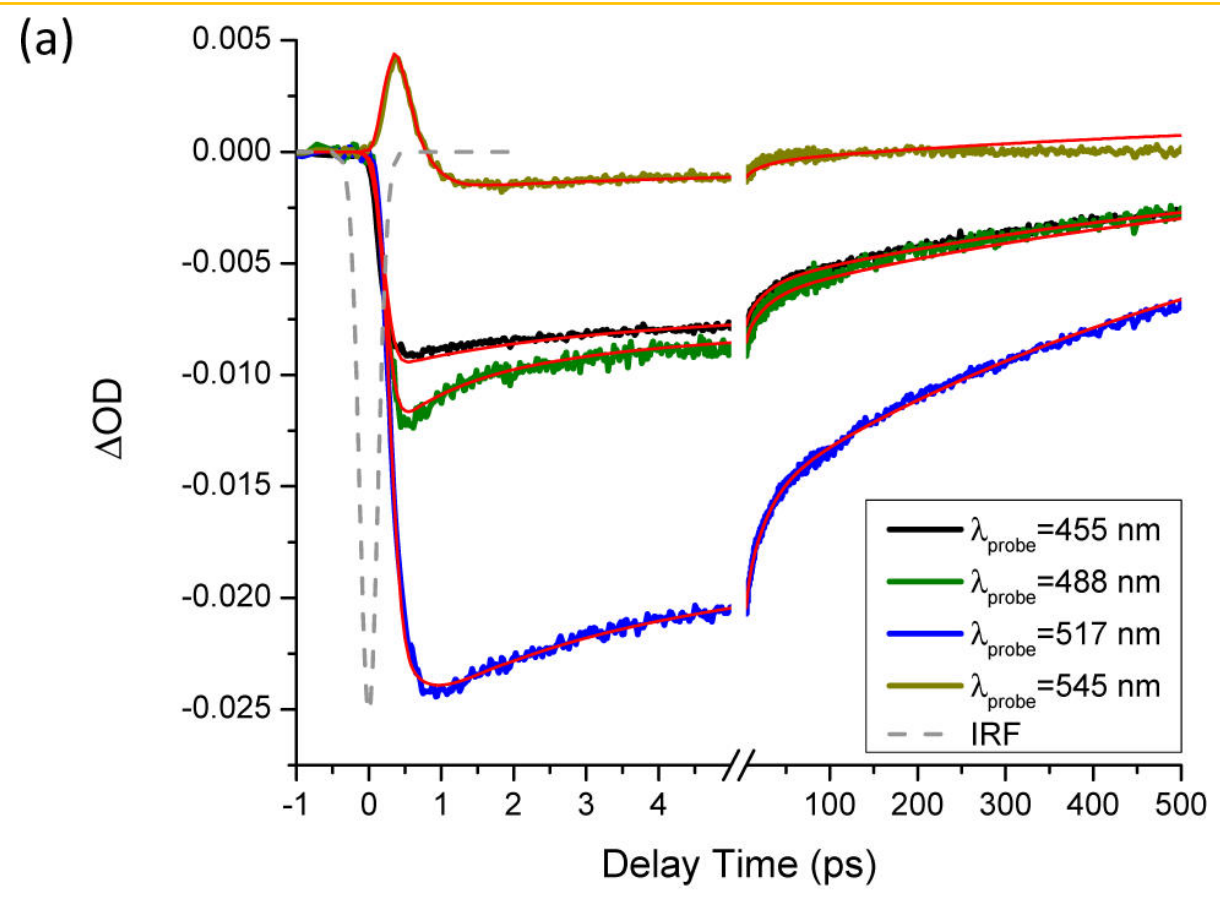

(b)

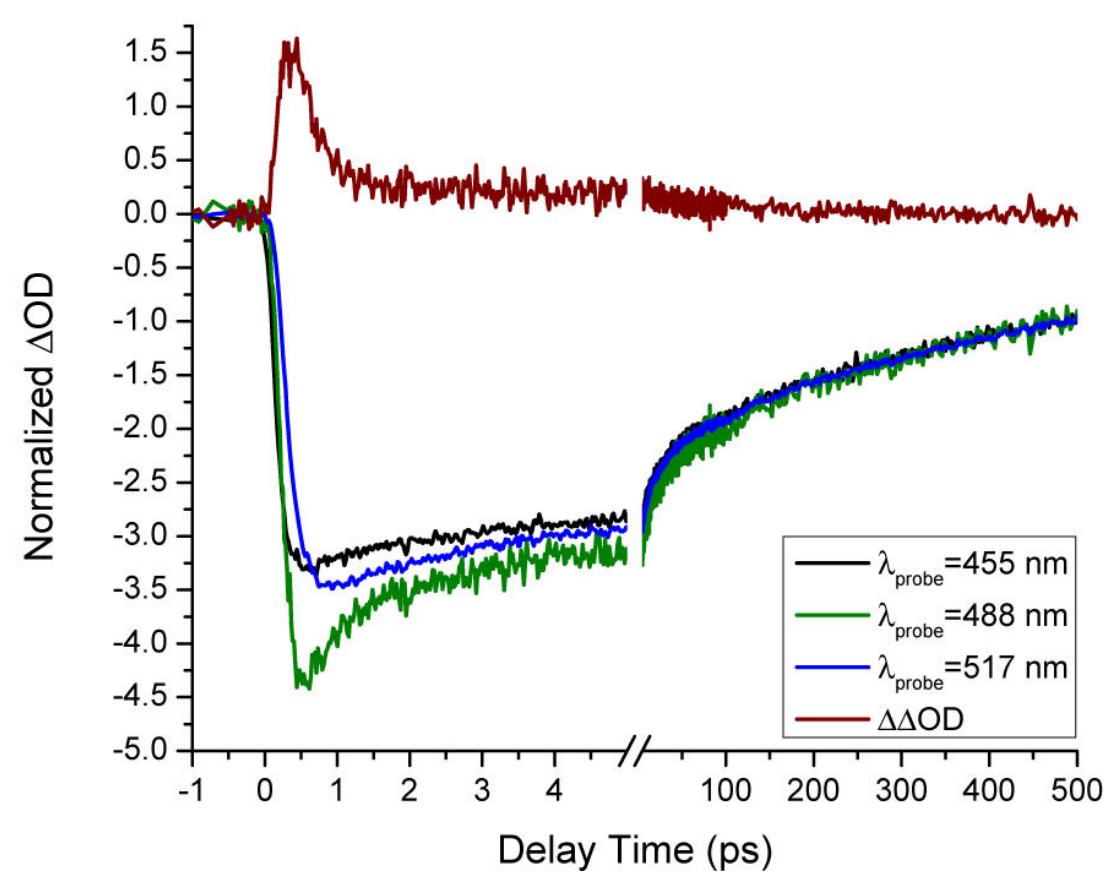

Figure 3.3 Time domain transient spectra of PDTC passivated $1.6 \mathrm{~nm} \mathrm{CdSe}$

(a) Ultrafast kinetics of the CdSe SCNC-PDTC conjugate at four peak wavelengths of the TA spectra. The dashed gray line represents instrument response function (IRF). The thin red lines are simulation using parameters determined in the global fitting (Table 3.1).

(b) Ultrafast kinetics TA spectra normalized to their slow decay component at long delay time. The positive trace $(\Delta \Delta \mathrm{OD})$ is subtraction of normalized kinetics at $488 \mathrm{~nm}$ from that at $517 \mathrm{~nm}$, which unravels the sub-picosecond hole transfer as described in the text. 
However, it was detected at the wavelength corresponding to the $1 \mathrm{P}(\mathrm{e})-1 \mathrm{P}_{3 / 2}(\mathrm{~h})$ transition, which is out of the detection range of the present experiment. At such wavelength, the growth of the PB signal in the delay time window of 0 to $400 \mathrm{fs}$ was assigned to the relaxation of electrons from higher energy levels to the $1 \mathrm{P}$ state, whereas the recovery of the PB signal ( $\tau=540 \mathrm{fs}$ ) was assigned to $1 \mathrm{P}$ to $1 \mathrm{~S}$ electron relaxation and is complementary to a secondary growth $(\tau=530 \mathrm{fs})$ at the wavelength of $1 \mathrm{~S}(\mathrm{e})-1 \mathrm{~S}_{3 / 2}(\mathrm{~h})$ transition. In the present work of CdSe SCNC- PDTC ligand conjugates, the sub-picosecond PB recovery process was observed at $\lambda 2$ exclusively, corresponding to the $1 \mathrm{~S}(\mathrm{e})-2 \mathrm{~S}_{3 / 2}(\mathrm{~h})$ transition. Electron relaxation is therefore unlikely to be the underlying mechanism.

Because transients at $\lambda_{1}, \lambda_{2}$, and $\lambda_{3}$ share the same electron state, the observed difference in the bleach recovery kinetics can only be explained by different dynamics involving the hole states. $\lambda_{2}$ is the center wavelength of the $1 \mathrm{~S}(\mathrm{e})-2 \mathrm{~S}_{3 / 2}(\mathrm{~h})$ transition. The sub-picosecond decay component is therefore attributed to hole transfer from the $2 \mathrm{~S}_{3 / 2}(\mathrm{~h})$ level of the CdSe SCNC to the HOMO level of the PDTC ligand. According to previous effective mass approximation calculation [40] and electrochemical measurements [19, 241, 242], the valence band edge of $1.6 \mathrm{~nm}$ CdSe SCNCs would be $\mathrm{E}_{\mathrm{b}}=-6.16 \mathrm{eV}$. The HOMO energy of PDTC is calculated to be $\sim-6.15 \mathrm{eV}$ [56]. This energetic alignment between the valence band of CdSe SCNC and the HOMO of PDTC ligand enhances the possibility of strong interfacial orbital mixing and formation of hybrid orbitals, as illustrated in Scheme 3.1, a curved arrow labeled $\tau_{4}$ indicates the charge transfer process from $2 \mathrm{~S}_{3 / 2}(\mathrm{~h})$ level of CdSe to the HOMO of PDTC ligand. Thus, strongly confined hole wave functions of ultrasmall CdSe SCNCs can expand beyond the inorganic core boundary to the ligand monolayer through hybrid orbitals. Coupling and hybridization between the lowest-energy 
hole states of CdSe SCNC and the ground state of PDTC ligand is therefore expected [56, 57, 243], which facilitates exciton relaxation and hole transfer [51].
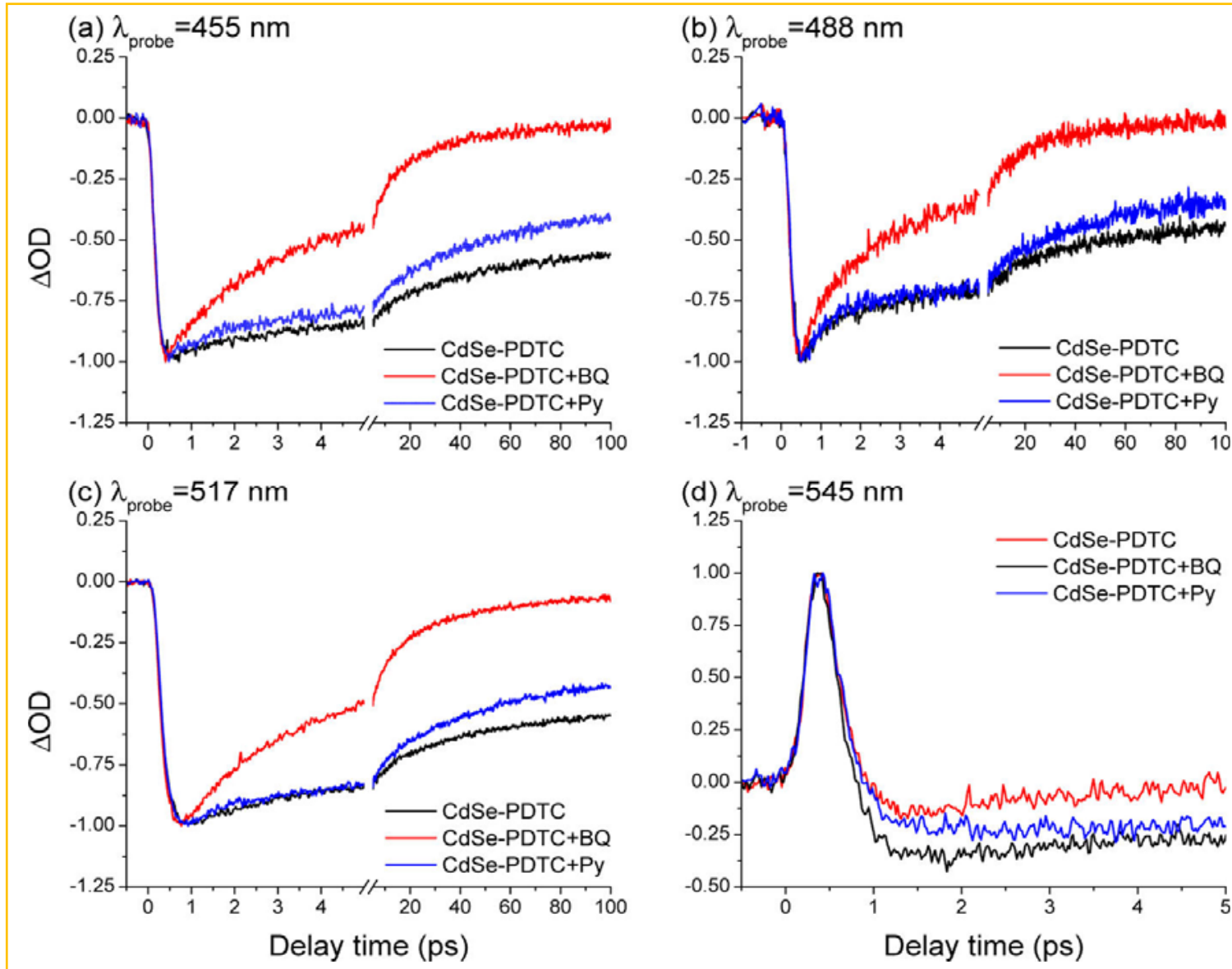

Figure 3.4. Ultrafast kinetics of PDTC-passivated CdSe SCNCs at four wavelengths with electron quencher (Red trace) or hole quencher (Blue trace).

Benzoquinone (BQ) is used as electron quencher and pyridine (Py) is used as hole quencher. The three time decay kinetics at each wavelength are normalized to their maximum $\triangle \mathrm{OD}$.

Although PB signal due to state filling that involves three hole states $\left(1 \mathrm{~S}_{3 / 2}, 2 \mathrm{~S}_{3 / 2}\right.$, $1 \mathrm{~S}_{1 / 2}$ ) were detected, only hole transfer from the $2 \mathrm{~S}_{3 / 2}(\mathrm{~h})$ state was observed. This is probably due to the larger extension of the $2 \mathrm{~S}_{3 / 2}(\mathrm{~h})$ orbital of the CdSe SCNC and hence better overlap with the HOMO of the PDTC ligand. It is worth noting that for CdSe QDs, the PB signal in 
the TA spectra is mainly due to the photo-excited electrons residing in the lowest energy 1S(e) state, whereas the contribution of holes is usually insignificant [27]. The observation of hole transfer in the present experiment on SCNCs is attributed to the relatively high excitation energy and the relatively large energy separation between hole states due to the ultrasmall size of the SCNCs.

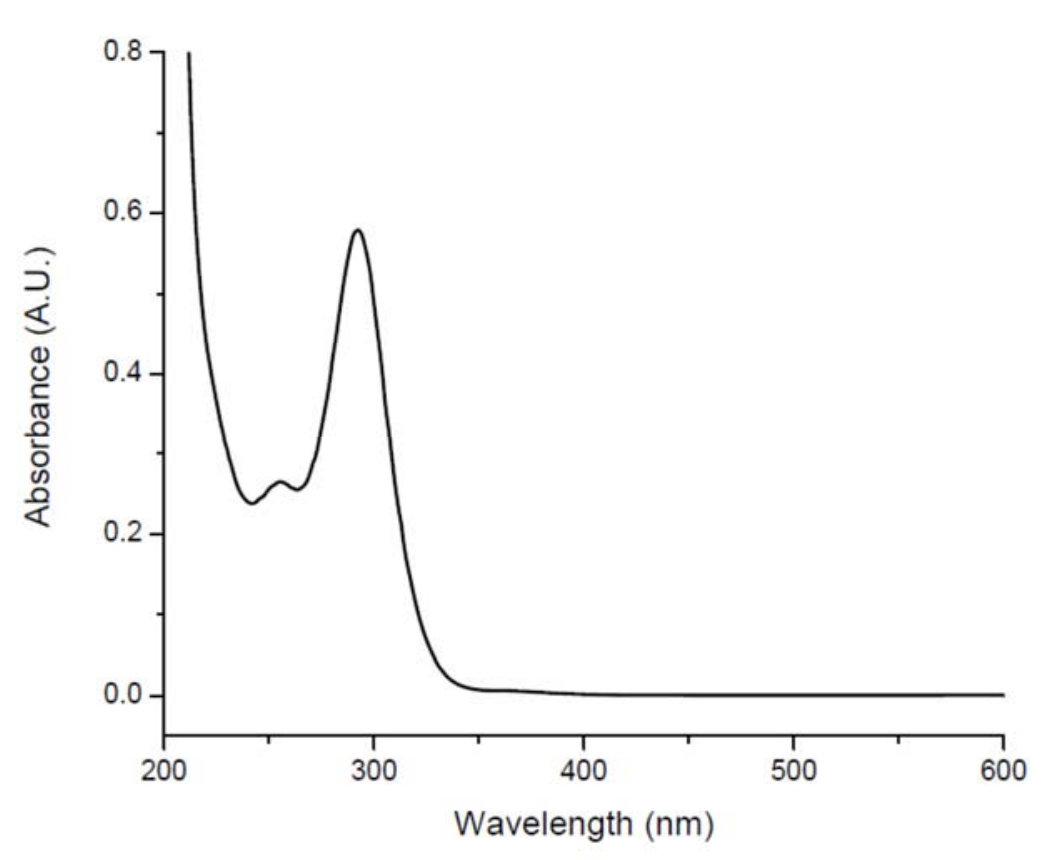

Figure 3.5. Steady-states absorption spectrum of PDTC in water.

In the longer wavelength region, the TA spectrum of CdSe SCNC-PDTC conjugates possesses a positive photoabsorption (PA) signal centered at $\lambda_{4}=536 \mathrm{~nm}(\mathbf{h v}=2.31 \mathrm{eV}$, see Figure 3.2). In our control experiments, the PDTC molecule does not have absorption at $388 \mathrm{~nm}$ (Figure 3.5), and no TA signal of PDTC in DCM was observed. Previously, positive signals in QD systems in the long wavelength region were assigned to exciton-exciton interactions (in CdSe QDs, $\mathrm{r} \sim 5.7 \mathrm{~nm}$ ) [27, 239] or to absorption of charged free radicals resulted from electron transfer (in CdSe/ CdS QD- $\mathrm{MV}^{2+}$ conjugates) [44]. 

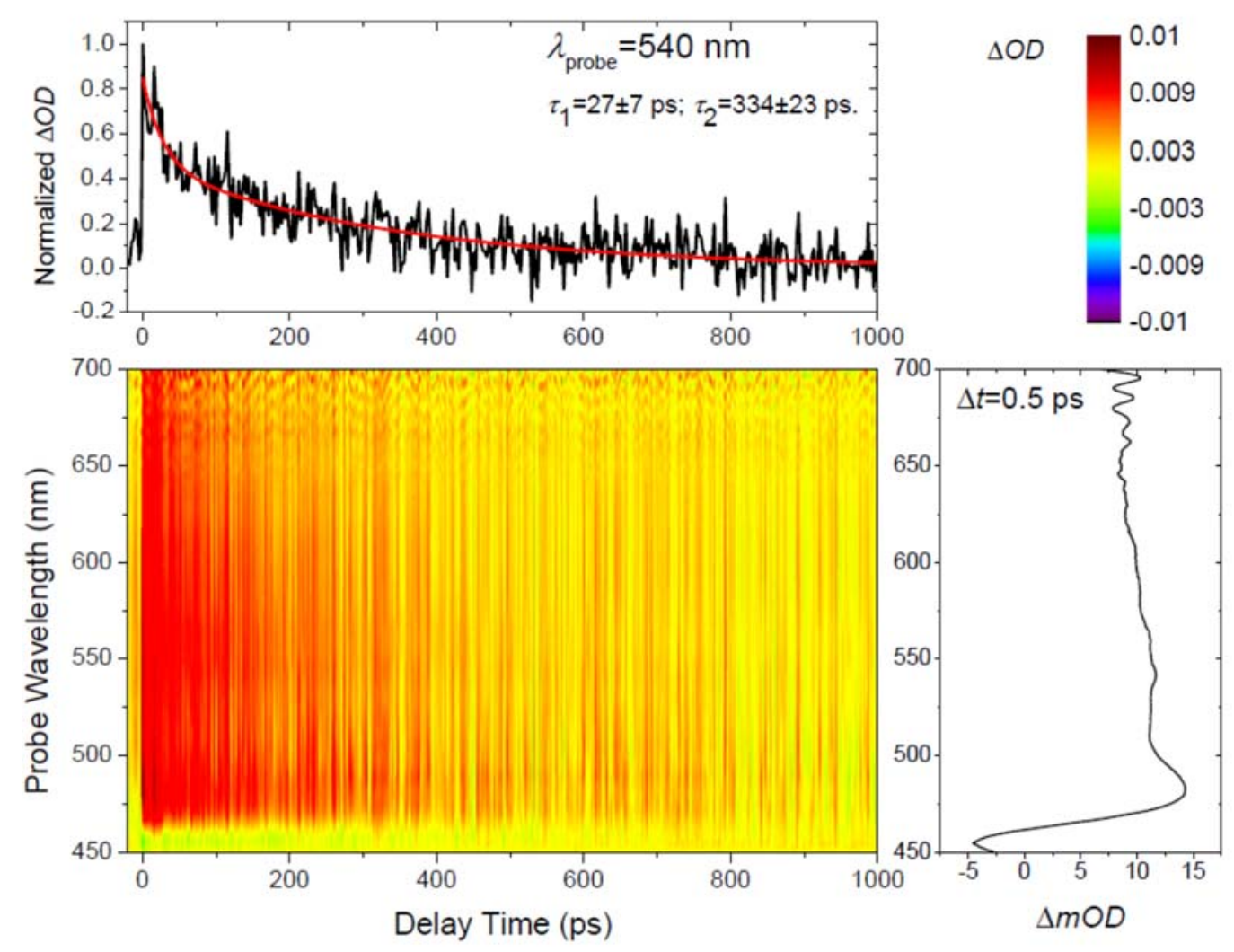

Figure 3.6. Three dimensional TA spectrum of DDA-coated CdSe SCNCs with DCM as solvent and pumped at $388 \mathrm{~nm}$.

Upper left panel: decay dynamics at $540 \mathrm{~nm}$ probe wavelength. Red line shows a fit to biexponential decay. Lower right panel: Wavelength dimension TA spectrum at $0.5 \mathrm{ps}$ decay time.

In order to unravel the underlying mechanism of the PA signal, TA spectroscopy was applied to investigate the photoinduced dynamics of $1.6 \mathrm{~nm}$ diameter $\mathrm{CdSe} \mathrm{SCNCs}$ passivated by PDTC ligands, which, unlike PDTC ligands, are not strongly coupled to the CdSe cores because of mismatch of the energy levels. The TA spectrum of DDA-passivated CdSe SCNCs (shown in Figure 3.6) is dominated by broad PA signal in the longer wavelength region (>460 nm). The PB signal due to state filling is almost completely out of the detection range of the array detector although weak negative signal at the shorter 
wavelength edge of the detection range $(\sim 450 \mathrm{~nm})$ was observed. The ultrafast dynamics of the PA signal can be fit to a biexponential decay with time constants much longer than $1 \mathrm{ps}$. A similar broad and long-lived PA signal was previously observed for ligand protected QDs [208, 210] and was attributed to red-shifted 1S-1S excitonic transitions due to exciton-exciton interaction. This PA signal of DDA-coated CdSe SCNCs, however, is significantly different from the PA signal that was observed for CdSe SCNC-PDTC ligand conjugates, which is much narrower in the wavelength domain and is of sub-picosecond time scale (Figure 3.2 and Figure 3.3.b). Exciton-exciton interaction is, therefore, unlikely to be the underlying mechanism of the PA signal centered at $\lambda_{4}$.

The positive TA signal cannot be attributed to radical cations either: Its subpicosecond time scale is too short compared to that of the radical cation signal (on the order of $100 \mathrm{~ns}$ as reported previously[44]). Moreover, unlike the PA signal of radical cations [44], the positive signal of the CdSe SCNC-PDTC conjugate at $\sim 545 \mathrm{~nm}$ did not disappear when the electron quencher BQ was added (Figure 3.4.d). It is therefore attributed to new charge transfer states (CTSs) created by charge separation across the SCNC-ligand interface. CTSs are usually formed within 0.1 ps.[34, 244, 245] which is consistent with the observation of the present experiment. They are then populated via hot electron transfer upon photoexcitation of $388 \mathrm{~nm}$. No positive signal was observed when lower pump photon energy was used (for instance, $\lambda=490 \mathrm{~nm}$ ). As shown in Figure 3.7, when $490 \mathrm{~nm}$ pump power is used, the positive PA signal in the long wavelength region is no longer present. The decay dynamics at $545 \mathrm{~nm}$ probe wavelength further supports the absence of PA signal when lower pump photon energy was used. 


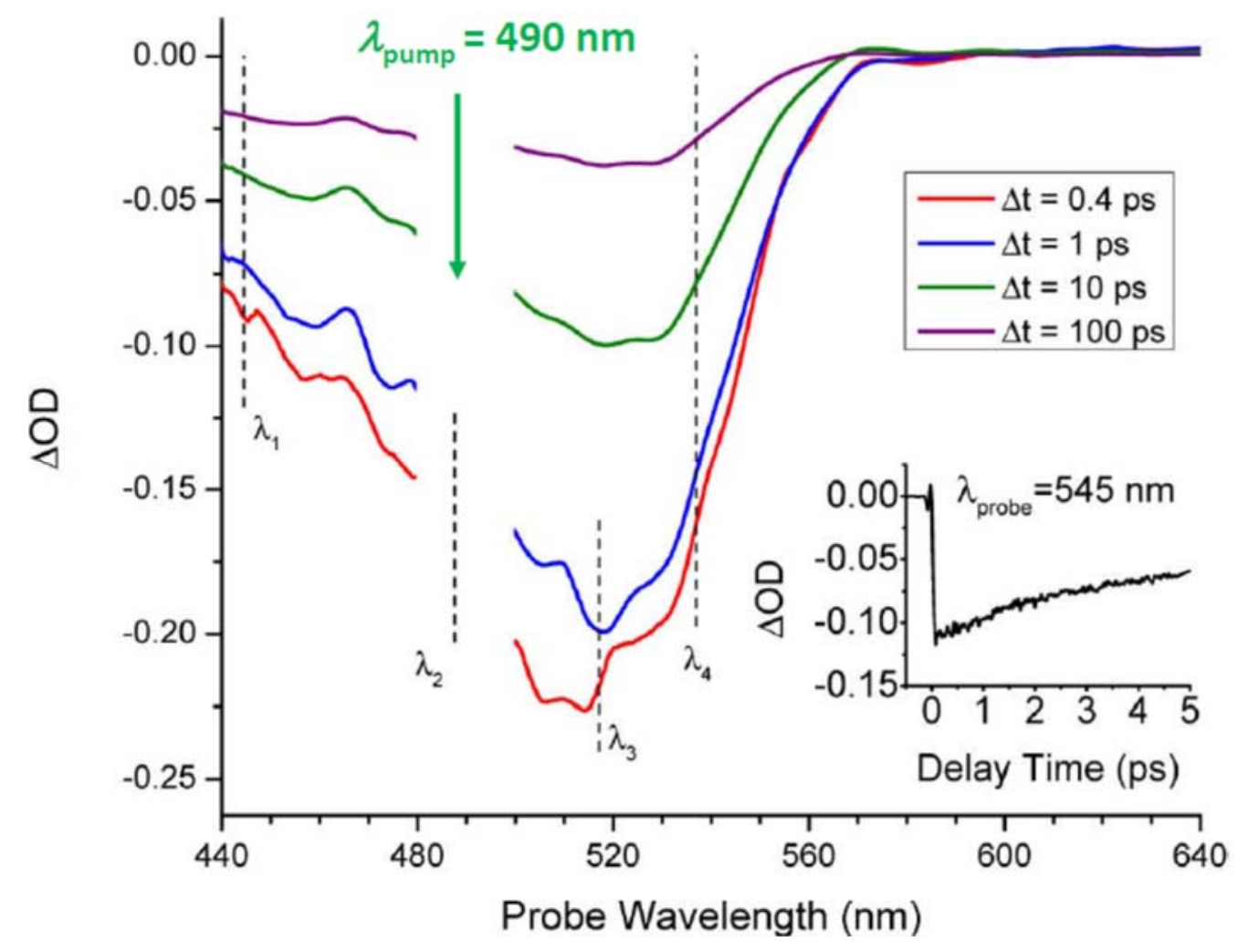

Figure 3.7. TA spectra of PDTC-passivated $1.6 \mathrm{~nm}$ CdSe SCNCs with $490 \mathrm{~nm}$ pump wavelength. (Inset shows decay kinetics at $545 \mathrm{~nm}$ ).

Such a comparison suggests that the interfacial CTSs involved in the observed hot electron transfer process are above the conduction band edge of CdSe SCNCs, which facilitates strong electronic coupling and allows hot electron transfer to PDTC ligands. Energy level alignment by itself, however, does not ensure hot electron transfer. Following excitation by high energy photons, hot charge carrier transfer is in competition with and may be outrun by relaxation processes. Observation of sub-picosecond hot electron transfer in the SCNC-PDTC conjugates implies that the hot electron transfer rate is comparable to that of the relaxation processes. This is attributed to large energy spacing between electron states of the inorganic core and the well-engineered nanocluster surface-ligand interface that removes electron trap states, both of which slow down electron cooling. 
Time evolution of the PA signal can be well-simulated using a two-step consecutive reaction model, in which the intensity of PA matches the population of the interfacial states (see Section 3.2.2 for details). The growth of the PA signal (with rate constant $\mathrm{k}_{4 \mathrm{a}}=5.37$ $\left.\mathrm{ps}^{-1}\right)$ corresponds to hot electron transfer to the interfacial state, while its decay $\left(\mathrm{k}_{4 \mathrm{~b}}=5.45\right.$ $\mathrm{ps}^{-1}$ ) may be attributed to ultrafast recombination and relaxation processes. Electronic coupling between the orbitals of the CdSe SCNCs and the passivating ligands depends on their energy level structures. Modification of either SCNCs or ligands can therefore affect the charge transfer processes.

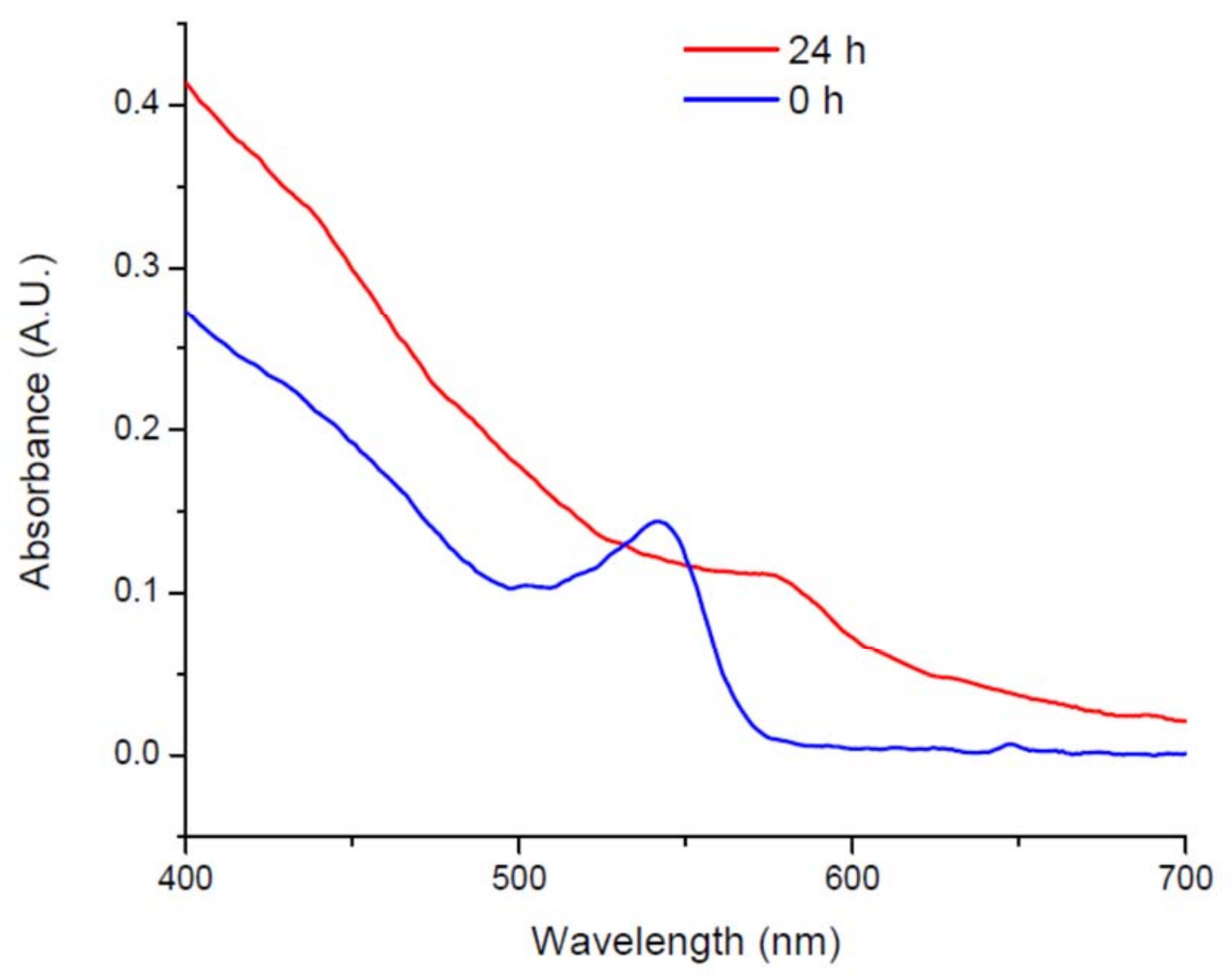

Figure 3.8. Steady States Absorption Spectra of $2.9 \mathrm{~nm}$ diameter CdSe QDs

Blue trace $(\lambda=541 \mathrm{~nm})$ is the spectrum of $2.9 \mathrm{~nm}$ "naked" CdSe QDs, and the red trace $(\lambda=578 \mathrm{~nm})$ is PDTC-CdSe QDs. The red shift and broadening of the absorption spectrum after ligand exchange indicates exciton delocalization from the CdSe QDs to ligand monolayers. 
(a)

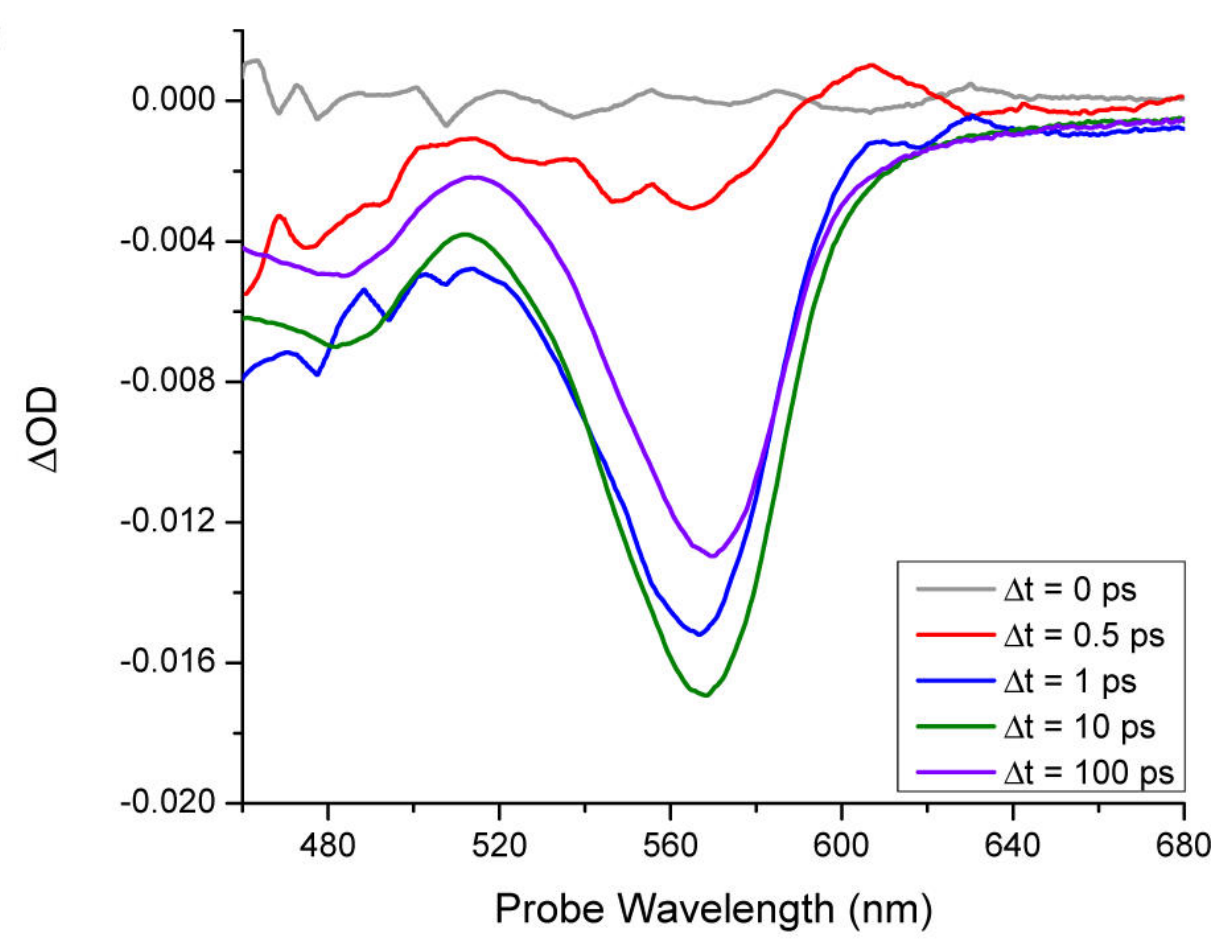

(b)

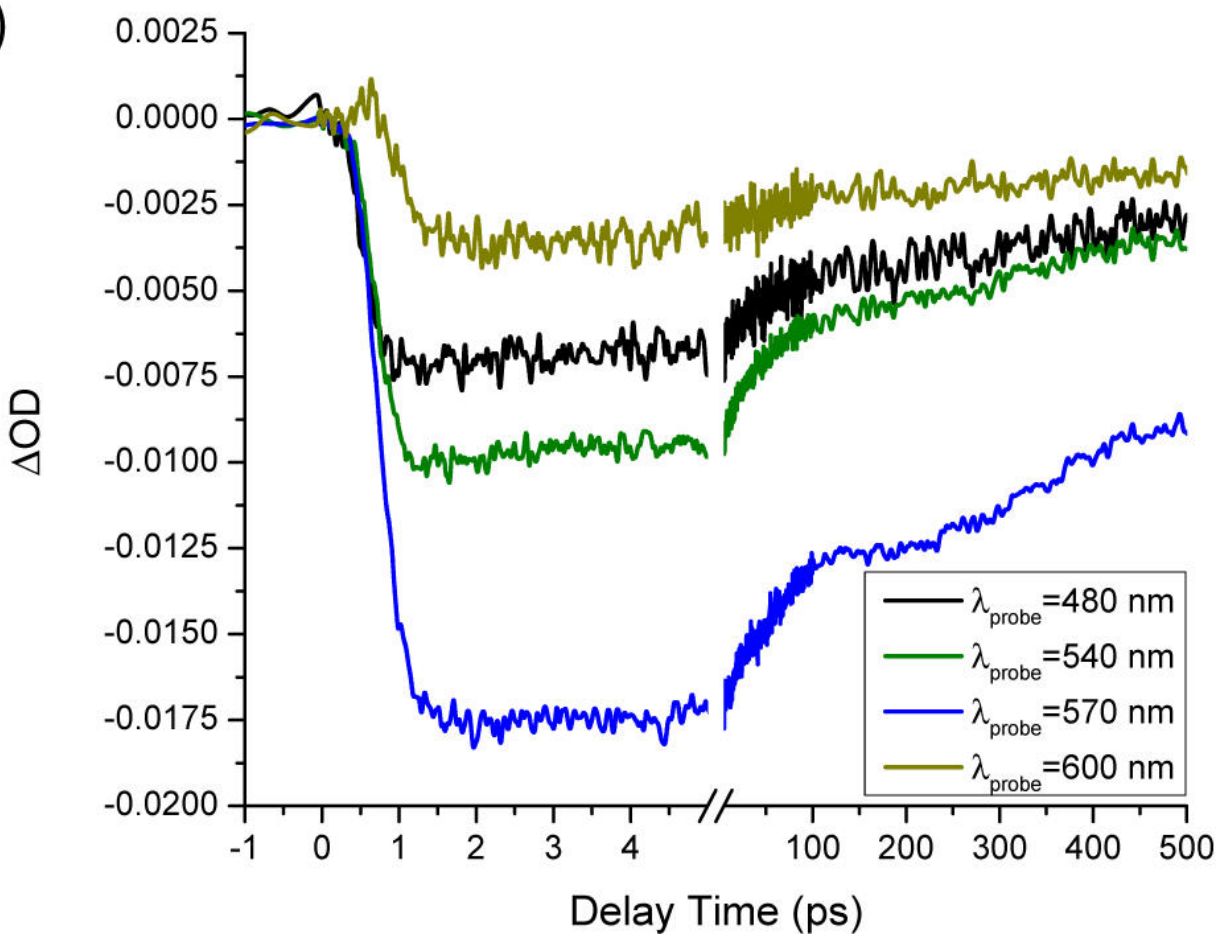

Figure 3.9. Transient Absorption (TA) Spectra of CdSe QDs-PDTC ligand conjugate

The diameter of the QDs is $2.9 \mathrm{~nm}$. The bandgap energy is $2.15 \mathrm{eV}(\lambda=578 \mathrm{~nm})$. Wavelength domain of the TA spectra at different pump-probe delay times is shown in (a). Ultrafast time domain of the TA spectra illustrate the decay kinetics at four wavelengths is shown in (b). 
Previously, Teunis and coworkers have shown that exciton delocalization in CdSe SCNC decreased as its size was increased [226]. Based on this observation, different TA signals are expected for QDs. To further explore the ultrafast hole and electron transfer processes, we varied the sizes of nanocrystals, while PDTC surface coating remained unchanged. When PDTC passivated CdSe QDs with $\mathrm{d}=2.9 \mathrm{~nm}$ and $\mathrm{Eg}_{\mathrm{g}}=2.15 \mathrm{eV}(\lambda=578$ nm, see Figure 3.8 for steady-state absorption spectrum) were used to investigate the excited state dynamics, different TA spectra were observed, as shown in Figure 3.9. The positive signal in the long-wavelength region is significantly weaker compared to that of the $1.6 \mathrm{~nm}$ CdSe SCNCs (Figure 3.2). The sub-picosecond decay component at $\lambda_{2}$ (with time constant $\left.\tau_{4}\right)$ is almost absent. Such observation supports the attribution of the PA signal at $\lambda_{4}$ to charge transfer rather than exciton-exciton interaction because magnitude of the PA and PB signal due to exciton-exciton interaction is insensitive to the size of QDs or SCNCs [240]. Moreover, it suggests that both hot electron and hole transfer processes are sensitive to energy level alignment.

\subsubsection{Global Fitting and Simulations}

To unravel the exciton dynamics and derive quantitative information about charge transfer processes, experimentally obtained three dimensional transient absorption (TA) spectra were fit to a model that takes into account the ultrafast processes at all four center wavelengths $\lambda_{1} \sim \lambda_{4}$. A function of $\Delta \mathrm{OD}$ which has two variables $(\lambda, \Delta \mathrm{t})$ attributes to both wavelength domain and time domain. 


$$
\Delta O D(\lambda, \Delta t)=\left\{\begin{array}{l}
\sum_{i=1}^{3}\left[A_{i}(\lambda) \frac{\sum_{j=1}^{3 o r} \gamma_{j} e^{-\Delta t / \tau_{j}}}{\sum_{j=1}^{3 o r} \gamma_{j}}\right] \\
A_{4}(\lambda)\left[\frac{k_{4 a}\left(e^{-k_{4 a} \Delta t}-e^{-k_{4 b} \Delta t}\right)}{k_{4 b}-k_{4 a}}\right]
\end{array}\right\} \otimes \operatorname{IRF}(\Delta t)
$$

In which $A_{i}(\lambda)$ indicates the wavelength domain of the spectral profile, which was simulated by a multi-peak Gaussian line shape. Each component can be written as the equation below. Where $\lambda_{\mathrm{i}}(\mathrm{i}=1 \sim 4)$ are the center wavelengths of transient absorption.

$$
A_{i}(\lambda)=\frac{Y_{i}}{\sigma \sqrt{2 \pi}} e^{-\left(\frac{1}{2}\right)\left[\frac{\left(\lambda-\lambda_{i}\right)}{\sigma}\right]^{2}}
$$

The ultrafast time decay kinetics at $\lambda_{1} \sim \lambda 3$ are fit to a multi-exponential decay with three (at $\lambda_{1}$ and $\lambda_{3}$ ) or four (at $\left.\lambda_{2}\right)$ components with different weights $\left(\gamma_{j}\right)$ and time constants $\left(\tau_{\mathrm{j}}\right)$. At wavelength $\lambda_{1}$ and $\lambda_{3}$, which corresponding to $1 \mathrm{~S}(\mathrm{e})-1 \mathrm{~S}_{1 / 2}(\mathrm{~h})$ and $1 \mathrm{~S}(\mathrm{e})-1 \mathrm{~S}_{3 / 2}(\mathrm{~h})$ transitions, respectively, there are one slow decay component, $\tau_{1}$, and two fast decay components, $\tau_{2}$ and $\tau_{3}$. While at wavelength $\lambda_{2}$, an unique sub-picosecond time component $\tau_{4}$ was put into the equation to simulate the fast decay observed at $\lambda_{2}$ corresponding to $1 \mathrm{~S}(\mathrm{e})-2 \mathrm{~S}_{3 / 2}(\mathrm{~h})$ transitions (see Figure 3.3.b).

At wavelength $\lambda_{4}$ the positive PA signal is fit to a two-step consecutive reaction model, and then multiply the wavelength domain function $\mathrm{A}_{4}$, (see the second term in the curly brackets of $\Delta \mathrm{OD}$ function). In simulating the 3D TA spectra, ultrafast kinetics are convolved with Instrument Response Function (IRF). With $\sigma_{\text {IRF }}=127$ fs. To save computation time, convolution was performed only in the region of $\Delta \mathrm{t}=-0.5$ to $5 \mathrm{ps}$. A 
small positive offset had to be added to $A_{4}(\lambda)$, in order to compensate the strong influence by the negative signal at $\lambda_{3}$.

$$
\operatorname{IRF}(t)=\frac{Y_{I R F}}{\sigma_{I R F} \sqrt{2 \pi}} e^{-\left(\frac{1}{2}\right)\left(\frac{t}{\sigma_{I R F}}\right)^{2}}
$$

All the simulation equations above were put into Mathcad software, and a global fitting was performed to calculate all the time kinetics variables, as listed in Table 3.1. Details about global fitting and Mathcad code can be found in Appendix II.

Table 3.1. Parameters Determined in the Global Fitting of the TA Spectra.

\begin{tabular}{|c|c|c|c|}
\hline$\lambda_{1}$ & $\lambda_{2}$ & $\lambda_{3}$ & $\lambda_{4}$ \\
\hline $445 \mathrm{~nm}$ & $488 \mathrm{~nm}$ & $517 \mathrm{~nm}$ & $536 \mathrm{~nm}$ \\
\hline$Y_{1}$ & $Y_{2}$ & $\gamma_{3}$ & $\boldsymbol{Y}_{\mathbf{4}}$ \\
\hline-0.43 & -0.38 & -0.92 & 1.37 \\
\hline$\tau_{1}$ & $\tau_{\mathbf{2}}$ & $\tau_{\mathbf{3}}$ & $\tau_{\mathbf{4}}$ \\
\hline $632 \mathrm{ps}$ & $23 \mathrm{ps}$ & $2.7 \mathrm{ps}$ & $0.9 \mathrm{ps}$ \\
\hline$\gamma_{1}$ & $\gamma_{\mathbf{2}}$ & $\gamma_{\mathbf{3}}$ & $\gamma_{\mathbf{4}}$ \\
\hline 1 (fixed) & 0.32 & 0.32 & 0.62 \\
\hline $\boldsymbol{k}_{4 \mathrm{a}}$ & $\boldsymbol{k}_{4 \mathrm{a}}$ & & \\
\hline $5.37 \mathrm{ps}^{-1}$ & $5.45 \mathrm{ps}^{-1}$ & & \\
\hline$\sigma$ & $\sigma_{\text {IRF }}$ & & \\
\hline $14 \mathrm{~nm}^{127 \mathrm{fs}}$ & & & \\
\hline
\end{tabular}


$1 \mathrm{~S}(\mathrm{e})-1 \mathrm{~S}_{1 / 2}(\mathrm{~h}), 1 \mathrm{~S}(\mathrm{e})-2 \mathrm{~S}_{3 / 2}(\mathrm{~h})$, and $1 \mathrm{~S}(\mathrm{e})-1 \mathrm{~S}_{3 / 2}(\mathrm{~h})$ transitions share three time decay components, $\tau_{1}=632 \mathrm{ps}, \tau_{2}=23 \mathrm{ps}$, and $\tau_{3}=2.7 \mathrm{ps}$. What unique about $1 \mathrm{~S}(\mathrm{e})-2 \mathrm{~S} 3 / 2(\mathrm{~h})$ transition, is that contains an ultrafast time components $\tau_{4}=0.9 \mathrm{ps}$, attributes to the fast hole transfer from CdSe SCNCs toward the PDTC ligand orbitals, which we discussed in the previous section.

Experimentally obtained TA spectra of $1.6 \mathrm{~nm}$ CdSe SCNC-PDTC conjugates were simulated using the time constants, $\tau_{1} \sim \tau_{4}$, and $\mathrm{k}_{4 \mathrm{a}}=5.37 \mathrm{ps}^{-1}, \mathrm{k}_{4 \mathrm{~b}}=5.45 \mathrm{ps}^{-1}$, alone with other parameters, which were determined in the global fit. The fit ultrafast time kinetics (time domain of the TA spectra) are previously shown in Figure 3.3, while the fit TA spectra in the wavelength domain are compared to experimental ones in Figure 3.10.

\subsection{Conclusions}

In conclusion, we have studied the excited-state charge transfer dynamics in molecule-like CdSe SCNCs coated with conjugated PDTC ligands. Sub-picosecond PA signal was detected at a wavelength that is longer than the $1 \mathrm{~S}-1 \mathrm{~S}$ excitonic transition. $\mathrm{PB}$ recovery dynamics were observed at the wavelengths of three excitonic transitions that involve the $1 \mathrm{~S}$ electron state. In addition, an extra sub-picosecond PB recovery process is distinguishable at the $1 \mathrm{~S}(\mathrm{e})-2 \mathrm{~S}_{3 / 2}(\mathrm{~h})$ transition wavelength. Control experiments have been carried out in order to unravel the underlying mechanism for the sub-picosecond PA and PB processes. Based on the experimental results, it is unlikely that the sub-picosecond dynamics are due to exciton-exciton interaction and the electron relaxation process. The subpicosecond PA and PB recovery processes are attributed to hot electron transfer (ET) and hole transfer (HT) from the CdSe SCNC core to the ligand monolayer, respectively. The HT 
process is subject to strong interfacial orbital mixing and formation of hybrid orbitals between the valence band orbitals of SCNC and the HOMO of the PDTC ligands,[56, 57, 227] while the sub-picosecond ET process is attributed to hot electrons transferred to interfacial charge transfer states (CTSs) following photoexcitation using $388 \mathrm{~nm}$ pump source.

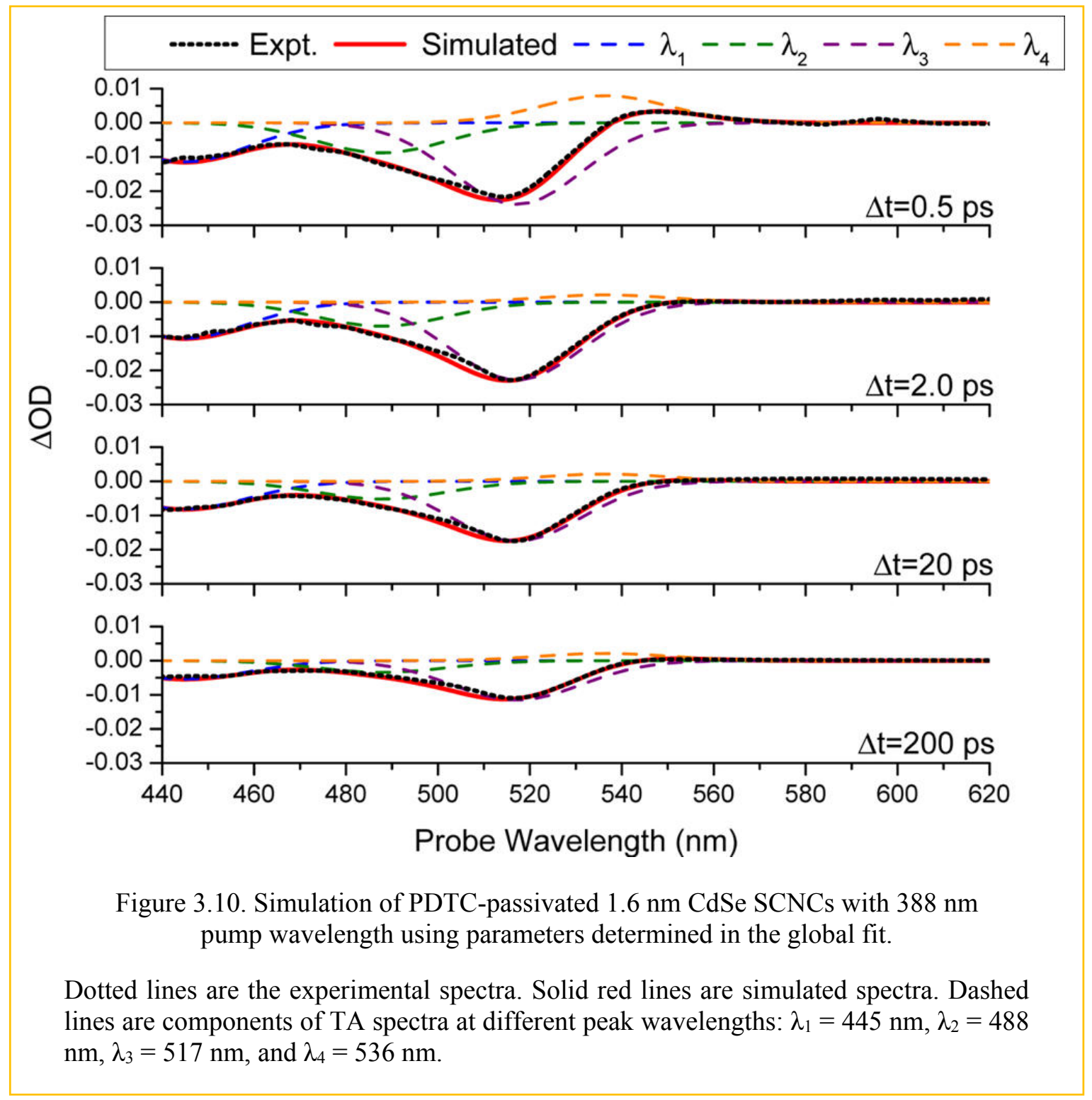


The present investigation will provide important guidelines in the preparation of highly efficient electronic materials for the design of optoelectronic devices as follows:

(1) Various types of QDs are commonly used as sensitizers in photovoltaic cells where fast transfer and stabilization of photoexcited holes are extremely important to preventing photovoltaic surface corrosion. From the transient absorption analysis, we observed a fast hole transfer from the $2 \mathrm{~S}$ hole level of CdSe SCNC to the HOMO level of PDTC ligand. Therefore, appropriate ligand passivation of both QDs and SCNCs will provide a better understanding of the interfacial hole transfer dynamics, which will allow us to enhance the long-term stability of photovoltaics.

(2) Capturing hot electrons before they relax to the band edge ("electron cooling") can increase the efficiency of photovoltaic devices.[246] The positive signal observed in the long-wavelength region is not only size dependent but also influenced by pump photon energy. Absorption of $388 \mathrm{~nm}$ photons populate the conduction band of SCNCs with hot electrons[247, 248], which are transferred to the interfacial states before relaxing to the conduction band edge because of the strong interfacial electronic interaction between the CdSe SCNC and PDTC. Extraction of hot electrons will likely outpace intraband electron cooling, which has a sub-picosecond time constant[249]. This is important for enhancement of the efficiency in photovoltaic applications.

(3) Because of the large electronic level spacing, (couple of hundred meV, as has been calculated in the literature[232, 250,251], as well as determined by the measured TA peak wavelengths), the hot carrier cooling of our strongly confined $1.6 \mathrm{~nm} \mathrm{CdSe} \mathrm{SCNCs} \mathrm{is}$ much slower than in QDs[216], which makes possible the observation of hole transfer and hot electron transfer signals in the TA spectrum. The carrier cooling phenomenon is strongly 
correlated with multiple exciton generation (MEG)[29, 41, 42] and if the cooling rate is slower than the MEG rate, one will be able to generate a large photocurrent in SCNC-based photovoltaic cells[252].

Taken together, we believe that passivating the surface of ultrasmall SCNCs with highly conjugated organic ligands containing various chemical substituents that both participate in strong electronic coupling and create a larger number of interfacial hybrid orbitals will provide better control over the manipulation of rates of hot electron and hole transfer, intraband cooling, and carrier recombination and thus facilitate the fabrication of highly efficient photovoltaic devices. 


\title{
CHAPTER 4
}

\section{CdSe NANOCLUSTERS PASSIVATED WITH PARA-SUBSTITUTED DERIVATIVES OF PHENYLDITHIOCARBAMATE LIGANDS}

\begin{abstract}
Research described in this chapter is a natural continuation of the ultrafast spectroscopy investigation of the photo-induced dynamics in CdSe nanoclusterphenyldithiocarbamate ligand complexes. The main focus of this research project is to study the energy level alignment between $1.6 \mathrm{~nm} \mathrm{CdSe} \mathrm{nanocluster} \mathrm{core} \mathrm{and} \mathrm{its} \mathrm{hole-delocalizing}$ para-substituted phenyldithiocarbamate derivative (X-PDTC) ligands, and its effect on ultrafast dynamics including charge carrier relaxation, transfer, and recombination. Following discussion in the previous chapter, we compare the state filling signals, as well as photo-induced absorption signals by interfacial charge transfer states, of CdSe nanoclusters conjugated to six X-PDTC ligands with six different functional groups $\left(-\mathrm{CH}_{3}\right.$, $-\mathrm{H},-\mathrm{F},-\mathrm{Br},-\mathrm{OCF}_{3}$, and $\left.-\mathrm{CF}_{3}\right)$ at the para-position of the benzene ring. Because of their difference in electron donating or withdrawing abilities, the impacts of these ligands on the hot electron and hole transfer processes vary. A comparative study of charge transfer across the ligand-nanocluster interfaces is important for improving efficiencies of nanocrystalbased photovoltaic and light-emitting devices that incorporates para-substituted PDTC ligands.
\end{abstract}




\subsection{Introduction}

Semiconductor nanocrystals, or quantum dots (QDs), are ideal building blocks in nanotechnology thanks to high selectivity and tunability of their sizes and shapes $[213,253-$ 256]. In particular, size-dependent optical and electronic properties of QDs enable wide varieties of photovoltaic, optoelectronic, and electronic applications [257-259]. Such sizedependency arises from the quantum confinement effect on the excitons as the diameter of a semiconductor nanoparticle approaches its Bohr radius [260, 261]. Therefore, the most common approach for controlling the optical properties of a semiconductor material is by variation of its size and shape, as in the case of quantum dots, nanorods, nanowires, nanoplatelets, and nanosheets.

Recently, an emerging method for controlling QDs optical properties (such as the absorption profile and photoluminescence quantum yield) through the control of the surface chemistry has drawn great attentions [262]. Quantum dots with sizes ranging from $2-10$ $\mathrm{nm}$ have a high surface-to-core atoms ratio. Therefore, the surface chemistry of the material begins to dominate the properties (such as solubility, optical properties, and charge transfer dynamics) of the entire particle. This effect is especially important for semiconductor nanoclusters with a diameter that is less than $2 \mathrm{~nm}$, and hence $80 \%$ or more atoms are on the surface [230]. Researchers have been using inorganic shells such as ZnS, CdS and CdTe $[64,263,264]$ or organic ligands $[53,265,266]$ to passivate the surface atoms. Surface passivation can modulate the optical properties of the QDs and prevent charge trapping states due to defects, which sabotage the efficiencies of photovoltaic devices [267, 268].

There has been a focus on the ability of certain organic ligands that either withdraw electrons from or donate electrons to the inorganic core structures. This electron 
withdrawing or donating effect is attributed to the conjugation structures, the aromaticity, and the functional groups of the ligand molecules [269, 270]. Ligands with aromatic rings (such as thiophenol [53], aniline [54], phenylenediamine [49, 55]) are especially interesting due to their pi-conjugation structures which offer the strong ability to extract photongenerated charge carriers from the QDs core to the passivated ligands.

Studies have shown that these aromatic molecules are bound to the QDs surface via coordinate covalent bond, which affects both the lifetime and the quantum yield of the photoluminescence (PL) spectra [54]. Enhanced stability against oxidation has also been reported for the ligand-passivated QDs. This stability is mainly attributed to the hole transfer from the QD core to the passivating ligands, and to the delocalization of the molecular orbitals on the aromatic ring [270]. Furthermore, the conjugated structure of the aromatic ring can potentially facilitate charge transfer from QDs to a metal-oxide substrate such as $\mathrm{TiO}_{2}$, which is commonly used in solar devices as the electron conductor in dye-sensitized solar cells [271].

It has been reported that substitution of covalently bounded ligands, such as thiophenol [53], aniline [54, 272], phenylenediamine [49, 55], and phenyldithiocarbamate (PDTC) [226, 243, 273, 274], for native ligands, such as trioctylphosphine oxide (TOPO), trioctyl-phosphine (TOP), tetradecylphosphonic acid (TDPA), or hexadecylamine (HDA), via ligand exchange reactions brought about favorable changes to the optical properties and stabilities of QDs. Especially, studies by Weiss group revealed a decrease of the optical band gap of CdSe QDs when passivated with PDTC ligands [57], which indicates relaxation of the quantum confinement. Based on density functional calculations, this relaxation is most 
likely attributed to the hole wave function delocalization from the QDs surface to the PDTC ligand shell $[57,58]$.

They also found that this modulation of band gaps of ligand-passivated QDs is directly related to the energy level alignment between the QD and the ligands [56]. Based on the Hammett coefficients [275], (i.e. the electron withdrawing and donating ability), and the molecular orbitals of the para-substituents, Weiss and coworkers estimated the HOMO energies of several X-PDTC molecules (Figure 4.1.a). They suggested that due to the energy level alignments between these HOMO levels of PDTCs and the valence band edge of the CdSe QDs, the increase in the effective excitonic radius of the QDs has a linear relation with the HOMO energies of the X-PDCT molecules, with the exception of F-PDTC (Figure 4.1.b).

When Sardar and coworkers [226] used various X-PDTCs to modulate the optical properties of CdSe nanoclusters, an unprecedentedly large and controllable decrease in the optical band gap was observed. Compared to the CdSe QDs $(2-10 \mathrm{~nm})$, variation of the surface chemistry of this $1.6 \mathrm{~nm}$ ultrasmall nanoclusters $\left(\mathrm{Cd}_{34} \mathrm{Se}_{34}\right)$ has a larger influence on the effective excitonic radius. Using ligand molecules such as para-substituted PDTCs [226] and metal-carboxylates [276], the exciton delocalization of this "magic-sized" molecule-like CdSe nanocluster is further studied. These studies revealed that electron-accepting group facilitates the hole transfer processes.[226] 

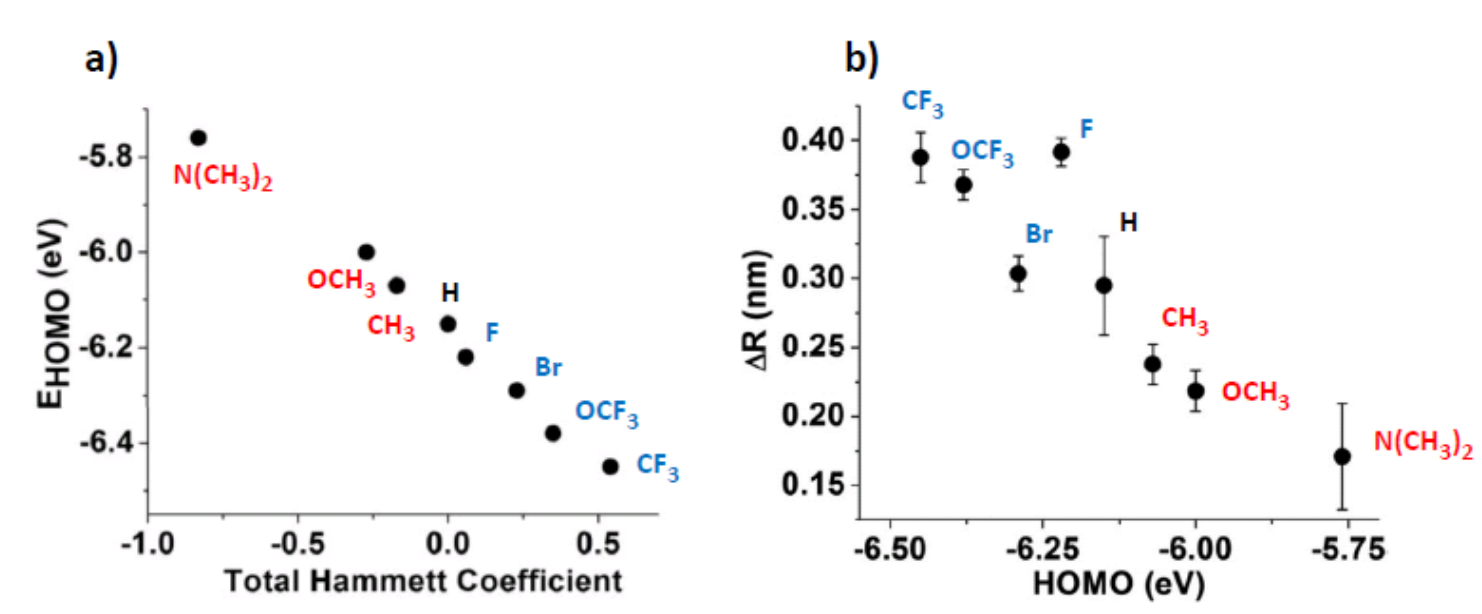

Figure 4.1. (a) HOMO Energy of X-PDTC Ligands against the Hammett Coefficient of the Functional Groups; (b) Change in Excitonic Radius against HOMO Energy.

Figures adapted with permission from reference 56. Copyrights 2012 American Chemistry Society.

Because hole transfer is one of the main factors that limit device efficiency [277, 278], its study may play an important role in the development of QD solar cells. There are still some unexplained observations and debate [279] on whether electron-donating group or electron-withdrawing group facilitates the charge transfer processes. Weiss et al. reconciled this disagreement by suggesting that the inconsistency of the excitonic radius may be attributed to the unbound ligands in the QDs solution due to the ligand degradation process [280]. They also discussed a longstanding puzzle of the large variability of the excitonic radius for the same ligand-passivated QDs in a recent paper [281]. This variability is attributed to the fact that even relatively small changes in the ligand surface coverage, may lead to a measurable effect on the excitonic radius. Reduction of a few percent of bound ligands due to either degradation during storage or the loss of ligand binding sites during particle growth can have a large impact on the QDs band gap. 
Most previous studies on the effect of X-PDTC ligands on charge transfer employs either steady-state absorption or PL emission spectroscopy. Direct measurement of charge carrier dynamics replies on time-resolved spectroscopy investigations. Particularly, femtosecond transient absorption spectroscopy was used in our investigation of photoinduced charge carrier dynamics in $1.6 \mathrm{~nm}$ CdSe QD and X-PDTC complexes, which provides insights into sub-picosecond hot electron and hole transfer processes across the core-ligand interface. The electron withdrawing and donating abilities of the parasubstituted function group are explained in Section 4.1.1. The Transient absorption spectra were analyzed using Hamlett coefficients and the HOMO energy of the X-PDTC molecules, which are shown in Table 4.1.

Table 4.1. Hammett Coefficients $(\sigma)$ and HOMO Energies of Function Groups ${ }^{\mathrm{a}}$

\begin{tabular}{|c|c|c|c|}
\hline $\begin{array}{c}\text { Functional } \\
\text { Group }\end{array}$ & $\sigma_{\text {meta }}$ & $\sigma_{\text {para }}$ & $\begin{array}{c}\text { HOMO Energy } \\
(\mathrm{eV})\end{array}$ \\
\hline$-\mathrm{CH}_{3}$ & -0.069 & -0.17 & -6.08 \\
\hline$-\mathrm{H}$ & 0 & 0 & -6.15 \\
\hline$-\mathrm{F}$ & 0.34 & 0.062 & -6.21 \\
\hline$-\mathrm{Br}$ & 0.39 & 0.23 & -6.30 \\
\hline$-\mathrm{OCF}_{3}$ & 0.38 & 0.35 & -6.38 \\
\hline$-\mathrm{CF}_{3}$ & 0.43 & 0.54 & -6.45 \\
\hline
\end{tabular}

a. Hammett coefficients are adapted with permission from reference 275. Copyrights 1991 American Chemistry Society. HOMO energy adapted from reference 56. Copyrights 2012 American Chemistry Society. 


\subsubsection{Electron Withdrawing/Donating Effect}

The electron withdrawing/donating ability of a functional group is a combining effect of the induction due to electronegativity, and the resonance structures due to hyperconjugation. Hyper-conjugation is the interaction of electrons between a sigma bond (such as $\mathrm{C}-\mathrm{H}$ or $\mathrm{C}-\mathrm{C}$ ) and its adjacent empty (or partially filled) non-bonding p-orbital. Hyperconjugation provides extended molecular orbitals which increases the stability of the system.[282]

Upon methyl substitution of PDTC, because carbon (electronegativity $=2.5$ ) is slightly more electronegative than hydrogen (electronegativity $=2.2$ ), there is a small dipole moment pulling the electron density away from hydrogens of the methyl group, and "donating" the electron density toward the carbon of the methyl group (Figure 4.2.a). This slight build-up of the electron density on the carbon gives the methyl group an electron donating effect by induction. The electron donating effect is stronger on the para position than the meta position due to hyper-conjugation, a special type of resonance effect, resulting in additional electron donating effect for methyl substitution at the para position (Figure

\section{2.b).}

Replacing hydrogen atoms on the methyl group by the much more electronegative fluorine atoms (electronegativity $=4.0$ ), results in an electron withdrawing effect by induction (Figure 4.2.c). Furthermore, same as para-methyl substitution, the hyperconjugation resonance structures lead to an additional electron withdrawing power of the para-trifluoromethyl (-CF3) group (Figure 4.2.d). 


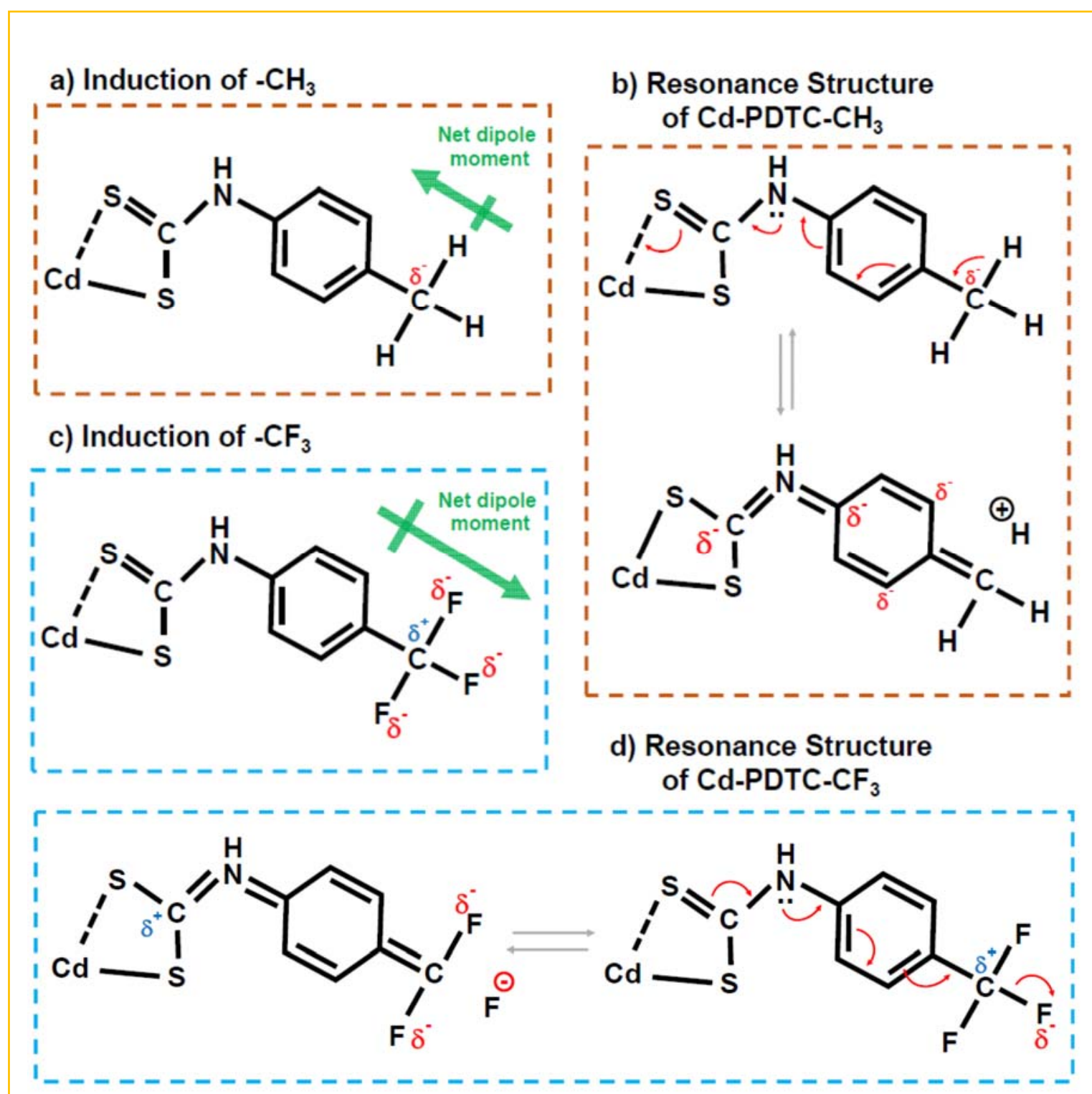

Figure 4.2. Schemetic Electron Densities of Methyl- and Trifluoromethyl- PDTCs

Green arrow describes the direction of the dipole moment, while red arrow indicates the movement of the electrons. Red $\delta^{-}$indicates electron-denser, blue $\delta^{+}$indicates electron-lighter.

For the methyl group $\left(-\mathrm{CH}_{3}\right)$ and trifluoromethyl $\left(-\mathrm{CF}_{3}\right)$, the resonance effect enhanced the induction effect, resulting stronger electron donating or withdrawing ability of the ligands on the para-position. For the other three function groups studied in the current 
project, namely, $-\mathrm{F},-\mathrm{Br}$, and $-\mathrm{OCF}_{3}$, the electronegativity of the halogen atoms results an electron withdrawing effect through induction (Figure 4.3.a). However, the extra lone pair electrons on the $\mathrm{F}, \mathrm{Br}$ or $\mathrm{O}$ atoms, which is directly connected to the benzene ring, put more electron density to the para-position through hyper-conjugation, i.e., provide an electron donating effect (Figure 4.3.b). In this case, the electron withdrawing and donating effects induced by induction and resonance, respectively, are opposite. Even the net effect of these three functional groups are still electron-withdrawing, the Carbon atom at the para-position and the p-orbitals of the dithiol group are slightly electron-denser (Figure 4.3.c).

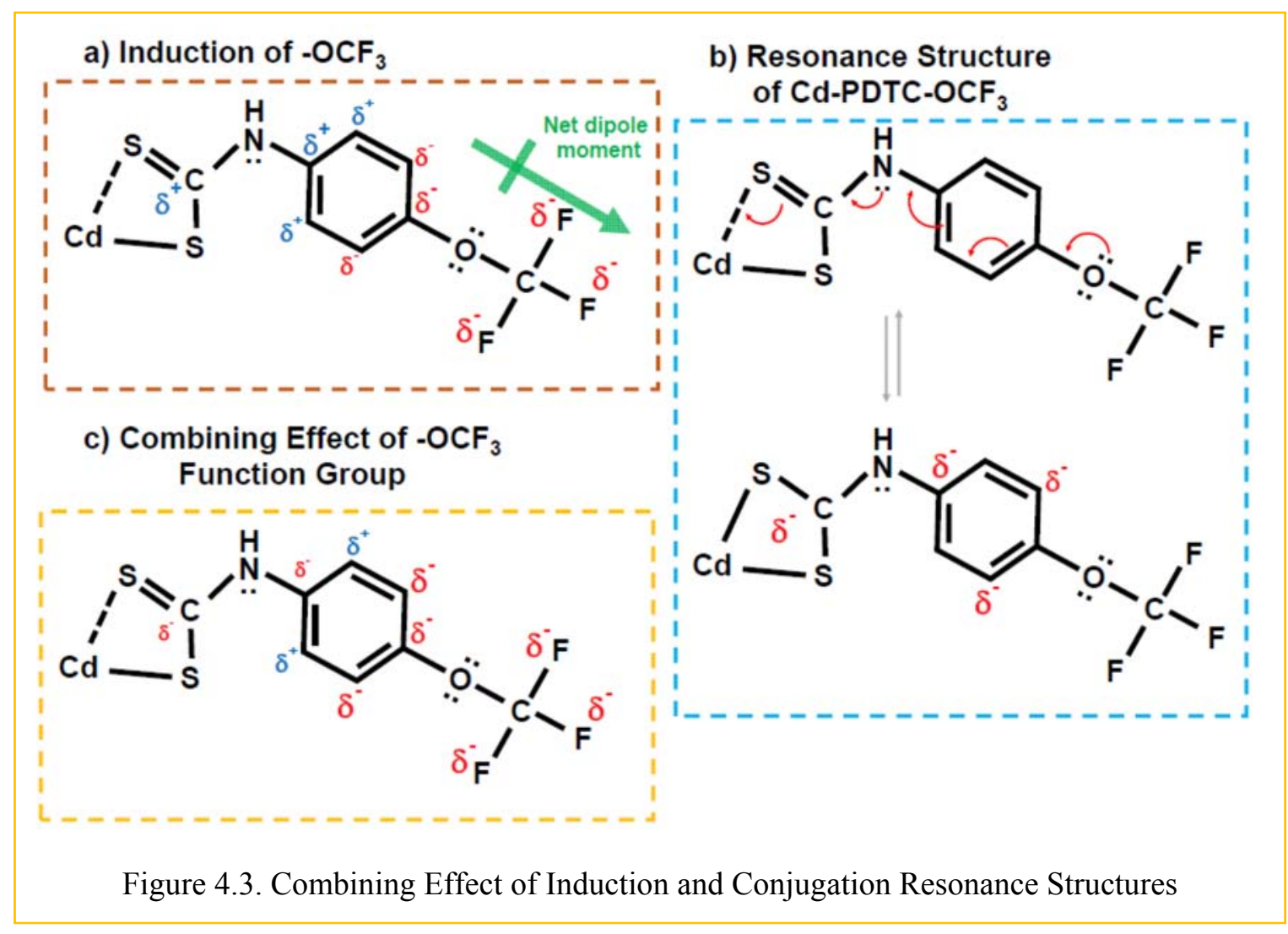




\subsection{Experimental details}

Sample Preparation and Handling. The X-PDTC passivated CdSe nanoclusters used in this work, were generously provided by our collaborators, Dr Rajesh Sardar and coworkers, at IUPUI. Synthesized X-PDTC CdSe nanoclusters were dried and contained in tightly-capped $10 \mathrm{~mL}$ glass tubes. These tubes were completely wrapped by aluminum foil to prevent any types of light exposure. Within 10 days after the synthesis and purification, the tubes were sent through FedEx and placed in a $4{ }^{\circ} \mathrm{C}$ refrigerator immediately upon arrival at our laser facility. When the ultrafast laser system was optimized, CdSe samples (in the form of dry power) were dissolved in DCM. Quick sonication (less than 1 minute) was used to disperse the sample homogenously. After the sample dispersed in DCM, several measurements with different laser setups were taken in the time frame of less than a week, to insure the majority of the PDTC ligands have not degraded and fallen off from the CdSe surface. CdSe NCs that passivated with less stable ligands such as $\mathrm{CF}_{3}-\mathrm{PDTC}$ were studied at first priority, while relatively stable ligands such as Br-PDTC are studied later. F-PDTC CdSe and $\mathrm{CH}_{3}$-PDTC CdSe were synthesized 5 months prior to the synthesis of other $\mathrm{CdSe}$ nanoclusters $\left(\mathrm{OCH}_{3}-\mathrm{PDTC}\right.$, H-PDTC, Br-PDTC and $\left.\mathrm{CF}_{3}-\mathrm{PDTC}\right)$, a slight batch-by-batch discrepancy is suspected between these two batches. The synthesis procedure is published in Langmuir [226] and reprinted below with slight modifications for the reader's convenience. $^{2}$

Synthesis of CdSe Nanoclusters. CdSe NCs (1.6 nm in diameter) were synthesized by our collaborators [226], using their previously published procedures [230]. Briefly, in a $100 \mathrm{~mL}$ two necked round-bottomed flask, $0.140 \mathrm{~g}$ of $\mathrm{CdCl}_{2}$ was mixed with $5 \mathrm{~mL}$ of DDA

\footnotetext{
${ }^{2}$ Permission for reprint is granted, see Appendix I.
} 
at $30{ }^{\circ} \mathrm{C}$ until all solid dissolved. The optically clear solution was cooled to room temperature, and $10 \mathrm{~mL}$ of purged toluene was added before the addition of the selenium precursor. The selenium precursor was prepared separately by dissolving $0.06 \mathrm{~g}$ of freshly grounded selenium powder in a mixture of $785 \mu \mathrm{L}$ of DDA and $215 \mu \mathrm{L}$ of 1-hexanethiol at $30{ }^{\circ} \mathrm{C}$ under $\mathrm{N}_{2}$. After the addition of the selenium precursor, the reaction was stirred under $\mathrm{N}_{2}$ at $40{ }^{\circ} \mathrm{C}$ for $2 \mathrm{~h}$. After $2 \mathrm{~h}$, a stable absorption peak was observed, and the nanocrystals were precipitated by the dropwise addition of purged $\mathrm{MeCN}$. The yellow solid was collected by centrifugation. The precipitation step was repeated two more times. Finally, the solid was dried with N2 and stored in the glove box for further optical characterization and surface modification.

Synthesis of Phenyldithiocarbamate (PDTC) Ligands. Various para-substituted phenyldithiocarbamate (X-PDTC) ligands were prepared according to literature procedures [230]. Briefly, $82.0 \mathrm{mmol}$ of CS2 was added dropwise over $30 \mathrm{~min}$ to $41.0 \mathrm{mmol}$ of the appropriate aniline derivative dispersed in $30 \mathrm{~mL}$ of concentrated $\mathrm{NH}_{4} \mathrm{OH}$ at $0{ }^{\circ} \mathrm{C}$. The solution was stirred under $\mathrm{N}_{2}$ overnight. The resulting products for each aniline derivative appeared as a suspended solid that ranged in color from yellow to white to gray. These products were washed with cold chloroform and dried under vacuum overnight. The 4trifluoromethyl derivative $\left(\mathrm{CF}_{3}\right.$-PDTC) did not precipitate at $0{ }^{\circ} \mathrm{C}$. In order to obtain the solid, the reaction mixture was placed in a freezer overnight until the product crystallized. The X-PDTC ligands were stored in the dark before use.

Ligand Exchange Reaction. All samples were prepared inside a nitrogen-filled glovebox, and reactions were carried out on a Schlenk line. DDA-coated CdSe NC (0.19 mmol) were dissolved in $10 \mathrm{~mL}$ of DCM in a $25 \mathrm{~mL}$ two-necked round-bottomed flask 
followed by the addition of $0.39 \mathrm{mmol}$ of X-PDTC ligand. Since X-PDTC ligands were not soluble in DCM, the biphasic reaction mixture was vigorously stirred at room temperature from 4 to $48 \mathrm{~h}$ depending on the ligand used for the exchange reaction. The exchange reaction was monitored by removing $200 \mu \mathrm{L}$ of the reaction mixture and then centrifuging to remove insoluble solid. The colored solution $(100 \mu \mathrm{L})$ was then diluted in $3 \mathrm{~mL}$ of DCM for $\mathrm{UV}$-vis spectroscopic characterization. After the reaction mixture displayed its stable lowest-energy absorption maximum, it was centrifuged, dried under reduced pressure, and stored inside a glovebox. The X-PDTC-coated samples were stable for at least a month inside the glovebox. The X-PDTC-coated CdSe NCs were completely soluble in DCM.

\subsection{Results and Discussions}

\subsubsection{Wavelength Domain of the TA Spectra}

Compared to CdSe passivated by dodecylamine (DDA), band-edge absorption of XPDTC-passivated CdSe QDs [56] and nanoclusters [226] are red-shifted because of better energy level alignment and hence stronger interaction between the CdSe core and the PDTC ligand layer. In accordance with the previous discussion on the electron donation/withdraw effects, the magnitude of this red shift is dependent on the HOMO level of the ligands, with $-\mathrm{CF}_{3}$ having the lowest HOMO level and the largest shift. TA spectra of X-PDTC-passivated CdSe NCs reveal the same trend. As shown in Figure 4.4, the strong negative peak in the TA spectra is attributed to the state filling effect. Its position corresponds to the $1 \mathrm{~S}(\mathrm{e})-1 \mathrm{~S}_{1 / 2}(\mathrm{~h})$ exciton transition [31].

Of the six functional groups studied in the present TA spectroscopy measurements, methyl-PDTC has the highest HOMO level energy, and weakest delocalization effect [56, 
226]. Therefore, the ground-state absorption of $\mathrm{CH}_{3}$-PDTC passivated $\mathrm{CdSe} \mathrm{NCs}$ has the smallest red-shift from the native NCs [226], and so does the $1 \mathrm{~S}(\mathrm{e})-1 \mathrm{~S}_{1 / 2}(\mathrm{~h})$ exciton transition (the strong negative peak in Figure 4.4), while stronger electron withdrawing groups such as $-\mathrm{Br}$ and $-\mathrm{CF}_{3}$ exhibit larger red-shift.

In addition to the strong $1 \mathrm{~S}(\mathrm{e})-1 \mathrm{~S}_{1 / 2}(\mathrm{~h})$ transition, two other partially resolved negative peaks assigned to $1 \mathrm{~S}(\mathrm{e})-2 \mathrm{~S}_{3 / 2}(\mathrm{~h})$, and $1 \mathrm{~S}(\mathrm{e})-1 \mathrm{~S}_{3 / 2}(\mathrm{~h})$ transitions due to state filling effects were also observed. The peak centers (in $\mathrm{nm}$ ) of these three transitions can be extracted in a fit of the TA spectra to multiple Gaussian functions, and are compared.

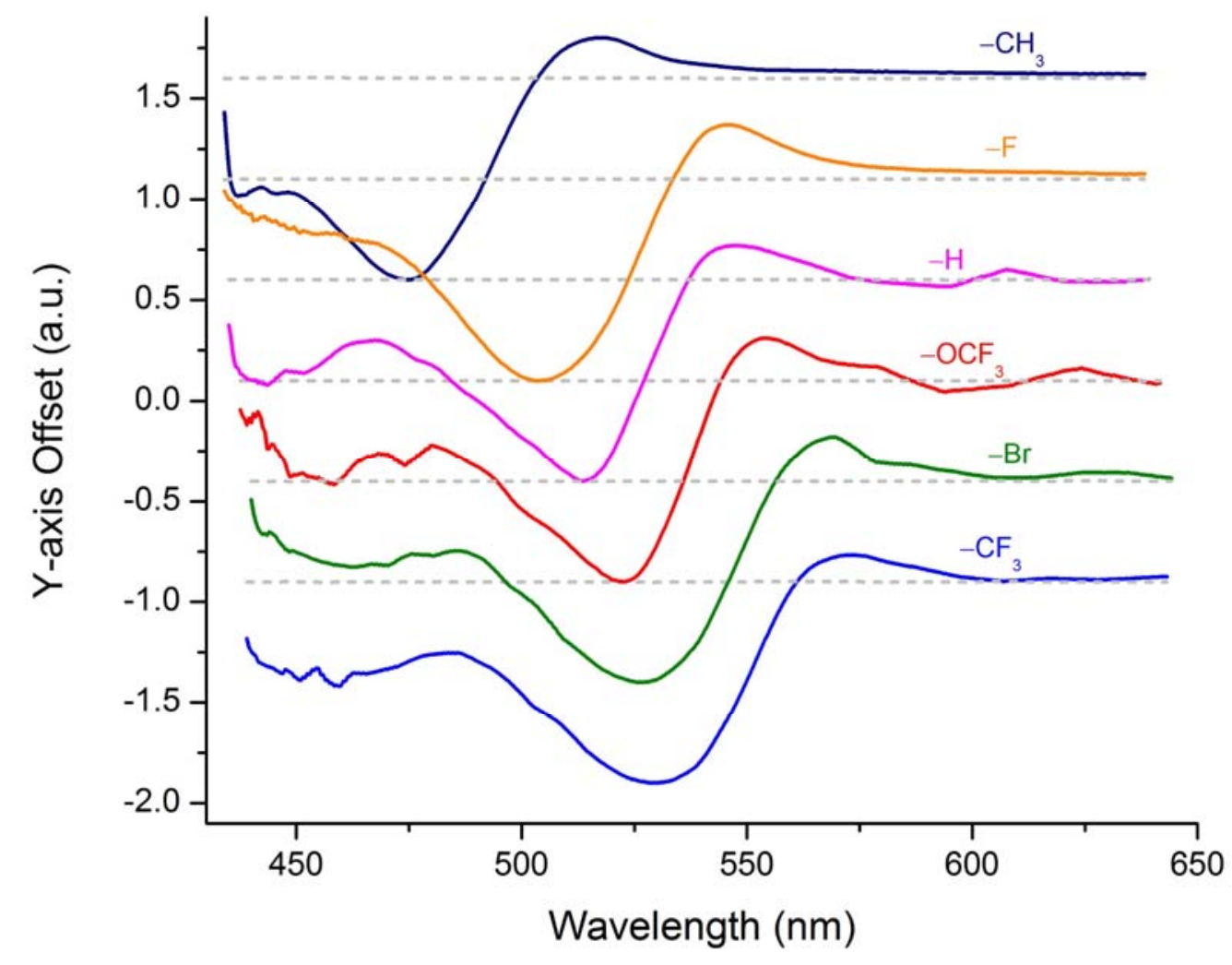

Figure 4.4. Wavelength Domain of TA Spectra of X-PDTC CdSe NCs at $\Delta \mathrm{t}=0.5 \mathrm{ps}$. Spectra are offset in steps of 0.5 a.u. for clarity. Grey dash lines are the baseline levels for each spectrum. 
The red-shifts of all three transitions demonstrate the same trend (Figure 4.5.a) with the steady-state band-edge transition energy that measured by our calibrators [226]. Due to the spectrum lower-limit cut-off at $430 \mathrm{~nm}$, the fitted values of $\lambda_{1}$ (black circle in Figure 4.5.a) of $-\mathrm{CH}_{3},-\mathrm{H}$ and $-\mathrm{F}$ PDTC NCs are likely larger than their true values, hence the energies of $1 \mathrm{~S}(\mathrm{e})-1 \mathrm{~S}_{1 / 2}(\mathrm{~h})$ transition for these three NCs are smaller, resulting a smaller slope of the linear fit $($ slope $=0.54<<1)$.

Regarding the $-\mathrm{CF}_{3}$ and $-\mathrm{OCF}_{3}$ PDTC NCs, we suggest that the low ligand-NCs binding stability of those two X-PDTC NCs is attributed to the smaller red-shifts, therefore they have a large transition energy which are above the liner fitting line. Compared to the steady-state absorption spectra which were taken less than a day after the ligand exchange reactions, the TA spectra were usually taken after $2-4$ weeks. A few percent of relatively unstable ligands, such as $-\mathrm{CF}_{3}$ and $-\mathrm{OCF}_{3}$, were likely detached from the $\mathrm{CdSe}$ core, either during the transportation and storage stages, or the sample handling stage. These few percent of degraded ligands can cause a large decrease of the excitonic radius, i.e., smaller red-shifts, which has been reported for CdSe QDs [283].

When plotting both the excitonic transition energy and the steady-state band-edge energy against the HOMO level of the ligands, a linear relationship is observed (Figure 4.5.b). However, the transition energies obtained from the wavelength domain of the TA spectra exhibit a lower linear correspondence, comparing to the band-edge energy obtained from the steady-state absorption spectra $\left(\mathrm{R}^{2}=0.77\right)$. This is likely attributed by the fact that the latter has only one outliner: $-\mathrm{F}$, while the former has two more outliners: $-\mathrm{CF}_{3}$ and $\mathrm{OCF}_{3}$. 
a)

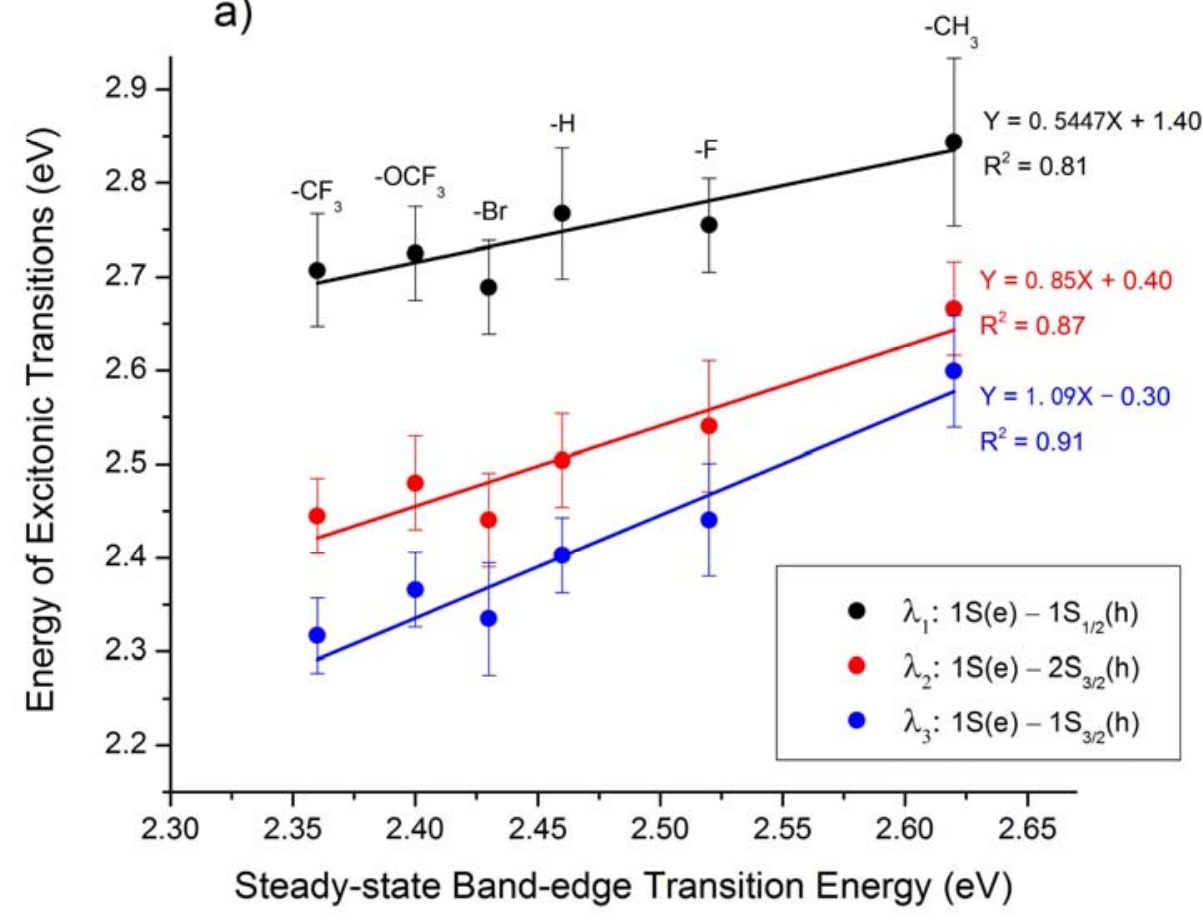

b)

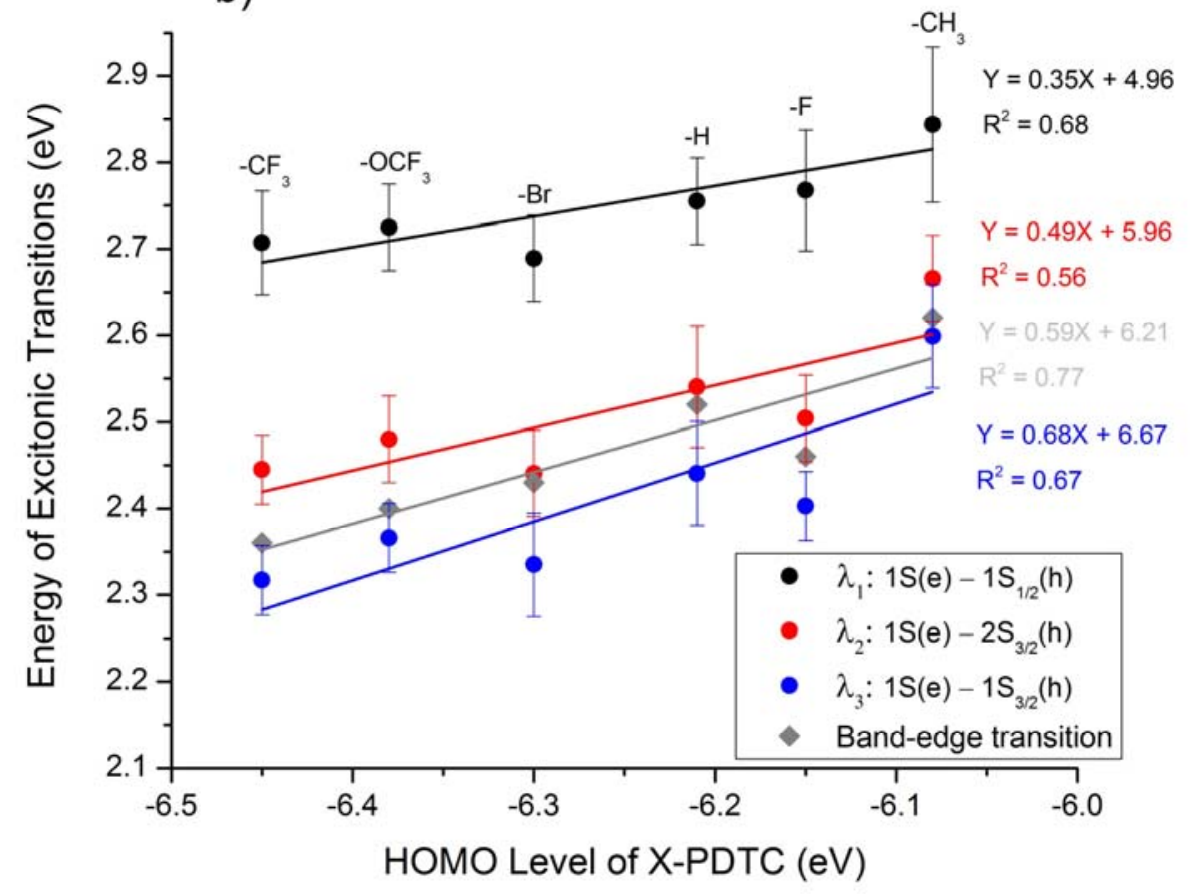

Figure 4.5. Excitonic Transition Energies Are Plotted Against (a) Band-edge Energy of the NCs and (b) HOMO Energy of the Functional Groups. 


\subsubsection{Time Domain of the TA Spectra}

Recovery rate of the negative state-filling signal in the TA spectra is determined by the exciton relaxation and recombination processes. For each NC-ligand complexes, hole relaxation in QDs is significantly faster than electron relaxation because of the higher density of states (DOS) for holes. Normalized transient kinetics traces of the three transitions: $1 \mathrm{~S}(\mathrm{e})-1 \mathrm{~S}_{1 / 2}(\mathrm{~h}), 1 \mathrm{~S}(\mathrm{e})-2 \mathrm{~S}_{3 / 2}(\mathrm{~h})$, and $1 \mathrm{~S}(\mathrm{e})-1 \mathrm{~S}_{3 / 2}(\mathrm{~h})$ ), largely overlap (see Figure 4.6) because all three transitions share the same electron states.

However, in the sub-picosecond regime, difference in the hole transfer rates at the three transitions results different transient kinetics of the state filling signal, which was previously observed in the TA spectra of the H-PDCT CdSe NCs [31]. Except for $\mathrm{CH}_{3}-$ PDTC-passivated NCs, transient kinetics at $1 \mathrm{~S}(\mathrm{e})-2 \mathrm{~S}_{3 / 2}(\mathrm{~h})$ transition $\left(\lambda_{2}\right)$ contains an extra sub-picosecond decay component compared to $1 \mathrm{~S}(\mathrm{e})-1 \mathrm{~S}_{3 / 2}(\mathrm{~h})$ transition $\left(\lambda_{3}\right)$. Furthermore, $1 \mathrm{~S}(\mathrm{e})-1 \mathrm{~S}_{1 / 2}(\mathrm{~h})$ transition $\left(\lambda_{1}\right)$ in $-\mathrm{Br},-\mathrm{F},-\mathrm{OCF}_{3}$, and $-\mathrm{CF}_{3}$ PDTC passivated NCs also contain extra sub-ps decay component.

Thanks to more extended wave function, the $2 \mathrm{~S}_{3 / 2}(\mathrm{~h})$ state is prone to coupling with the HOMO state of the ligands, which utilizes hole transfer. The strength of coupling is determined by the closeness of these two states. The valence band edge of $1.6 \mathrm{~nm} \mathrm{CdSe}$ SCNCs was determined to be $\mathrm{E}_{\mathrm{b}}=-6.16 \mathrm{eV}$. HOMO levels of all X-PTDC ligands studied in the present work are therefore in close proximity to the valence band edge, suggesting strong coupling. However, compared to H-PDTC passivated NCs, the HOMO level of $-\mathrm{Br}$, $-\mathrm{F},-\mathrm{OCF}_{3}$, and $-\mathrm{CF}_{3}$ PDTC passivated $\mathrm{NCs}$ is closer to the $1 \mathrm{~S}_{1 / 2}(\mathrm{~h})$ state, and the electron withdrawing abilities of their functioning group further stabilize the hole delocalization and therefore facilitate hole transfer processes from $2 \mathrm{~S}_{3 / 2}(\mathrm{~h})$ and $1 \mathrm{~S}_{1 / 2}(\mathrm{~h})$ state (Figure 4.7). On 
the other hand, the HOMO level of $-\mathrm{CH}_{3}$ PDTC passivated NCs is farther away from the $1 \mathrm{~S}_{1 / 2}(\mathrm{~h})$ state, and the electron donating group hinder the hole transfer process from NCs.

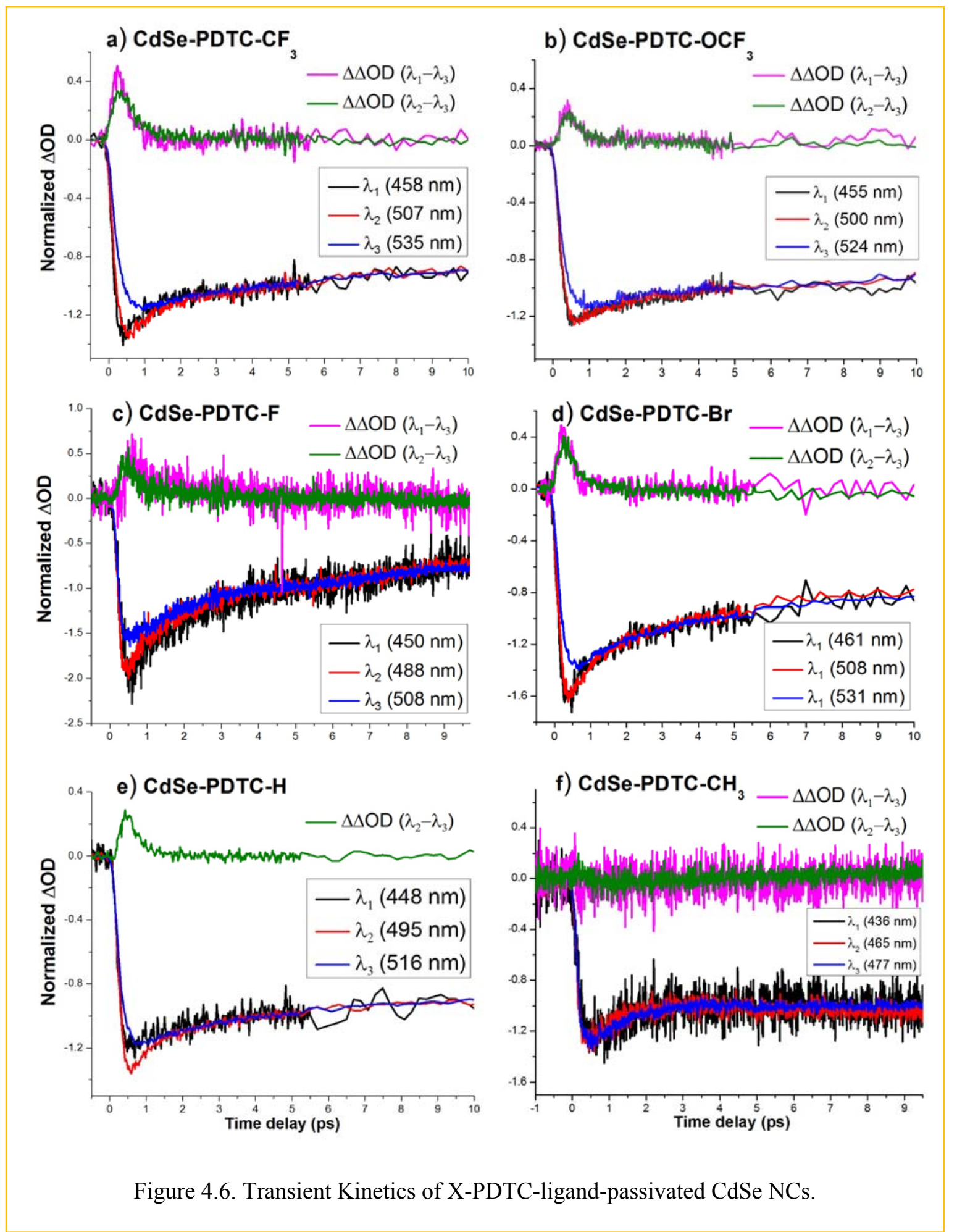




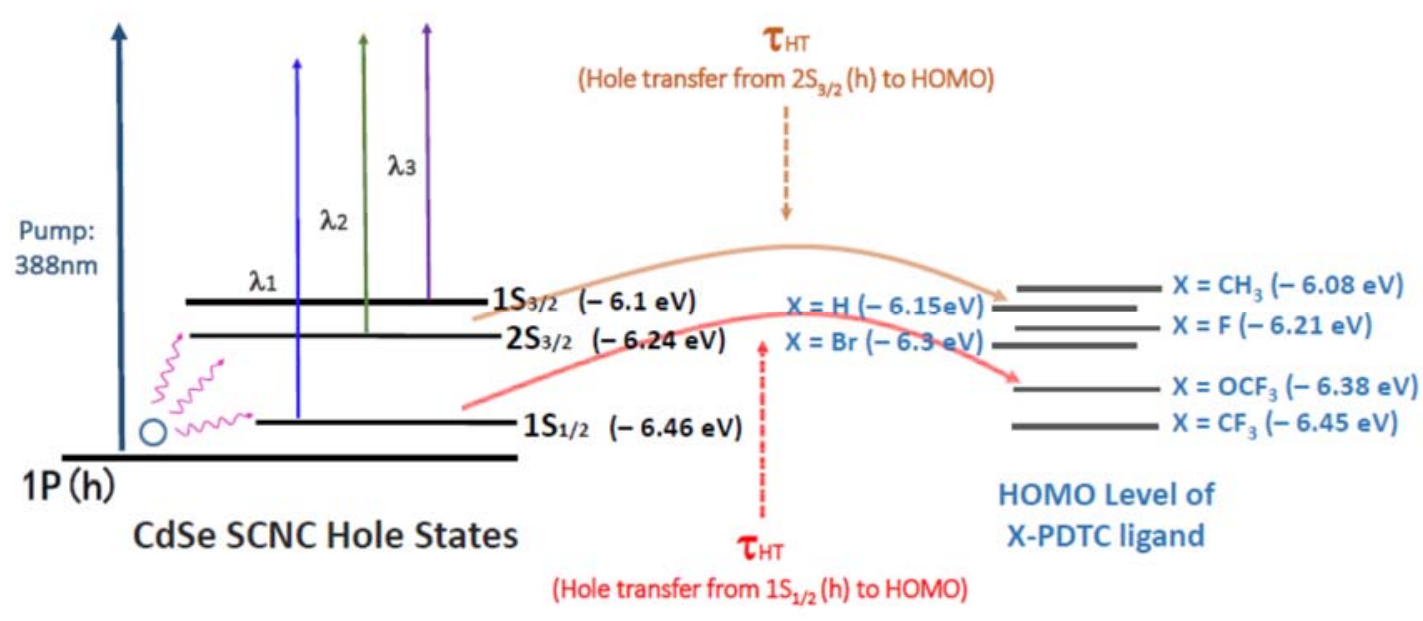

Figure 4.7. Transient Kinetics of X-PDTC-ligand-passivated CdSe NCs.

Time kinetics at the three state filling transitions are normalized. Subtraction is employed to extract the sub-picosecond hole-transfer process.

Such sub-picosecond component can be extracted from the overall transient kinetics by subtraction of normalized $1 \mathrm{~S}(\mathrm{e})-1 \mathrm{~S}_{3 / 2}(\mathrm{~h})\left(\lambda_{3}\right)$ kinetics from that of the $1 \mathrm{~S}(\mathrm{e})-2 \mathrm{~S}_{3 / 2}(\mathrm{~h})\left(\lambda_{2}\right)$ and $1 \mathrm{~S}(\mathrm{e})-1 \mathrm{~S}_{1 / 2}(\mathrm{~h})\left(\lambda_{1}\right)$ transitions. The resulting kinetics are illustrated in Figure 4.6 and fit to an exponential function.

Time constants determined in fitting the sub-picosecond decay curves (Figure 4.8) represent the hole transfer kinetics. According to Macus theory of charge transfer, the charge transfer rate is affected not only by the coupling strength between the initial and the final states, but also the Gibbs free energy of activation. It is therefore generally not true that a stronger coupling leads to faster hole transfer. Although a quantitative explanation of the experimentally determined hole transfer time constants, which summarized in Figure 4.8.f, requires detailed quantum chemical calculations and is beyond the scope of this dissertation, it is worth noting that a simple "particle in a box" model can be used to interpreted the results. If the HOMO level of the ligand is higher or significantly lower than the valence 
band edge, the coupling between the HOMO level and the valence band deepens the potential well, whereas if the HOMO level is lower than the valence band edge, the potential well becomes shallower. Assuming the same coupling strength, the reaction rate increases with a decreasing potential well depth. The experimentally observed hole transfer time constants therefore can be qualitatively explained by the HOMO levels of the passivating ligands.
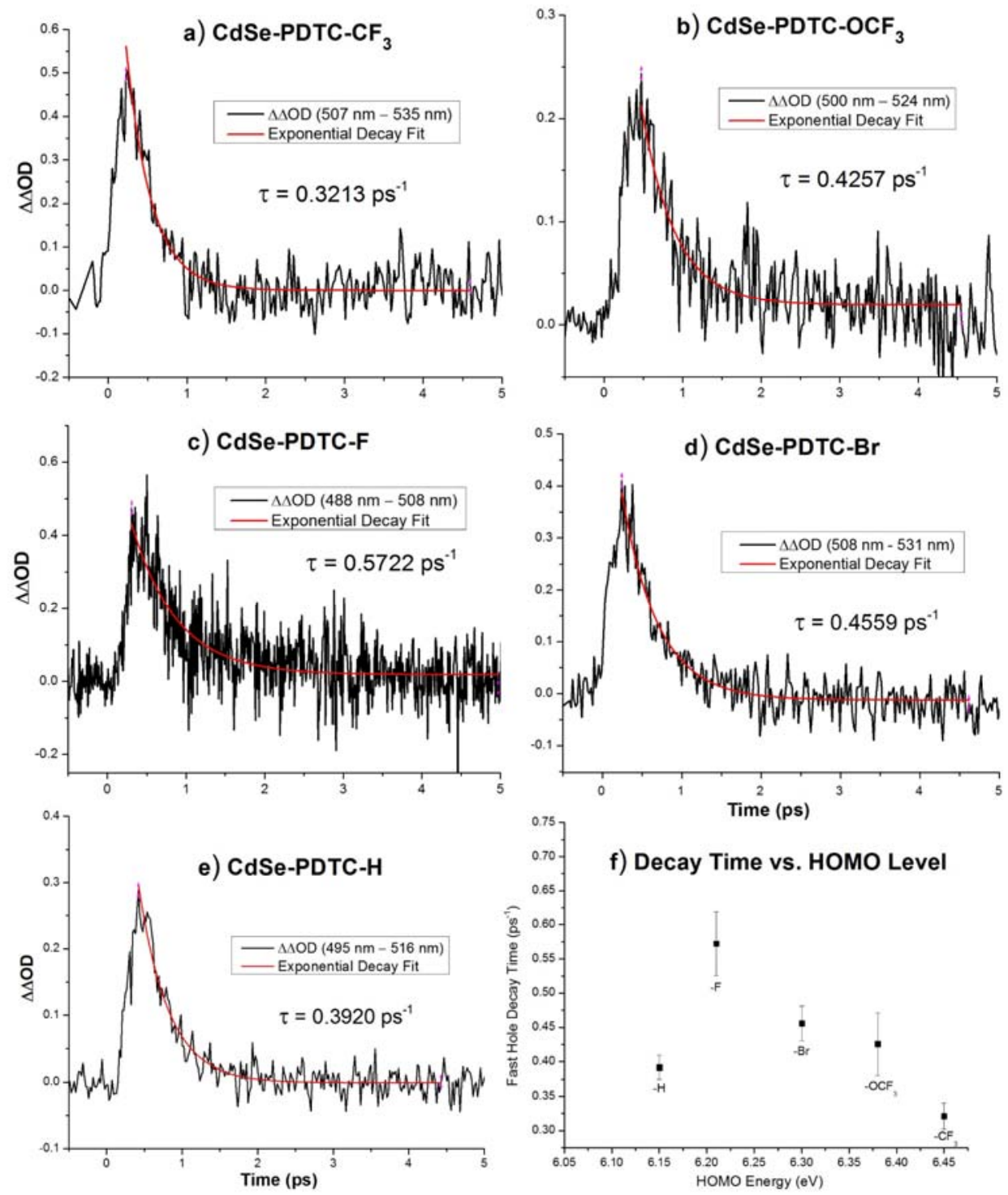

Figure 4.8. (a-e) Transient Kinetics of Hole Transfer form CdSe NCs to X-PDTC Ligands. (f) Time Constants of Hole Transfer plotted against the HOMO Energy. 
The positive sub-picosecond components at $\lambda_{4}$ in all X-PDTC-CdSe NCs are compared in Figure 4.9. Previously, this feature is attributed to photo-induced absorption by the interfacial charge transfer (CT) states [31]. Its rise and decay time therefore correspond to electron transfer from the $\mathrm{NC}$ core to the CT state and from the CT state to the ligand, respectively. Due to the extremely short life time of the CT state, time scale of transient kinetics at $\lambda_{4}$ is expected to be limited by instrument response function and insensitive to the passivation ligands. Such prediction has been confirmed in the TA investigation of X-PDTC-passivated NCs as shown in Figure 4.9, where all traces have the same rising and decaying time, regardless of their X-PDTC ligands.

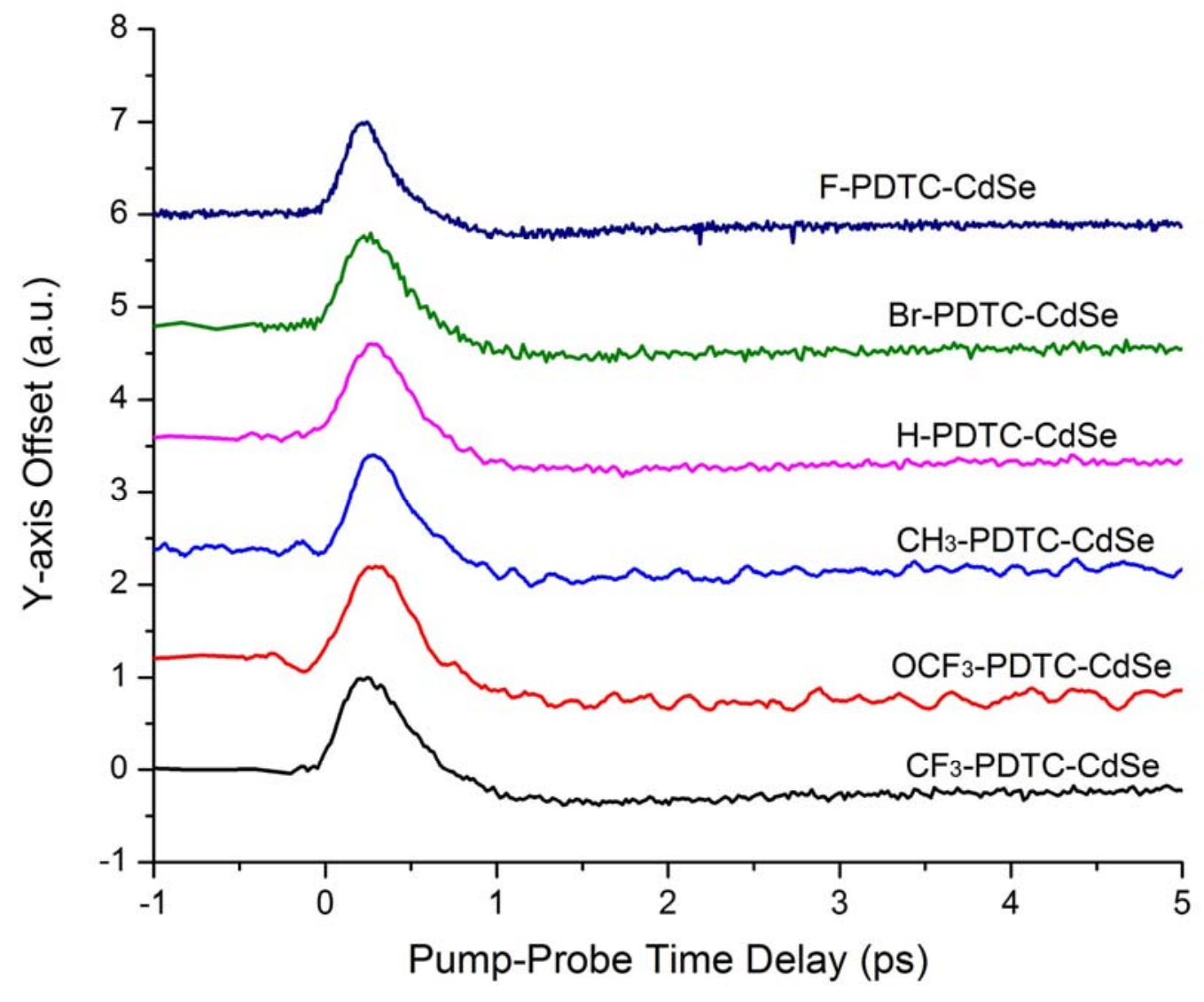

Figure 4.9. Transient Kinetics of Hot Electron Transfer at $\lambda_{4}$. 


\subsection{Conclusions}

TA spectra of CdSe NCs passivated by six para-substituted X-PDTC ligands have been obtained. Comparative studies of the steady-state absorption and the TA spectra (in the frequency domain) of all six complexes unveil correlation between the HOMO levels of the ligands and the excitonic levels of the NC-ligand complexes. Such correlation is attributed to the electron withdrawing/donating effects of the functional groups. Transient kinetics (in the time domain) of ligand-passivated NCs has also been analyzed. The hole transfer rate constants are affected by the ligands while time scale of electron transfer involving the interfacial charge transfer states is insensitive to the ligands but limited mainly by the instrument response function. Experimental observation in this study will help understand the energetics and photo-induced charge carrier dynamics in ligand-passivated NCs, which can be utilized to improve the efficiency of QD-based photovoltaic and other light-emitting devices. 


\section{CHAPTER 5 \\ GLOBAL CONCLUSIONS AND FUTURE DIRECTIONS}

\subsection{Global Conclusion}

Using ultrafast transient absorption (TA) spectroscopy, we studied PDTC ligandpassivated $1.6 \mathrm{~nm}$ diameter CdSe semiconductor nanoclusters (SCNCs) and its parasubstituted X-PDTC derivatives. The TA spectra we obtained unraveled the excitonic dynamics of those ligand-NC conjugates. A fast hole transfer and a hot electron transfer processes were observed, and the globe fitting analysis revealed that both charge transfer

processes are on a sub-picosecond time scale. A series of control experiments was used to demonstrate that the energy level alignment is critical to those sub-picosecond charge transfer processes. Qualitative and comparative analysis on the six para-substituted X-PDTC ligand-passivated $\mathrm{CdSe} \mathrm{NC}$ conjugates reveals the relationship between the HOMO level of ligands and the charge dynamics of the ligand-NC complexes.

Those quantitative and qualitative analysis would deepen our understanding on the kinetics of the charge transfer process across the ligand-NC interface. Knowledge on timecomponents of the ultrafast hole transfer and the electron transfer will help us to better design future photovoltaic devices. Therefore the work presented here have a significant impact on the global energy crisis and renewable energy developments. 


\subsection{Future Directions}

When studying the charge transfer process across ligand-NC interface, the thermal stability of the ligand-NC linkage is critical. Researchers has reported that they observed large variability of the excitonic radius of the PDTC passivated QDs due to the weak binding between the ligand and the semiconductor core [280, 281]. Therefore, increasing the stability of the ligand-NC structure is necessary for further quantitative and accurate analysis. Quantum dots and nanoclusters containing elements such as gold may be future candidates due to the strong binding force of the Au-Sulfur bond which connecting the PDTC ligand and the gold nanoparticles.

Recently, CdSe QDs in the gas phase were successfully prepared and studied by photon electron spectroscopy [284]. This allowed us to study the electronic energy structures of the QDs and the passivating ligands in their isolated forms, while eliminating the effect of the solution and external interactions. Using high-resolution laser spectroscopy techniques, we can investigate the interfacial interactions between the ligand and the QDs in the gas phase, and use ultrafast laser spectroscopy to unravel the charge transfer and energy transfer processes in those isolated complexes. Understanding the charge process in ligand-nanostructure complexes will shine insights on the design and development of novel materials for solar device. The final goal of these studies is to provide experimental and theoretical methods to study the energy dynamics of similar ligand-QDs supramolecular assemblies which could be used in a variety of applications.

Furthermore, with the current setup in the ultrafast laser facility fully functioning, other systems can be investigated using transient absorption spectroscopy with only minor modifications on the pump-probe setup. For example, we can study organic electrolytes that 
used in the standard dye-sensitized solar cells, or conductive polymers that can efficiently transport electrical charge thus having interesting applications in unconventional solar cells and organic LEDs.

Ultrafast transient absorption spectroscopy is a powerful tool in solar energy research, and often results in complicated spectroscopic information of the target system which requires much data analysis. Further advanced software programing would make global fitting more accurate and make data analysis more efficient.

Finally, the current system can be expanded beyond its limit to provide wider wavelength coverage, longer delay time, and better time resolution. The current system covers the wavelength range of $225-1600 \mathrm{~nm}$. Extension of the current coverage into longer wavelength can be accomplished by difference frequency generation using the signal and the idler outputs from the NOPAs. Using $\mathrm{AgGaS}_{2}$ nonlinear crystal and InGaAS photodiode detector, we can expand the wavelength coverage into mid-IR.

Using optical fibers can delay the probe pulse which extend the delay time into nanosecond region, but may cause distortion in signal and is only applicable to singlewavelength source. A better approach for extending the time delay is to do so electronically, using a pulse and delay generator. A hollow fiber compressor can be used to achieve as short as 5 fs pulse duration time, which improves the time resolution of the results. With the aforementioned methods and other techniques such as terahertz spectroscopy, photoinduced absorption spectroscopy, and nanosecond/microsecond pump-probe spectroscopy, the scope of our studies can be significantly expanded. Therefore, more photoinduced materials will be studied until we find the ideal QDs material and structures for high-efficacy and low-cost photovoltaic devices. 


\section{REFERENCES}

1. Gerland, P., Raftery, A.E., Ševčíková, H., Li, N., Gu, D., Spoorenberg, T., Alkema, L., Fosdick, B.K., Chunn, J., Lalic, N., Bay, G., Buettner, T., Heilig, G.K., and Wilmoth, J., World Population Stabilization Unlikely This Century. Science, 2014. 346(6206), 234-237.

2. Lewis, N.S., Crabtree, G., Nozik, A., Wasielewski, M., Alivisatos, P., Basic Research Needs for Solar Energy Utilization. Report of the Basic Energy Sciences Workshop on Solar Energy Utilization, 2005, 1-32.

3. Adamantiades, A. and Kessides, I., Nuclear Power for Sustainable Development: Current Status and Future Prospects. Energy Policy, 2009. 37(12), 5149-5166.

4. Islam, M.T., Shahir, S.A., Uddin, T.M.I., and Saifullah, A.Z.A., Current Energy Scenario and Future Prospect of Renewable Energy in Bangladesh. Renewable Sustainable Energy Rev., 2014. 39, 1074-1088.

5. Union_of_Concerned_Scientists. The Hidden Costs of Fossil Fuels. Available from: http://www.ucsusa.org/clean-energy/coal-and-other-fossil-fuels/hidden-cost-offossils\#bf-toc-4.

6. $\quad$ Gratzel, M., Powering the Planet. Nature, 2000. 403(6768), 363-363.

7. Solar_Power_Authority. A History of Solar Cells: How Technology Has Evolved. Available from: https://www.solarpowerauthority.com/a-history-of-solar-cells/.

8. "April 25, 1954: Bell Labs Demonstrates the First Practical Silicon Solar Cell", in APS News. American Physical Society. 18 (4) April 2009.

9. Wesoff, E., "Sunpower Holds World Record for Most Efficient Rooftop Solar Panel, Again", in Greentech Medium. . June 27, 2016.

10. Nelson, J., The Physics of Solar Cells. 2003, London: Imperial College Press.

11. Honsberg, C. and Bowden, S. Solar Cell Parameters. Available from: http://www.pveducation.org/pvcdrom/iv-curve.

12. Shockley, W. and Queisser, H.J., Detailed Balance Limit of Efficiency of P-N Junction Solar Cells. Journal of Applied Physics, 1961. 32(3), 510-519.

13. Klimov, V.I., Mikhailovsky, A.A., McBranch, D.W., Leatherdale, C.A., and Bawendi, M.G., Quantization of Multiparticle Auger Rates in Semiconductor Quantum Dots. Science, 2000. 287, 1011.

14. Klimov, V.I., Mechanisms for Photogeneration and Recombination of Multiexcitons in Semiconductor Nanocrystals: Implications for Lasing and Solar Energy Conversion. The Journal of Physical Chemistry B, 2006. 110(34), 16827-16845.

15. O'Regan, B. and Gratzel, M., A Low-Cost, High-Efficiency Solar Cell Based on DyeSensitized Colloidal Tio2 Films. Nature, 1991. 353, 737-740.

16. Gratzel, M., Photoelectrochemical Cells. Nature, 2001. 414(6861), 338-344.

17. Efros, A.L. and Efros, A.L., Interband Absorption of Light in a Semiconductor Sphere. Sov. Phys. Semicond., 1982. 16, 772-775. 
18. Brus, L.E., A Simple Model for the Ionization Potential, Electron Affinity, and Aqueous Redox Potentials of Small Semiconductor Crystallites. J. Chem. Phys., 1983. 79, 5566.

19. Jasieniak, J., Califano, M., and Watkins, S.E., Size-Dependent Valence and Conduction Band-Edge Energies of Semiconductor Nanocrystals. ACS Nano, 2011. 5,5888 .

20. Moreels, I., Justo, Y., De Geyter, B., Haustraete, K., Martins, J.C., and Hens, Z., Size-Tunable, Bright, and Stable Pbs Quantum Dots: A Surface Chemistry Study. ACS Nano, 2011. 5, 2004.

21. Moreels, I., Lambert, K., Smeets, D., De Muynck, D., Nollet, T., Martins, J.C., Vanhaecke, F., Vantomme, A., Delerue, C., and Allan, G., Size-Dependent Optical Properties of Colloidal Pbs Quantum Dots. ACS Nano, 2009. 3, 3023.

22. Rohde, R.A. Solar Spectrum. 2007; Available from: https://commons.wikimedia.org/wiki/File:Solar_Spectrum.png.

23. Santra, P.K. and Kamat, P.V., Tandem-Layered Quantum Dot Solar Cells: Tuning the Photovoltaic Response with Luminescent Ternary Cadmium Chalcogenides. J. Am. Chem. Soc., 2013. 135, 877.

24. Brus, L., Electronic Wave Functions in Semiconductor Clusters: Experiment and Theory. J. Phys. Chem., 1986. 90, 2555.

25. Yu, W.W., Qu, L., Guo, W., and Peng, X., Experimental Determination of the Extinction Coefficient of Cdte, Cdse, and Cds Nanocrystals. Chem. Mater., 2003. 15, 2854.

26. Murphy, C.J. and Coffer, J.L., Quantum Dots: A Primer. Applied Spectroscopy, 2002. 56(1), 16A-27A.

27. Klimov, V.I., Spectral and Dynamical Properties of Multilexcitons in Semiconductor Nanocrystals. Annu. Rev. Phys. Chem., 2007. 58, 635.

28. Mikhailovsky, A.A., Malko, A.V., Hollingsworth, J.A., Bawendi, M.G., and Klimov, V.I., Multiparticle Interactions and Stimulated Emission in Chemically Synthesized Quantum Dots. Applied Physics Letters, 2002. 80(13), 2380-2382.

29. Klimov, V.I., Mikhailovsky, A.A., Xu, S., Malko, A., Hollingsworth, J.A., Leatherdale, C.A., Eisler, H.J., and Bawendi, M.G., Optical Gain and Stimulated Emission in Nanocrystal Quantum Dots. Science, 2000. 290(5490), 314.

30. Efros, A.L. and Rosen, M., The Electronic Structure of Semiconductor Nanocrystals. Annual Review of Materials Science, 2000. 30(1), 475-521.

31. Xie, Y., Teunis, M.B., Pandit, B., Sardar, R., and Liu, J., Molecule-Like Cdse Nanoclusters Passivated with Strongly Interacting Ligands: Energy Level Alignment and Photoinduced Ultrafast Charge Transfer Processes. The Journal of Physical Chemistry C, 2015. 119(5), 2813-2821.

32. Pivrikas, A., Neugebauer, H., and Sariciftci, N.S., Charge Carrier Lifetime and Recombination in Bulk Heterojunction Solar Cells. IEEE Journal of Selected Topics in Quantum Electronics, 2010. 16(6), 1746-1758.

33. Zhu, H. and Lian, T., Enhanced Multiple Exciton Dissociation from Cdse Quantum Rods: The Effect of Nanocrystal Shape. J. Am. Chem. Soc., 2012. 134, 11289.

34. Grancini, G., Maiuri, M., Fazzi, D., Petrozza, A., Egelhaaf, H.J., Brida, D., Cerullo, G., and Lanzani, G., Hot Exciton Dissociation in Polymer Solar Cells. Nat. Mater., 2013. $12,29$. 
35. Huang, J., Huang, Z.Q., Yang, Y., Zhu, H.M., and Lian, T.Q., Multiple Exciton Dissociation in Cdse Quantum Dots by Ultrafast Electron Transfer to Adsorbed Methylene Blue. J. Am. Chem. Soc., 2010. 132, 4858.

36. Johnston, K.W., Pattantyus-Abraham, A.G., Clifford, J.P., Myrskog, S.H., Hoogland, S., Shukla, H., Klem, E.J.D., Levina, L., and Sargent, E.H., Efficient Schottky-Quantum-Dot Photovoltaics: The Roles of Depletion, Drift, and Diffusion. Appl. Phys. Lett., 2008. 92, 122111.

37. Zhitomirsky, D., Voznyy, O., Hoogland, S., and Sargent, E.H., Measuring Charge Carrier Diffusion in Coupled Colloidal Quantum Dot Solids. ACS Nano, 2013. 7, 5282.

38. Shockley, W. and Read, W.T., Statistics of the Recombinations of Holes and Electrons. Physical Review A: Atomic, Molecular, and Optical Physics, 1952. 87(5), 835-842.

39. Hall, R.N., Germanium Rectifier Characteristics. Physical Review A: Atomic, Molecular, and Optical Physics, 1951. 83(1), 228.

40. Wang, L.W., Califano, M., Zunger, A., and Franceschetti, A., Pseudopotential Theory of Auger Processes in Cdse Quantum Dots. Phys. Rev. Lett., 2003. 91, 056404 .

41. Ellingson, R.J., Beard, M.C., Johnson, J.C., Yu, P., Micic, O.I., Nozik, A.J., Shabaev, A., and Efros, A.L., Highly Efficient Multiple Exciton Generation in Colloidal Pbse and Pbs Quantum Dots. Nano Lett., 2005. 5, 865.

42. Pandey, A. and Guyot-Sionnest, P., Slow Electron Cooling in Colloidal Quantum Dots. Science, 2008. 322, 929.

43. Zhu, H., Yang, Y., Wu, K., and Lian, T., Charge Transfer Dynamics from Photoexcited Semiconductor Quantum Dots. Annual Review of Physical Chemistry, 2016. 67(1), 259-281.

44. Zhu, H., Song, N., Rodríguez-Córdoba, W., and Lian, T., Wave Function Engineering for Efficient Extraction of up to Nineteen Electrons from One Cdse/Cds Quasi-Type Ii Quantum Dot. J. Am. Chem. Soc., 2012. 134, 4250.

45. Zhu, H.M., Yang, Y., and Lian, T.Q., Multiexciton Annihilation and Dissociation in Quantum Confined Semiconductor Nanocrystals. Acc. Chem. Res., 2013. 46, 1270.

46. Yang, Y., Rodríguez-Córdoba, W., and Lian, T., Multiple Exciton Generation and Dissociation in Pbs Quantum Dot-Electron Acceptor Complexes. Nano Letters, 2012. 12(8), 4235-4241.

47. Zhu, H., Yang, Y., Hyeon-Deuk, K., Califano, M., Song, N., Wang, Y., Zhang, W., Prezhdo, O.V., and Lian, T., Auger-Assisted Electron Transfer from Photoexcited Semiconductor Quantum Dots. Nano Letters, 2014. 14(3), 1263-1269.

48. Hines, D.A. and Kamat, P.V., Quantum Dot Surface Chemistry: Ligand Effects and Electron Transfer Reactions. J. Phys. Chem. C., 2013. 117, 14418.

49. Zillner, E., Fengler, S., Niyamakom, P., Rauscher, F., Kohler, K., and Dittrich, T., Role of Ligand Exchange at Cdse Quantum Dot Layers for Charge Separation. J. Phys. Chem. C, 2012. 116, 16747.

50. Peterson, M.D., Cass, L.C., Harris, R.D., Edme, K., Sung, K., and Weiss, E.A., The Role of Ligands in Determining the Exciton Relaxation Dynamics in Semiconductor Quantum Dots. Annu. Rev. Phys. Chem., 2014. 65, 317. 
51. Kilina, S., Velizhanin, K.A., Ivanov, S., Prezhdo, O.V., and Tretiak, S., Surface Ligands Increase Photoexcitation Relaxation Rates in Cdse Quantum Dots. ACS Nano, 2012. 6, 6515.

52. Lokteva, I., Radychev, N., Witt, F., Borchert, H., Parisi, J., and Kolny-Olesiak, J., Surface Treatment of Cdse Nanoparticles for Application in Hybrid Solar Cells: The Effect of Multiple Ligand Exchange with Pyridine. J. Phys. Chem. C, 2010. 114, 12784.

53. Liu, I.S., Lo, H.H., Chien, C.T., Lin, Y.Y., Chen, C.W., Chen, Y.F., Su, W.F., and Liou, S.C., Enhancing Photoluminescence Quenching and Photoelectric Properties of Cdse Quantum Dots with Hole Accepting Ligands. J. Mater. Chem., 2008. 18, 675.

54. Knowles, K.E., Tice, D.B., McArthur, E.A., Solomon, G.C., and Weiss, E.A., Chemical Control of the Photoluminescence of Cdse Quantum Dot-Organic Complexes with a Series of Para-Substituted Aniline Ligands. J. Am. Chem. Soc., 2010. 132, 1041.

55. Sharma, S.N., Pillai, Z.S., and Kamat, P.V., Photoinduced Charge Transfer between Cdse Quantum Dots and P-Phenylenediamine. J. Phys. Chem. B, 2003. 107, 10088.

56. Frederick, M.T., Amin, V.A., Swenson, N.K., Ho, A.Y., and Weiss, E.A., Control of Exciton Confinement in Quantum Dot-Organic Complexes through Energetic Alignment of Interfacial Orbitals. Nano Letters, 2012. 13, 287.

57. Frederick, M.T. and Weiss, E.A., Relaxation of Exciton Confinement in Cdse Quantum Dots by Modification with a Conjugated Dithiocarbamate Ligand. ACS Nano, 2010. 4(6), 3195-3200.

58. Frederick, M.T., Amin, V.A., and Weiss, E.A., Optical Properties of Strongly Coupled Quantum Dot-Ligand Systems. J. Phys. Chem. Lett., 2013. 4, 634.

59. Nozik, A.J., Quantum Dot Solar Cells. Physica E: Low-dimensional Systems and Nanostructures, 2002. 14(1-2), 115-120.

60. Bera, D., Qian, L., Tseng, T.-K., and Holloway, P.H., Quantum Dots and Their Multimodal Applications: A Review. Materials, 2010. 3(4).

61. Ekimov, A.I. and Onushchenko, A.A., Quantum Size Effect in the Optical-Spectra of Semiconductor Micro-Crystals. Sov. Phys. Semicond., 1982. 16, 775.

62. Brus, L.E., Electron-Electron and Electron-Hole Interactions in Small Semiconductor Crystallites: The Size Dependence of the Lowest Excited Electronic State. J. Chem. Phys., 1984. 80, 4403.

63. Murray, C.B., Norris, D.J., and Bawendi, M.G., Synthesis and Characterization of Nearly Monodisperse Cde (E = Sulfur, Selenium, Tellurium) Semiconductor Nanocrystallites. Journal of the American Chemical Society, 1993. 115(19), 87068715.

64. Spanhel, L., Haase, M., Weller, H., and Henglein, A., Photochemistry of Colloidal Semiconductors. 20. Surface Modification and Stability of Strong Luminescing Cds Particles. Journal of the American Chemical Society, 1987. 109(19), 5649-5655.

65. Kortan, A.R., Hull, R., Opila, R.L., Bawendi, M.G., Steigerwald, M.L., Carroll, P.J., and Brus, L.E., Nucleation and Growth of Cadmium Selendie on Zinc Sulfide Quantum Crystallite Seeds, and Vice Versa, in Inverse Micelle Media. Journal of the American Chemical Society, 1990. 112(4), 1327-1332.

66. Grieser, F., Furlong, D.N., Scoberg, D., Ichinose, I., Kimizuka, N., and Kunitake, T., Size-Quantised Semiconductor Cadmium Chalcogenide Particles in Langmuir- 
Blodgett Films. Journal of the Chemical Society, Faraday Transactions, 1992. 88(15), 2207-2214.

67. Alivisatos, A.P., Semiconductor Clusters, Nanocrystals, and Quantum Dots. Science, 1996. 271(5251), 933.

68. Sundaram, M., Chalmers, S.A., Hopkins, P.F., and Gossard, A.C., New Quantum Structures. Science, 1991. 254(5036), 1326.

69. Artemyev, M.V., Woggon, U., Wannemacher, R., Jaschinski, H., and Langbein, W., Light Trapped in a Photonic Dot: Microspheres Act as a Cavity for Quantum Dot Emission. Nano Letters, 2001. 1(6), 309-314.

70. Carey, G.H., Abdelhady, A.L., Ning, Z., Thon, S.M., Bakr, O.M., and Sargent, E.H., Colloidal Quantum Dot Solar Cells. Chemical Reviews, 2015. 115(23), 1273212763.

71. Graham-Rowe, D., From Dots to Devices. Nat Photon, 2009. 3(6), 307-309.

72. Coe-Sullivan, S., Optoelectronics: Quantum Dot Developments. Nat Photon, 2009. 3(6), 315-316.

73. Park, J., Joo, J., Kwon, S.G., Jang, Y., and Hyeon, T., Synthesis of Monodisperse Spherical Nanocrystals. Angew. Chem., Int. Ed., 2007. 46, 4630.

74. Thanh, N.T.K., Maclean, N., and Mahiddine, S., Mechanisms of Nucleation and Growth of Nanoparticles in Solution. Chem. Rev., 2014. 114, 7610.

75. Yin, Y. and Alivisatos, A.P., Colloidal Nanocrystal Synthesis and the OrganicInorganic Interface. Nature, 2005. 437, 664.

76. García-Rodríguez, R., Hendricks, M.P., Cossairt, B.M., Liu, H., and Owen, J.S., Conversion Reactions of Cadmium Chalcogenide Nanocrystal Precursors. Chem. Mater., 2013. 25, 1233.

77. Qu, L. and Peng, X., Control of Photoluminescence Properties of Cdse Nanocrystals in Growth. J. Am. Chem. Soc., 2002. 124, 2049.

78. Burda, C., Chen, X., Narayanan, R., and El-Sayed, M.A., Chemistry and Properties of Nanocrystals of Different Shapes. Chem. Rev., 2005. 105, 1025.

79. Choi, S.-H., Song, H., Park, I.K., Yum, J.-H., Kim, S.-S., Lee, S., and Sung, Y.-E., Synthesis of Size-Controlled Cdse Quantum Dots and Characterization of CdseConjugated Polymer Blends for Hybrid Solar Cells. Journal of Photochemistry and Photobiology A: Chemistry, 2006. 179(1-2), 135-141.

80. Zlateva, G., Zhelev, Z., Bakalova, R., and Kanno, I., Precise Size Control and Synchronized Synthesis of Six Colors of Cdse Quantum Dots in a Slow-Increasing Temperature Gradient. Inorganic Chemistry, 2007. 46(16), 6212-6214.

81. Kwon, S.G., Piao, Y., Park, J., Angappane, S., Jo, Y., Hwang, N.M., Park, J.G., and Hyeon, T., Kinetics of Monodisperse Iron Oxide Nanocrystal Formation by "Heating-up" Process. J. Am. Chem. Soc., 2007. 129, 12571.

82. Newton, J.C., Ramasamy, K., Mandal, M., Joshi, G.K., Kumbhar, A., and Sardar, R., Low-Temperature Synthesis of Magic-Sized Cdse Nanoclusters: Influence of Ligands on Nanocluster Growth and Photophysical Properties. The Journal of Physical Chemistry C, 2012. 116(7), 4380-4389.

83. Wang, Y., Zhang, Y., Wang, F., Giblin, D.E., Hoy, J., Rohrs, H.W., Loomis, R.A., and Buhro, W.E., The Magic-Size Nanocluster (Cdse)34 as a Low-Temperature Nucleant for Cadmium Selenide Nanocrystals; Room-Temperature Growth of Crystalline Quantum Platelets. Chemistry of Materials, 2014. 26(7), 2233-2243. 
84. Nguyen, K.A., Day, P.N., and Pachter, R., Understanding Structural and Optical Properties of Nanoscale Cdse Magic-Size Quantum Dots: Insight from Computational Prediction. J. Phys. Chem. C, 2010. 114, 16197.

85. King, L.A. and Riley, D.J., Importance of Qd Purification Procedure on Surface Adsorbance of Qds and Performance of Qd Sensitized Photoanodes. J. Phys. Chem. C, 2012. 116, 3349.

86. Remacle, F., On Electronic Properties of Assemblies of Quantum Nanodots. J. Phys. Chem. A, 2000. 104, 4739.

87. Talgorn, E., Moysidou, E., Abellon, R.D., Savenije, T.J., Goossens, A., Houtepen, A.J., and Siebbeles, L.D.A., Highly Photoconductive Cdse Quantum-Dot Films: Influence of Capping Molecules and Film Preparation Procedure. J. Phys. Chem. C, 2010. 114, 3441.

88. Sun, L., Choi, J.J., Stachnik, D., Bartnik, A.C., Hyun, B.R., Malliaras, G.G., Hanrath, T., and Wise, F.W., Bright Infrared Quantum-Dot Light-Emitting Diodes through Inter-Dot Spacing Control. Nat. Nanotechnol., 2012. 7, 369.

89. Ip, A.H., Thon, S.M., Hoogland, S., Voznyy, O., Zhitomirsky, D., Debnath, R., Levina, L., Rollny, L.R., Carey, G.H., and Fischer, A., Hybrid Passivated Colloidal Quantum Dot Solids. Nat. Nanotechnol., 2012. 7, 577.

90. Liu, Y., Gibbs, M., Puthussery, J., Gaik, S., Ihly, R., Hillhouse, H.W., and Law, M., Dependence of Carrier Mobility on Nanocrystal Size and Ligand Length in Pbse Nanocrystal Solids. Nano Lett., 2010. 10, 1960.

91. Smith, A.R., Yoon, W., Heuer, W.B., Baril, S.I.M., Boercker, J.E., Tischler, J.G., and Foos, E.E., Effect of Ligand Structure on the Optical and Electronic Properties of Nanocrystalline Pbse Films. J. Phys. Chem. C, 2012. 116, 6031.

92. Zarghami, M.H., Liu, Y., Gibbs, M., Gebremichael, E., Webster, C., and Law, M., P-Type Pbse and Pbs Quantum Dot Solids Prepared with Short-Chain Acids and Diacids. ACS Nano, 2010. 4, 2475.

93. Zhitomirsky, D., Furukawa, M., Tang, J., Stadler, P., Hoogland, S., Voznyy, O., Liu, H., and Sargent, E.H., N-Type Colloidal-Quantum-Dot Solids for Photovoltaics. Adv. Mater., 2012. 24, 6181.

94. Ning, Z., Dong, H., Zhang, Q., Voznyy, O., and Sargent, E.H., Solar Cells Based on Inks of N-Type Colloidal Quantum Dots. ACS Nano, 2014. 8, 10321.

95. Ning, Z., Voznyy, O., Pan, J., Hoogland, S., Adinolfi, V., Xu, J., Li, M., Kirmani, A.R., Sun, J.P., and Minor, J., Air-Stable N-Type Colloidal Quantum Dot Solids. Nat. Mater., 2014. 13, 822.

96. Reiss, P., Protière, M., and Li, L., Core/Shell Semiconductor Nanocrystals. Small, 2009. 5, 154.

97. Neo, M.S., Venkatram, N., Li, G.S., Chin, W.S., and Ji, W., Synthesis of Pbs/Cds Core-Shell Qds and Their Nonlinear Optical Properties. J. Phys. Chem. C, 2010. 114, 18037.

98. Justo, Y., Geiregat, P., Hoecke, K.V., Vanhaecke, F., De Mello Donega, C., and Hens, Z., Optical Properties of Pbs/Cds Core/Shell Quantum Dots. J. Phys. Chem. C, 2013. 117, 20171.

99. Zhao, H., Fan, Z., Liang, H., Selopal, G.S., Gonfa, B.A., Jin, L., Soudi, A., Cui, D., Enrichi, F., and Natile, M.M., Controlling Photoinduced Electron Transfer from 
Pbs@CdsCore@Shell Quantum Dots to Metal Oxide Nanostructured Thin Films. Nanoscale, 2014. 6, 7004.

100. Neo, D.C.J., Cheng, C., Stranks, S.D., Fairclough, S.M., Kim, J.S., Kirkland, A.I., Smith, J.M., Snaith, H.J., Assender, H.E., and Watt, A.A.R., Influence of Shell Thickness and Surface Passivation on Pbs/Cds Core/Shell Colloidal Quantum Dot Solar Cells. Chem. Mater., 2014. 26, 4004.

101. Ivanov, S.A., Piryatinski, A., Nanda, J., Tretiak, S., Zavadil, K.R., Wallace, W.O., Werder, D., and Klimov, V.I., Type-Ii Core/Shell Cds/Znse Nanocrystals: Synthesis, Electronic Structures, and Spectroscopic Properties. J. Am. Chem. Soc., 2007. 129, 11708.

102. Ning, Z., Yuan, C., Tian, H., Fu, Y., Li, L., Sun, L., and Ågren, H., Type-Ii Colloidal Quantum Dot Sensitized Solar Cells with a Thiourea Based Organic Redox Couple. J. Mater. Chem., 2012. 22, 6032.

103. Zhang, Q., Chen, G., Yang, Y., Shen, X., Zhang, Y., Li, C., Yu, R., Luo, Y., Li, D., and Meng, Q., Toward Highly Efficient Cds/Cdse Quantum Dots-Sensitized Solar Cells Incorporating Ordered Photoanodes on Transparent Conductive Substrates. Phys. Chem. Chem. Phys., 2012. 14, 6479.

104. Fischer, A., Rollny, L., Pan, J., Carey, G.H., Thon, S.M., Hoogland, S., Voznyy, O., Zhitomirsky, D., Kim, J.Y., and Bakr, O.M., Directly Deposited Quantum Dot Solids Using a Colloidally Stable Nanoparticle Ink. Adv. Mater., 2013. 25, 5742.

105. Cassagneau, T., Mallouk, T.E., and Fendler, J.H., Layer-by-Layer Assembly of Thin Film Zener Diodes from Conducting Polymers and Cdse Nanoparticles. J. Am. Chem. Soc., 1998. 120, 7848.

106. Yang, C.C., Y. Josefowicz, J., and Alexandru, L., Deposition of Ultrathin Films by a Withdrawal Method. Thin Solid Films, 1980. 74, 117.

107. Achermann, M., Petruska, M.A., Crooker, S.A., and Klimov, V.I., Picosecond Energy Transfer in Quantum Dot Langmuir-Blodgett Nanoassemblies. J. Phys. Chem. B, 2003. 107, 13782.

108. Shimomura, M. and Sawadaishi, T., Bottom-up Strategy of Materials Fabrication: A New Trend in Nanotechnology of Soft Materials. Curr. Opin. Colloid Interface Sci., 2001. 6, 11.

109. Fu, Y., Xu, H., Bai, S., Qiu, D., Sun, J., Wang, Z., and Zhang, X., Fabrication of a Stable Polyelectrolyte/Au Nanoparticles Multilayer Film. Macromol. Rapid Commun., 2002. 23, 256.

110. Gu, G., Garbuzov, D.Z., Burrows, P.E., Venkatesh, S., Forrest, S.R., and Thompson, M.E., High-External-Quantum-Efficiency Organic Light-Emitting Devices. Optics Letters, 1997. 22(6), 396-398.

111. Nakamura, S., Kitamura, K., Umeya, H., Jia, A., Kobayashi, M., Yoshikawa, A., Shimotomai, M., Kato, Y., and Takahashi, K., Bright Electroluminescence from Cds Quantum Dot Led Structures. Electronics Letters, 1998. 34(25), 2435-2436.

112. Coe, S., Woo, W.K., Bawendi, M., and Bulovic, V., Electroluminescence from Single Monolayers of Nanocrystals in Molecular Organic Devices. Nature, 2002. 420, 800 .

113. Sun, Q., Wang, Y.A., Li, L.S., Wang, D., Zhu, T., Xu, J., Yang, C., and Li, Y., Bright, Multicoloured Light-Emitting Diodes Based on Quantum Dots. Nature Photonics, 2007. 1(12), 717-722. 
114. Cho, K.-S., Lee, E.K., Joo, W.-J., Jang, E., Kim, T.-H., Lee, S.J., Kwon, S.-J., Han, J.Y., Kim, B.-K., and Choi, B.L., High-Performance Crosslinked Colloidal Quantum-Dot Light-Emitting Diodes. Nature Photonics, 2009. 3(6), 341-345.

115. Caruge, J., Halpert, J., Wood, V., Bulović, V., and Bawendi, M., Colloidal QuantumDot Light-Emitting Diodes with Metal-Oxide Charge Transport Layers. Nature Photonics, 2008. 2(4), 247-250.

116. Colvin, V.L., Schlamp, M.C., and Alivisatos, A.P., Light-Emitting Diodes Made from Cadmium Selenide Nanocrystals and a Semiconducting Polymer. Nature, 1994. $370,354$.

117. Steckel, J.S., Coe-Sullivan, S., Bulović, V., and Bawendi, M.G., 1.3 Mm to 1.55 Mm Tunable Electroluminescence from Pbse Quantum Dots Embedded within an Organic Device. Adv. Mater., 2003. 15, 1862.

118. O’Connor, É., O’Riordan, A., Doyle, H., Moynihan, S., Cuddihy, A., and Redmond, G., Near-Infrared Electroluminescent Devices Based on Colloidal Hgte Quantum Dot Arrays. Applied Physics Letters, 2005. 86(20), 201114.

119. Hyun, B.R., Choi, J.J., Seyler, K.L., Hanrath, T., and Wise, F.W., Heterojunction Pbs Nanocrystal Solar Cells with Oxide Charge-Transport Layers. ACS Nano, 2013. 7, 10938.

120. Caruge, J.-M., Halpert, J.E., Bulović, V., and Bawendi, M.G., Nio as an Inorganic Hole-Transporting Layer in Quantum-Dot Light-Emitting Devices. Nano letters, 2006. 6(12), 2991-2994.

121. Huang, L., Tu, C.C., and Lin, L.Y., Colloidal Quantum Dot Photodetectors Enhanced by Self-Assembled Plasmonic Nanoparticles. Appl. Phys. Lett., 2011. 98, 113110.

122. McDonald, S.A., Konstantatos, G., Zhang, S., Cyr, P.W., Klem, E.J.D., Levina, L., and Sargent, E.H., Solution-Processed Pbs Quantum Dot Infrared Photodetectors and Photovoltaics. Nat. Mater., 2005. 4, 138.

123. Oertel, D.C., Bawendi, M.G., Arango, A.C., and Bulović, V., Photodetectors Based on Treated Cdse Quantum-Dot Films. Appl. Phys. Lett., 2005. 87, 213505.

124. Chen, H.S., Wang, S.J.J., Lo, C.J., and Chi, J.Y., White-Light Emission from Organics-Capped Znse Quantum Dots and Application in White-Light-Emitting Diodes. Applied Physics Letters, 2005. 86(13), 131905.

125. Chen, H.-S., Hsu, C.-K., and Hong, H.-Y., Ingan-Cdse-Znse Quantum Dots White Leds. Ieee photonics technology letters, 2006. 18(1), 193-195.

126. Konstantatos, G., Howard, I., Fischer, A., Hoogland, S., Clifford, J., Klem, E., Levina, L., and Sargent, E.H., Ultrasensitive Solution-Cast Quantum Dot Photodetectors. Nature, 2006. 442, 180.

127. Keuleyan, S., Lhuillier, E., Brajuskovic, V., and Guyot-Sionnest, P., Mid-Infrared Hgte Colloidal Quantum Dot Photodetectors. Nat. Photonics, 2011. 5, 489.

128. Diedenhofen, S.L., Kufer, D., Lasanta, T., and Konstantatos, G., Integrated Colloidal Quantum Dot Photodetectors with Color-Tunable Plasmonic Nanofocusing Lenses. Light Sci. Appl., 2015. 4, e234.

129. Lee, J., Sundar, V.C., Heine, J.R., Bawendi, M.G., and Jensen, K.F., Full Color Emission from Ii-Vi Semiconductor Quantum Dot-Polymer Composites. Advanced Materials, 2000. 12(15), 1102-1105. 
130. Bailey, R.E., Smith, A.M., and Nie, S., Quantum Dots in Biology and Medicine. Physica E: Low-dimensional Systems and Nanostructures, 2004. 25(1), 1-12.

131. Bera, D., Qian, L., and Holloway, P.H., Semiconducting Quantum Dots for Bioimaging, in Drug Delivery Nanoparticles Formulation and Characterization. 2009, CRC Press. p. 349-366.

132. Smith, A.M., Duan, H.W., Mohs, A.M., and Nie, S.M., Bioconjugated Quantum Dots for in Vivo Molecular and Cellular Imaging. Adv. Drug Delivery Rev., 2008. 60, 1226.

133. Dahan, M., Levi, S., Luccardini, C., Rostaing, P., Riveau, B., and Triller, A., Diffusion Dynamics of Glycine Receptors Revealed by Single-Quantum Dot Tracking. Science, 2003. 302(5644), 442-445.

134. Sukhanova, A., Devy, J., Venteo, L., Kaplan, H., Artemyev, M., Oleinikov, V., Klinov, D., Pluot, M., Cohen, J.H., and Nabiev, I., Biocompatible Fluorescent Nanocrystals for Immunolabeling of Membrane Proteins and Cells. Analytical biochemistry, 2004. 324(1), 60-67.

135. Sapsford, K.E., Pons, T., Medintz, I.L., and Mattoussi, H., Biosensing with Luminescent Semiconductor Quantum Dots. Sensors, 2006. 6(8), 925-953.

136. Medintz, I.L., Uyeda, H.T., Goldman, E.R., and Mattoussi, H., Quantum Dot Bioconjugates for Imaging, Labelling and Sensing. Nature materials, 2005. 4(6), 435-446.

137. Hassan, M. and Klaunberg, B.A., Biomedical Applications of Fluorescence Imaging in Vivo. Comparative medicine, 2004. 54(6), 635-644.

138. Michalet, X., Pinaud, F., Bentolila, L., Tsay, J., Doose, S., Li, J., Sundaresan, G., Wu, A., Gambhir, S., and Weiss, S., Quantum Dots for Live Cells, in Vivo Imaging, and Diagnostics. science, 2005. 307(5709), 538-544.

139. Clapp, A.R., Medintz, I.L., Fisher, B.R., Anderson, G.P., and Mattoussi, H., Can Luminescent Quantum Dots Be Efficient Energy Acceptors with Organic Dye Donors? Journal of the American Chemical Society, 2005. 127(4), 1242-1250.

140. Clapp, A.R., Medintz, I.L., Mauro, J.M., Fisher, B.R., Bawendi, M.G., and Mattoussi, H., Fluorescence Resonance Energy Transfer between Quantum Dot Donors and Dye-Labeled Protein Acceptors. Journal of the American Chemical Society, 2004. 126(1), 301-310.

141. Tran, P., Goldman, E., Anderson, G., Mauro, J., and Mattoussi, H., Use of Luminescent Cdse-Zns Nanocrystal Bioconjugates in Quantum Dot-Based Nanosensors. 2002, DTIC Document.

142. Qian, X., Peng, X.-H., Ansari, D.O., Yin-Goen, Q., Chen, G.Z., Shin, D.M., Yang, L., Young, A.N., Wang, M.D., and Nie, S., In Vivo Tumor Targeting and Spectroscopic Detection with Surface-Enhanced Raman Nanoparticle Tags. Nat Biotech, 2008. 26(1), 83-90.

143. Reuter, C., Morgan, M., and Eckardt, A., Targeting Egf-Receptor-Signalling in Squamous Cell Carcinomas of the Head and Neck. British journal of cancer, 2007. 96(3), 408-416.

144. Herbst, R.S. and Shin, D.M., Monoclonal Antibodies to Target Epidermal Growth Factor Receptor-Positive Tumors. Cancer, 2002. 94(5), 1593-1611.

145. Tan, W.B. and Zhang, Y., Multifunctional Quantum-Dot-Based Magnetic Chitosan Nanobeads. Advanced Materials, 2005. 17(19), 2375-2380. 
146. Pierre, V.C., Botta, M., and Raymond, K.N., Dendrimeric Gadolinium Chelate with Fast Water Exchange and High Relaxivity at High Magnetic Field Strength. Journal of the American Chemical Society, 2005. 127(2), 504-505.

147. Rohrer, M., Bauer, H., Mintorovitch, J., Requardt, M., and Weinmann, H.-J., Comparison of Magnetic Properties of Mri Contrast Media Solutions at Different Magnetic Field Strengths. Investigative radiology, 2005. 40(11), 715-724.

148. Tóth, É., Bolskar, R.D., Borel, A., González, G., Helm, L., Merbach, A.E., Sitharaman, B., and Wilson, L.J., Water-Soluble Gadofullerenes: Toward HighRelaxivity, Ph-Responsive Mri Contrast Agents. Journal of the American Chemical Society, 2005. 127(2), 799-805.

149. Paschotta, R. and RP-Photonics. Ultrashort Pulses. Available from: https://www.rpphotonics.com/ultrashort pulses.html.

150. Chini, M., Zhao, K., and Chang, Z., The Generation, Characterization and Applications of Broadband Isolated Attosecond Pulses. Nat Photon, 2014. 8(3), 178186.

151. NobelPrize.Org. The Nobel Prize in Chemistry 1999. 27 May 2017; Available from: http://www.nobelprize.org/nobel prizes/chemistry/laureates/1999/.

152. Zewail, A.H., Femtochemistry. Past, Present, and Future. Pure and applied chemistry, 2000. 72(12), 2219-2231.

153. Hertz, H., Ueber Den Einfluss Des Ultravioletten Lichtes Auf Die Electrische Entladung (on an Effect of Ultra-Violet Light Upon the Electrical Discharge). Annalen der Physik., 1887. 267(8), 983-1000.

154. Einstein, A., Über Einen Die Erzeugung Und Verwandlung Des Lichtes Betreffenden Heuristischen Gesichtspunkt. Annalen der Physik, 1905. 17(6), 132-148.

155. Hüfner, S., Photoelectron Spectroscopy: Principles and Applications. 2003: Springer.

156. Fromhold, A.T., Quantum Mechanics for Applied Physics and Engineering. 1991: Courier Dover Publications.

157. Svelto, O., Principles of Lasers 5th Edition. 2010: Springer.

158. Silfvast, W.T., Laser Fundamentals 2nd Edition. 2008: Cambridge University Press.

159. Bertolotti, M., The History of the Laser. 2005: IOP Publishing Ltd.

160. LaserFest. Laser History. 2017; Available from: http://laserfest.org/lasers/history/index.cfm.

161. WorldofLasers. History of Lasers. 2015; Available from: http://www.worldoflasers.com/laserhistory.htm.

162. OregonStateUniversity. Laser Types and Classification. 2017; Available from: http://oregonstate.edu/ehs/laser/training/laser-types-and-classification.

163. Rullière, C. and SpringerLink (Online service), Femtosecond Laser Pulses Principles and Experiments, in Advanced texts in physics,. 2005, Springer Science+Business Media, Inc.,: New York, NY.

164. Moulton, P.F., Spectroscopic and Laser Characteristics of Ti:Al2o3. Journal of the Optical Society of America B, 1986. 3(1), 125-133.

165. Heisenberg, W., Über Den Anschaulichen Inhalt Der Quantentheoretischen Kinematik Und Mechanik. Zeitschrift für Physik, 1927. 43(3), 172-198. 
166. Niebauer, T.M., Faller, J.E., Godwin, H.M., Hall, J.L., and Barger, R.L., Frequency Stability Measurements on Polarization-Stabilized He-Ne Lasers. Applied Optics, 1988. 27(7), 1285-1289.

167. Paschotta, R. and RP-Photonics. Gaussian Pulses Calculator. Available from: https://www.rp-photonics.com/gaussian pulses.html.

168. Kerr, J., Xl. A New Relation between Electricity and Light: Dielectrified Media Birefringent. Philosophical Magazine Series 4, 1875. 50(332), 337-348.

169. Kerr, J., Liv. A New Relation between Electricity and Light: Dielectrified Media Birefringent (Second Paper). Philosophical Magazine Series 4, 1875. 50(333), 446458.

170. Bloembergen, N., Nonlinear Optics; a Lecture Note and Reprint Volume. Frontiers in Physics. 1965, New York,: W. A. Benjamin. xiii, 222 p.

171. Elsaesser, T., Fujimoto, J.G., Wiersma, D.A., and Zinth, W., Ultrafast Phenomena Xi 1998, Springer: Berlin, Heidelberg.

172. French, P.M.W., The Generation of Ultrashort Laser Pulses. Reports on Progress in Physics, 1995. 58(2), 169.

173. Maine, P., Strickland, D., Bado, P., Pessot, M., and Mourou, G., Generation of Ultrahigh Peak Power Pulses by Chirped Pulse Amplification. IEEE Journal of Quantum Electronics, 1988. 24(2), 398-403.

174. Clark-MXR, Cpa-Series User Manual Version 2.5. 2010, Copyright reserved by Clark-MXR, Inc.: Dexter, Michigan.

175. Paschotta, R. and RP-Photonics. Pockels Cell. Available from: https://www.rpphotonics.com/pockels cells.html? s=ak.

176. Paschotta, R. and RP-Photonics. Pockels Effect. Available from: https://www.rpphotonics.com/pockels effect.html.

177. Modoran, G.C., Interaction of Ultrashort Pulses with Transparent Materials. 2005, Ohio State University.

178. Eckardt, R. and Reintjes, J., Phase Matching Limitations of High Efficiency Second Harmonic Generation. IEEE Journal of Quantum Electronics, 1984. 20(10), 11781187.

179. Alfano, R.R., The Supercontinuum Laser Source. 1989, New York: Springer-Verlag. xiv, $458 \mathrm{p}$.

180. Alfano, R.R. and Shapiro, S.L., Emission in the Region 4000 to 7000 a Via FourPhoton Coupling in Glass. Physical Review Letters, 1970. 24(11), 584-587.

181. R.L. Fork, C.V. Shank, and R.T. Yen, Amplification of 70-Fs Optical Pulses to Gigawatt Powers. Appl. Phys. Lett. , 1983. 41, 223-225.

182. Sanders, S.T., Wavelength-Agile Fiber Laser Using Group-Velocity Dispersion of Pulsed Super-Continua and Application to Broadband Absorption Spectroscopy. Applied Physics B, 2002. 75(6), 799-802.

183. Morioka, T., Mori, K., and Saruwatari, M., More Than 100-Wavelength-Channel Picosecond Optical Pulse Generation from Single Laser Source Using Supercontinuum in Optical Fibres. Institution of Engineering and Technology, 1993. 29(10), 862-864.

184. Lanigan, P.M., Dunsby, C., McGinty, J., Elson, D.S., Requejo-Isidro, J., Munro, I., Galletly, N., Treanor, B., Önfelt, B., McCann, F., Davis, D.M., Neil, M.A., and French, P.M. An Electronically Tunable Ultrafast Laser Source Applied to 
Fluorescence Imaging and Fluorescence Lifetime Imaging Microscopy. in Conference on Lasers and Electro-Optics/Quantum Electronics and Laser Science and Photonic Applications Systems Technologies. 2005. Baltimore, Maryland: Optical Society of America.

185. M. Ere-Tassou, C. Przygodzki, E. Fertein, and H. Delbarre, Femtosecond Laser Source for Real-Time Atmospheric Gas Sensing in the Uv-Visible. Opt. Commun., 2003(220), 215-221.

186. Zheltikov, A., Supercontinuum Generation. Appl. Phys. B, 2003(77), 143-147.

187. R. Trebino, K. W. DeLong, D. N. Fittinghoff, J. N. Sweetser, M. A. Krumbügel, and D. J. Kane, Measuring Ultrashort Laser Pulses in the Time-Frequency Domain Using Frequency-Resolved Optical Gating. Review of Scientific Instruments, 1997. 68(9).

188. O'Shea, P., Kimmel, M., Gu, X., and Trebino, R., Highly Simplified Device for Ultrashort-Pulse Measurement. Optics Letters, 2001. 26(12), 932-934.

189. Iaconis, C. and Walmsley, I.A., Self-Referencing Spectral Interferometry for Measuring Ultrashort Optical Pulses. IEEE Journal of Quantum Electronics, 1999. 35(4), 501-509.

190. Lippert, H., Ritze, H.H., Hertel, I.V., and Radloff, W., Femtosecond Time-Resolved Hydrogen-Atom Elimination from Photoexcited Pyrrole Molecules. ChemPhysChem, 2004. 5(9), 1423-1427.

191. Schanz, H.R., Femtoseond Broadband-Fluoresene up Onversion. 2002, HumboldtUniversität zu Berlin.

192. W_A_Hammond_Drierite, L. Regeneration of Indicating Drierite Desiccants. Available from: https://secure.drierite.com/catalog3/page19b.cfm.

193. Clark-MXR, Nopa User Manual, Version 1.5. 2010, Copyright reserved by ClarkMXR, Inc.: Dexter, Michigan.

194. Liu, J., Jamhawi, A.M., and Xie, Y. Lock-in Amplifier. 2015; Available from: https://sites.google.com/site/uofllasergroupwiki/lock-in-amplifier.

195. Clark-MXR, Tapps User Manual, Version 1.5. 2010, Copyright reserved by ClarkMXR, Inc.: Dexter, Michigan.

196. Liu, J., Jamhawi, A.M., and Xie, Y. Time Zeros in the Ultrafast System. 2015; Available from: https://sites.google.com/site/uofllasergroupwiki/time-zeros.

197. Bang, J.H. and Kamat, P.V., Quantum Dot Sensitized Solar Cells. A Tale of Two Semiconductor Nanocrystals: Cdse and Cdte. ACS Nano, 2009. 3, 1467.

198. Gur, I., Fromer, N.A., Geier, M.L., and Alivisatos, A.P., Air-Stable All-Inorganic Nanocrystal Solar Cells Processed from Solution. Science, 2005. 310, 462.

199. Sargent, E.H., Infrared Photovoltaics Made by Solution Processing. Nat. Photonics, 2009. $3,325$.

200. Tang, J., Kemp, K.W., Hoogland, S., Jeong, K.S., Liu, H., Levina, L., Furukawa, M., Wang, X., Debnath, R., and Cha, D., Colloidal-Quantum-Dot Photovoltaics Using Atomic-Ligand Passivation. Nat. Mater., 2011. 10, 765.

201. Tvrdy, K., Frantsuzov, P.A., and Kamat, P.V., Photoinduced Electron Transfer from Semiconductor Quantum Dots to Metal Oxide Nanoparticles. Proc. Natl. Acad. Sci. U. S. A., 2011. 108, 29.

202. Yu, D., Wang, C., and Guyot-Sionnest, P., N-Type Conducting Cdse Nanocrystal Solids. Science, 2003. 300, 1277. 
203. Wang, C., Shim, M., and Guyot-Sionnest, P., Electrochromic Nanocrystal Quantum Dots. Science, 2001. 291, 2390.

204. Kovalenko, M.V., Scheele, M., and Talapin, D.V., Colloidal Nanocrystals with Molecular Metal Chalcogenide Surface Ligands. Science, 2009. 324, 1417.

205. Lee, J.S., Kovalenko, M.V., Huang, J., Chung, D.S., and Talapin, D.V., Band-Like Transport, High Electron Mobility and High Photoconductivity in All-Inorganic Nanocrystal Arrays. Nat. Nanotechnol., 2011. 6, 348.

206. Talapin, D.V., Lee, J.S., Kovalenko, M.V., and Shevchenko, E.V., Prospects of Colloidal Nanocrystals for Electronic and Optoelectronic Applications. Chem. Rev., 2009. $110,389$.

207. Talapin, D.V. and Murray, C.B., Pbse Nanocrystal Solids for N-and P-Channel Thin Film Field-Effect Transistors. Science, 2005. 310, 86.

208. Huang, J., Stockwell, D., Huang, Z., Mohler, D.L., and Lian, T., Photoinduced Ultrafast Electron Transfer from Cdse Quantum Dots to Re-Bipyridyl Complexes. J. Am. Chem. Soc., 2008. 130, 5632.

209. Sykora, M., Petruska, M.A., Alstrum-Acevedo, J., Bezel, I., Meyer, T.J., and Klimov, V.I., Photoinduced Charge Transfer between Cdse Nanocrystal Quantum Dots and Ru-Polypyridine Complexes. J. Am. Chem. Soc., 2006. 128, 9984.

210. Yang, Y., Rodríguez-Córdoba, W., and Lian, T., Ultrafast Charge Separation and Recombination Dynamics in Lead Sulfide Quantum Dot-Methylene Blue Complexes Probed by Electron and Hole Intraband Transitions. J. Am. Chem. Soc., 2011. 133, 9246.

211. Anderson, N.A. and Lian, T., Ultrafast Electron Transfer at the MoleculeSemiconductor Nanoparticle Interface. Annu. Rev. Phys. Chem., 2005. 56, 491.

212. Kambhampati, P., Unraveling the Structure and Dynamics of Excitons in Semiconductor Quantum Dots. Acc. Chem. Res., 2011. 44, 1.

213. Kongkanand, A., Tvrdy, K., Takechi, K., Kuno, M., and Kamat, P.V., Quantum Dot Solar Cells. Tuning Photoresponse through Size and Shape Control of Cdse-Tio2 Architecture. J. Am. Chem. Soc., 2008. 130, 4007.

214. Meier, A., Selmarten, D.C., Siemoneit, K., Smith, B.B., and Nozik, A.J., Fast Electron Transfer across Semiconductor-Molecule Interfaces: Gaas/Co(Cp)2+/0. J. Phys. Chem. B, 1999. 103, 2122.

215. Norris, D.J., Sacra, A., Murray, C.B., and Bawendi, M.G., Measurement of the Size Dependent Hole Spectrum in Cdse Quantum Dots. Phys. Rev. Lett., 1994. 72, 2612.

216. Nozik, A.J., Spectroscopy and Hot Electron Relaxation Dynamics in Semiconductor Quantum Wells and Quantum Dots. Annu. Rev. Phys. Chem., 2001. 52, 193.

217. Rinehart, J.D., Schimpf, A.M., Weaver, A.L., Cohn, A.W., and Gamelin, D.R., Photochemical Electronic Doping of Colloidal Cdse Nanocrystals. J. Am. Chem. Soc., 2013. 135, 18782.

218. Robel, I., Subramanian, V., Kuno, M., and Kamat, P.V., Quantum Dot Solar Cells. Harvesting Light Energy with Cdse Nanocrystals Molecularly Linked to Mesoscopic Tio2 Films. J. Am. Chem. Soc., 2006. 128, 2385.

219. Xu, S., Mikhailovsky, A.A., Hollingsworth, J.A., and Klimov, V.I., Hole Intraband Relaxation in Strongly Confined Quantum Dots: Revisiting the "Phonon Bottleneck" Problem. Phys. Rev. B, 2002. 65, 045319. 
220. Bowers, M.J., McBride, J.R., Garrett, M.D., Sammons, J.A., Dukes, A.D., Schreuder, M.A., Watt, T.L., Lupini, A.R., Pennycook, S.J., and Rosenthal, S.J., Structure and Ultrafast Dynamics of White-Light-Emitting Cdse Nanocrystals. J. Am. Chem. Soc., 2009. 131, 5730.

221. Prezhdo, O.V., Photoinduced Dynamics in Semiconductor Quantum Dots: Insights from Time-Domain Ab Initio Studies. Acc. Chem. Res., 2009. 42, 2005.

222. Hedrick, M.M., Mayo, M.L., Badaeva, E., and Kilina, S., First-Principles Studies of the Ground- and Excited-State Properties of Quantum Dots Functionalized by Ru(Ii)-Polybipyridine. J. Phys. Chem. C, 2013. 117, 18216.

223. Long, R., English, N.J., and Prezhdo, O.V., Defects Are Needed for Fast PhotoInduced Electron Transfer from a Nanocrystal to a Molecule: Time-Domain Ab Initio Analysis. J. Am. Chem. Soc., 2013. 135, 18892.

224. Steigerwald, M.L. and Brus, L.E., Semiconductor Crystallites: A Class of Large Molecules. Acc. Chem. Res., 1990. 23, 183.

225. Kasuya, A., Sivamohan, R., Barnakov, Y.A., Dmitruk, I.M., Nirasawa, T., Romanyuk, V.R., Kumar, V., Mamykin, S.V., Tohji, K., Jeyadevan, B., Shinoda, K., Kudo, T., Terasaki, O., Liu, Z., Belosludov, R.V., Sundararajan, V., and Kawazoe, Y., Ultra-Stable Nanoparticles of Cdse Revealed from Mass Spectrometry. Nat. Mater., 2004. 3, 99.

226. Teunis, M.B., Dolai, S., and Sardar, R., Effects of Surface-Passivating Ligands and Ultrasmall Cdse Nanocrystal Size on the Delocalization of Exciton Confinement. Langmuir, 2014. 30, 7851.

227. Seker, F., Meeker, K., Kuech, T.F., and Ellis, A.B., Surface Chemistry of Prototypical Bulk Ii-Vi and Iii-V Semiconductors and Implications for Chemical Sensing. Chem. Rev., 2000. 100, 2505.

228. Hoffmann, R., A Chemical and Theoretical Way to Look at Bonding on Surfaces. Rev. Mod. Phys., 1988. 60, 601.

229. Wessels, J.M., Nothofer, H.G., Ford, W.E., von Wrochem, F., Scholz, F., Vossmeyer, T., Schroedter, A., Weller, H., and Yasuda, A., Optical and Electrical Properties of Three-Dimensional Interlinked Gold Nanoparticle Assemblies. J. Am. Chem. Soc., 2004. 126, 3349.

230. Dolai, S., Nimmala, P.R., Mandal, M., Muhoberac, B.B., Dria, K., Dass, A., and Sardar, R., Isolation of Bright Blue Light-Emitting Cdse Nanocrystals with $6.5 \mathrm{Kda}$ Core in Gram Scale: High Photoluminescence Efficiency Controlled by Surface Ligand Chemistry. Chemistry of Materials, 2014. 26(2), 1278-1285.

231. Pandit, B., Luitel, T., Cummins, D.R., Thapa, A.K., Druffel, T., Zamborini, F., and Liu, J., Spectroscopic Investigation of Photoinduced Charge-Transfer Processes in Fto/Tio2/N719 Photoanodes with and without Covalent Attachment through SilaneBased Linkers. J. Phys. Chem. A, 2013. 117, 13513.

232. Norris, D.J. and Bawendi, M.G., Measurement and Assignment of the SizeDependent Optical Spectrum in Cdse Quantum Dots. Phys. Rev. B, 1996. 53, 16338.

233. Ekimov, A.I., Hache, F., Schanne-Klein, M.C., Ricard, D., Flytzanis, C., Kudryavtsev, I.A., Yazeva, T.V., Rodina, A.V., and Efros, A.L., Absorption and Intensity-Dependent Photoluminescence Measurements on Cdse Quantum Dots: Assignment of the First Electronic Transitions. J. Opt. Soc. Am. B, 1993. 10, 100. 
234. Sewall, S.L., Cooney, R.R., Anderson, K.E.H., Dias, E.A., Sagar, D.M., and Kambhampati, P., State-Resolved Studies of Biexcitons and Surface Trapping Dynamics in Semiconductor Quantum Dots. J. Chem. Phys., 2008. 129, 084701.

235. Malicki, M., Knowles, K.E., and Weiss, E.A., Gating of Hole Transfer from Photoexcited Pbs Quantum Dots to Aminoferrocene by the Ligand Shell of the Dots. Chem. Commun., 2013. 49, 4400.

236. Rawalekar, S., Kaniyankandy, S., Verma, S., and Ghosh, H.N., Ultrafast Charge Carrier Relaxation and Charge Transfer Dynamics of Cdte/Cds Core-Shell Quantum Dots as Studied by Femtosecond Transient Absorption Spectroscopy. J. Phys. Chem. C, 2010. 114, 1460.

237. Wu, F., Zhang, J.Z., Kho, R., and Mehra, R.K., Radiative and Nonradiative Lifetimes of Band Edge States and Deep Trap States of Cds Nanoparticles Determined by Time-Correlated Single Photon Counting. Chem. Phys. Lett., 2000. 330, 237.

238. Crooker, S.A., Barrick, T., Hollingsworth, J.A., and Klimov, V.I., Multiple Temperature Regimes of Radiative Decay in Cdse Nanocrystal Quantum Dots: Intrinsic Limits to the Dark-Exciton Lifetime. Appl. Phys. Lett., 2003. 82, 2793.

239. Klimov, V., Hunsche, S., and Kurz, H., Biexciton Effects in Femtosecond Nonlinear Transmission of Semiconductor Quantum Dots. Phys. Rev. B, 1994. 50, 8110.

240. Klimov, V.I., Optical Nonlinearities and Ultrafast Carrier Dynamics in Semiconductor Nanocrystals. J. Phys. Chem. B, 2000. 104, 6112.

241. Kucur, E., Riegler, J., Urban, G.A., and Nann, T., Determination of Quantum Confinement in Cdse Nanocrystals by Cyclic Voltammetry. J. Chem. Phys., 2003. $119,2333$.

242. Querner, C., Reiss, P., Sadki, S., Zagorska, M., and Pron, A., Size and Ligand Effects on the Electrochemical and Spectroelectrochemical Responses of Cdse Nanocrystals. Phys. Chem. Chem. Phys., 2005. 7, 3204.

243. Frederick, M.T., Amin, V.A., Cass, L.C., and Weiss, E.A., A Molecule to Detect and Perturb the Confinement of Charge Carriers in Quantum Dots. Nano Lett., 2011. $11,5455$.

244. Grancini, G., Polli, D., Fazzi, D., Cabanillas-Gonzalez, J., Cerullo, G., and Lanzani, G., Transient Absorption Imaging of P3ht:Pcbm Photovoltaic Blend: Evidence for Interfacial Charge Transfer State. J. Phys. Chem. Lett., 2011. 2, 1099.

245. Jailaubekov, A.E., Willard, A.P., Tritsch, J.R., Chan, W.L., Sai, N., Gearba, R., Kaake, L.G., Williams, K.J., Leung, K., Rossky, P.J., and Zhu, X.Y., Hot ChargeTransfer Excitons Set the Time Limit for Charge Separation at Donor/Acceptor Interfaces in Organic Photovoltaics. Nat. Mater., 2013. 12, 66.

246. Ross, R.T. and Nozik, A.J., Efficiency of Hot-Carrier Solar Energy Converters. J. Appl. Phys., 1982. 53, 3813.

247. Pandey, A. and Guyot-Sionnest, P., Hot Electron Extraction from Colloidal Quantum Dots. J. Phys. Chem. Lett., 2010. 1, 45.

248. Tisdale, W.A., Williams, K.J., Timp, B.A., Norris, D.J., Aydil, E.S., and Zhu, X.Y., Hot-Electron Transfer from Semiconductor Nanocrystals. Science, 2010. 328, 1543.

249. Kambhampati, P., Hot Exciton Relaxation Dynamics in Semiconductor Quantum Dots: Radiationless Transitions on the Nanoscale. J. Phys. Chem. C, 2011. 115, 22089. 
250. Del Ben, M., Havenith, R.W.A., Broer, R., and Stener, M., Density Functional Study on the Morphology and Photoabsorption of Cdse Nanoclusters. J. Phys. Chem. C, 2011. 115, 16782.

251. Brus, L., Model for Carrier Dynamics and Photoluminescence Quenching in Wet and Dry Porous Silicon Thin Films. Phys. Rev. B, 1996. 53, 4649.

252. Sambur, J.B., Novet, T., and Parkinson, B.A., Multiple Exciton Collection in a Sensitized Photovoltaic System. Science, 2010. 330, 63.

253. Moreels, I., Lambert, K., De Muynck, D., Vanhaecke, F., Poelman, D., Martins, J.C., Allan, G., and Hens, Z., Composition and Size-Dependent Extinction Coefficient of Colloidal Pbse Quantum Dots. Chem. Mater., 2007. 19, 6101.

254. Alivisatos, A.P., Perspectives on the Physical Chemistry of Semiconductor Nanocrystals. J. Phys. Chem., 1996. 100, 13226.

255. Peng, Z.A. and Peng, X., Formation of High-Quality Cdte, Cdse, and Cds Nanocrystals Using Cdo as Precursor. J. Am. Chem. Soc., 2001. 123, 183.

256. Vossmeyer, T., Katsikas, L., Giersig, M., Popovic, I.G., Diesner, K., Chemseddine, A., Eychmuller, A., and Weller, H., Cds Nanoclusters - Synthesis, Characterzation, Size-Dependent Oscillator Strength, Temperature Shift of the Excitonic-Transition Energy, and Reversible Absorbency Shift. J. Phys. Chem., 1994. 98, 7665.

257. Ruhle, S., Shalom, M., and Zaban, A., Quantum-Dot-Sensitized Solar Cells. ChemPhysChem, 2010. 11, 2290.

258. Kamat, P.V., Quantum Dot Solar Cells. The Next Big Thing in Photovoltaics. J. Phys. Chem. Lett., 2013. 4, 908.

259. Knowles, K.E., Frederick, M.T., Tice, D.B., Morris-Cohen, A.J., and Weiss, E.A., Colloidal Quantum Dots: Think Outside the (Particle-in-a-)Box. J. Phys. Chem. Lett., 2012. 3, 18.

260. Singh, V.A., Ranjan, V., and Kapoor, V., Semiconductor Quantum Dots: Theory and Phenomenology. Bull. Mat. Sci., 1999. 22, 563.

261. Yoffe, A.D., Semiconductor Quantum Dots and Related Systems: Electronic, Optical, Luminescence and Related Properties of Low Dimensional Systems. Adv. Phys., 2001. 50, 1.

262. Hines, D.A. and Kamat, P.V., Recent Advances in Quantum Dot Surface Chemistry. ACS Applied Materials \& Interfaces, 2014. 6(5), 3041-3057.

263. Dabbousi, B.O., Rodriguez-Viejo, J., Mikulec, F.V., Heine, J.R., Mattoussi, H., Ober, R., Jensen, K.F., and Bawendi, M.G., (Cdse)Zns Core-Shell Quantum Dots: Synthesis and Characterization of a Size Series of Highly Luminescent Nanocrystallites. J. Phys. Chem. B, 1997. 101, 9463.

264. Chuang, C.H., Lo, S.S., Scholes, G.D., and Burda, C., Charge Separation and Recombination in Cdte/Cdse Core/Shell Nanocrystals as a Function of Shell Coverage: Probing the Onset of the Quasi Type-Ii Regime. J. Phys. Chem. Lett., 2010. 1, 2530.

265. Wuister, S.F., de Mello Donega, C., and Meijerink, A., Influence of Thiol Capping on the Exciton Luminescence and Decay Kinetics of Cdte and Cdse Quantum Dots. J. Phys. Chem. B, 2004. 108, 17393.

266. Munro, A.M., Jen-La Plante, I., Ng, M.S., and Ginger, D.S., Quantitative Study of the Effects of Surface Ligand Concentration on Cdse Nanocrystal Photoluminescence. J. Phys. Chem. C., 2007. 111, 6220. 
267. Xu, J., Teng, Y., and Teng, F., Effect of Surface Defect States on Valence Band and Charge Separation and Transfer Efficiency. Scientific Reports, 2016. 6, 32457.

268. Amelia, M., Impellizzeri, S., Monaco, S., Yildiz, I., Silvi, S., Raymo, F.M., and Credi, A., Structural and Size Effects on the Spectroscopic and Redox Properties of Cdse Nanocrystals in Solution: The Role of Defect States. ChemPhysChem, 2011. $12,2280$.

269. Chaban, V.V., Prezhdo, V.V., and Prezhdo, O.V., Covalent Linking Greatly Enhances Photoinduced Electron Transfer in Fullerene-Quantum Dot Nanocomposites: Time-Domain Ab Initio Study. J. Phys. Chem. Lett., 2012. 4, 1.

270. Tan, Y., Jin, S., and Hamers, R.J., Influence of Hole-Sequestering Ligands on the Photostability of Cdse Quantum Dots. J. Phys. Chem. C, 2012. 117, 313.

271. Dibbell, R.S., Youker, D.G., and Watson, D.F., Excited-State Electron Transfer from Cds Quantum Dots to Tio2 Nanoparticles Via Molecular Linkers with Phenylene Bridges. J. Phys. Chem. C, 2009. 113, 18643.

272. Donakowski, M.D., Godbe, J.M., Sknepnek, R., Knowles, K.E., Olvera de la Cruz, M., and Weiss, E.A., A Quantitative Description of the Binding Equilibria of ParaSubstituted Aniline Ligands and Cdse Quantum Dots. J. Phys. Chem. C., 2010. 114, 22526.

273. Azpiroz, J.M. and De Angelis, F., Ligand Induced Spectral Changes in Cdse Quantum Dots. ACS Applied Materials \& Interfaces, 2015. 7(35), 19736-19745.

274. Zotti, G., Vercelli, B., Berlin, A., and Virgili, T., Multilayers of Cdse Nanocrystals and Bis(Dithiocarbamate) Linkers Displaying Record Photoconduction. The Journal of Physical Chemistry C, 2012. 116(49), 25689-25693.

275. Hansch, C., Leo, A., and Taft, R.W., A Survey of Hammett Substituent Constants and Resonance and Field Parameters. Chemical Reviews, 1991. 91(2), 165-195.

276. Lawrence, K.N., Dutta, P., Nagaraju, M., Teunis, M.B., Muhoberac, B.B., and Sardar, R., Dual Role of Electron-Accepting Metal-Carboxylate Ligands: Reversible Expansion of Exciton Delocalization and Passivation of Nonradiative Trap-States in Molecule-Like Cdse Nanocrystals. Journal of the American Chemical Society, 2016. 138(39), 12813-12825.

277. Kamat, P.V., Boosting the Efficiency of Quantum Dot Sensitized Solar Cells through Modulation of Interfacial Charge Transfer. Acc. Chem. Res., 2012. 45, 1906.

278. Christians, J.A. and Kamat, P.V., Trap and Transfer. Two-Step Hole Injection across the Sb2s3/Cuscn Interface in Solid-State Solar Cells. ACS Nano, 2013. 7, 7967.

279. Tan, Y., Jin, S., and Hamers, R.J., Photostability of Cdse Quantum Dots Functionalized with Aromatic Dithiocarbamate Ligands. ACS Applied Materials \& Interfaces, 2013. 5(24), 12975-12983.

280. Jin, S., Harris, R.D., Lau, B., Aruda, K.O., Amin, V.A., and Weiss, E.A., Enhanced Rate of Radiative Decay in Cdse Quantum Dots Upon Adsorption of an ExcitonDelocalizing Ligand. Nano Letters, 2014. 14(9), 5323-5328.

281. Harris, R.D., Amin, V.A., Lau, B., and Weiss, E.A., Role of Interligand Coupling in Determining the Interfacial Electronic Structure of Colloidal Cds Quantum Dots. ACS Nano, 2016. 10(1), 1395-1403.

282. McMurry, J.E., Organic Chemistry; Second Edition. 1987, United States: Wadsworth Publishing Co Inc. 
283. Lian, S., Weinberg, D.J., Harris, R.D., Kodaimati, M.S., and Weiss, E.A., Subpicosecond Photoinduced Hole Transfer from a Cds Quantum Dot to a Molecular Acceptor Bound through an Exciton-Delocalizing Ligand. ACS Nano, 2016. 10(6), 6372-6382.

284. Xiong, W., Hickstein, D.D., Schnitzenbaumer, K.J., Ellis, J.L., Palm, B.B., Keister, K.E., Ding, C., Miaja-Avila, L., Dukovic, G., Jimenez, J.L., Murnane, M.M., and Kapteyn, H.C., Photoelectron Spectroscopy of Cdse Nanocrystals in the Gas Phase: A Direct Measure of the Evanescent Electron Wave Function of Quantum Dots. Nano Letters, 2013. 13(6), 2924-2930. 


\section{APPENDIX I COPYRIGHT PERMISSION}

Figure 1.4. Solar Radiation Spectra.

Figure was prepared by Robert A. Rohde for the Global Warming Art project.. Permission for reprint is granted under the terms of GNU Free Documentation [22].

Figure 1.11. Schematic Illustrations of a Tandem QDSC.

Reprinted with permission from Reference [23]:

Santra, Pralay K.; Kamat, Prashant V. Tandem-Layered Quantum Dot Solar Cells: Tuning the Photovoltaic Response with Luminescent Ternary Cadmium Chalcogenides. J. Am. Chem. Soc., 2013, 135 (2), p877

Copyright 2013, American Chemistry Society.

Figure 1.7. Quantum Number-Denoted Energy Levels and Optical Interband Transitions. Figure adapted with permission from reference [27]:

Klimov, Victor I. Spectral and Dynamical Properties of Multilexcitons in Semiconductor Nanocrystals. Annu. Rev. Phys. Chem., 2007. 58, p635.

Copyright 2007, Annual Reviews. 
Figure 1.10. Wave function and Energy Level Alignments of PDTC - CdSe QDs.

Figure adapted with permission from reference [56]:

Frederick, Matthew T.; Amin, Victor A.; Swenson, Nathaniel K.; Ho, Andrew Y.; and Weiss, Emily A. Control of Exciton Confinement in Quantum Dot-Organic Complexes through Energetic Alignment of Interfacial Orbitals. Nano Letters, 2012. 13, p287.

Copyright 2012, American Chemistry Society.

Figure 1.11. Hot Injection Method to Synthesize QDs.

Reprinted with permission from Reference [70]

Carey, Graham H.; Abdelhady, Ahmed L.; Ning, Zhijun; Thon, Susanna M.; Bakr, Osman M.; and Sargent, Edward H. Colloidal Quantum Dot Solar Cells. Chem. Rev., 2015, 115 (23), p12732.

Copyright 2015, American Chemistry Society.

Figure 2.10. Frequency-resolved Optical Gating (FROG).

Reprinted with permission from Reference [187]:

Trebino, Rick; DeLong, Kenneth W.; Fittinghoff, David N.; Sweetser, John N.; Krumbügel, Marco A.; Richman, Bruce A. Measuring ultrashort laser pulses in the time-frequency domain using frequency-resolved optical gating. Review of Scientific Instruments, 1997. 68(9), p3277.

Copyright 1997, AIP Publishing. 
The Entire Chapter 3, PDTC Ligand Passivated CdSe SCNCs.

Reprinted with permission from Reference [31]:

Xie, Yizhou; Teunis, Meghan B.; Pandit, Bill; Sardar, Rajesh. Molecule-Like Cdse Nanoclusters Passivated with Strongly Interacting Ligands: Energy Level Alignment and Photoinduced Ultrafast Charge Transfer Processes. The Journal of Physical Chemistry C. 2015. 119(5), p2813.

Copyright 2015, American Chemistry Society.

Figure 4.1. HOMO Energy vs. the Hammett Coefficient.

Figure adapted with permission from Reference [56]:

Frederick, Matthew T.; Amin, Victor A.; Swenson, Nathaniel K.; Ho, Andrew Y.; and Weiss, Emily A. Control of Exciton Confinement in Quantum Dot-Organic Complexes through Energetic Alignment of Interfacial Orbitals. Nano Letters, 2012. 13, p287.

Copyright 2012, American Chemistry Society.

Procedures of X-PDTC Synthesis and Ligand Exchange Reactions in Chapter 4. Adapted with permission from Reference [226]:

Teunis, Meghan B.; Dolai, Sukanta; Bill; Sardar, Rajesh. Effects of Surface-Passivating Ligands and Ultrasmall Cdse Nanocrystal Size on the Delocalization of Exciton Confinement. Langmuir 2014. 30, p7851.

Copyright 2014, American Chemistry Society. 


\section{APPENDIX II MATHCAD GLOBAL FITTING}

\section{MathCAD Software Coding:}

$$
\mathrm{Y}_{\mathrm{IRF}}:=1 \quad \mathrm{t}_{\mathrm{zero}}:=0.22
$$

Area 1: Wave/time domain fuctions and $\triangle O D$ equation:

洒

The ultrafast transient absorption data is fit to the two-variable four-component function, convoluted by instrument response function (IRF):

$$
\begin{aligned}
& \mathrm{A}_{1}\left(\mathrm{Y}_{1}, \sigma, \lambda_{1}, \lambda\right):=\frac{\mathrm{Y}_{1}}{\sigma \sqrt{2 \pi}} \cdot \mathrm{e}^{\frac{-1}{2}} \cdot\left(\frac{\lambda-\lambda_{1}}{\sigma}\right)^{2} \quad \mathrm{~A}_{2}\left(\mathrm{Y}_{2}, \sigma, \lambda_{2}, \lambda\right):=\frac{\mathrm{Y}_{2}}{\sigma \sqrt{2 \pi}} \cdot \mathrm{e}^{\frac{-1}{2}} \cdot\left(\frac{\lambda-\lambda_{2}}{\sigma}\right)^{2} \\
& \mathrm{~A}_{3}\left(\mathrm{Y}_{3}, \sigma, \lambda_{3}, \lambda\right):=\frac{\mathrm{Y}_{3}}{\sigma \sqrt{2 \pi}} \cdot \mathrm{e}^{\frac{-1}{2}} \cdot\left(\frac{\lambda-\lambda_{3}}{\sigma}\right)^{2} \quad \mathrm{~A}_{4}\left(\mathrm{Y}_{4}, \sigma, \lambda_{4}, \lambda\right):=\frac{\mathrm{Y}_{4}}{\sigma \sqrt{2 \pi}} \cdot \mathrm{e}^{\frac{-1}{2}} \cdot\left(\frac{\lambda-\lambda_{4}}{\sigma}\right)^{2} \\
& \operatorname{IRF}\left(\sigma_{\mathrm{IRF}}, \mathrm{t}\right):=\frac{\mathrm{Y}_{\mathrm{IRF}}}{\sigma_{\mathrm{IRF}} \sqrt{2 \pi}} \cdot \mathrm{e}^{\frac{-1}{2} \cdot\left(\frac{\mathrm{t}}{\sigma_{\mathrm{IRF}}}\right)^{2}} \\
& I_{1}\left(\tau_{1 a}, \tau_{1 b}, \kappa, t\right):=\text { if }\left(t \leq t_{\text {zero }}, 0, \frac{\kappa}{\kappa+1} e^{\frac{-t}{\tau_{1 a}}}+\frac{1}{\kappa+1} \cdot e^{\frac{-t}{\tau} 1 b}\right) \\
& \mathrm{I}_{2}\left(\tau_{1 \mathrm{a}}, \tau_{1 \mathrm{~b}}, \tau_{2}, \kappa, \eta, \mathrm{t}\right):=\text { if }\left(\mathrm{t} \leq \mathrm{t}_{\text {zero }}, 0, \frac{\eta}{\eta+\kappa+1} \mathrm{e}^{\frac{-\mathrm{t}}{\tau_{2}}}+\frac{\kappa}{\eta+\kappa+1} \mathrm{e}^{\frac{-\mathrm{t}}{\tau_{1 a}}}+\frac{1}{\eta+\kappa+1} \cdot \mathrm{e}^{\frac{-\mathrm{t}}{\tau_{1 b}}}\right) \\
& I_{4}\left(k_{4 a}, k_{4 b}, I_{4 c}, t\right):=\text { if }\left[t<t_{z e r o}, 0, \frac{k_{4 a}}{k_{4 b}-k_{4 a}} \cdot\left(e^{-k_{4 a} \cdot t}-e^{\left.-k_{4 b} \cdot t\right)}+I_{4 c}\right]\right. \\
& \Delta O D\left(\mathrm{Y}_{1}, \mathrm{Y}_{2}, \mathrm{Y}_{3}, \mathrm{Y}_{4}, \sigma, \tau_{1 \mathrm{a}}, \tau_{1 \mathrm{~b}}, \tau_{2}, \mathrm{k}_{4 \mathrm{a}}, \mathrm{k}_{4 \mathrm{~b}}, \lambda_{1}, \lambda_{2}, \lambda_{3}, \lambda_{4}, \kappa, \eta, \mathrm{I}_{4 \mathrm{c}}, \lambda, \mathrm{t}\right):=\ldots \\
& \ldots:=\mathrm{A}_{1}\left(\mathrm{Y}_{1}, \sigma, \lambda_{1}, \lambda\right) \cdot \mathrm{I}_{1}\left(\tau_{1 \mathrm{a}}, \tau_{1 \mathrm{~b}}, \kappa, \mathrm{t}\right)+\mathrm{A}_{2}\left(\mathrm{Y}_{2}, \sigma, \lambda_{2}, \lambda\right) \cdot \mathrm{I}_{2}\left(\tau_{1 \mathrm{a}}, \tau_{1 \mathrm{~b}}, \tau_{2}, \kappa, \eta, \mathrm{t}\right)+\ldots \\
& \ldots+\mathrm{A}_{3} \cdot \mathrm{I}_{1}\left(\tau_{1 \mathrm{a}}, \tau_{1 \mathrm{~b}}, \kappa, \mathrm{t}\right)+\mathrm{A}_{4}\left(\mathrm{Y}_{4}, \sigma, \lambda_{4}, \lambda\right) \cdot \mathrm{I}_{4}\left(\mathrm{k}_{4 \mathrm{a}}, \mathrm{k}_{4 \mathrm{~b}}, \mathrm{I}_{4 \mathrm{c}}, \mathrm{t}\right)
\end{aligned}
$$




$$
\begin{aligned}
& \Delta \mathrm{OD}_{\text {conv }}\left(\mathrm{Y}_{1}, \mathrm{Y}_{2}, \mathrm{Y}_{3}, \mathrm{Y}_{4}, \sigma, \sigma_{\mathrm{IRF}}, \tau_{1 \mathrm{a}}, \tau_{1 \mathrm{~b}}, \tau_{2}, \mathrm{k}_{4 \mathrm{a}}, \mathrm{k}_{4 \mathrm{~b}}, \lambda_{1}, \lambda_{2}, \lambda_{3}, \lambda_{4}, \kappa, \eta, \mathrm{I}_{4 \mathrm{c}}, \lambda, \mathrm{t}\right):=\ldots \\
& \ldots:=\left(\int_{-\infty}^{\infty} \operatorname{IRF}\left(\sigma_{\operatorname{IRF}}, \mathrm{s}\right) \cdot \Delta \mathrm{OD}\left(\mathrm{Y}_{1}, \mathrm{Y}_{2}, \mathrm{Y}_{3}, \mathrm{Y}_{4}, \sigma, \tau_{1 \mathrm{a}}, \tau_{1 \mathrm{~b}}, \tau_{2}, \mathrm{k}_{4 \mathrm{a}}, \mathrm{k}_{4 \mathrm{~b}}, \lambda_{1}, \lambda_{2}, \lambda_{3}, \lambda_{4}, \kappa, \eta, \mathrm{I}_{4 \mathrm{c}}, \lambda, \mathrm{t}-\mathrm{s}\right) \mathrm{ds}\right)
\end{aligned}
$$

曰

Area 2: Initial/guessed values:

$\nabla$

$$
\begin{array}{lllll}
\mathrm{Y}_{1}:=-0.475 & \lambda_{1}:=442 \quad \tau_{1 \mathrm{a}}:=11.7 & \tau_{1 \mathrm{~b}}:=996 \quad \kappa:=0.88 \\
\mathrm{Y}_{2}:=-0.7 & \lambda_{2}:=483 \quad \tau_{2}:=0.36 \quad \eta:=1.569 \quad \mathrm{Y}_{3}:=-0.852 \quad \lambda_{3}:=512 \\
\mathrm{Y}_{4}:=2.039 & \mathrm{I}_{4 \mathrm{c}}:=0 \quad \lambda_{4}:=537 \quad \mathrm{k}_{4 \mathrm{a}}:=\frac{1}{0.125} \quad \mathrm{k}_{4 \mathrm{~b}}:=\frac{1}{0.117} \quad \sigma:=12.763 \quad \sigma_{\mathrm{IRF}}:=0.15
\end{array}
$$

$\theta$

Area 3: Importing data file and extracting experimental data to fit:

$\nabla$

$$
\text { data_file := }
$$

... Idata, dat

$$
\begin{aligned}
& \mathrm{N}_{\text {Delta_t }}:=\operatorname{rows}(\text { data_file })-1 \\
& \mathrm{~N}_{\text {wavelength }}:=\operatorname{rows}\left(\text { data_file }^{\mathrm{T}}\right)-1 \\
& \mathrm{~N}_{\text {Delta_t }}=\text { ・ } \\
& \mathrm{N}_{\text {wavelength }}=\text { • } \\
& \text { Delta_tmin }:=1 \\
& \text { wavelength }_{\min }:=1 \\
& \left(\text { data_file }{ }^{\langle 0\rangle}\right)_{\text {Delta_t }}=\mathbf{I} \\
& {\left[\left(\text { data_file }^{\mathrm{T}}\right)^{\langle 0\rangle}\right]_{\text {wavelength }} \text { min }_{\text {in }}=\mathbf{I}} \\
& \text { Delta_t max }:=1032 \\
& \text { wavelength }_{\max }:=356 \\
& \left(\text { data_file }{ }^{\langle 0\rangle}\right)_{\text {Delta_t }}{ }_{\max }=\mathbf{I} \\
& {\left[\left(\text { data_file }^{\mathrm{T}}\right)^{\langle 0\rangle}\right]_{\text {wavelength }_{\max }}=\mathbf{=}}
\end{aligned}
$$

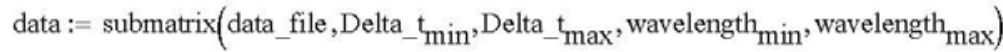


wavelength := submatrix(data_file, 0,0 , wavelength min $_{\text {, wavelength }}$ max $)^{\mathrm{T}}$

Delta_t:= submatrix(data_file,Delta_tmin,Delta_tmax $, 0,0)$

田

Area 4: Spectra, wavelength domain, multi-Gaussian, at t

$\nabla$

$\lambda:=430,431 . .620$

$\mathrm{N}:=81 \quad$ Delta_t $=\mathbf{N}$ fs

$\left(\text { data }^{\mathrm{T}}\right)^{\langle\mathrm{N}\rangle}$

$\Delta \mathrm{OD}_{\text {conv }}\left(\mathrm{Y}_{1}, \mathrm{Y}_{2}, \mathrm{Y}_{3}, \mathrm{Y}_{4}, \sigma, \sigma_{\mathrm{IRF}}, \tau_{1 \mathrm{a}}, \tau_{1 \mathrm{~b}}, \tau_{2}, \mathrm{k}_{4 \mathrm{a}}, \mathrm{k}_{4 \mathrm{~b}}, \lambda_{1}, \lambda_{2}, \lambda_{3}, \lambda_{4}, \kappa, \eta, \mathrm{I}_{4 \mathrm{c}}, \lambda, \frac{\text { Delta } \mathrm{t}_{\mathrm{N}}}{1000}\right)$

wavelength, $\lambda$

$\mathrm{N}:=120 \quad$ Delta_t $\mathrm{N}_{\mathrm{N}}=\mathbf{1}$ fs

$\left(\text { data }^{\mathrm{T}}\right)^{\langle\mathrm{N}\rangle}$

$\Delta \mathrm{OD}_{\text {conv }}\left(\mathrm{Y}_{1}, \mathrm{Y}_{2}, \mathrm{Y}_{3}, \mathrm{Y}_{4}, \sigma, \sigma_{\mathrm{IRF}}, \tau_{1 \mathrm{a}}, \tau_{1 \mathrm{~b}}, \tau_{2}, \mathrm{k}_{4 \mathrm{a}}, \mathrm{k}_{4 \mathrm{~b}}, \lambda_{1}, \lambda_{2}, \lambda_{3}, \lambda_{4}, \kappa, \eta, \mathrm{I}_{4 \mathrm{c}}, \lambda, \frac{\text { Delta_tw }}{1000}\right)$

wavelength, $\lambda$
$N:=377$
Delta_t $_{\mathrm{N}}=\mathbf{\mathrm { I }}$ fS

$\left(\text { data }^{\mathrm{T}}\right)^{\langle\mathrm{N}\rangle}$

$\Delta \mathrm{OD}_{\text {conv }}\left(\mathrm{Y}_{1}, \mathrm{Y}_{2}, \mathrm{Y}_{3}, \mathrm{Y}_{4}, \sigma, \sigma_{\mathrm{IRF}}, \tau_{1 \mathrm{a}}, \tau_{1 \mathrm{~b}}, \tau_{2}, \mathrm{k}_{4 \mathrm{a}}, \mathrm{k}_{4 \mathrm{~b}}, \lambda_{1}, \lambda_{2}, \lambda_{3}, \lambda_{4}, \kappa, \eta, \mathrm{I} 4 \mathrm{c}, \lambda, \frac{\text { Delta } \mathrm{t}_{\mathrm{N}}}{1000}\right)$

wavelength, $\lambda$

曰 
Area 5: TA spectra, time domain, at $\lambda$

$$
\Delta O D\left(Y_{1}, Y_{2}, Y_{3}, Y_{4}, \sigma, \tau_{1 a}, \tau_{1 b}, \tau_{2}, k_{4 a}, k_{4 b}, \lambda_{1}, \lambda_{2}, \lambda_{3}, \lambda_{4}, \kappa, \eta, I_{4 c}, 400,0\right)=\mathbf{I}
$$

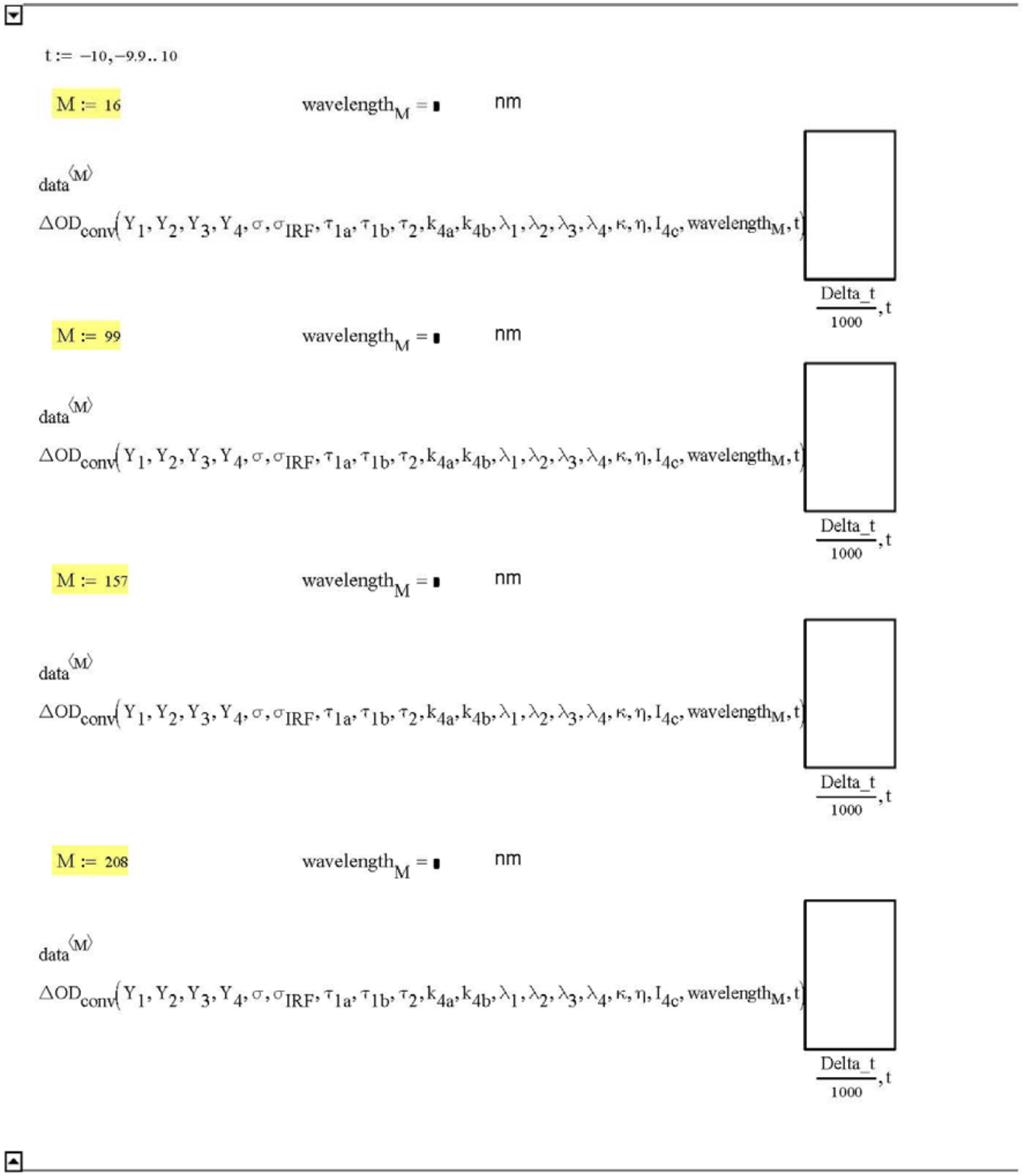




\section{Area 6: RMS}

stepsize $:=2$

$\nabla$

$\mathrm{i}:=45,45+$ stepsize.. 355

$\mathrm{j} 1:=16 \quad$ wavelength $_{\mathrm{j} 1}=$ ।

$\operatorname{RMSl}\left(\mathrm{Y}_{1}, \mathrm{Y}_{2}, \mathrm{Y}_{3}, \mathrm{Y}_{4}, \sigma, \sigma_{\mathrm{IRF}}, \tau_{1 \mathrm{a}}, \tau_{1 \mathrm{~b}}, \tau_{2}, \mathrm{k}_{4 \mathrm{a}}, \mathrm{k}_{4 \mathrm{~b}}, \lambda_{1}, \lambda_{2}, \lambda_{3}, \lambda_{4}, \kappa, \eta, \mathrm{I}_{4 \mathrm{c}}\right):=\ldots$

$$
\sum_{\mathrm{i}}\left(\Delta \mathrm{OD}_{\mathrm{conv}}\left(\mathrm{Y}_{1}, \mathrm{Y}_{2}, \mathrm{Y}_{3}, \ldots, \lambda_{2}, \lambda_{3}, \lambda_{4}, \kappa, \eta, \mathrm{I}_{4 \mathrm{c}}, \text { wavelength }_{\mathrm{j} 1}, \frac{\text { Delta }_{\mathrm{t}}}{1000}\right)-\text { data }_{\mathrm{i}, \mathrm{j} 1}\right)^{2}
$$

$\mathrm{j} 2:=99 \quad$ wavelength $_{\mathrm{j} 2}=\mathbf{I}$

$\operatorname{RMS} 2\left(\mathrm{Y}_{1}, \mathrm{Y}_{2}, \mathrm{Y}_{3}, \mathrm{Y}_{4}, \sigma, \sigma_{\mathrm{IRF}}, \tau_{1 \mathrm{a}}, \tau_{1 \mathrm{~b}}, \tau_{2}, \mathrm{k}_{4 \mathrm{a}}, \mathrm{k}_{4 \mathrm{~b}}, \lambda_{1}, \lambda_{2}, \lambda_{3}, \lambda_{4}, \kappa, \eta, \mathrm{I}_{4 \mathrm{c}}\right):=\ldots$

$$
\sum_{i}\left(\Delta O D_{\text {conv }}\left(Y_{1}, Y_{2}, Y_{3}, \ldots, \lambda_{2}, \lambda_{3}, \lambda_{4}, \kappa, \eta, I_{4 c}, \text { wavelength }_{j 2}, \frac{\text { Delta }_{\text {t }}}{1000}\right)-\text { data }_{1, j 2}\right)^{2}
$$

$\mathrm{j} 3:=157 \quad$ wavelength $_{\mathrm{j} 3}=\mathbf{I}$

$\operatorname{RMS} 3\left(\mathrm{Y}_{1}, \mathrm{Y}_{2}, \mathrm{Y}_{3}, \mathrm{Y}_{4}, \sigma, \sigma_{\mathrm{IRF}}, \tau_{1 \mathrm{a}}, \tau_{1 \mathrm{~b}}, \tau_{2}, \mathrm{k}_{4 \mathrm{a}}, \mathrm{k}_{4 \mathrm{~b}}, \lambda_{1}, \lambda_{2}, \lambda_{3}, \lambda_{4}, \kappa, \eta, \mathrm{I}_{4 \mathrm{c}}\right):=\ldots$

$$
\sum_{\mathrm{i}}\left(\Delta \mathrm{OD}_{\mathrm{conv}}\left(\mathrm{Y}_{1}, \mathrm{Y}_{2}, \mathrm{Y}_{3}, \ldots, \lambda_{2}, \lambda_{3}, \lambda_{4}, \kappa, \eta, \mathrm{I}_{4 \mathrm{c}}, \text { wavelength }_{\mathrm{j} 3}, \frac{\text { Delta }_{\mathrm{t}}}{1000}\right)-\text { data }_{\mathrm{i}, \mathrm{j} 3}\right)^{2}
$$

$\mathrm{j} 4:=208 \quad$ wavelength $_{\mathrm{j} 4}=\mathbf{I}$

$\operatorname{RMS} 4\left(\mathrm{Y}_{1}, \mathrm{Y}_{2}, \mathrm{Y}_{3}, \mathrm{Y}_{4}, \sigma, \sigma_{\mathrm{IRF}}, \tau_{1 \mathrm{a}}, \tau_{1 \mathrm{~b}}, \tau_{2}, \mathrm{k}_{4 \mathrm{a}}, \mathrm{k}_{4 \mathrm{~b}}, \lambda_{1}, \lambda_{2}, \lambda_{3}, \lambda_{4}, \kappa, \eta, \mathrm{I}_{4 \mathrm{c}}\right):=\ldots$

$$
\sum_{\mathrm{i}}\left(\Delta \mathrm{OD}_{\text {conv }}\left(\mathrm{Y}_{1}, \mathrm{Y}_{2}, \mathrm{Y}_{3}, \ldots, \lambda_{2}, \lambda_{3}, \lambda_{4}, \kappa, \eta, \mathrm{I}_{4 \mathrm{c}}, \text { wavelength }_{\mathrm{j} 4}, \frac{\text { Delta }_{\mathrm{t}}}{1000}\right)-\text { data }_{\mathrm{i}, \mathrm{j} 4}\right)^{2}
$$




$$
\begin{aligned}
& \operatorname{RMS}\left(\mathrm{Y}_{1}, \mathrm{Y}_{2}, \mathrm{Y}_{3}, \mathrm{Y}_{4}, \sigma, \sigma_{\mathrm{IRF}}, \tau_{1 \mathrm{a}}, \tau_{1 \mathrm{~b}}, \tau_{2}, \mathrm{k}_{4 \mathrm{a}}, \mathrm{k}_{4 \mathrm{~b}}, \lambda_{1}, \lambda_{2}, \lambda_{3}, \lambda_{4}, \kappa, \eta, \mathrm{I}_{4 \mathrm{c}}\right):=\ldots \\
& \operatorname{RMS} 1\left(\mathrm{Y}_{1}, \mathrm{Y}_{2}, \mathrm{Y}_{3}, \mathrm{Y}_{4}, \sigma, \sigma_{\mathrm{IRF}}, \tau_{1 \mathrm{a}}, \tau_{1 \mathrm{~b}}, \tau_{2}, \mathrm{k}_{4 \mathrm{a}}, \mathrm{k}_{4 \mathrm{~b}}, \lambda_{1}, \lambda_{2}, \lambda_{3}, \lambda_{4}, \kappa, \eta, \mathrm{I}_{4 \mathrm{c}}\right)+\ldots \\
& \operatorname{RMS} 2\left(\mathrm{Y}_{1}, \mathrm{Y}_{2}, \mathrm{Y}_{3}, \mathrm{Y}_{4}, \sigma, \sigma_{\mathrm{IRF}}, \tau_{1 \mathrm{a}}, \tau_{1 \mathrm{~b}}, \tau_{2}, \mathrm{k}_{4 \mathrm{a}}, \mathrm{k}_{4 \mathrm{~b}}, \lambda_{1}, \lambda_{2}, \lambda_{3}, \lambda_{4}, \kappa, \eta, \mathrm{I}_{4 \mathrm{c}}\right)+\ldots \\
& \operatorname{RMS} 3\left(\mathrm{Y}_{1}, \mathrm{Y}_{2}, \mathrm{Y}_{3}, \mathrm{Y}_{4}, \sigma, \sigma_{\mathrm{IRF}}, \tau_{1 \mathrm{a}}, \tau_{1 \mathrm{~b}}, \tau_{2}, \mathrm{k}_{4 \mathrm{a}}, \mathrm{k}_{4 \mathrm{~b}}, \lambda_{1}, \lambda_{2}, \lambda_{3}, \lambda_{4}, \kappa, \eta, \mathrm{I}_{4 \mathrm{c}}\right)+\ldots \\
& \operatorname{RMS} 4\left(\mathrm{Y}_{1}, \mathrm{Y}_{2}, \mathrm{Y}_{3}, \mathrm{Y}_{4}, \sigma, \sigma_{\mathrm{IRF}}, \tau_{1 \mathrm{a}}, \tau_{1 \mathrm{~b}}, \tau_{2}, \mathrm{k}_{4 \mathrm{a}}, \mathrm{k}_{4 \mathrm{~b}}, \lambda_{1}, \lambda_{2}, \lambda_{3}, \lambda_{4}, \kappa, \eta, \mathrm{I}_{4 \mathrm{c}}\right)
\end{aligned}
$$

$\vartheta$

$$
\operatorname{RMS}\left(\mathrm{Y}_{1}, \mathrm{Y}_{2}, \mathrm{Y}_{3}, \mathrm{Y}_{4}, \sigma, \sigma_{\mathrm{IRF}}, \tau_{1 \mathrm{a}}, \tau_{1 \mathrm{~b}}, \tau_{2}, \mathrm{k}_{4 \mathrm{a}}, \mathrm{k}_{4 \mathrm{~b}}, \lambda_{1}, \lambda_{2}, \lambda_{3}, \lambda_{4}, \kappa, \eta, \mathrm{I}_{4 \mathrm{c}}\right)=\mathbf{I}
$$

Area7 : global fitting

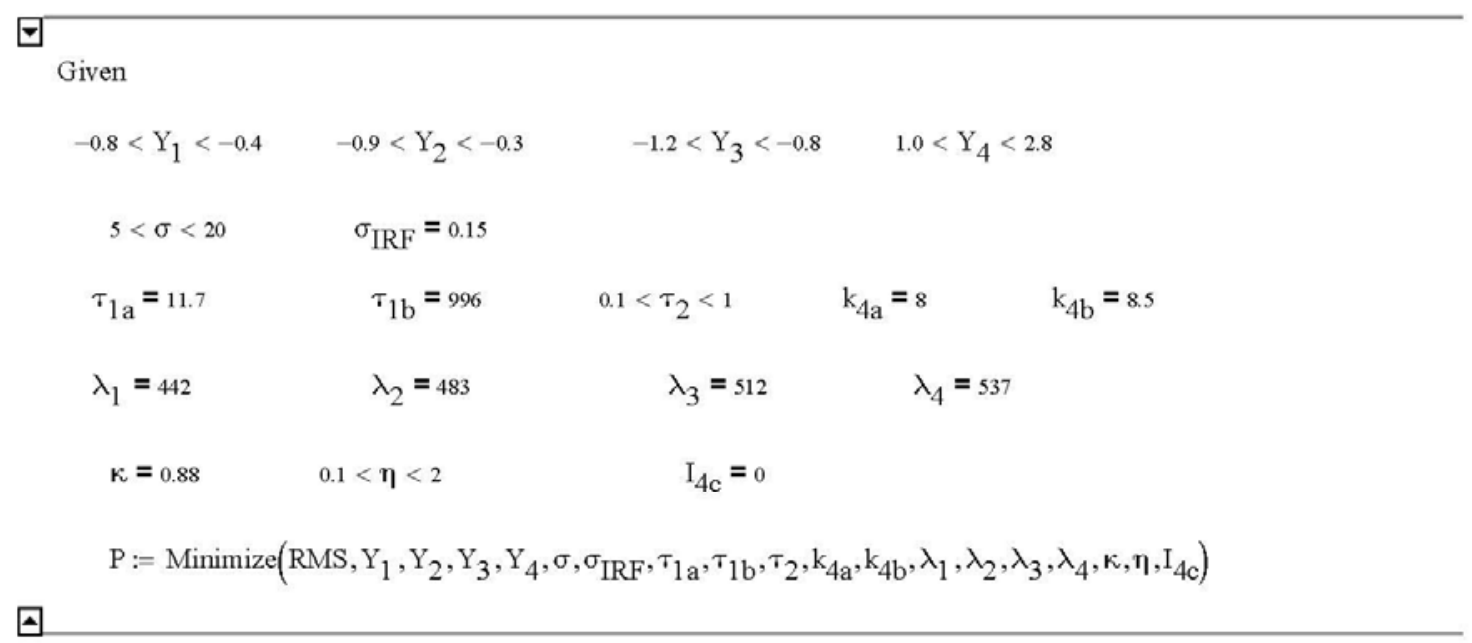

It takes about 10-20 minutes to finish the fitting!

$$
\mathrm{P}^{\mathrm{T}}=\mathbf{I}
$$$$
\operatorname{RMS}\left(\mathrm{P}_{0}, \mathrm{P}_{1}, \mathrm{P}_{2}, \mathrm{P}_{3}, \mathrm{P}_{4}, \mathrm{P}_{5}, \mathrm{P}_{6}, \mathrm{P}_{7}, \mathrm{P}_{8}, \mathrm{P}_{9}, \mathrm{P}_{10}, \mathrm{P}_{11}, \mathrm{P}_{12}, \mathrm{P}_{13}, \mathrm{P}_{14}, \mathrm{P}_{15}, \mathrm{P}_{16}, \mathrm{P}_{17}\right)=\text { | }
$$ 


\section{APPENDIX III LIST OF ABBREVIATIONS}

\begin{tabular}{|c|c|}
\hline BBO & $\beta$-barium borate \\
\hline BQ & Benzoquinone \\
\hline BRF & Birefringent filter \\
\hline CCD & Charge coupled device \\
\hline CPA & Chirped pulse amplification \\
\hline CRDS & Cavity ring-down spectroscopy \\
\hline CT & Charge transfer \\
\hline CTS & Charge transfer state \\
\hline CW & Continuous wave \\
\hline DCM & Dichloromethane \\
\hline DDA & Dodecylamine \\
\hline DDT & Dodecanethiol \\
\hline DFG & Difference frequency generation \\
\hline DOS & Density of states \\
\hline EMS & Emergency power supply \\
\hline ET & Electron transfer \\
\hline ETL & Electron transfer layer \\
\hline FRET & Fluorescence resonance energy transfer \\
\hline FROG & Frequency-resolved optical gating \\
\hline fs & Femtosecond \\
\hline FTO & Fluorine-doped tin oxide \\
\hline FWHM & Full width at half maximum \\
\hline GaAs & Gallium arsenide \\
\hline GRENOUILLE & $\begin{array}{l}\text { Grating-eliminated no-nonsense observation of ultrafast incident laser } \\
\text { light e-fields }\end{array}$ \\
\hline GVD & Group velocity dispersion \\
\hline GW & Gigawatt \\
\hline HDA & Hexadecylamine \\
\hline $\mathrm{HeNe}$ & Helium-neon \\
\hline HOMO & Highest occupied molecular orbital \\
\hline HT & Hole transfer \\
\hline HTL & Hole transfer layer \\
\hline
\end{tabular}




\begin{tabular}{|c|c|}
\hline IRF & Instrument response function \\
\hline KLM & Kerr-lens mode-locking \\
\hline LUMO & Lowest unoccupied molecular orbital \\
\hline MASER & Microwave amplification by stimulated emission of radiation \\
\hline MEG & Multiple exciton generation \\
\hline MO & Molecular orbital \\
\hline MRI & Magnetic resonance imaging \\
\hline MW & Megawatt \\
\hline NMR & Nuclear magnetic resonance \\
\hline NOPA & Nonlinear optical parametric amplification \\
\hline ns & Nanosecond \\
\hline OD & Optical Density \\
\hline OLED & Organic light emitting diode \\
\hline OPA & Optical parametric amplification \\
\hline OPG & Optical parametric generation \\
\hline OPO & Optical parametric oscillator \\
\hline PA & Photoabsorption \\
\hline PAGE & Polyacrylamide gel electrophoresis \\
\hline PB & Photobleaching \\
\hline PBS & Polarization beam splitter \\
\hline PCE & Power conversion efficiency \\
\hline PDTC & Phenyldithiocarbamate \\
\hline PL & Photoluminescence \\
\hline Py & Pyridine \\
\hline QDs & Quantum dots \\
\hline QDSC & Quantum-dot solar cell \\
\hline QLED & QDs-based light emitting diode \\
\hline RGA & Regenerative amplifier \\
\hline SCNCS & Semiconductor nanoclusters \\
\hline SErF & Single-mode Erbium doped fiber \\
\hline SERS & Surface enhanced raman spectroscopy \\
\hline SF & Self-focusing \\
\hline SFG & Sum frequency generation \\
\hline SG & Supercontinuum generation \\
\hline SHG & Second harmonic generation \\
\hline SPIDER & Spectral phase interferometry for direct electric-field reconstruction \\
\hline SPM & Self phase modulation \\
\hline $\mathrm{SRH}$ & Shockley-read-hall \\
\hline TA & Transient Absorption \\
\hline TAPPS & Transient absorption pump-probe spectroscopy \\
\hline TDPA & Tetradecylphosphonic acid \\
\hline
\end{tabular}




$\begin{array}{ll}\text { THG } & \text { Third harmonic generation } \\ \mathrm{TiO}_{2} & \text { Titanium dioxide } \\ \text { TOP } & \text { Trioctyl-phosphine } \\ \text { TOPA } & \text { Tetradecylphosphonic acid } \\ \text { TOPO } & \text { Trioctylphosphine oxide } \\ \text { TOPSe } & \text { Trioctylphosphine selenide } \\ \text { TW } & \text { Terawatts } \\ \text { WL } & \text { White-light } \\ \text { WP } & \text { Wave plate } \\ \text { X-PDTC } & \text { Para-substituted phenyldithiocarbamate derivative }\end{array}$




\section{APPENDIX IV GROUP VELOCITY DISPERSION CORRECTION}

When supercontinuum white light is used as a probe source for the pump-probe transient absorption spectroscopy, the transmitted white light is dispersed onto the array detector by a curved grating mirror. Due to this dispersion, photons with different wavelengths arrive at the detector at different times. In other words, the time-zero at each wavelength is shifted by an error value from the original zero position, which is set up by the positions of the pump and probe delay stages before the data acquisition starts. These time-zero errors need to be corrected before further data analyzing procedures.

First, to retrieve temporal domain traces from the original three-dimensional TA spectrum, twenty equally spaced wavelengths are selected to cover the full range of the spectral domain $(\lambda=435,445,455, \ldots \ldots 645,655 \mathrm{~nm})$. On each of these temporal domain traces, the time-zero is shifted from zero to $\Delta \mathbf{t}(\lambda)$, and the latter can be determined at the $\mathrm{x}-$ axis value whenever $\triangle \mathrm{OD}$ (y-axis) starts rising or decreasing due to the instrument response function. A series of $\Delta \mathbf{t}(\lambda)$ values are written down and plotted against $\lambda$ in Origin. The

plotted trace is fitted by a power function : $\Delta t(\lambda)=\Delta t_{0}+A\left|\lambda-\lambda_{0}\right|^{p}$, in order to determine the parameters which will be used in the GVD.exe program.

Where $\lambda_{0}$ is the reference wavelength chosen between $435-445 \mathrm{~nm} . \Delta \mathrm{t}_{0}$ is the value of $\Delta \mathbf{t}(\lambda)$ at wavelength $\lambda_{0}$. Once variables A and $\mathrm{p}$ are determined, the GVD correction can be performed by running the GVD.exe program. 
The GVD.exe program is obtained by complying a $\mathbf{C}$ source code file into an executable (.exe) file. Upon opening the program, the user will be asked to type in the value of $\mathrm{A}, \lambda_{0}, \mathrm{p}, \Delta \mathrm{t}_{0}$, in the right order, separated by space. The program also gives recommended values based on previous experimental data. Then the values typed in by the user are shown on the program dialog window for confirmation. Then the program asks the user to choose between linear interpolation and cubic interpolation. For the work present here, only the linear interpolation method was used. For wavelength calibration, "real wavelength" and "read wavelength" of CPA-fundamental need to be provided to the program. "Real wavelength" is always $775 \mathrm{~nm}$, and the experimental wavelength can be read from the wavelength domain spectra. The "read wavelength" usually ranges from $770-780 \mathrm{~nm}$, but sometimes is off by more than $40 \mathrm{~nm}$ due to improper calibration before the experiments.

The user is then asked to choose the number of points for the running average, if " 1 " is typed in, no running average will be performed, if "2" is typed in, one data point will be added for running average. The maximum value that can be typed in is "1024" where 1023 data points will be added. The GVD correction can be performed on one data file or multiple data files. All the data file(s) need to be put in the same folder as the GVD.exe. When only one file needs to be processed, the file name (ended with .txt extension) needs to be provided. When multiple files need to be processed, a batch file named in the form of XXXX.txt (maximum 32 characters) is created in the same folder. All the names (ended with ttxt extension) of the input files are listed in the batch file.txt. Once the name of the single file or the batch file is typed into the program, GVD correction will be initiated, and an (or a list of) output file(s) will be generated by the GVD.exe, and placed in the same folder.

C source code file: 


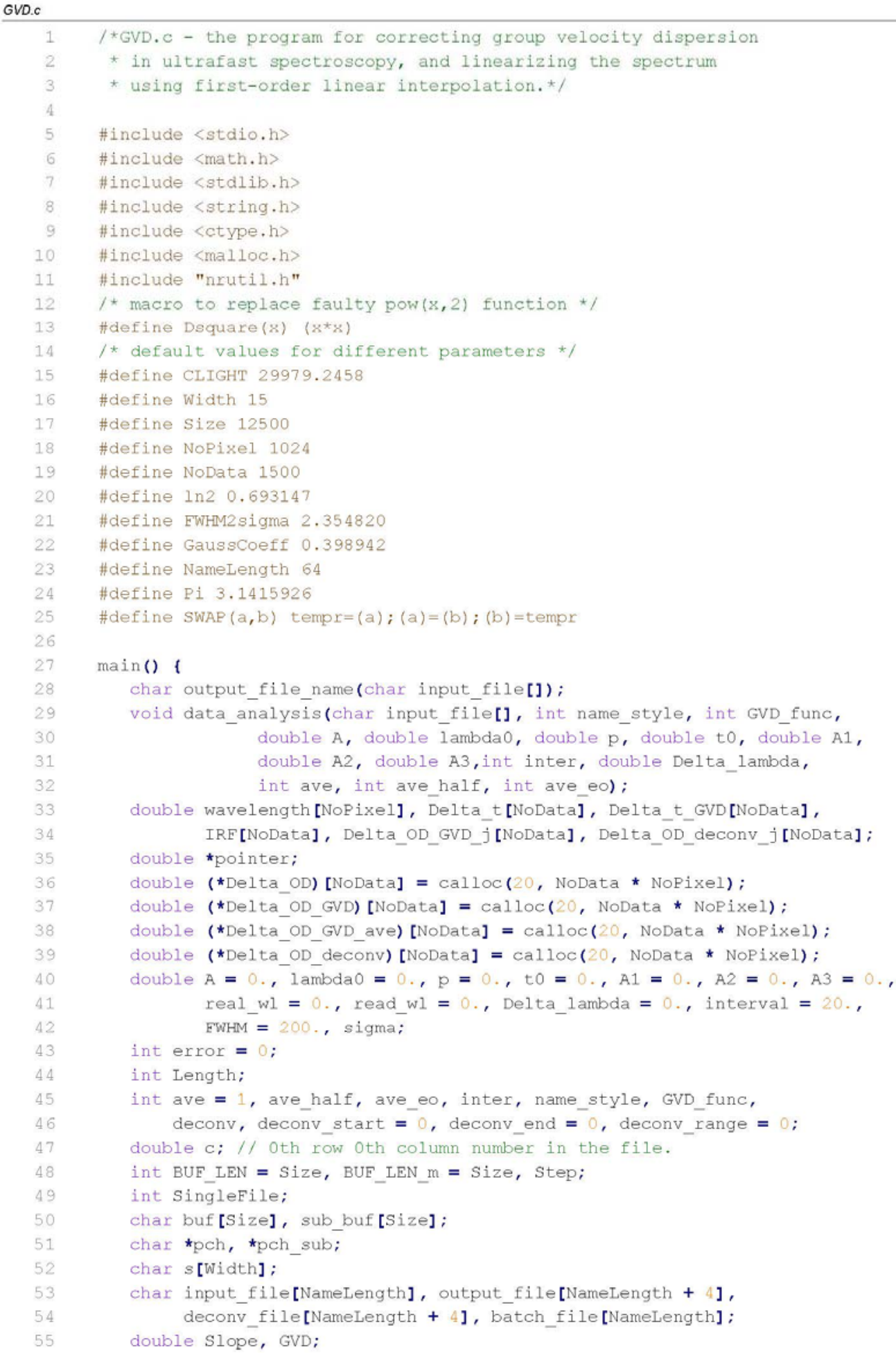

8.1 
FILE *ip, *op, *op deconv, *op IRE, *batch;

int i, j, $k, 1, \mathrm{~m}, \mathrm{n}, \mathrm{o}$;

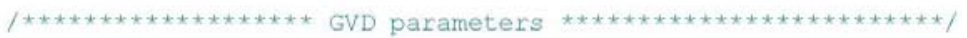

printf("8s \n", "Ultrafast Data Manipulation 5.0, " "Jinjun Liu, Louisvilie, 04/02/2014 (n");

printf("ss ln", "1. GVD Correction using either a power function or a " "third-order polynomial. At the moment, linear interpolation "

"is used. Cubic spline will be added in the future;");

printf("8s \n", "2. Calibration of time zero;");

printf("8s $\backslash \mathrm{n} "$, "3. Calibration of wavelength;");

printf("s) $\backslash n ", " 4$. Running average in wavelength domain, " "i.e., averaging of TA data point over different wavelengths " "with the same time delay;");

printf("os $\backslash \mathrm{n} "$, "5. Deconvolution by instrument response " "function (IRF) if requested.");

printf("8s $\backslash \mathrm{n} "$, "User can choose to carry out the data manipulation " "on individual files or multiple files after reading the file" "names listed in a batch file. (n");

do \{

printf("\&s $\backslash n$ ", "Please choose the GVD function: " "Type in '1' for a power function; " "'2" for a third-order polynomial.");

scanf_s("si", \&GVD_func);

if (GVD_func $==1$ ) \{ printf("ss $\backslash \mathrm{n} "$, "Power Function. In"); error $=0$;

) else

if (GVD_func $==2$ ) \{ printf("ss \n", "Third-Order Polynomial. \n"); error $=0$;

\} else \{ printf("No meaning for 'of' in GVD function. \n", inter); error $=1$;

\}

\} while (error $==1$ );

if (GVD func $==1$ ) \{ printf("ss $\backslash n "$, "GVD Eormula:"); printf("ss $\backslash n^{\prime}$, "Delta $t=A^{*}($ lambda-lambda0)^p+tO"); printf("ss $\backslash n "$, "t in $f s$ and lambda in $n m \backslash n ")$; 


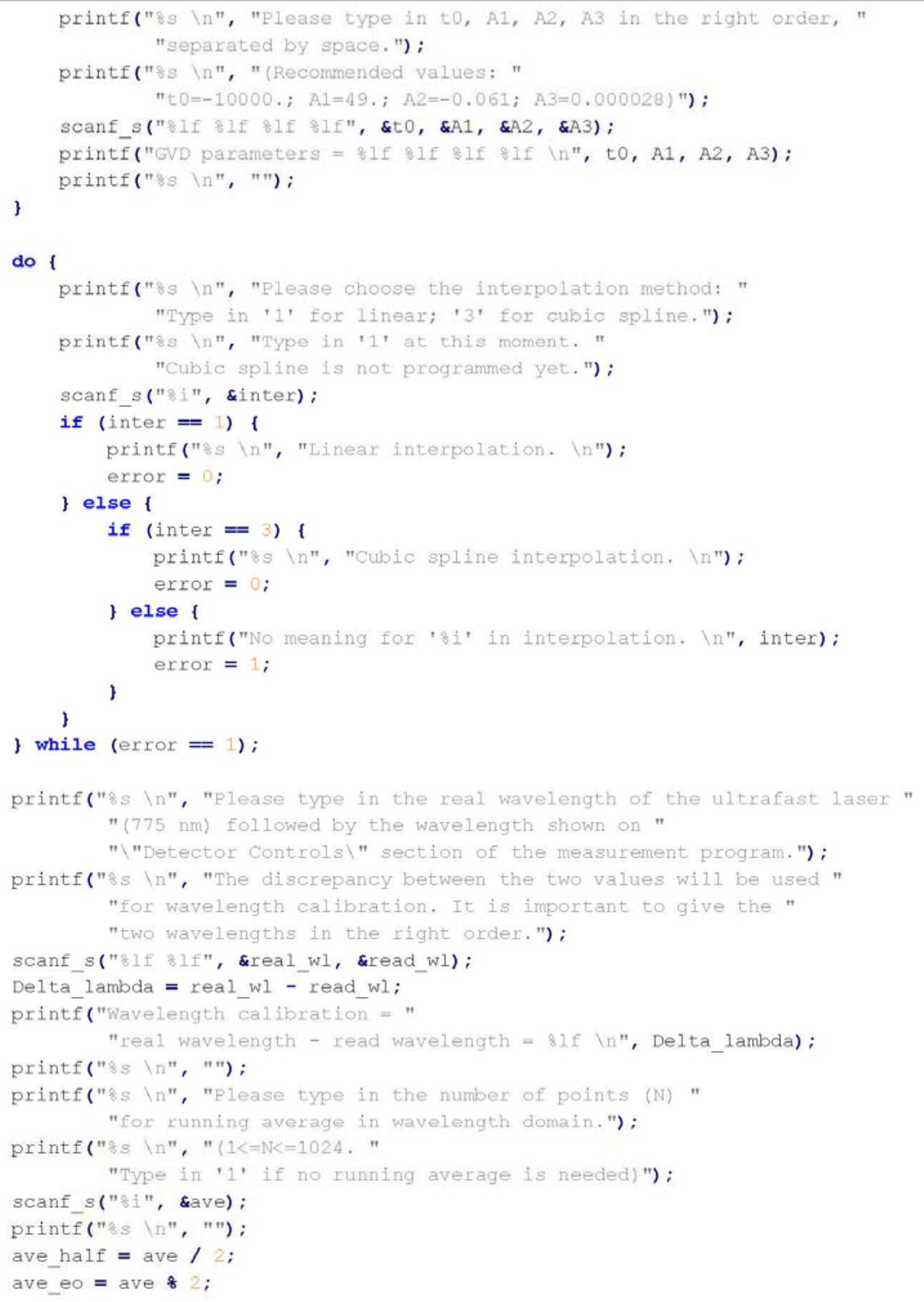




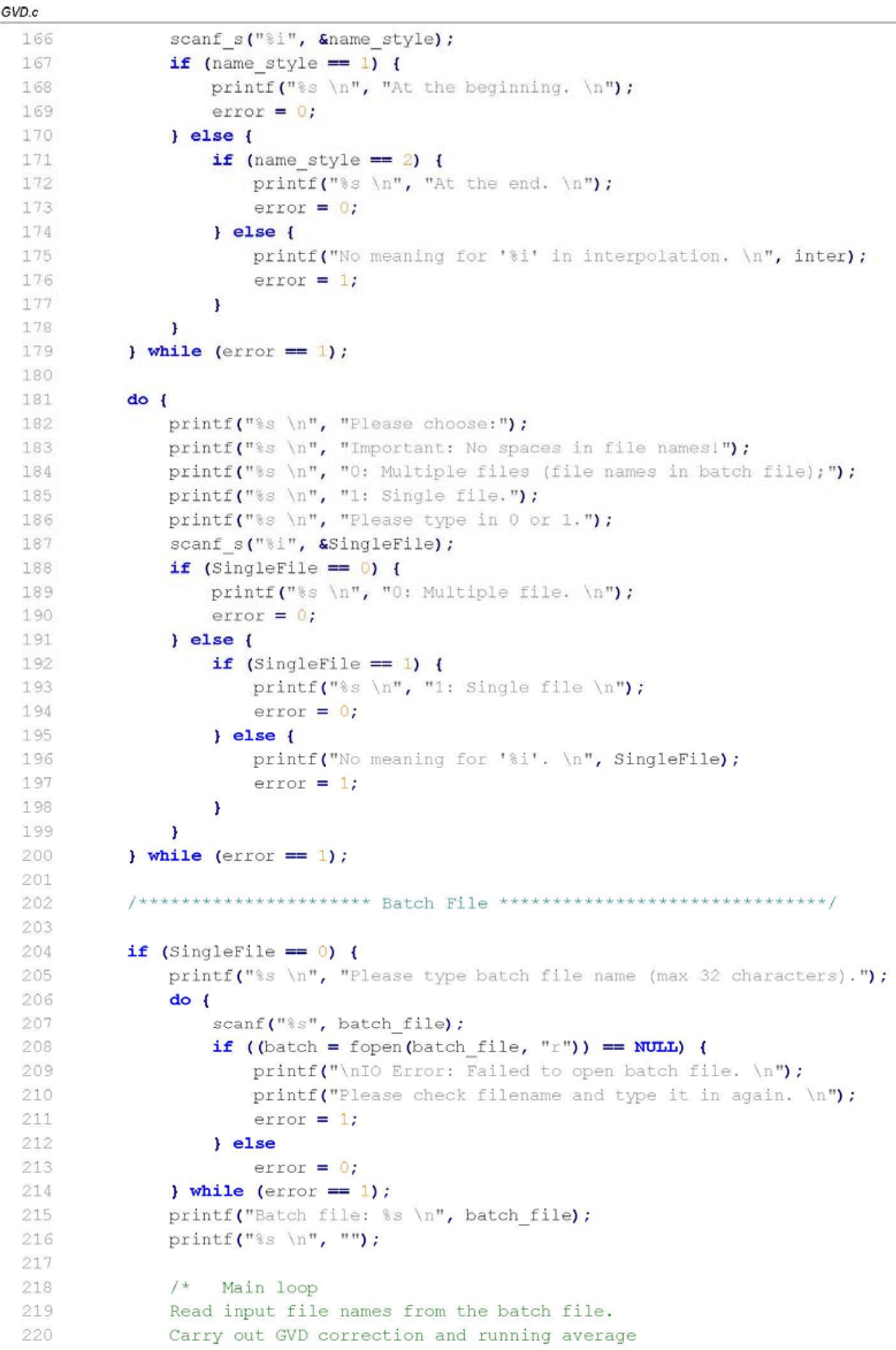




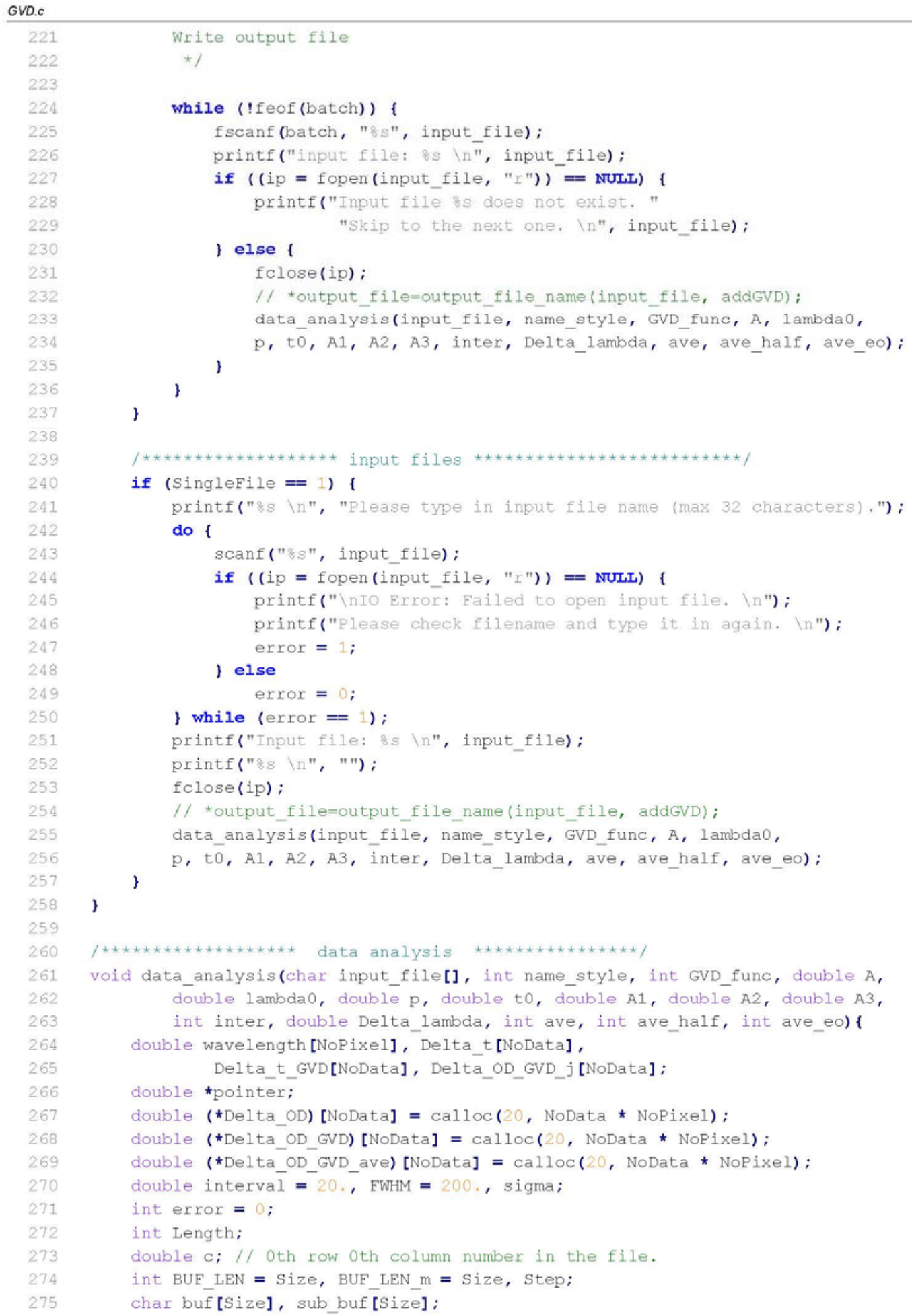

Write output file

$\star /$

while (!feof (batch)) \{

fscanf (batch, "os", input_file) ;

printf("input file: os $\backslash \mathrm{n} "$, input_file);

if $((i p=$ fopen (input_file, $" I "))==$ NULI) \{

printf("Input file os does not exist. "

"Skip to the next one. In", input_file);

\} else \{

fclose(ip) ;

// *output_file=output_file name (input_file, addGVD); data_analysis(input_file, name_style, GVD_func, A, lambdao,

\} p, to, A.1, A. , A3, inter, Delta_lambda, ave, ave_half, ave_eo);

\}

\}

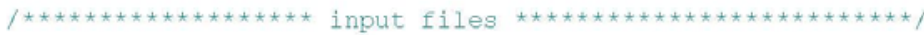

if (Singlefile == 1) \{

printf("8s \n", "please type in input file name (max 32 characters).");

do \{

scanf ("ss", input_file);

if $((i p=$ fopen (input_file, $" r "))==$ NULL) \{

printf("\nIO Error: Failed to open input file. In");

printf("Please check filename and type it in again. (n");

error $=1$;

\} else

error $=0$;

) while (error $==1$ );

printf("Input file: of $\backslash \mathrm{n}$ ", input_file);

printf("8s $\backslash n ", " n)$;

fclose(ip) ;

// *output_file=output_file_name(input_file, addGVD) ;

data_analysis(input_file, name_style, GVD_func, A, lambdao,

p, to, A1, A.2, A3, inter, Delta_lambda, ave, ave_half, ave_eo);

\} \}

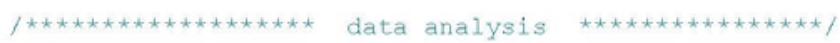

void data_analysis(char input_file[], int name_style, int GVD_func, double A, double lambda0, double p, double to, double A1, double A.2, double A3,

int inter, double Delta_lambda, int ave, int ave_half, int ave_eo) double wavelength [NoPixel], Delta_t[NoData], Delta_t_GVD[NoData], Delta_OD_GVD_j[NoData] ;

double *pointer;

double (*Delta OD) [NoData] $=\operatorname{calloc}(20$, NoData * NoPixel);

double (*Delta_OD_GVD) [NoData] $=\operatorname{calloc}(20$, NoData * NoPixel);

double (*Delta_OD_GVD_ave) [NoData] = calloc(20, NoData * NoPixel);

double interval $=20 .$, FWHM $=200 .$, sigma;

int error $=0$;

int Length;

double c; // Oth row oth column number in the file.

int BUF_LEN = Size, BUF_LEN_m = Size, Step;

char buf [Size], sub buf [Size] ; 


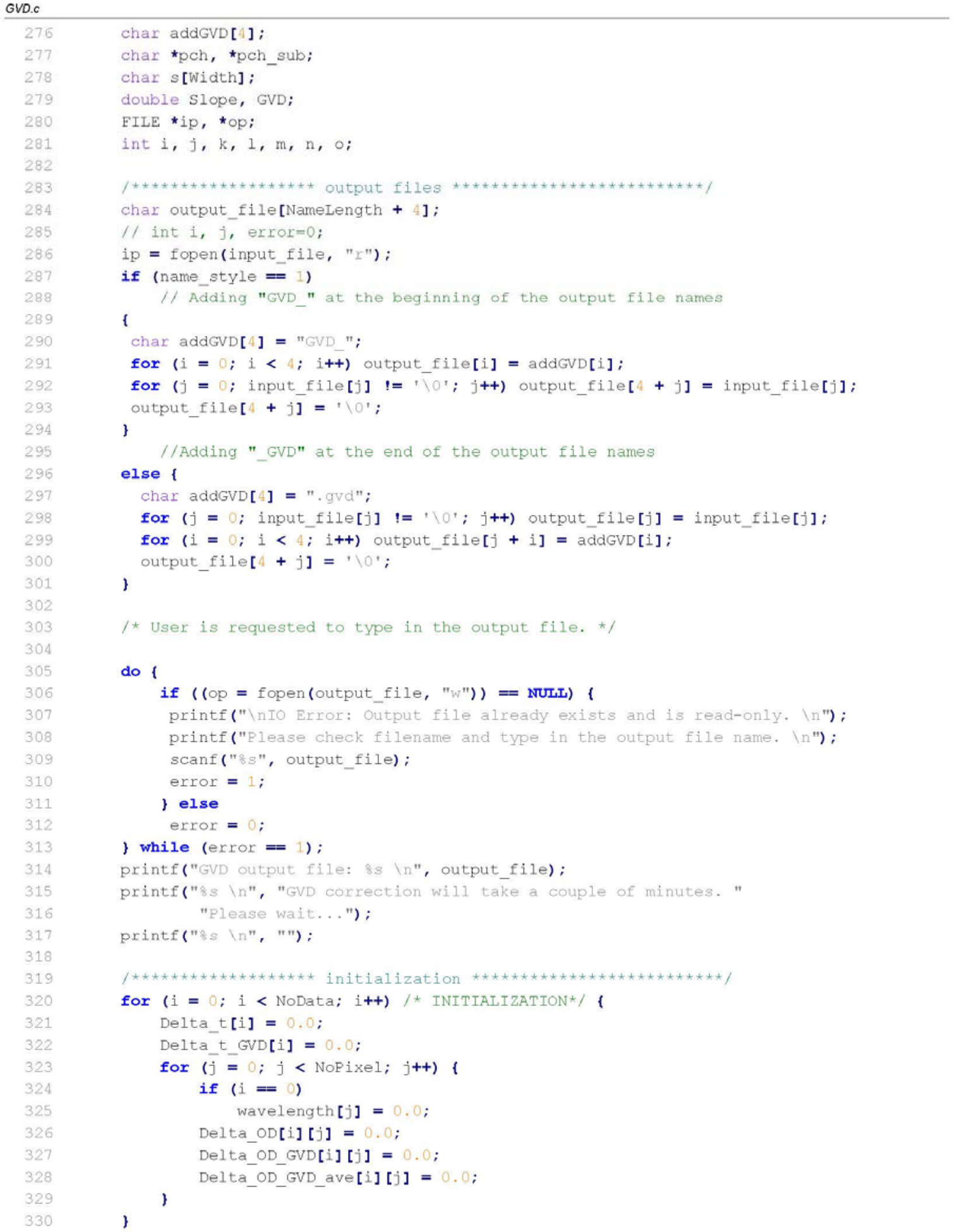




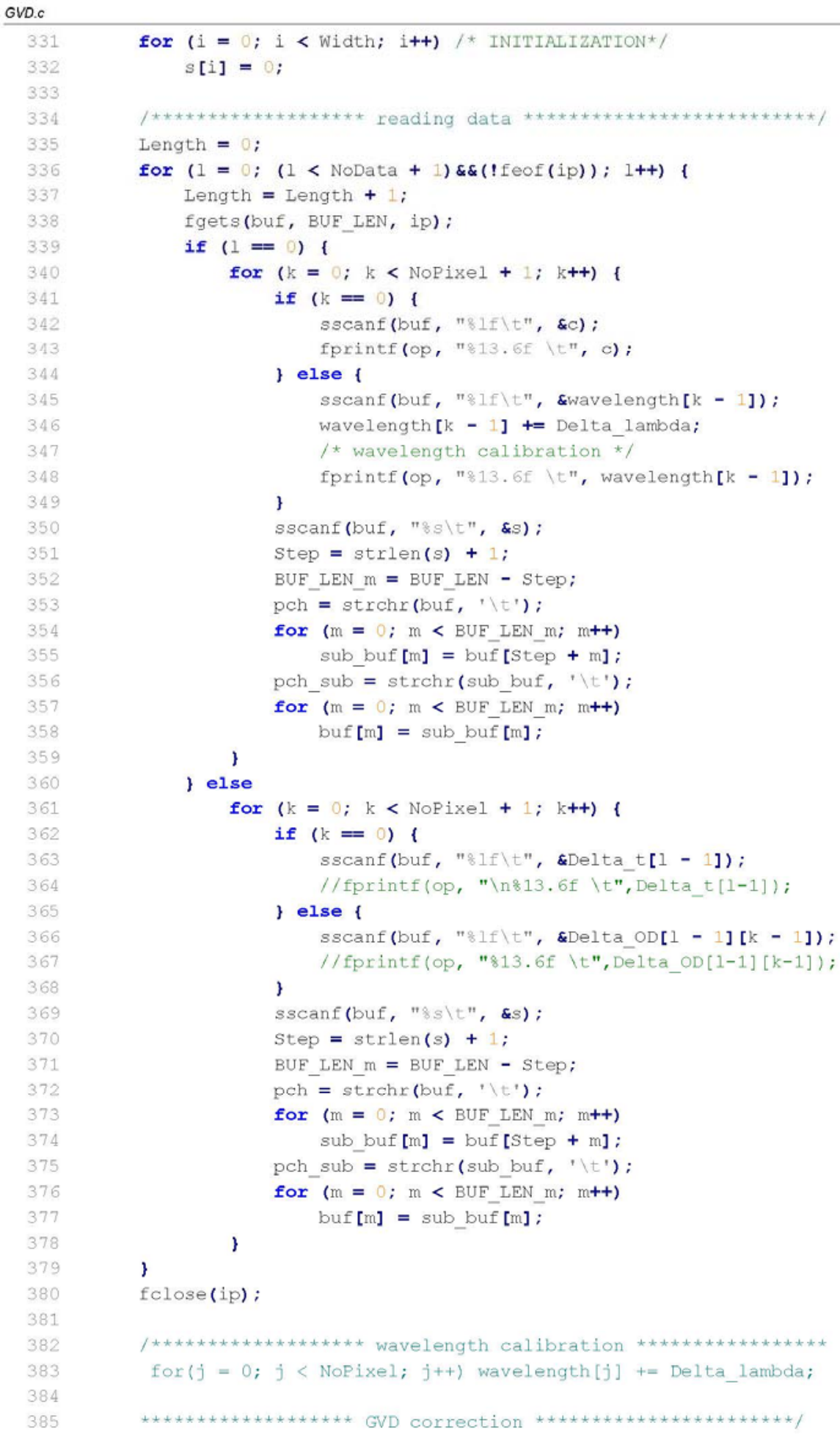




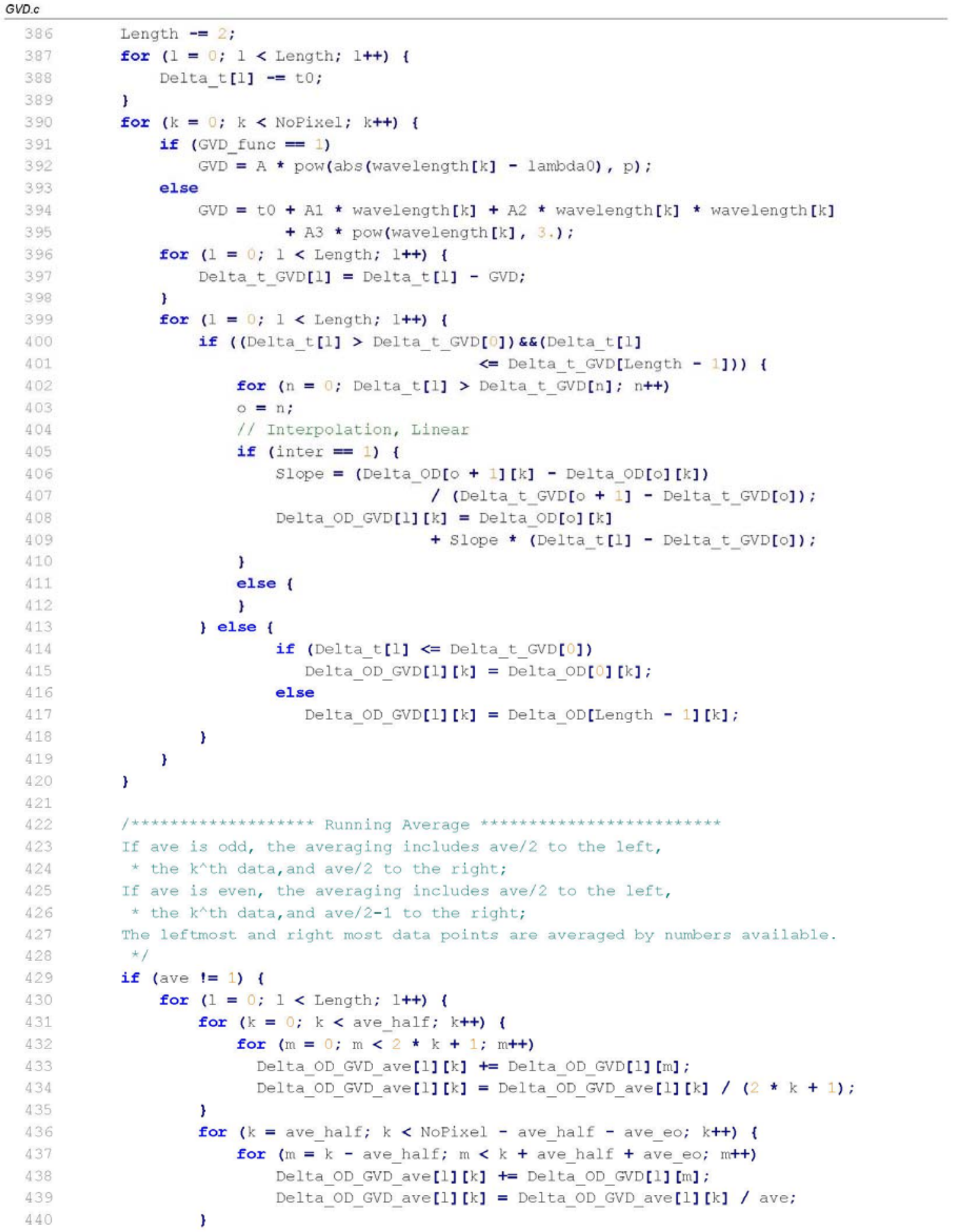




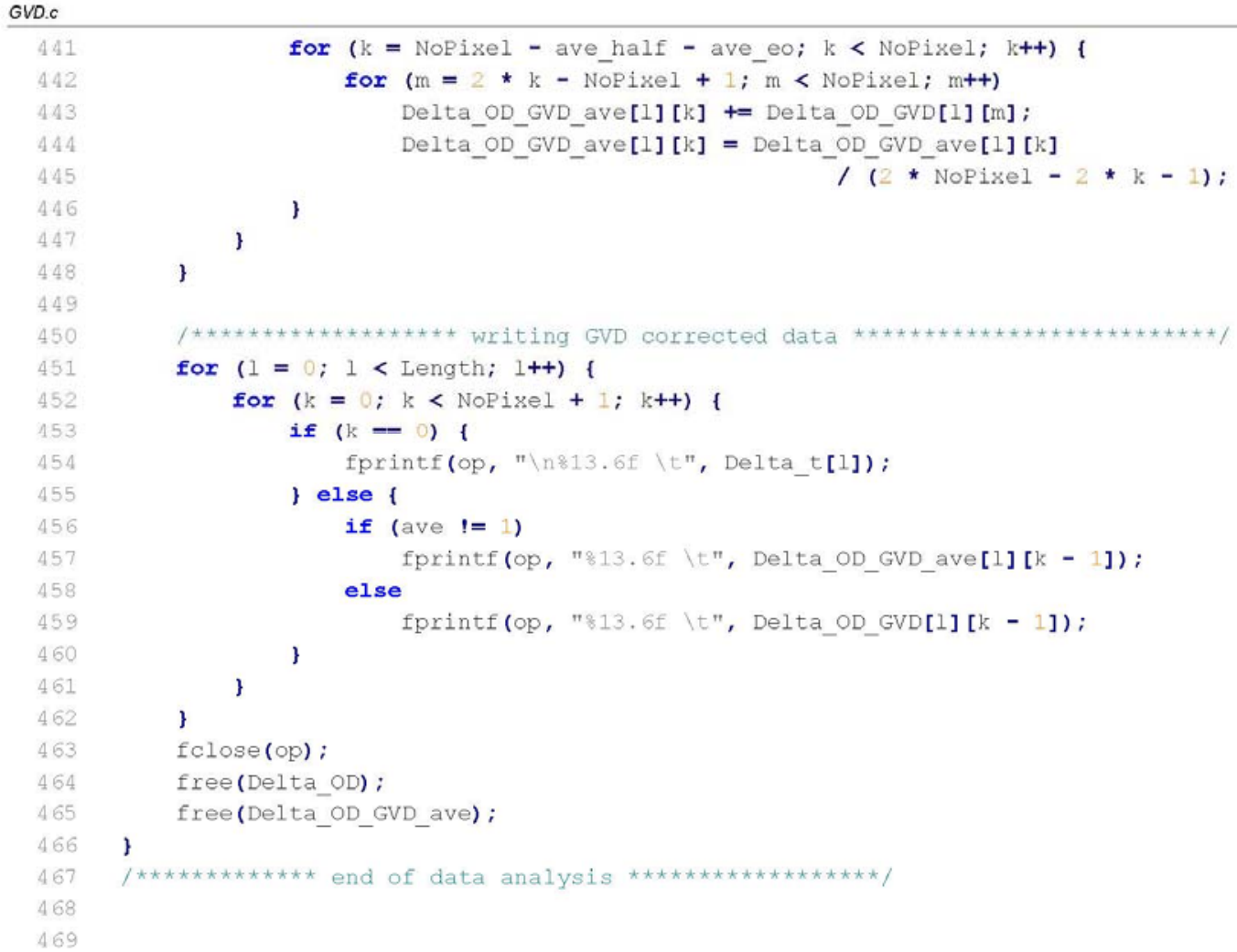




\title{
CURRICULUM VITAE
}

\author{
Yizhou Xie \\ (502) 471-6846 \\ y0xie003@1ouisville.edu
}

\section{OBJECTIVE}

R\&D position in the field of laser spectroscopy or instrumental analysis.

\section{PROFESSIONAL EXPERIENCES}

- Profound skills in the field of laser spectroscopy including fluorescence spectroscopy, cavity ring-down absorption spectroscopy, and ultrafast transient absorption spectroscopy.

- Hands-on experience with continuous-wave (cw), nanosecond, and femtosecond laser systems.

- Deep understanding of photovoltaic devices, nonlinear optics, laser technology, optoelectronics, data acquisition and processing.

- Years of experience in biochemistry and enzymology. Handling scientific balance, measuring pipette, volumetric flask and $\mathrm{pH}$ meter on a daily basis.

- Rich experience in gene expression, enzyme mutation, protein digestion, enzyme 3D modeling, and gel imaging. Proficient at instrumental analysis knowledge including HPLC, MALDI-TOF, UV-Vis and IR spectroscopy.

\section{EDUCATION}

Ph.D. in Chemistry,

University of Louisville, Louisville, KY, USA. May 2017

Mater of Science in Chemistry,

University of Louisville, Louisville, KY, USA. Dec 2012

Bachelor of Science in Chemistry,

Shandong University, Jinan, China. May 2007 


\section{WORK EXPERIENCE}

Research Associate

July 2016 - May 2017

Research Laboratory of High Resolution Laser Spectroscopy,

Department of Chemistry, University of Louisville, Louisville, KY, USA

- Studied cavity ring-down spectroscopy (CRDS) using cw single-frequency diodepumped Ti:Sapphire ring laser. Wrote LabView program for laser frequency control and data acquisition.

Part-time Faculty

Aug 2015 - Dec 2016

Department of Chemistry, Eastern Kentucky University, Richmond, KY, USA

- Lectured on General Chemistry and Organic Chemistry Laboratory.

Research Associate

Jan 2013 - May 2015

\section{Conn Center for Renewable Energy Research,}

University of Louisville, Louisville, KY

- Studied the energy level alignments and charge transfer dynamics of ligandpassivated CdSe quantum dots and nanoclusters using transient absorption pumpprobe spectroscopy (TAPPS). Built a theoretical model for the ultrafast (subpicosecond scale) kinetics in photovoltaic devices, and accurate determination of the time constants in photo-induced processes.

Research Assistant

April 2008 - Dec 2012

Department of Chemistry, University of Louisville, Louisville, KY, USA

- Developed an analytical method of HPLC-based assay. Preformed routine maintenance tasks for Agilent Series 1100 HPLC, Beckman system GOLD HPLC, and nanoITC.

- Investigated enzyme mechanism for Pseudouridine Synthases. Obtained information from trypsin-digested peptides and RNA nucleotides by using HPLC spectroscopy and mass spectroscopy fragmentation method. Worked with protein and DNA imaging using polyacrylamide gel (PAGE) and agarose gel.

Teaching Assistant Aug 2010 - May 2015

Department of Chemistry, University of Louisville, Louisville, KY, USA

- Lectured on Intro Chemistry, and Analytical Chemistry Laboratory. In charge of the lab preparation for Physical Chemistry Laboratory including electronic instrument repairs, chemical ordering, and laser optical alignments. Built a complete lab inventory and labeling system. 
- Determined the structure of nanoparticle micelle using X-ray diffraction. Built a phase diagram for lyotropic liquid crystal using scanning electron microscopy, microwave synthesis reactor, and circular dichroism.

\section{KEY SKILLS}

Lasers and Optics: cw Ti:Sapphire ring laser (Coherent), Nd:YAG laser (SpectraPhysics), pulsed dye laser (Sirah), femtosecond fiber laser and amplifier (Clark MXR), frequency up-conversion (SHG, THG), optical parametric amplifier (OPA).

Optical Spectroscopy: FTIR, fluorescence spectroscopy, cavity ring-down absorption spectroscopy, ultrafast transient absorption spectroscopy.

Molecular Biology: DNA transformation, protein expression/purification/digestion, enzyme activity assay, metabolites analyzing.

Bio-Laboratory Technique: working in the cold room $\left(4^{\circ} \mathrm{C}\right)$, handling $0.5 \mathrm{uL}-20 \mathrm{~mL}$ pipettes, making up to $20 \mathrm{~L}$ E.coli cell cultures, autoclaving, SDS/Urea-PAGE gel imaging, biuret assay.

Analytical Technique: HPLC, FPLC, MALDI-TOF, UV-Vis and IR spectroscopy.

Electronics: digital oscilloscopes, lock-in amplifiers, gated integrators (boxcars), pulse and delay generators, wave function generator, data acquisition cards.

Software: MathCAD, Origin, Adobe Illustrator, 3D MAX, Java, OnShape, Labview.

\section{SELECTED PUBLICATIONS}

- Yizhou Xie, Bill Pandit, Meghan B. Teunis, Rajesh Sardar, Jinjun Liu. Photoinduced Charge Transfer in Ligand-Passivated Nanoclusters: a Comparative Study. (in preparation)

- Yizhou Xie, Meghan B. Teunis, Bill Pandit, Rajesh Sardar, Jinjun Liu. Molecule-like CdSe Nanoclusters Passivated with Strongly Interacting Ligands: Energy Level Alignment and Photoinduced Ultrafast Charge Transfer Processes. Journal of Physical Chemistry C 2015, 119(5), p2813

- McDonald M.K., Miracco E.J., Chen J., Xie Y., Mueller E.G. The handling of the mechanistic probe 5-fluorouridine by the pseudouridine synthase TruA and its consistency pseudouridine synthases TruB and RluA. Biochemistry 2011, 50(3), p426 
- Zhang G., Chen X., Xie Y., Zhao Y., Qiu H. Lyotropic liquid crystalline phases in a ternary system of 1-hexadecyl-3-methylimidazolium chloride/1-decanol/water. $J$. Colloid Interface Sci 2007, 315(2), p601

- Jing B., Chen X., Wang X., Yang C., Xie Y. and Qiu, H. Self-assembly vesicles made from a cyclodextrinsupramolecular complex. Chem. Eur. J. 2007, 13, p9137

\section{SELECTED PRESENTATIONS}

- Yizhou Xie, Meghan B. Teunis, Bill Pandit, Rajesh Sardar, and Jinjun Liu. CdSe Semiconductor Nanoclusters Passivated with Strongly Interacting PDTC Ligands. 2015 RE3 workshop.

- Yizhou Xie and Eugene G. Mueller. Interesting Properties of Pus10, a Pseudouridine Synthase of Eukaryotes and Archaea. 2012 Rustbelt RNA Meeting.

\section{LANGUAGES}

Chinese: native speaker;

English: fluent.

\section{REFERENCE}

Dr. Jinjun Liu,

Associate Professor, Department of Chemistry and Conn Center for Renewable Energy Research, University of Louisville.

Phone: 502-852-1223 Email: j.liu@louisville.edu

Dr. Rajesh Sardar, Associate Professor, Department of Chemistry and Chemical Biology, Indiana University-Purdue University Indianapolis (IUPUI).

Phone: 317-278-2511 Email: rsardar@iupui.edu

Dr. Donghui Quan, Assistant Professor/Graduate Program Coordinator, Department of Chemistry, Eastern Kentucky University.

Phone: 859-622-7461 Email: donghui.quan@eku.edu 